\title{
SSP/SSTS - EFP Joint Annual Conference 2021
}

Fully digital, 17-18 June 2021 


\section{Pulmonary Rehabilitation, Patient Education and Palliative Care}

\section{P01 \\ Evaluation of the Implementation of the "Living Well with COPD" Self-Management Program in Switzerland}

\author{
A. Frei ', A. Strassmann ${ }^{1}$, M. Guler ${ }^{2}$, T. Carron ${ }^{3}$, C. Steurer-Stey' ${ }^{1}$, \\ K. Dalla Lana' ${ }^{1}$ P. Giroud ${ }^{2}$, I. Peytremann-Bridevaux ${ }^{3}$, M. Puhan ${ }^{1}$ \\ ${ }^{1}$ Epidemiology, Biostatistics and Prevention Institute, University \\ of Zurich, Zürich, ${ }^{2}$ Lungenliga Schweiz, Bern, ${ }^{3}$ Centre for Primary \\ Care and Public Health (Unisanté), University of Lausanne, \\ Lausanne, Switzerland
}

Introduction: The aim of this mixed-methods study was to evaluate the implementation of the "Living well with COPD" selfmanagement program, a nation-wide effort by the Swiss Lung Associations and the Swiss society of pulmonary medicine.

Methods: For the implementation evaluation we used qualitative (interviews, focus groups) and quantitative (questionnaires, documentation analysis) methods to assess indicators of the outcomes reach, dose, fidelity, acceptability and appropriateness of the program. To evaluate the effectiveness, we assessed disease specific quality of life (Chronic Respiratory Questionnaire CRQ), symptoms, exercise capacity, health care utilisation and health behaviour at baseline and at the end of the program (after 12 months).

Results: Seven Cantonal Lung Associations implemented the program into their services according to plan, conducted it 13 times during 1 year and included in total 122 COPD patients (mean age 69 years, $48 \%$ female). The patients' attendance rate and the coaches' fidelity to the protocol were high (81\% and $94 \%$, respectively). Overall, acceptance and satisfaction of all involved persons (patients, coaches, responsible persons from the Lung
Associations) was very high; they particularly acknowledged the meaningfulness of the program. Challenges were sustainable funding, integration of the coaches' additional workload and uncertainties regarding roles and responsibilities. After 14 month, the patients did not just avoid a decline in health status but statistically significantly improved in 3 of 4 CRQ subscales (with 0.2-0.4 units) and in the 1-minute sit-to-stand test (23.9 vs. 27.1 repetitions).

Conclusion: The "Living well with COPD" program was successfully implemented despite a tight schedule and showed a remarkably positive impact of the COPD patients' health status. The insights of this study will support the broader multiplication of the program throughout Switzerland and also serves the international community since it is one of the first nation-wide implementations beyond study settings.

Disclosure: Nothing to disclose

\section{P02 \\ Effects of a Comprehensive Pulmonary Rehabilitation in Severe Post-COVID-19 Patients}

M. Spielmanns ${ }^{1,2}$, A.-M. Pekacka-Egli ${ }^{1}$, S. Schoendorf ${ }^{1}$,
W. Windisch ${ }^{3}$, M. Hermann

${ }^{1}$ Pulmonary Medicine and Sleep Medicine Center, Zurich RehaCenter Klinik Wald, Wald, Switzerland, ${ }^{2}$ Department for Pulmonary Medicine, Faculty of Health, University WittenHerdecke, Witten, ${ }^{3}$ Department of Pneumology, Cologne Merheim Hospital, Kliniken der Stadt Koeln GmbH, windischw@ kliniken-koeln. de, Köln, Germany, ${ }^{4}$ Department of Cardiology, University Heart Center Zurich, University Hospital Zurich,

Zurich, Switzerland

Introduction: Severe COVID-19 infection often leads to impairments requiring pulmonary rehabilitation (PR) following the acute phase. Little is known about the efficacy of PR in these patients. We therefore compared Post-COVID-19 patients (PG) referred to PR patients with other lung diseases (LG). 
Methods: 99 PG were admitted to PR. In a prospective design the results of PG were collected and compared to the results of LG of 2019 ( $n=419)$ according to Functional Independence Measurement (FIM), Cumulative Illness Rating Scale (CIRS), 6-minute walktest (6-MWT), duration of PR, and Feeling Thermometer(FT).

Results: According to age, sex and CIRS both groups showed no significant differences. The improvements in the 6-MWT in the pre to post comparison were on average 180 (101) meter for PG and $102(89)$ meter for LG $(\mathrm{p}<0.001)$. FT showed a significant enhancement for PG of 21 (14) points and for LG of 17 (16) points $(p<0.039)$ while FIM significantly increased by $11(10)$ points in PG and 7 (8) points in LG $(\mathrm{p}<0.001)$.

Conclusions: Comprehensive PR in PG is very effective according to the results in FIM, 6-MWT and FT. Therefore, we recommend PR following severe Post-COVID-19 infections.

Disclosure: Nothing to disclose

\section{P03 \\ Recovery in Patients with SARS-CoV-2 Associated Respiratory Failure}

\author{
D. Kohlbrenner $^{1,2}$, M. Kuhn ${ }^{1,2}$, M. Stüssi-Helbling ${ }^{3}$, M. Spielmanns ${ }^{4}$, \\ Y. Nordmann ${ }^{5}$, C. F. Clarenbach ${ }^{1}$ \\ ${ }^{1}$ Department of Pulmonology, University Hospital Zurich, \\ ${ }^{2}$ Faculty of Medicine, University of Zurich, ${ }^{3}$ Clinic of Internal \\ Medicine, Department of Internal Medicine, Triemli Hospital, \\ Zurich, ${ }^{4}$ Department of Pulmonary Rehabilitation, Zürcher \\ Rehazentren Klinik Wald, Wald, ${ }^{5}$ docdok Health, Basel, \\ Switzerland
}

Introduction: Severe acute respiratory syndrome coronavirus 2 (SARS-CoV-2) is the cause of the current coronavirus disease (COVID-19) pandemic and can lead to respiratory failure requiring oxygen therapy. Data on symptom burden and health-related quality-of-life (HrQoL) during recovery from COVID-19 is scarce. Innovative mobile health equipment allows real-time monitoring after hospital discharge, and minimizes infection risks. We observed the post-hospital recovery via a smartphone application after a SARS-CoV-2 infection requiring oxygen therapy.

Methods: We conducted a longitudinal observational study with daily symptom burden, and weekly HrQoL and anxiety/ depression monitoring by smartphone-based questionnaires over 3 months. Symptomatic participants were included after hospital discharge. We used descriptive statistics to report longitudinal courses.

Results: We included 50 participants with a median age of 60 $(49,69)$ years. Median hospitalisation length was $10(6,16)$ days, with $16 \%$ admittance to intensive care units (ICU). Symptom burden in the general sample was considerable after hospitalization, symptoms resolved below the critical threshold 45 days after discharge. Participants admitted to ICU reported a similar symptom burden after discharge. However, their recovery was prolonged and hardly reached the threshold of non-relevance during the 3-month observation. A similar pattern was observed in the self-reported percentage of recovery, where participants admitted to ICU reported values $<75 \%$, while non-ICU participants reported recovery $\geq 80 \%$ of their previous capacity after 3 months.

Conclusion: Our mobile health approach provides dense recordings of symptom burden, HrQoL, and anxiety and depression scores during post-hospital recovery in COVID-19. Our findings underline the importance of early initiation of specialized rehabilitation programmes (e.g., outpatient pulmonary rehabilitation) to accelerate recovery and reduce symptoms. This applies especially to individuals requiring ICU admission. We suggest further research on functional capacity post-hospitalisation and predictors for a prolonged recovery.

Disclosure: Yves Nordmann is cofounder and Chief Medical Officer of docdok.health with a financial interest in commercialization of the docdok platform, used in this study. All other authors have no conflicts to declare.

\section{P04 \\ Effectiveness of a Long-Term Home-Based Exercise Training Program using Minimal Equipment vs. Usual Care in COPD Patients: The HOMEX-1 RCT}

\author{
A. Frei ${ }^{1}$, T. Radtke ${ }^{1}$, K. Dalla Lana ${ }^{1}$, P. Brun ${ }^{2}$, T. Sigrist ${ }^{3}$, \\ M. Spielmanns ${ }^{4}$, S. Beyer ${ }^{5}$, T. Riegler' ${ }^{2}$, G. Büsching ${ }^{3}$, S. Schöndorf ${ }^{4}$, \\ J. Braun ${ }^{1}$, R. Kunz' ${ }^{1}$, M. Puhan ${ }^{1}$ \\ ${ }^{1}$ Epidemiology, Biostatistics and Prevention Institute, University \\ of Zurich, Zürich, ${ }^{2}$ Berner Reha Zentrum, Heiligenschwendi, \\ ${ }^{3}$ Klinik Barmelweid, Barmelweid, ${ }^{4}$ Zürcher RehaZentren, Wald, \\ ${ }^{5}$ Kantonspital Winterthur, Winterthur, Switzerland
}

Introduction: A majority of COPD patients fails to maintain training after pulmonary rehabilitation (PR). Against this background, we developed the home-based, minimal-equipment HOMEX strength exercise program. The aim of the HOMEX-1 RCT was to assess the effectiveness of the program in COPD patients after completing PR over the course of one year.

Methods: COPD patients from 4 Swiss PR clinic were randomized into intervention (IG) or control group (usual care, CG). Primary outcome was change in dyspnea (Chronic Respiratory Questionnaire; CRQ) from baseline to 12 months, secondary outcomes change in exercise capacity (1-minute-sit-to-stand-test, 6-minute-walk-test), health status, exacerbations and symptoms. Adjusted linear regression models and an intention-to-treat approach were used.

Results: 123 COPD patients (IG: 61, CG: 62) were randomized; 61 females, age 66.8 (8.1) years, FEV1\% predicted 39.4 (15.4). 104 participants completed 12-months follow-up (IG: 53, CG: 51). During the study year, 76 participants experienced at least one exacerbation [2.3 (1.6)] and 68 at least one serious adverse event (135 in total). Out of the 53 IG participants, 38 conducted the HOMEX training until study end and 43 for at least 10 months. We found no difference in change of CRQ dyspnea in one year (mean difference $0.28,95 \%$ CI $-0.23-0.80, \mathrm{p}=0.27$ ). There was a 
statistically significant difference in 1-minute-sit-to-stand test repetitions favoring the IG after one year (mean difference 2.6 (95\% CI 0.22-5.03, $\mathrm{p}=0.033$ ). In all other outcomes, no significant differences between the two groups were found.

Conclusions: The majority of this multimorbid and severely ill patient group succeeded in adhering to the HOMEX training in the long-term. Our results show that the HOMEX training provided benefit in functional exercise capacity assessed by the 1-minute-sit-to-stand test. This intervention is a promising approach to maintain the effects after PR long term.

Disclosure: Nothing to disclose

\section{P05 \\ Risk Assessment of Acute Exacerbations in COPD during Pulmonary Rehabilitation Based on the Prevalence and Severity of Comorbidities}

\author{
$\underline{\text { P. Luu }}^{1}$, W. Windisch ${ }^{2}$, S. Tulka ${ }^{3}$, S. Knippschild ${ }^{3}$, M. Spielmanns ${ }^{4,5}$ \\ ${ }^{1}$ Pneumologie, Zürcher RehaZentren Klinik Wald, Wald-ZH \\ (Schweiz), Wald, Switzerland, ${ }^{2}$ Abt. Pneumologie, Lungenklinik, \\ Kliniken der Stadt Köln gGmbH, Köln, ${ }^{3}$ Institut für medizinische \\ Biometrie und Epidemiologie, Universität Witten/Herdecke, \\ Witten, Germany, ${ }^{4}$ Wald | Pneumologie, Zürcher RehaZentren, \\ Wald, Switzerland, ${ }^{5}$ University Witten-Herdecke, Witten, \\ Germany
}

Introduction: Acute COPD exacerbations (AECOPD) in the context of pulmonary rehabilitation (PR) are frequent and dangerous complications and, in addition to impairing quality of life, lead to an interruption of PR and jeopardize PR success. The question arises whether the Charlson Comorbidity Index (CCI) or the Cumulative Illness Rating Scale (CIRS) are suitable for the preventive detection of COPD patients at risk for exacerbation.

Methods: Data of COPD patients who underwent PR were analyzed with the CCI (primary endpoint). All exacerbations were recorded. 44 patients ( 22 with and 22 without exacerbation during PR) were required, CCI and CIRS were determined. CCI was evaluated by group comparison of the arithmetic means and Welch test.

In addition, the optimal cut-off point for discrimination in AECOPD and non-AECOPD patients was obtained via Recei- ver Operating Characteristic (ROC) analysis for both the CCI and the CIRS.

Results: Out of 244 COPD patients who underwent PR for an average of 21 days, 59 (24\%) suffered AECOPD that required treatment during PR. The 22 patients with AECOPD had a mean CCI of 6.77 (1.97) and the 22 patients with- out AECOPD had a mean CCI of 4.32 (1.17). This difference of -2.45 was statistically significant at a level of significance of $5 \%(p<0.001$; 95\% CI: [-3.45; $-1.46]$ ). The ROC analysis led to 6 as the optimal cut-off point for the CCI, with $81.8 \%$ sensitivity for determining an AECOPD and $86.4 \%$ specificity with an area under the curve (AUC) value of 0.87 .
The optimal cut-off point for CIRS was 19 with a sensitivity of 50\%, a specificity of $77.2 \%$ and an AUC of 0.65 .

Conclusion: AECOPD during PR have a higher CCI. The CCI allows the risk of AECOPD to be assessed with high sensitivity and specificity in partici- pants with COPD in an inpatient PR program.

Disclosure: Nothing to disclose

\section{P06 \\ Implementation Evaluation of a Home-Base Exercise Training Program for COPD Patients: A Mixed-Methods Study}

\section{A. Frei, T. Cerini, R. Kunz, K. Dalla Lana, T. Radtke, A. Polhemus, M. Puhan}

Epidemiology, Biostatistics and Prevention Institute, University of Zurich, Zürich, Switzerland

Introduction: We assessed in an RCT the effectiveness of the newly developed, home-based, minimal equipment exercise training program HOMEX in COPD patients. The aims of this study were to evaluate the implementation of HOMEX during the study year and subsequent months, considering the perspective of all involved persons, and to draw recommendations to optimize HOMEX and to initiate its sustainable implementation in additional settings.

Methods: Mixed-methods study, guided by MRC guidance on process evaluation and Proctor implementation outcomes. To assess acceptability and appropriateness we conducted a survey and interview with 50 intervention group (IG) participants at RCT end and in-depth interviews with nine compliant IG participants a few months later. We conducted semi-structured interviews with seven coaches and six potential future providers to learn about context factors and future settings. To assess reach, dose, fidelity and adoption we summarized formally reported information (e.g. training logs, coaches' reports), complemented with results from qualitative assessments.

Results: Out of the 53 IG participants, 38 conducted the HOMEX training until study end and 43 for at least 10 months. On average, the 53 participants trained during 37 out of 52 weeks. The most important facilitators for the patients' long-term motivation were experienced improvement in strength, supervision by the coach and integration of the training in daily routine. The patients perceived the acceptability of HOMEX as high, with the homebased aspect as a particular strength and the exercises as appropriate and relevant for rehabilitation. The coaches perceived the exercise cards, well-selected exercises and home visits as most relevant. Based on the stakeholder interviews, we are currently planning the implementation of the HOMEX program in three settings.

Discussion: This study provides orientation for a successful and sustainable implementation of interventions assessed in trials into practice beyond the study setting.

Disclosure: Nothing to disclose 


\section{Infections, Tuberculosis, Cystic Fibrosis/ Non-CF Bronchiectasis}

\section{P07 \\ Induced Pluripotent Stem Cell Derived Lung Alveolar Organoids}

\author{
V.B. Ozan ${ }^{1,2}$, A. Gazdhar ${ }^{1,2}$, T. Geiser $^{1,2}$ \\ ${ }^{1}$ Department of Pulmonary Medicine, Inselspital, Bern \\ University Hospital, University of Bern, ${ }^{2}$ DBMR, University \\ of Bern, Bern, Switzerland
}

Background: Idiopathic pulmonary fibrosis (IPF) is a chronic lung disease with high morbidity and mortality for which novel therapeutic options are urgently needed. One of the major limitations to identify new drug candidates is the lack of representative in vitro and in vivo models and shortage of patient material, specifically alveolar epithelial cells. Induced pluripotent stem cells (iPSCs) offer a promising novel technique to generate patient and disease-specific cells. iPSCs can be differentiated and grown in 3D culture to organoids that can be further used for basic research, disease modelling and drug screening. We aim to recapitulate the complex microenvironment of the alveoli in vitro by combining alveolar epithelial cells and endothelial cells to form lung alveolar organoids.

Methods: iPSCs cells were grown and differentiated into Alveolar epithelial cells as described before (Tamo L et al 2018). iPSCs were differentiated into endothelial cells by chemical induction. Each stage of differentiation was validated via flow cytometry and microscopy.

Results: iPSCs cultured in defined media were first differentiated into Definitive Endoderm as confirmed by positive staining for SOX2 and NKX2.1; media was changed directing the cells towards Anterior Foregut Endoderm (AFE), cells stained positive for NKX2.1 and SOX 9 confirming AFE differentiation. AFE were differentiated into Alveolar Epithelial Type II Cells (AECII) and AECII were further differentiated into AEC type I. AECI and AECII were grown in different concentrations of matrigel to generate self-organizing alveolar organoids. iPSC were separately differentiated via chemical induction into endothelial cells validated by CD31 staining.

Conclusion: iPSC differentiate into alveolar epithelial cells and endothelial cells; the AECI and AECII develop self organizing organoids when grown in matrigel.

Disclosure: Nothing to disclose

\section{P08 \\ Video-Observed Therapy for Treating Tuberculosis: A Proof of Concept Study}

\author{
J.-P. Janssens ${ }^{1}$, A. Vaudaux ${ }^{2}$, C. Lhonneux ${ }^{2}$, A. Sadowski ${ }^{2}$, \\ C. Maltor ${ }^{2}$, H. Takahashi ${ }^{2}$, D. Adler ${ }^{3}$, P. Gasche ${ }^{1}$ \\ 'Department of Medicine, Geneva University Hospital (HUG), \\ ${ }^{2}$ Ligue Pulmonaire Genevoise, ${ }^{3}$ Department of Medicine, \\ Geneva Medical School, Geneva, Switzerland
}

Introduction: Directly observed therapy (DOT) has been recommended by the World Health Organization (WHO) since the 1980 's to enhance adherence to treatment and treatment completion for tuberculosis. Because DOT can be tedious for the patients and time-consuming for health care workers (HCW), using cellphones for transmitting short videos has been proposed as an alternative to DOT (A. Story, Lancet, 2019). Video-observed therapy (VOT) has not yet been implemented in Switzerland.

Methods: With the Geneva Pulmonary League, we launched a "proof of concept" study using the SureAdhere platform (SureAdhere, San Diego, CA, USA) combined with the sureAdhere V2 application for android cellphones. This anonymized platform allows the patient to send short video sequences with a cellphone showing him/her 1/ provide name, date and time of the day, 2/ take the treatment in a clearly visible way, and $3 /$ send a message to the health care team regarding possible side effects or discomfort. Our aim was to include 10 patients over 4 months.

Results: To date, 8 patients have been included. All were either already on DOT, or just beginning their treatment. Training is relatively easy as well as use of the cellphone application. Training was provided either during hospitalization, or as outpatient. The cellphone application is uploaded by/with the nurses; a password is generated by the platform, and a first trial can be performed by the patient with the trainer. Problems encountered have been: quality of videos (1 case), language barriers (which require specific strategies; the platform is planning to adapt to different languages), non adherence (1 case).

Conclusion: VOT is an interesting and promising alternative to DOT, and can be very helpful when distances to treatment center are a problem. In this small proof of concept study, acceptance by patients is good, with a potential time-saving for patients and nurses.

Disclosure: Nothing to disclose
SSP/SSTS - EFP

Joint Annual Conference 2021 


\section{P09 \\ Disease Severity Adjusted Inpatient COVID-19 Mortality at the First and Second Wave}

\author{
P.-O.Bridevaux ${ }^{1,2}$, G. Gex ${ }^{1}$, I. Frésard' ${ }^{1}$, L. Genecand ${ }^{1}$, N. Gobin ${ }^{3}$, \\ R. Bouali ${ }^{4}$ \\ ${ }^{1}$ Service de Pneumologie, Centre Hospitalier du Valais Romand, \\ Sion, ${ }^{2}$ Service de Pneumologie, University of Geneva, Geneva, \\ ${ }^{3}$ Service de Médecine Interne, ${ }^{4}$ Direction Médicale, Centre \\ Hospitalier du Valais Romand, Sion, Switzerland
}

Background: Second wave COVID-19 mortality may have been reduced in hospitalized patients by dexamethasone, targeted use of high-flow ventilation techniques or non invasive or mechanical ventilation.

Aims and objectives: To compare mortality of the first and second wave of COVID-19, adjusted for validated risk factors associated with mortality.

Methods: Consecutive COVID-19 positive patients admitted at the Hôpital du Valais were included until discharge or death. A modified ISARIC 4C score (not including Glascow coma scale), extracted from electronic data, was chosen to adjust for the risk of death. Missing data for ISARIC were filled in with multiple imputation techniques.

Results: Among 1211 COVID-19 cases in 2020, 73.4\% were admitted at the second wave. Mean age was 71.1 (SD 16.4), 57.7\% were male and $5.7 \%$ had a chronic respiratory disease (CRD). ISARIC score, was slightly higher in the second wave indicating more severe disease. Intensive care unit (ICU) admission rate was lower at the second wave $(7.5 \%$ vs $11.8 \%, \mathrm{p}=0.02)$ alongside with shorter stay (12.3 days vs. 5.1 days). Death rates at first and second wave were $18.9 \%$ and $17.6 \%$ respectively $\mathrm{p}=0.456$ ).

The modified ISARIC $4 \mathrm{C}$ score showed an excellent prognostic value for assessing the risk of death (AUC 0.83). After adjusting for the severity of COVID-19 at admission, risk of death tended to be lower at the second wave compared to first one (OR 0.69 [0.46 1.03] $\mathrm{p}$ 0.07). Having a CRD was associated with higher risk of death (adjusted OR 2.82 [1.41- 5.65].

Conclusions: Despite higher mean ISARIC 4C score and less ICU admission, the mortality of the second wave tended to be lower compared to the first wave suggesting improvement of care.

Disclosure: Nothing to disclose

\section{P10 \\ Time to Detection of Growth for Mycobacterium Tuberculosis (MTB) in a Low Incidence Area}

\author{
R. Vongthilath-Moeung ${ }^{1}$, A. Poncet ${ }^{2}$, G. Renzi ${ }^{1}$, J. Schrenzel', \\ J.-P. Janssens ${ }^{1}$ \\ ${ }^{1}$ Department of Medicine, ${ }^{2}$ Department of Health \\ and Community Medicine, Geneva University Hospital (HUG), \\ Geneva, Switzerland
}

Background: The diagnosis of tuberculosis has improved dramatically since the widespread use of highly sensitive PCR $\mathrm{X}$-pert systems. However, some cases remain PCR negative, or cannot have PCR or microscopy because of small sample size. For these cases, time to detection of growth (TDG) is a crucial information for the clinician.

Methods: Retrospective analysis (2015-2020) of a database including all cultures for mycobacteria at Geneva University Hospital. All culture positive samples for MTB with either no PCR or a negative PCR were analyzed. Samples incubated after more than 24 hours of TB treatment were excluded from the analysis.

Results: Among 38065 samples analyzed during the study period, 236 were culture positive for MTB from 147 patients. Origin of samples was cavitary pulmonary TB $(73,31 \%)$, pulmonary non-cavitary TB $(116,49 \%)$ or extra-pulmonary TB $(47$, $20 \%$ ). Microscopy was positive in 49 cases (78 samples, 33\%). Median (IQR) TDG (table 1) for all samples was 25 (17;40) days. TDG was $>28$ days in $43 \%$ of samples. It was significantly shorter in smear positive samples and cavitary pulmonary TB. For smear negative samples, median TDG was $30(23 ; 44)$.

Conclusions: A substantial proportion of samples (43\%) became positive after 4 weeks. TDG was shorter in smear positive and/or cavitary TB samples. These results do not confirm prior studies which suggested that for the vast majority of MTB samples, TDG was < 28 days (GE Pfyffer, J Clin Microbiol, 2012; Tyrrel, FC et al; J Clin Microbiol 2012).

Disclosure: Nothing to disclose

\begin{tabular}{|c|c|c|c|c|c|}
\hline Time to detection of growth (in days) & Mean (SD) & Median (IQR) & 90th centile & TDG $>28$ days, $n(\%)$ & $\mathrm{P}$ value \\
\hline All samples $(n=236)$ & $31(21)$ & $25(17$ to 40$)$ & 55 & $101(43)$ & \\
\hline Clinical presentation & & & & & $<0.001$ \\
\hline Respiratory samples from non-cavitary TB $(n=116)$ & $35(19)$ & $29(21$ to 45$)$ & 57 & $61(53)$ & \\
\hline Respiratory samples from cavitary TB $(n=73)$ & $21(19)$ & $15(11$ to 25$)$ & 42 & $16(22)$ & \\
\hline Extrapulmonary samples $(n=47)$ & $35(24)$ & $28(21$ to 40$)$ & 78 & $24(51)$ & \\
\hline Smear & & & & & $<0.001$ \\
\hline Positive $(\mathrm{n}=78)$ & $20(19)$ & $13(11 ; 22)$ & 35 & $10(13)$ & \\
\hline Negative $(\mathrm{n}=152)$ & $36(19)$ & $30(23 ; 44)$ & 58 & $90(59)$ & \\
\hline
\end{tabular}

Time to detection of growth (in days). 


\section{P11}

\section{Self-Proning in COVID-19 Patients on Low-Flow Oxygen Therapy: A Cluster Randomised Controlled Trial}

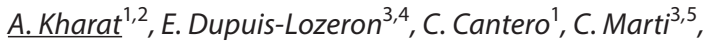
O. Grosgurin 5 , S. Lolachi ${ }^{5}$, F. Lador ${ }^{1,3}$, J. Plojoux ${ }^{1,3}$, J.-P. Janssens ${ }^{1,3}$, P.M. Soccal ${ }^{1,3}$, D. Adler ${ }^{1,3}$

${ }^{1}$ Division of Lung Diseases, Geneva University Hospitals, Geneva, Switzerland, ${ }^{2}$ Service de Soins intensifs, Centre Hospitalier de I'Université de Montréal, Montreal, QC, Canada, ${ }^{3}$ Geneva Medical School, ${ }^{4}$ Division of Clinical Epidemiology, ${ }^{5}$ Division of Internal Medicine, Geneva University Hospitals, Geneva, Switzerland

Introduction: Prone positioning as a complement to oxygen therapy to treat hypoxemia in COVID-19 pneumonia has been widely adopted even in non-severe patients, despite a lack of evidence. The aim of this single center cluster randomised trial is to test the hypothesis that an invitation to self-prone would decrease oxygen needs in patients admitted to the ward for COVID-19 pneumonia on low-flow oxygen therapy.

Methods: 27 patients with COVID-19 pneumonia admitted to our hospital were included in the study. 10 patients were randomised to self-proning and 17 to usual care.

Results: Oxygen needs assessed by oxygen flow on nasal cannula at inclusion were similar between groups. Time from first symptoms to inclusion was $10.5 \pm 5.1$ days. Estimated average time spend in prone-position was $295 \mathrm{~min} \pm 216 \mathrm{~min}$. 24 hours after starting the intervention, the median oxygen flow was $1.0 \mathrm{~L} / \mathrm{min}$ (interquartile range (IQR), 0.1-2.9) in the prone position group and $2.0 \mathrm{~L} / \mathrm{min}$ (IQR, 0.5-3.0) in the control group $(P=0.507)$ (figure 1: upper panel). Median oxygen saturation/fraction of inspired O2 ratio was 390 (IQR, 300-432) in the prone position group and $336(\mathrm{IQR}, 294-422)$ in the control group $(P=0.633)$ (figure 1: lower panel). Self-prone positioning was easy to implement and well tolerated. No serious side effect was reported.
Fig. 1. $\mathrm{O} 2$ flow (upper panel) and $\mathrm{SpO} 2$ : FiO2 (lower panel) in the self-prone group and in the control group.
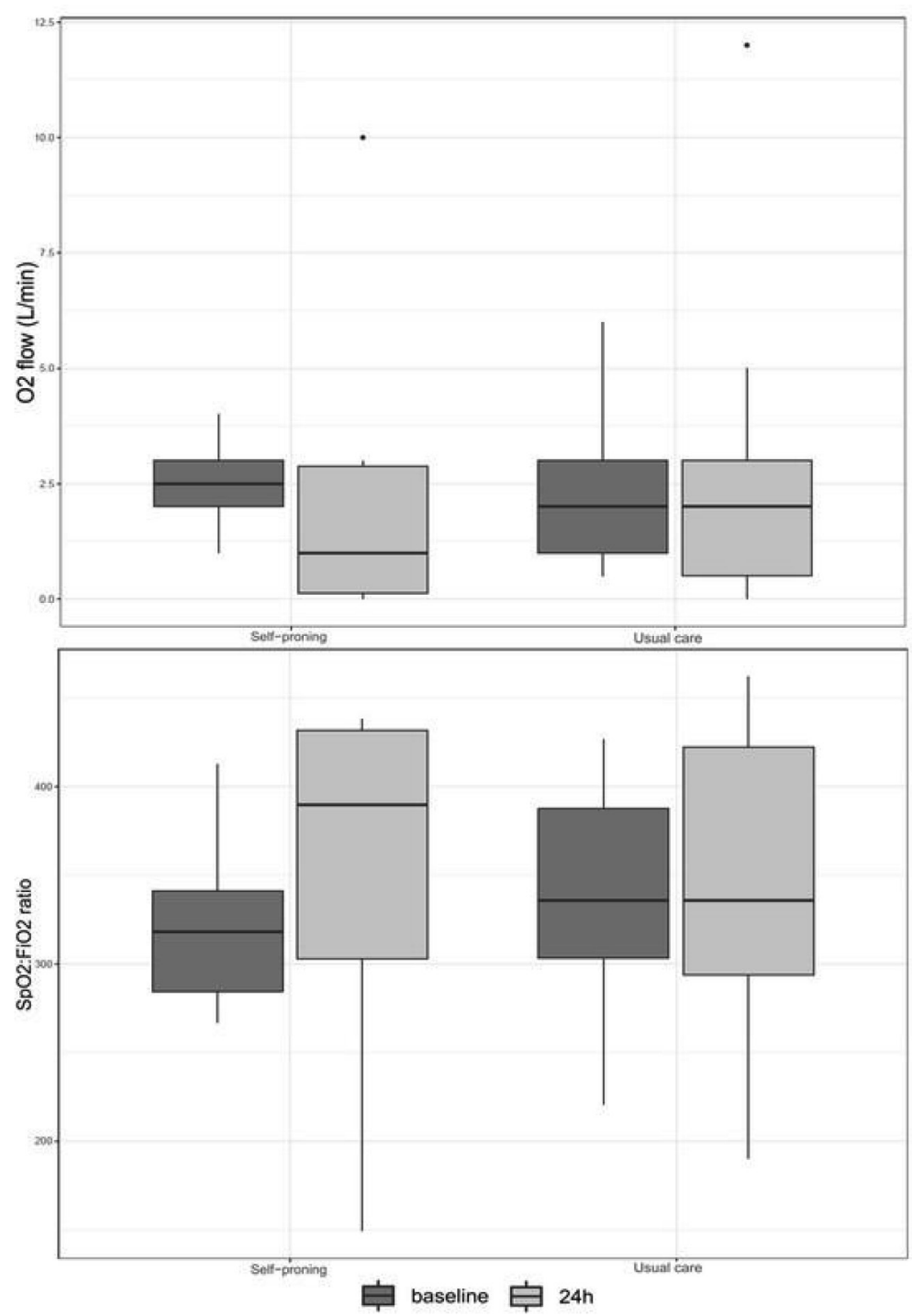
Conclusion: Self-prone positioning in patients with COVID-19 pneumonia requiring low-flow oxygen therapy resulted in a clinically meaningful reduction of oxygen flow, but without reaching statistical significance. Early interruption of the trial probably resulted in underpower of our study. With the increase of COVID-19 cases, any simple intervention to limit the progression of hypoxemia and avoid transfers to ICUs may be of benefit for the management of hospital resources.

Disclosure: Nothing to disclose

\section{P12 \\ Nontuberculous Mycobacteria under Scrutiny in the Geneva Area (2015-2020)}

\author{
R. Vongthilath-Moeung ${ }^{1}$, J. Plojoux ${ }^{1}$, A. Poncet ${ }^{2}$, G. Renzi ${ }^{3}$, \\ N. Veziris ${ }^{4,5,6}$, J. Schrenzel/,7, J.-P. Janssens ${ }^{1}$ \\ ${ }^{1}$ Division of Pulmonary Diseases, ${ }^{2}$ Department of Health \\ and Community Medicine, ${ }^{3}$ Bacteriology Laboratory, \\ Geneva University Hospitals, Geneva, Switzerland, ${ }^{4}$ Centre \\ d'Immunologie et des Maladies Infectieuses, Sorbonne \\ Universite, ${ }^{5}$ Laboratoire de Bacteriologie-Hygiène, ${ }^{6}$ Centre \\ National de Référence des Mycobactéries, APHP. Sorbonne \\ Université, Paris, France, ${ }^{7}$ Division of Infectious Diseases, \\ Geneva University Hospitals, Geneva, Switzerland
}

Background: Nontuberculous mycobacteria (NTM) are increasingly identified in industrialized countries and their role as pathogens is increasing. The relative prevalence of NTM strains show an important geographical variability. This establishing the local relative prevalence of NTM strains is important for clinicians.

Methods: Retrospective analysis (over a 5-year period: 20152020) of a comprehensive database including all results of cultures for mycobacteria in a University Hospital (Genava, Switzerland) covering a population of approximately 500'000 inhabitants. Analysis of the relative prevalence of strains identified, characteristics of patients included, whether treated or not, associated risk factors and comorbidities.

Results: Among 38065 samples analyzed during the study period, 411 were culture positive for NTM, representing 236 strains, 231 episodes of care for 222 patients. Patients in whom NTM were identified were predominantly female (55\%), in their early 60's, with a low BMI (Median: $22.6 \mathrm{~kg} / \mathrm{m}^{2}$ ). M. avium intracellulare (MAI) complex was the most frequently identified group (37\% of strains) followed by M. gordonae (24\%) and M. xenopi (12\%) among the slowly growing mycobacteria (SGM), while the M. chelonae/abscessus group (11\%) were the most frequently identified rapidly growing mycobacteria (RGM). Only 19\% of all patients were treated, mostly for pulmonary infections: MAI were the most frequently treated NTM $(n=19,44 \%$ of patients treated) followed by RGM ( $\mathrm{n}=15,35 \%)$ and M. xenopi $(\mathrm{n}=6,14 \%)$. Among those treated, $26 \%$ were immunosuppressed, $14 \%$ had pulmonary comorbidities, and 5\% systemic comorbidities.

Conclusions: In Western Switzerland, positive cultures for NTM lead to a specific treatment in approximately $20 \%$ of subjects, mostly female, in their late 50's, with a low BMI, and a low prevalence of immunosuppression or associated severe comorbidities.

Disclosure: Nothing to disclose

\section{P13 \\ Preliminary Results of an Exploratory, Observational Cohort Study on Smartphone-Enabled Cough Detection in Patients with COVID-19}

\author{
M. Boesch ${ }^{1}$, F. Rassouli ${ }^{1}$, F. Baty ${ }^{1}$, P. Tinschert ${ }^{2,3,4}$, F. Barata ${ }^{2,3}$, \\ I. Shih ${ }^{2,3,4}$, D. Cleres $^{2,3}$, E. Fleisch ${ }^{2,3}$, M. H. Brutsche ${ }^{1}$ \\ ${ }^{1}$ Lung Center, Cantonal Hospital St. Gallen, St. Gallen, \\ ${ }^{2}$ Department of Management, Technology, and Economy, ETH \\ Zurich, Zurich, ${ }^{3}$ Centre for Digital Health Interventions, ETH \\ Zurich \& University of St. Gallen, Zurich \& St. Gallen, ${ }^{4}$ Resmonics \\ AG, Zurich, Switzerland
}

Introduction: Cough is a major symptom of COVID-19. Elderly people and people with pre-existing medical conditions such as diabetes and hypertension are at increased risk of hospitalization and death. Mobile technology could help predict the course of disease more accurately. We set out to detect cough frequencies in patients hospitalized for COVID-19 and non-COVID-19 pneumonia and correlate these data to various clinical and laboratory parameters.

Method: We employed a convolutional neural network-based model for smartphone-based detection of cough in 33 patients with COVID-19 and 12 patients with non-COVID-19 pneumonia in a non-ICU setting. Clinical and laboratory parameters were extracted from corresponding medical records and analysed for potential associations with cough frequencies.

Results: Coughing episodes could be reliably detected in COVID-19 and non-COVID-19 patients over extended periods of time (days to weeks). In COVID-19, but not in non-COVID-19 pneumonia, hourly cough counts were positively associated with blood ferritin levels, $\mathrm{FiO}_{2}$, and breathing rate; in contrast, no correlation with markers of clotting was found. Hourly cough counts were negatively associated with the length of hospital stay both in COVID-19 and non-COVID-19 settings.

Conclusion: Smartphone-enabled quantification of cough is feasible in hospitalized patients with COVID-19 and other lower respiratory tract infections. Although cough frequencies varied greatly between individuals, significant associations of cough counts with surrogate markers of COVID-19 disease activity were found. Cough counts were differentially associated with clinical and laboratory parameters in COVID-19 and non-COVID-19 pneumonia. Even though our study is limited by a relatively small sample size, results are encouraging and pave the way for further investigation of cough frequencies as a novel digital-predictive marker for COVID-19.

Disclosure: Nothing to disclose 


\section{P14 \\ Pneumocystis Jirovecii and Children Interstitial Lung Disease, a Coincidence?}

I. Rochat, V. Pereitti, A. Fouraki, S. Guerin, P.A. Crisinel, S. Blanchon

Departement Femme Mere Enfant, CHUV - Centre Hospitalier Universitaire, Lausanne, Switzerland

Pneumocystis jirovecii (Pj) pneumonia, an opportunistic infection, occurs in immunocompromised hosts. In childhood, HIV infection, malignancies, immunosuppressive treatment and primary immune deficiencies are known risk factors. $P j$ colonization in immunocompetent children is common, as evidenced by the presence of $P j$ antibodies in more than $2 / 3$ of healthy children by the age of 4 years. The significance of such a finding is not well known. For example, an association between mild $P j$ infection and sudden infant death syndrome has been described, without evidence of direct causality. In adults, high prevalence of $P j$ was noted in patients with underlying lung disease, pointing to its potential role in the occurrence of pulmonary diseases. The same has not been put forward in children. There might be other unrecognized clinical manifestation of $P j$ different from the overwhelming pneumonitis, in particular in children with interstitial lung disease (chILD). We describe the cases of two female infants (4.5 months and 8 months) born at term, with negative newborn screening. Both were referred due to persistent tachypnea, one since 2 months of age, the other from birth. Both displayed normal growth, there was no other clinical sign, lung auscultation was clear, oxygen saturation was normal. Chest CT-scans were mildly contributory. Bronchoalveolar lavage (BAL) showed normal cellularity, negative microbiology except positive Pj PCR in low titers (400 copies $/ \mathrm{ml}$, and 800 copies $/ \mathrm{ml}$ ) in both specimen. Extensive work up for immune deficiency and chILD was undertaken and is under way. Treatment with co-trimoxazole resulted in no improvement. The presence of $P j$ PCR in small counts in BAL complexifies the management of chILD; due to its weak positive predictive value, $P j$ might be considered a bystander. On the other hand, chILD could impair the host resistance and contribute to low grade $P j$ pneumonia, broadening the clinical spectrum of this low virulence pathogen.

Disclosure: Nothing to disclose

\section{P15 \\ Covid 19 in the Pulmonary Department during the Containment Period}

\section{S. Abdala, S. Aitbatahar, L. Amro}

Department of Pneumology, Mohamed VI University Hospital Center, Marrakesh, Morocco

Introduction: In Morocco, although decontainment is underway with the relaxation of barrier measures, new cases of Covid 19 recorded daily by the Ministry of Health continue to increase.

Objective: To study the prevalence of Covid 19 in patients hospitalized for lung injury after containment.

Methods: Prospective epidemiological study from June 15, 2020 to the january 2021 including patients hospitalized in the pneumology department of CHU Mohamed VI university Hospital of Marrakesh, who received a COVID 19 PCR. We used 50\% of the department's bedding capacity at a rate of one patient per room in the context of total compliance with the distancing measures.

Results: A total of 134 patients were hospitalized, the causes found in our patients were: bronchial carcinoma (40.8\%), pneumothorax (18.9\%), pleuropulmonary tuberculosis (7.4\%), diffuse interstitial lung disease (7.6\%), pneumonia (5.5\%), pyothorax (4\%), hydropneumopthorax (3\%), pulmonary embolism $(3 \%)$, acute exacerbation of COPD (2.23\%), bronchial dilatation, pulmonary metastasis of extra thoracic neoplasia, pulmonary aspergilloma of which 2 cases each and a hydatid cyst in one patient. All our patients were tested for covid 19 PCR, nine patients were positive $(6.7 \%), 66.6 \%$ of whom had presented bronchial carcinoma, $22.2 \%$ with an acute exacerbation of IPF, one pneumothorax, one pulmonary embolism and one pneumonia in each case. The management was based on the transfer of all our patients to IBN Tofeil hospital Covid 19, for isolation, start of the therapeutic protocol associating hydroxyclorochin and azithromycin, heparin and oxygen therapy, while monitoring their clinical and biological status; the evolution was good in all our patients, after their recovery the patients were convened in our training for a complementary management of their pulmonary pathology.

Conclusion: The good management and the respect of hygiene and distancing measures by the nursing staff of the pneumology department during the decontamination period avoided the contamination and the propagation of the Covid 19 infection within our department.

Disclosure: Nothing to disclose 


\section{P16 \\ Clinical, Biological and Development Aspects in Geriatric Patients Infected with COVID 19}

\section{S. Abdala, S. Aitbatahar, L. Amro}

Department of Pneumology, Mohamed VI University Hospital Center, Marrakesh, Morocco

Introduction: Since the epidemic of the new coronavirus 2019 (COVID-19), which has spread rapidly worldwide, geriatric people were more likely to develop a more severe forms than younger people. Our study focused on older patients with COVID-19 and we analyzed the clinical, paraclinical and evolutive epidemiological characteristics.

Methods: We collected 34 patients over 50 years of age, admitted and managed in the pneumology department at the hospital arrazi of the CHU Mohamed VI of Marrakech during the period from March to June 2020. We studied the epidemiological, clinical, biological and evolutive characteristics of these patients.

Results: $60 \%$ of the elderly patients were male, the age groups of 50 to 64 years represented $64.7 \%, 65$ to 79 years $29.3 \%$ and over 80 years $6 \%$. Among the antecedents we noted hypertension in $29.4 \%$, diabetes in $17.6 \%$, smoking in $8.8 \%$, dysthyroidism in $5.8 \%$, asthma and ulcerative colitis in $3 \%$ of cases. Fever $(78.3 \%)$, cough (56.7\%), dyspnea $(30.0 \%)$ and asthenia (23.3\%) were the common symptoms of COVID-19 infection, $23.5 \%$ of the cases presented with respiratory distress. On paraclinical tests, we noted hyperleukocytosis in $32.5 \%$, lymphopenia in $44 \%$, leukopenia in $7 \%$, eleveted CRP (58.8\%), d-dimer level above $500 \mathrm{ug} / \mathrm{dl}$ (44\%), with high fibrinogen $(38.2 \%)$ and $\mathrm{LDH}(50 \%)$. All patients were put on hydroxychlorochin with erythromycin in addition to antibiotic therapy in $23.5 \%$, eight patients were admitted to the intensive care unit, and the mortality rate was $8.3 \%$.

Conclusion: In the light of these results we could conclude that age and comorbidities mainly diabetes and immunosuppression were associated with a high mortality rate in covid 19.

Disclosure: Nothing to disclose

\section{Interstitial and Rare Lung Diseases}

\author{
P17 \\ Adoptive Transfer of Hepatocyte Growth Factor \\ Expressing T Cells as Potential Therapeutic \\ Approach in Bleomycin Injured Mouse Lung

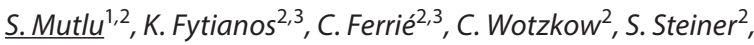 \\ A.-B. Tschirren 2,3, A. Gazdhar ${ }^{2,3}$, F. Blank ${ }^{2,3}$ \\ 'Department of Biomedical Research, Department \\ of Pulmonary Medicine, Inselspital, Bern University Hospital, \\ University of Bern, Switzerland, ${ }^{2}$ Department of Biomedical \\ Research, Department of Pulmonary Medicine, Bern University \\ Hospital, University of Bern, ${ }^{3}$ Department of Pulmonary \\ Medicine, Inselspital, Bern University Hospital, University \\ of Bern, Bern, Switzerland
}

Introduction: Idiopathic pulmonary fibrosis (IPF) is a lethal disease emerging from abnormal wound repair mechanisms provoking the loss of alveolar epithelial integrity. The immune system may have an impact during the course of the disease. Our group and others, showed a significant alveolar epithelial recovery after treatment with hepatocyte growth factor (HGF). Here, we aim to test if adoptive transfer of HGF overexpressing T cells may induce immune cell homeostasis promoting alveolar epithelial repair in a murine bleomycin injured lung model.

Methods: Adult mice were administered 1.52U/kg Bleomycin in the trachea, 7 days later mice were treated with either $5 \times 10^{5}$ $\mathrm{CD}^{+} \mathrm{T}$ cells or $5 \times 10^{5}$ HGF-transfected $\mathrm{CD}^{+} \mathrm{T}$ cells were instilled intratracheally. Single cell suspensions of lung parenchyma (LP) and bronchoalveolar lavage fluid (BALF) were analyzed by flow cytometry, 7 days after treatment. BALF HGF level and lung collagen content were measured.

Results: Bleomycin treatment decreased pulmonary immune cells frequencies, such as Tregs, $\mathrm{CD}^{+} \mathrm{T}$, dendritic cells and macrophages, 14 days after administration. Adoptive transfer of HGF expressing $\mathrm{T}$ cell increased frequencies of the same populations. BALF HGF concentration in mice with adoptive transfer treatment increased and lung collagen was significantly decreased compared to mice in the control group.

Conclusion: Adoptive transfer of HGF-transfected $\mathrm{CD}^{+} \mathrm{T}$ cells lead to increased HGF level in BALF. Furthermore, recovery of different pulmonary immune cell frequencies and decrease of lung collagen content suggests $\mathrm{T}$ cell adoptive transfer as a novel therapeutic approach for treatment of lung fibrosis.

Disclosure: Nothing to disclose 


\section{P18 \\ Respiratory Symptoms of Swiss People with Primary Ciliary Dyskinesia}

\author{
M. Goutaki ${ }^{1,2}$, L. Hüsler ${ }^{1}$, Y.T. Lam ${ }^{1}$, E. Collaud ${ }^{1}$, H. Koppe', \\ E. Pedersen ${ }^{1}$, C. Kuehni, ${ }^{1,2}$ \\ ${ }^{1}$ Institute of Social and Preventive Medicine, ${ }^{2}$ Paediatric \\ Respiratory Medicine, Children's University Hospital of Bern, \\ University of Bern, Bern, Switzerland
}

Introduction: Clinical data on primary ciliary dyskinesia (PCD) are limited and mostly derived from chart reviews, leading to missing and unreliable information on symptoms. The FOLLOW-PCD standardised questionnaire allows for prospective collection of data reported directly from patients and parents of children with PCD.

Methods: We sent a questionnaire, based on FOLLOW-PCD, to all patients registered in the Swiss PCD Registry. We assessed the prevalence and severity of respiratory symptoms during the past 3 months.

Results: We received a completed questionnaire from 72 out of 92 patients (82\%) (age range 3-73 years; median 24). 51 (71\%) were adults ( $\geq 18$ years) and $39(55 \%)$ were female. 68 patients $(94 \%)$ reported chronic nasal symptoms, mainly runny $(67 \%)$ or blocked nose $(54 \%)$ or anosmia (38\%). Nasal symptoms persisted all the time in 46 patients (64\%). 42 patients (58\%) reported ear pain; 9 almost daily. Almost all patients (99\%) reported cough, 57 with frequent sputum (79\%). Cough was worst during the day in 54 patients (75\%) and occurred daily in 53 (74\%). Common problems caused by cough were reflux in $11(15 \%)$, vomiting in $7(10 \%)$ and urinary incontinence in $6(8 \%)$ patients. 9 patients $(13 \%)$ reported frequent wheeze, usually during infections or exercise and 15 patients (21\%) reported frequent shortness of breath; 7 even at rest or during daily activities.

Conclusion: This is the first study that describes patient reported symptoms in PCD. Repeated standardised clinical data will allow us to better characterize the phenotypic variability of the disease and study disease course and prognosis.

Funding: Lung League of Bern, SNF PZ00P3_185923

Disclosure: Nothing to disclose

\section{P19 \\ Importance of Comprehensive Primary Ciliary Dyskinesia (PCD) Diagnostics - Experience of the First 100 Patients at the PCD-UniBe Diagnostic Center}

\author{
L. Müller ${ }^{1}$, S.T. Savas ${ }^{1}$, S. Tschanz ${ }^{2}$, E. Kieninger ${ }^{1}$, M. Bullo' ${ }^{1}$, \\ C. Casaulta ${ }^{1}$, P. Latzin ${ }^{1}, P C D$-UniBe study group \\ ${ }^{1}$ Pediatric Pneumology, University Hospital Bern, ${ }^{2}$ Insitute \\ of Anatomy, University of Bern, Bern, Switzerland
}

Primary ciliary dyskinesia (PCD) is a rare genetic disorder characterized by immotile/dyskinetic respiratory cilia. Patients suffer from respiratory symptoms since birth. Since there is no gold standard test, diagnosis is challenging and requires different methods and a lot of expertise. Therefore, we established the comprehensive PCD-UniBe diagnostic center.

Our diagnostic approach includes nasal nitric oxide measurement, nasal brushing and air-liquid-interface (ALI) cell culture for all patients with subsequent analysis of ciliary motility by highspeed-video-microscopy (HSVM) and immunofluorescence staining (IF) of structural proteins. In selected patients, we perform electron microscopy (TEM) of the ciliary ultrastructure and genetic analysis. An interdisciplinary PCD-board brings the results to a diagnosis.

Between 01/2018 and 04/2020, we were assigned the first 100 cases from all over Switzerland (mean age 12.9a, median 5.6a, range: 16 days to $68.9 \mathrm{a}, 17$ adults). For all cases, we performed fresh HSVM and cell culture ( $90 \%$ success rate). Cell cultures provide samples without secondary effects, more cell groups and cilia to analyze. We performed HSVM ALI on successful cultures and IF on all cases (51x fresh, 78x ALI, some both). For 29 patients we performed TEM (27x ALI, 5x fresh; $3 \mathrm{x}$ both) and genetic panel analysis. The use of cell cultures dramatically reduced inconclusive findings for HSVM ( $37 \%$ fresh versus $12 \%$ ALI) and IF (53\% versus $15 \%)$. We did not find any evidence of PCD in 78 cases, diagnosed PCD in 18 patients and further analyses are ongoing in four patients. HSVM was diagnostically significant in twelve, IF in 14, TEM in five, and genetics in ten cases.

This indicates that none of the methods alone was sufficient to diagnose all 18 PCD cases and that a comprehensive approach is essential for accurate PCD-diagnosis. Cell culture was crucial and prevented re-brushings in $90 \%$ of cases. This is particularly important in pediatric patients.

Disclosure: Nothing to disclose 


\section{P20 \\ Lung Function in Adult Patients with Osteogenesis Imperfecta: A Retrospective Cohort Study}

\section{A. Lenoir ${ }^{1}$, B. Rozier Aubry ${ }^{2}$, C. Paquier ${ }^{3}$, J. Tanniger ${ }^{3}$, M. Faouzi ${ }^{4}$,} R. Lazor $^{1}$

${ }^{1}$ Division of Respiratory Medicine, Internal Medicine Department, ${ }^{2}$ Division of Genetic Medicine, Internal Medicine Department, ${ }^{3}$ Division of Physiotherapy, Musculoskeletal Department, ${ }^{4}$ Division of Biostatistics, Centre for Primary Care and Public Health (Unisanté), Centre Universitaire Hospitalier Vaudois, Lausanne, Switzerland

Introduction: Osteogenesis imperfecta (OI) is a hereditary disorder characterised by increased bone fragility, recurrent fractures and skeletal deformities. The responsible genes code for collagen synthesis and processing, and correct bone mineralisation. Although respiratory morbidity and mortality are increased in OI, their prevalence and mechanisms remain poorly understood.

Method: We retrospectively analysed adult OI patients with available pre-bronchodilator spirometry performed with an EasyOne spirometer. Results were interpreted using GLI 2012 references. Obstruction was defined as $\mathrm{FEV}_{1} / \mathrm{FVC}<$ lower limit of normal (LLN), and restrictive pattern as FVC $<$ LLN. Lung function changes over time were modelled through mixed linear regression.

Results: 45 patients were included (62\% women). Mean (SD) age at $1^{\text {st }}$ visit was $41(16)$ years, mean BMI was $25(6) \mathrm{kg} / \mathrm{m}^{2}$, and $67 \%$ were never-smokers. $60 \%$ had OI Sillence classification type I, $18 \%$ type III, $2 \%$ type IV , $2 \%$ type V, and type was undetermined in $18 \%$. At baseline, mean $\mathrm{FEV}_{1}$ was $2.48(0.86) \mathrm{L}$ or 87 (19)\%pred, mean FVC 3.11 (0.96) L or 91 (17)\%pred, and mean $\mathrm{FEV}_{1} / \mathrm{FVC}$ ratio $0.79(0.11)$. Pure obstructive pattern was found in 4 patients (9\%), pure restrictive pattern in $3(7 \%)$, and mixed pattern in 3 (7\%). Follow-up spirometry was available in 36 patients over a mean time of 3.6 years. Mean changes per year of follow-up $(95 \% \mathrm{CI})$ adjusted for sex, age, height and OI type were: $\mathrm{FEV}_{1}+37$ $\mathrm{ml}(13 ; 61), \mathrm{FVC}+31 \mathrm{ml}(7 ; 54), \mathrm{FEV}_{1} / \mathrm{FVC}$ ratio - $0.02(-0.05 ; 0.01)$. No association was found with OI type, smoking history, history of asthma, or biphosphonate or denosumab treatment.

Conclusion: Restrictive or obstructive ventilatory impairment occurs in a minority of OI patients. Slight increases in $\mathrm{FEV}_{1}$ and FVC were observed over time, for which the underlying collagen disease might play a role.

Disclosure: Nothing to disclose

\section{P21 \\ Prevalence of Birt-Hogg-Dubé Syndrome Determined through Epidemiological Data on Spontaneous Pneumothorax and Bayes Theorem}

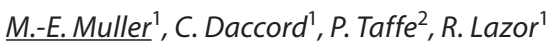

${ }^{1}$ Respiratory Medicine Department, Lausanne University Hospital and University of Lausanne, ${ }^{2}$ Division of Biostatistics, Center for Primary Care and Public Health, University of Lausanne, Lausanne, Switzerland

Introduction: Birt-Hogg-Dubé syndrome (BHD) is a rare inherited disorder characterized by cutaneous fibrofolliculomas, multiple pulmonary cysts, recurrent spontaneous pneumothorax (SP), and renal tumors. The prevalence of BHD in the general population remains unknown. This study aimed at determining indirectly the prevalence of BHD through the Bayes theorem applied to epidemiological data on SP.

Methods: we performed meta-analyses of published data on:

1) the probability of having BHD among patients with apparent primary SP (4 studies),

2 ) the incidence rate of primary SP in the general population (9 studies), and

3 ) the probability of experiencing a SP in BHD (16 studies).

Results were corrected for SP relapses, stratified by gender and year of study publication (before and after 2000), and computed with the Bayes equation.

Results: The probability of having BHD among patients with apparent primary SP was 9\% (95\% confidence interval [CI]: 7-11\%). It was 26\% (95\% CI: $18-34 \%)$ in women and 5\% (95\% CI: $4-7 \%)$ in men. The incidence rate of primary SP in the general population was 8.69 (95\% CI: 6.58-11.46) per 100'000 person-years (p-y). It was 3.44 (95\% CI: 2.36-4.99) per 100 '000 p-y in women and 13.96 (95\% CI: $10.72-18.18)$ per 100 '000 p-y in men, and was about 2 times higher in studies published after 2000 than in those published before 2000 . The probability of experiencing at least one SP among patients with BHD was 43\% (95\% CI: 31-54\%), without gender difference. By combining these data in the Bayes equation, we found a prevalence of $\mathrm{BHD}$ in the general population of 1.86 (95\% CI: $1.16-3.00)$ per million, with values of 1.86 (95\% CI: 1.02 $3.39)$ per million in men, and 1.88 (95\% CI: $0.97-3.63)$ per million in women.

Conclusion: The prevalence of BHD in the general population is about 2 cases per million, without difference between genders.

Disclosure: Nothing to disclose 


\section{P22 \\ Respiratory Symptoms and Physical Activity in Patients with Primary Ciliary Dyskinesia in Switzerland}

\author{
$\underline{\text { Y.T. Lam }}{ }^{1}$, L. Hüsler ${ }^{1}$, E. Collaud ${ }^{1}$, E. Pedersen ${ }^{1}$, C. Kuehni ${ }^{1,2}$, \\ M. Goutaki ${ }^{1,2}$ \\ ${ }^{1}$ Institute of Social and Preventive Medicine, ${ }^{2}$ Paediatric \\ Respiratory Medicine, Children's University Hospital of Bern, \\ University of Bern, Bern, Switzerland
}

Introduction: Most patients with Primary Ciliary Dyskinesia (PCD) have chronic upper and lower respiratory symptoms. We do not know if frequent respiratory symptoms result in low physical activity in PCD patients.

Methods: In a national survey, we sent questionnaires using the standardised FOLLOW-PCD questions to patients enrolled in the Swiss PCD Registry asking about patient reported symptoms and lifestyle. We used logistic regression to study if frequent (reported often or daily during the past 3 months) cough, nasal symptoms, and shortness of breath were associated with level of physical activity (less than once per week).

Results: 72 of 92 patients (82\%) completed our survey (ages 3-73 years; median 24$)$, including 51 (71\%) adults ( $\geq 18$ years) and $39(55 \%)$ female. In total, $55(76 \%)$ patients performed sports frequently, at least once per week. These included all 21 children who attended school sports or did additional sports and 34 adults. Of the 55, 21 (38\%; 4 children, 17 adults) performed fast power sports, $11(20 \%)$ adults did endurance and 11 (20\%; 6 children, 5 adults) performed game sports. The rest did other sports or did not specify. We did not find any evidence that frequent cough

(OR 2.01, 95\%CI 0.69-5.83), nasal symptoms (OR 1.57, 95\%CI $0.53-4.66$ ), and shortness of breath (OR 2.87, 95\%CI 0.87-9.51) are associated with low level of physical activity.

Conclusion: Despite frequent chronic respiratory symptoms, most patients were physically active at least once a week. A possible explanation is that Swiss PCD patients combine physical activity with physiotherapy for better airway clearance to deal with their chronic symptoms.

Funding: Lung League of Bern, SNF PZ00P3_185923

Disclosure: Nothing to disclose

\section{P23 \\ Inflammatory Activity of Stage IV Pulmonary Sarcoidosis in Patient with Stable Lung Functions: A Case Report}

$\underline{\text { S. Bengueddache }}{ }^{1}$, A. Sauty ${ }^{1}$, T. Cazaentre ${ }^{2}$

${ }^{1}$ Service of Pulmonology, Réseau Hospitalier Neuchâtelois, Neuchâtel, ${ }^{2}$ Nuclear Medicine and Radiology Units, Department of Medical Imaging, Réseau Hospitalier Neuchâtelois,

La Chaux-de-Fonds, Switzerland

Background: Stage IV sarcoidosis has been less studied than other stages of pulmonary sarcoidosis but is often considered fibrotic rather than inflammatory. Activity of the disease and treatment necessity in stage IV sarcoidosis in patients with otherwise stable clinical manifestations still need to be assessed.

Case information: A 50 years old man who reported long history of smoking and asthma presents with exertional dyspnea after two episodes of pneumococcal pneumonia. A thoracic CT scan performed during his 2nd pneumonia demonstrated images of architectural distortion of both apexes. A new examination carried out at a distance from the pneumonia confirmed these abnormalities, which were radiologically compatible with stage IV sarcoidosis. Lung function testing showed both very severe obstructive and light restrictive disorders. Symptoms, lung functions and imageries remained stables for years. Recently, the patient developed a skin lesion which biopsy confirmed the diagnosis of sarcoidosis. Surprised by this skin involvement suggestive of active sarcoidosis, a PET CT scan was performed and showed high metabolic activity regarding the known apical lesions.

Discussion/conclusion: This case shows that despite apparent clinical, functional and radiological stability, stage IV sarcoidosis may be highly active and lead to immunosuppressive therapy.

Disclosure: Nothing to disclose

\section{P24 \\ Place of Accessory Salivary Gland Biopsy in the Positive Diagnosis of Sarcoidosis}

\section{S. Abdala, S. Aitbatahar, L. Amro}

Department of Pneumology, Mohamed VI University Hospital Center, Marrakesh, Morocco

Introduction: Sarcoidosis is a systemic granulomatosis of unknown etiology. Its diagnosis is based on a series of arguments, including histology, which shows a tuberculoid granuloma without caseous necrosis. This signature can be obtained by a biopsy of the accessory salivary glands.

Material and method: We conducted a retrospective study of 30 cases of sarcoidosis hospitalized in the pneumology department of Med VI University Hospital between January 2015 and December 2020. 
Results: The average age of our patients was 48 years with a female predominance in $90 \%$ of cases. The symptomatology was dominated by dry cough in $75 \%$ of cases followed by dyspnea in $58 \%$ of cases. The extrathoracic localizations observed were: peripheral lymph node involvement in 3 cases, skin involvement in 2 cases and ophthalmic involvement in 2 cases. The histological signature was obtained by labial biopsy alone in 4 cases, staged bronchial biopsies in 7 cases, peripheral lymph node biopsy in 3 cases, echo-endoscopy, mediastinoscopy and skin biopsy in 2 cases each. In the other cases, the diagnosis of sarcoidosis was made on the basis of a combination of radioclinical and biological evidence, including conversion enzyme and BAL. Systemic corticosteroid therapy was prescribed in $70 \%$ of the cases and therapeutic abstention in $30 \%$ of the cases.

Conclusion: Salivary gland biopsy is a simple and minimally invasive procedure that can be cost-effective and help in the positive diagnosis of sarcoidosis. It also avoids the use of aggressive procedures.

Disclosure: Nothing to disclose

\section{Obstructive Lung Diseases \& Allergy, Functional Diagnostics \& Sports Pneumology}

\section{P25 \\ CPET Evidence of Dysfunctional Breathing after SARS-CoV2 in Patients with Persistent Dyspnea}

\author{
I. Frésard ${ }^{1}$, D. Lawi ${ }^{1}$, L. Genecand ${ }^{2}$, P. Vremaroiu ${ }^{1}$, \\ A. Vremaroiu Coman', G. Gex ${ }^{1}$, P.-O. Bridevaux ${ }^{1}$ \\ ${ }^{1}$ Hôpital du Valais, Sion, ${ }^{2}$ Hôpital Riviera Chablais, Rennaz, \\ Switzerland
}

Background: Post SARS-CoV2 dyspnea might persist for months after infection. Among the various causes of persistent dyspnea, dysfunctional breathing (DB) as defined as an erratic ventilation during cardiopulmonary exercise test (CPET) has been anecdotally described.

Aims and objectives: We aimed to examine the prevalence of DB among patients with resolved SARS-CoV2.

Methods: CPET was performed in 31 SARS-CoV2 patients (median age 62 [IQR 20], female sex 41.9\%) with persistent dyspnea $\geq 12$ weeks after acute infection.

CPET patterns including DB were determined by 2 pulmonologists. Non parametric and $X$ square tests were applied to analyze the association between CPET patterns and pulmonary functions tests and severity of SARS-CoV2.

Results: Three dominant patterns were identified (respiratory limitation [RL] with gas exchange abnormalities, normal CPET/ deconditioning [D] and DB). Isolated DB was found in 9 patients (29\%), while RL was found in 19 (59\%) and D in 3 (9.7\%). DB patients had significantly less severe initial infection (55.6\%), better TLCO (median [IQR] 73\% [22\%]), higher VO2 $(21.3 \mathrm{ml} /$ $\mathrm{min} / \mathrm{kg}$ [5.2]) lower VE/VCO2 slope (29.4 [5.3]) and higher SpO2 (94\%[3]) than RL patients.

Conclusions: At $\geq 3$ months after infection, one third of patients with persistent dyspnea exhibit dysfunctional breathing, which appears to be a feature of COVID-19 not described in other viral infections. We hypothesize that DB is an important physiopathological mechanism of disabling dyspnea in younger outpatients with SARS-CoV2.

Disclosure: Nothing to disclose

\section{P26 \\ Effect of Nocturnal Oxygen Therapy on Daytime Pulmonary Hemodynamics in Patients with COPD Travelling to Altitude. RCT}

\author{
M. Lichtblau, T.D. Latshang, S. Aeschbacher, F. Huber, \\ P.M. Scheiwiller, S. Ulrich, S.R. Schneider, E.D. Hasler, M. Furian, \\ K.E. Bloch, S. Saxer, S. Ulrich
}

UniversitätsSpital Zürich | Pneumologie, UniversitätsSpital

Zürich, Zürich, Switzerland

Introduction: We investigated whether nocturnal oxygen therapy (NOT) mitigates the altitude-induced increase of pulmonary artery pressure in patients with chronic obstructive pulmonary disease (COPD) when staying overnight at moderate altitude.

Methods: Patients with COPD living below $800 \mathrm{~m}$, underwent examinations at $490 \mathrm{~m}$ and during 2 sojourns at $2048 \mathrm{~m}$ (with a washout period of 2 weeks $<800 \mathrm{~m}$ between altitude sojourns). During nights at altitude patients received either NOT $(3 \mathrm{l} / \mathrm{min})$ or placebo (ambient air $3 \mathrm{l} / \mathrm{min}$ ) via nasal cannula according to a randomized cross-over design. The main outcome was the difference of the tricuspid regurgitation pressure gradient (TRPG) measured by echocardiography on the $2^{\text {nd }}$ day at altitude (performed under ambient air) between sojourns with NOT and placebo. Additional outcomes were other echocardiographic measures of the right and left heart function.

Results: Twenty-three COPD-patients (70\% GOLD II / 30\% GOLD III, mean \pm SD age $66 \pm 5$ years, FEV $_{1} 54 \pm 13 \%$ predicted) were included. TRPG significantly increased when patients travelled from $490 \mathrm{~m}$ to $2048 \mathrm{~m}(21.7 \pm 5.2 \mathrm{mmHg} ; 2048 \mathrm{~m}$ placebo $27.4 \pm 7.3 \mathrm{mmHg}$; and $2048 \mathrm{~m}$ NOT $27.8 \pm 8.3 \mathrm{mmHg}$ ) without difference between interventions. The tricuspid annular plane systolic excursion was significantly higher after NOT vs. placebo $(2.6 \pm 0.6$ vs. $2.3 \pm 0.4 \mathrm{~cm}$, mean difference ( $95 \%$ confidence interval) $0.3(0.1$ to 0.5$) \mathrm{cm}, \mathrm{p}=0.005)$.

Conclusions: NOT did not mitigate the acute effect of altitude on the TRPG in COPD lowlanders travelling to altitude compared to placebo. Whether NOT during prolonged altitude sojourns affects right heart function remains to be studied.

Disclosure: Nothing to disclose 


\section{P27 \\ Effect of Oxygen on Blood Pressure Response to Altitude Exposure in COPD - Data from a Randomised Placebo-Controlled Cross-Over Trial}

M. Meszaros ${ }^{1}$, T.D. Latshang ${ }^{1}$, S. Aeschbacher ${ }^{1}$, F. Huber ${ }^{1}$, D. Flueck ${ }^{1}$, M. Lichtblau' ${ }^{1}$, S. Ulrich ${ }^{1}$, E.D. Hasler ${ }^{1}$, P.M. Scheiwiller ${ }^{1}$, L. Reinhard ${ }^{1}$, S. Ulrich ${ }^{1}$, K.E. Bloch ${ }^{1,2,3}$, M. Furian ${ }^{1}$, E.I. Schwarz ${ }^{1,3}$

${ }^{1}$ Department of Pulmonology and Sleep Disorders Centre, University Hospital of Zurich, ${ }^{2}$ Zurich Center for Integrative Human Physiology (ZIHP), ${ }^{3}$ Centre of Competence Sleep \& Health, University of Zurich, Zurich, Switzerland

Background: Patients with chronic obstructive pulmonary disease (COPD) are particularly vulnerable to hypoxia-induced autonomic dysregulation. Hypoxaemia is marked during sleep. In COPD, altitude exposure is associated with an increase in blood pressure (BP) and blood pressure variability (BPV) as well as a decrease in baroreflex-sensitivity (BRS). Whether nocturnal oxygen therapy (NOT) may mitigate these cardiovascular autonomic changes in COPD at altitude is unknown.
Methods: In a randomised placebo-controlled cross-over trial, 32 patients with moderate-to-severe COPD living $<800 \mathrm{~m}$ were allocated to NOT or placebo during acute exposure to altitude. Measurements were done at low altitude at $490 \mathrm{~m}$ and during two stays at $2048 \mathrm{~m}$ on NOT (3 1/min) or placebo (ambient air) via nasal cannula. Allocation and intervention sequence were randomised. Outcomes of interest were BP, BRS (from beat-to-beat BP measurement), BPV, and heart rate.

Results: 23 patients finished the trial per protocol (mean(SD) age 66(5)y, $\mathrm{FEV}_{1}$ 62(14)\%predicted). The others had altituderelated adverse health effects. NOT significantly decreased the altitude-induced increase in systolic BP compared to placebo $(-10.6$ [95\%CI -20.1 to -1.0$] \mathrm{mmHg}, \mathrm{p}=0.05)$ but not diastolic BP $(-4.4$ $\mathrm{mmHg}, 95 \% \mathrm{CI}-11.4$ to $2.6 ; \mathrm{p}=0.29)$ or BPV. BRS at altitude was significantly higher in NOT than in placebo $(+1.5$ [95\%CI 0.01 to 2.95], $\mathrm{p}=0.02$ ). See figure 1 .

Conclusions: NOT may protect from hypoxia-induced autonomic dysregulation upon altitude exposure in COPD and thus protect from a relevant increase in BP and decrease in BRS. Oxygen supplementation may provide cardiovascular benefits in COPD during conditions of increased hypoxaemia. NOT may be considered in COPD travelling to altitude.

Disclosure: Nothing to disclose

Changes in systolic and diastolic BP measured by Finometer(B)
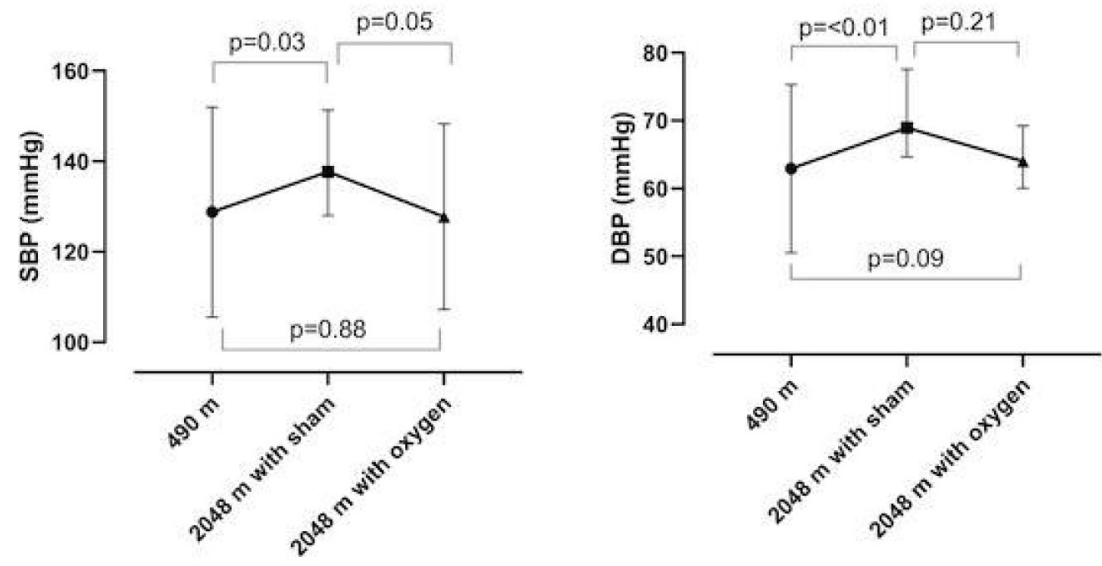

Changes in BRS and heart rate measured by Finometer ${ }^{\circ}$
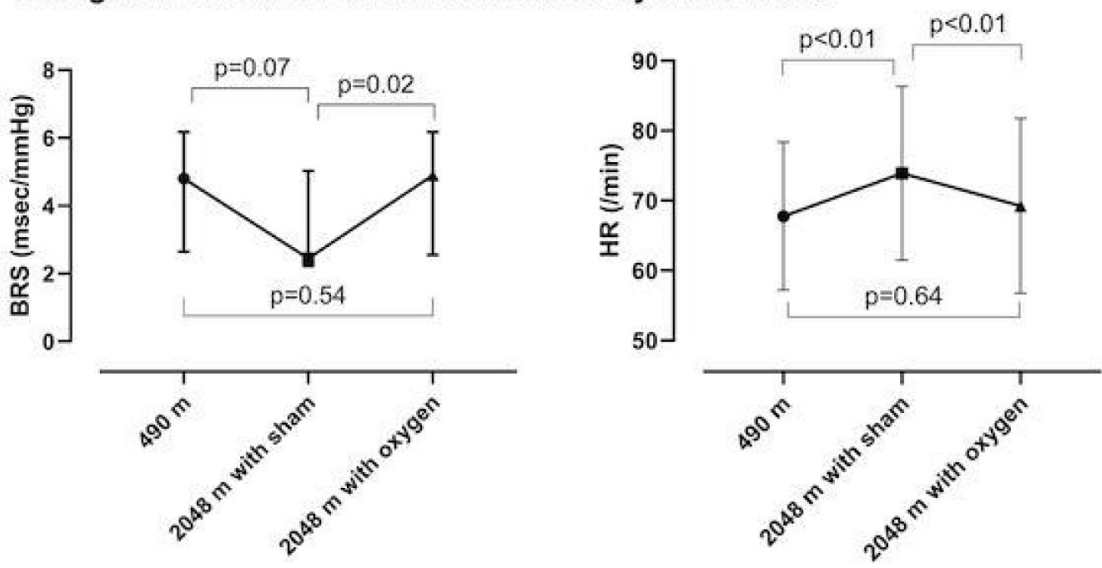

Fig. 1. 


\section{P28}

\section{Accuracy of 6 Minute Walk Test (6MWT) using a Non-Vented (NV) Mask with a Filter}

M. Hofer ${ }^{1}$, S. Beyer ${ }^{1}$, S. Rezek ${ }^{2}$, I. Unger ${ }^{2}$, J. Hetzel ${ }^{1}$

${ }^{1}$ Pneumologie, ${ }^{2}$ Physiotherapie, Kantonspital Winterthur, Winterthur, Switzerland

Aim: SARS-CoV-2 is an aerosol-transmitted disease, and it is recommended to perfomr $6 \mathrm{MWT}$ wearing a mask. In our institution, we use a NV-mask with a filter. It is unclear how comparable these results are to a normal walk test performed without mask. The aim of this study was to compare the 6MWT performed with and without a mask in a subgroup of our outpatient rehabilitation programme.

Methods: 21 patients preformed two $6 \mathrm{MWT}$ on two different days: one in the division of pneumology (PNE) with NV-mask and one in the department of physiotherapy (PHYS) without mask. As the measurements were performed in two different settings, we compared the results with 19 patients before COVID-19 who performed the $6 \mathrm{MWT}$ without a mask in both settings (control measurement).

Results: The mean age of the 21 patients was $67.8 \pm 10.1$ years. There was a highly significant difference $(\mathrm{p}=0.003)$ of the $6 \mathrm{MWT}$ performed with and without NV-mask: $367 \pm 114$ meters versus $416 \pm 110$ meters. Nevertheless, there was no significant difference in BORG after 6MWT and SO2 at start in PNE and PHYS: 4.6 \pm 2.0 (PNE) versus $4.7 \pm 2.1$ (PHYS) and $93.9 \pm 2.7 \%$ versus $93.6 \pm 2.5 \%$, respectively, but decrease in $\mathrm{SO} 2$ was significantly more pronounced in the mask group: $-7.2 \pm 6.0$ versus $-4.0 \pm 4.5 \mathrm{p}<0.01$. In the control measurement there was no significant difference in the 6MWT performed between PNE and PHYS: $363 \pm 113 \mathrm{~m}$ versus $362 \pm 117 \mathrm{~m}$.

Conclusions: 6MWT performed with and without NV-mask with filter revealed a highly significant decrease of the distance in the group with mask. There was no significant difference in BORG in the two test scenarios but there was a significant decrease of $\mathrm{SO} 2$ in the NV-mask group. The 6MWT with NV-mask seems to represent another test than the 6MWT without mask. Therefore, results of a $6 \mathrm{MWT}$ with NV-mask cannot transferred to a test without mask.

Disclosure: Nothing to disclose

\section{P29 \\ Dietary Fiber Protects against Atopic Dermatitis and Subsequent Allergic Airway Inflammation}

\author{
A. Trompette ${ }^{1,2}$, J. Pernot ${ }^{1,2}$, N. Ubags $^{1,2}$, L.P. Nicod ${ }^{2}$, \\ C. Von Garnier ${ }^{1,2}$, B.J. Marsland ${ }^{2,3}$
}

${ }^{1}$ Service de Pneumologie, CHUV, ${ }^{2}$ UNIL Faculty of Biology and Medicine, Lausanne, Switzerland, ${ }^{3}$ Monash University, Melbourne, VIC, Australia

Atopic dermatitis $(\mathrm{AD})$ is a chronic skin disease typically manifesting during the first years of life and affecting up to $20 \%$ of children in Westernized countries. Currently no cure exists. Novel therapeutic strategies are thereby desperately needed.

One main risk factor for $\mathrm{AD}$ is a decreased skin barrier function, which can lead to increased allergen ingress and consequently, establishment of a Th2-polarized milieu driving initiation and progression of allergic skin inflammation. Recently, it became evident that $\mathrm{AD}$ is not an isolated disease but rather an early inflammatory trigger. Early allergic skin sensitization can indeed predispose children to develop other allergic disorders later in life, such as allergic asthma, a succession of events known as the "atopic march".

Interestingly, products of the fermentation of dietary fiber, the metabolites short-chain fatty acids (SCFA), have been shown to alleviate diverse allergic diseases.

Here, using a mouse model and the common house dust mite (HDM) allergen, we report that dietary fiber and SCFA supplementation protect against the development of $\mathrm{AD}$, and subsequent allergic airway inflammation (AAI). Indeed, fiber-rich diet or oral SCFA ameliorated transepidermal water loss and AD severity. Sensitization to HDM was blunted as indicated by lower levels of circulating IgE antibodies. These beneficial cutaneous and systemic effects translated into an improved AAI once mice were subsequently exposed intranasally with HDM. We found that the beneficial effects of SCFA were mediated by their ability to strengthen and preserve skin barrier integrity during repeated allergen exposure. Ultimately, we unraveled that SCFA alter mitochondrial metabolism, promoting keratinocyte differentiation and production of key structural components responsible for maintaining the architecture of the cornified envelope, the outermost layer of the epidermis. Overall, our results show that dietary fiber and SCFA prevent the development of AD by improving skin barrier function, ultimately halting the dire "atopic march" sequelae.

Disclosure: Nothing to disclose 


\section{P30 \\ Modulation of Allergic Airways Disease Employing Bio-Mimetic Nanocarriers with Toll-Like Receptor Agonists}

M. Scalise ${ }^{1,2}$, C. Ferrié1, ${ }^{1,2}$, S. Mutlu ${ }^{1,2}$, M. Amacker ${ }^{3}$, C. Wotzkow $^{1}$, S. Steiner ${ }^{1}$, C. von Garnier ${ }^{4}$, F. Blank ${ }^{1,2}$

${ }^{1}$ Department for BioMedical Research DBMR, Department of Pulmonary Medicine, ${ }^{2}$ Department of Pulmonary Medicine, Inselspital, Bern University Hospital, University of Bern, Bern,

${ }^{3}$ Mymetics SA, Vaud, ${ }^{4}$ Pneumology Division, Centre Hospitalier Universitaire Vaudois, Lausanne, Switzerland

Introduction: Allergic Asthma is characterized by airway hyperresponsiveness due to a maladaptive Th2/Th9/Th17 immune response against innocuous environmental substances. Current treatments only manage to reduce the symptoms but do not alter the natural course of the disease. Therefore, there is an urgent need for more efficacious treatment approaches, aiming at the cause of the disease.

We aim to design and characterise the effects of bio-mimetic nanoparticles in a mouse model of experimental allergic inflammatory airways disease (EAIAD) to skew the Th2/Th9/Th17biased immune response towards Th1.

Methods: We established a reproducible EAIAD mouse model to treat the allergic response with liposomes or virosomes conjugated with OVA and a TLR7/8 agonist, followed by monitoring the specific immune response. Immune cells in different lung compartments (flow cytometry), as well as lung function (Flexivent System $^{\mathrm{TM}}$ ) and IgE titer (ELISA) are monitored before and after treatment.

Results: The established EAIAD mouse model analysed by flow cytometry showed an enhanced level of eosinophils in lung and lymph nodes of EAIAD mice (sensitized with OVA) compared to negative controls (saline).

Lung function data revealed that treatment with OVA-liposomes rescued animals from impaired lung function, such as enhanced airway resistance, increased forced expiratory volume in 0.1 second (FEV0.1) and reduced peak expiratory flow (PEF) as found in the non-treated OVA-sensitized and challenged positive controls. Serum IgE measurements showed enhanced levels in non-treated sensitised and challenged animals when compared to the non-sensitised control group, while treatment with OVA-virosomes and OVA-liposomes rescued animals from enhanced IgE levels.

Flow cytometry analysis of pulmonary immune cells following treatment is still in progress.

Conclusion: Our findings show that allergen and adjuvantmodified virosomes and liposomes ameliorate hallmarks of allergic airways disease. Bio-mimetic nanoparticles employed as carriers for antigen and adjuvant show great potential as future therapeutic approach for re-programming allergic airways disease.

Disclosure: Nothing to disclose

\section{P31 \\ Determination of Exercise Capacity in Long COVID Patients}

\author{
A. Bringard ${ }^{1}$, I. Guerreiro ${ }^{1}$, T. Fontolliet ${ }^{2,3}$, R. Messe ${ }^{1}$, G. Kopp ${ }^{1}$, \\ P. Gasche-Soccal ${ }^{1,4}$, F. Lador ${ }^{1}$ \\ ${ }^{1}$ Division of Pulmonary Diseases, ${ }^{2}$ Department \\ of Anaesthesiology, Clinical Pharmacology, Intensive Care \\ and Emergency Medicine, Geneva University Hospital (HUG), \\ ${ }^{3}$ Department of Basic Neuroscience, ${ }^{4}$ Department of Medicine, \\ Geneva Medical School, Geneva, Switzerland
}

Introduction: Hospitalised patients for severe COVID-19 pneumonia, with persisting respiratory symptoms 3 months after hospital discharge (long COVID), may present an altered exercise capacity. Since a plateau of $\mathrm{VO}_{2}$ is rarely achieved during conventional incremental ramp test (RAMP) (Poole et al, 2008), a supramaximal verification phase has been proposed (Rossiter et al, 2006) in order to improve confidence that parameters estimated during conventional RAMP were maximal.

Method: Long COVID patients performed a cardio-respiratory exercise test (CPET) to assess their exercise capacity. They performed RAMP followed by a supramaximal verification phase (VP) at $105 \%$ of peak-work rate (PWR) achieved during RAMP, after 20-30 min recovery. Both RAMP and VP were prolonged up to volitional exhaustion.

Results: 11 patient $(57 \pm 9$ years $)$ took part of the study. PWR achieved during RAMP was $135 \pm 35 \mathrm{~W}$, corresponding to $86 \pm 14 \%$ of predicted values. Although $\mathrm{VO}_{2}$ peak achieved during RAMP and VP were not significantly different (18.2 \pm 3.7 and $18.0 \pm 3.0 \mathrm{ml} / \mathrm{min} / \mathrm{kg}, \mathrm{p}=0.9)$, large inter-individual differences can be observed (Table 1). Two patients achieved a plateau of $\mathrm{VO}_{2}$ at the end of RAMP, but 4 patients elicited higher $\mathrm{VO}_{2}$ peak during $\mathrm{VP}$, confirming achievement of $\mathrm{VO} 2 \max$ in 7 out of 11 patients during RAMP. Finally, $\mathrm{VO}_{2}$ max was $18.7 \pm 3.1 \mathrm{ml} / \mathrm{min} / \mathrm{kg}$.

Table 1: Individual mechanical and physiological variables during RAMP and VP.

Conclusion: At 3 months after hospital discharge, long COVID patients exhibit limited exercise capacity. Application of a verification phase is well tolerated and should be proposed to confirm individually achievement of maximal physiological parameters, especially in patients with limited functional capacity.

Disclosure: Nothing to disclose 
Fig. 1. VO2 response and work rate (WR) of a representative subject (\#2) during RAMP and VP.

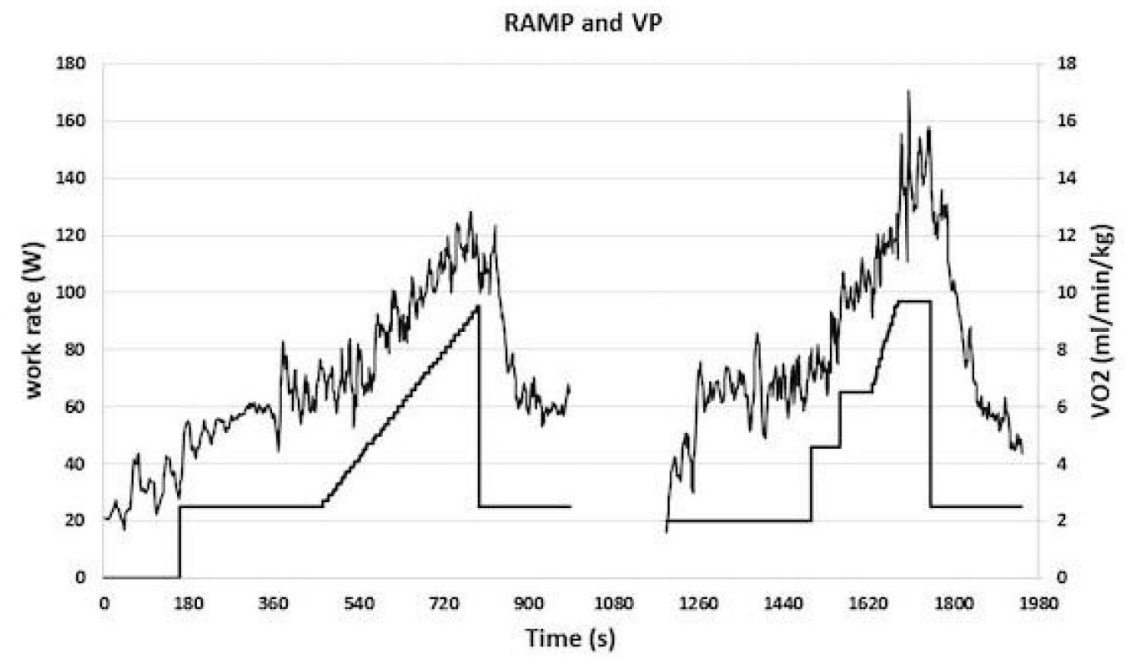

\section{Pulmonary Vascular Disease}

\section{P32}

\section{The Changing Epidemiology of Pulmonary Hypertension: Disease Characteristics and Clinical Outcome over Two Decades of the Swiss Registry}

\author{
P. Appenzeller ${ }^{1}$, M. Lichtblau ${ }^{1}$, C. Berlier ${ }^{1}$, J.-D. Aubert ${ }^{2}$, \\ A. Azzola ${ }^{3}$, J.-M. Fellrath ${ }^{4}$, T. Geiser ${ }^{5}$, F. Lador ${ }^{6}$, S. Pohle ', I. Opitz ${ }^{1}$, \\ M. Schwerzmann ${ }^{5}$, H. Stricker ${ }^{8}$, M. Tamm ${ }^{9}$, S. Saxer ${ }^{1}$, S. Ulrich ${ }^{1}$ \\ ${ }^{1}$ UniversitätsSpital Zürich, Zurich, ${ }^{2} \mathrm{CHUV}$, Lausanne, ${ }^{3} \mathrm{Cantonal}$ \\ Hospital Lucerne, Luzern, ${ }^{4}$ Hôpital Neuchâtelois Portalès, \\ Neuenburg, ${ }^{5}$ University Hospital Bern, Bern, ${ }^{6}$ University \\ Hospital of Geneva, Genf, ${ }^{7}$ Cantonal Hospital St. Gallen, \\ St. Gallen, ${ }^{8}$ Ospedale la Carità, Locarno, ${ }^{9}$ University Hospital \\ Basel, Basel, Switzerland
}

Introduction: This retrospective analysis of the Swiss Pulmonary Hypertension Registry provides long-term real-life data about epidemiology, demographics, disease characteristics and survival specially focusing on pulmonary arterial and chronic thromboembolic pulmonary hypertension (PAH and CTEPH).

Methods: Patients newly diagnosed with pulmonary hypertension (mostly PAH/CTEPH) registered from January 2001-June 2019 at 13 Swiss hospitals were reviewed and patient characteristics (age, BMI, gender, diagnosis), haemodynamics at baseline, treatment, days of follow-up and events (death, transplantation, pulmonary endarterectomy or loss to follow-up) at last visit were analysed. Patients were stratified into four time-periods according to their date of diagnosis. Survival was analysed overall and separately for PAH/CTEPH and time-periods.
Results: $1427 \mathrm{PH}$ patients were included (thereof $560 \mathrm{PAH}, 383$ $\mathrm{CTEPH})$. Over the years, age at baseline (mean \pm SD) significantly increased from $59 \pm 14$ years in 2001-2005 to 66 \pm 14 years in 2016$2019(\mathrm{p}<0.001)$ while the gender distribution tended towards equality. Mean pulmonary artery pressure and pulmonary vascular resistance significantly decreased over time (from $46 \pm 15$ to $41 \pm 11$ mmHg resp. $9 \pm 5$ to $7 \pm 4 \mathrm{WU}, \mathrm{p}<0.001)$. Three-year survival substantially increased over consecutive periods from $69 \%$ to $91 \%$ (for PAH 63\% to 95\%, for CTEPH $86 \%$ to $93 \%$ ) and was poorer in PAH than CTEPH independently of time period $(\mathrm{p}<0.001)$. Most patients were treated with mono- or combination therapy and an increasing number of CTEPH underwent pulmonary endarterectomy (40\% 2016-2019 vs. 15\% 2001-2005).

Conclusion: This long-term pulmonary hypertension registry reveals that over two decades of observation, newly diagnosed patients are older, less predominantly female, have less impaired haemodynamics and a better survival.

Disclosure: Ms. Appenzeller has nothing to disclose. Dr. Lichtblau reports personal fees from Boehringer-Ingelheim, personal fees from MSD, outside the submitted work. Dr. Berlier has nothing to disclose. Dr. Aubert has nothing to disclose. Dr. Azzola has nothing to disclose. Prof. Fellrath has nothing to disclose. Prof. Geiser has nothing to disclose. Dr. Lador reports personal fees from Actelion, personal fees from MSD, grants and personal fees from OrphaSwiss, outside the submitted work. Dr. Pohle has nothing to disclose. Prof. Opitz has nothing to disclose. Prof. Schwerzmann reports personal fees from Orpha Suisse, personal fees from MSD, personal fees from Actelion Janssen, outside the submitted work. Dr. Stricker has nothing to disclose. Prof. Tamm has nothing to disclose. Dr. Saxer has nothing to disclose. Prof. Ulrich reports grants and personal fees from Actelion SA, personal fees from MSD SA, grants and personal fees from Orpha Swiss, personal fees from Novartis SA, grants from Swiss National Science Foundation, grants from Zurich Lung, from null, outside the submitted work. 
P33

\section{Favorable Pregnancy Outcomes in Women with Predominantly Mild Pulmonary Arterial Hypertension}

N. Corbach ${ }^{1}$, C. Berlier ${ }^{1}$, M. Lichtblau' ${ }^{1}$, E.I. Schwarz ${ }^{1}$, F. Gautschi ${ }^{1}$, A. Groth ${ }^{1}$, R. Schüpbach ${ }^{2}$, F. Krähenmann ${ }^{3}$, S. Saxer ${ }^{1}$, S. Ulrich ${ }^{1}$

${ }^{1}$ Pneumologie, ${ }^{2}$ Anästhesiologie, ${ }^{3}$ Geburtshilfe,

UniversitätsSpital Zürich, Zürich, Switzerland

Introduction: Since pregnancy in women with pulmonary arterial hypertension (PAH) is associated with a high risk of morbidity and mortality, it is recommended that pregnancy should be avoided in PAH. However, some women with mild PAH may consider this recommendation as unsuitable. Knowledge on pregnancy outcomes and best management of PAH during pregnancy is limited.

Method: Data from all women with PAH who were followed during pregnancy by a multidisciplinary team at a tertiary referral center for PAH and who delivered between 2004 and 2020 were retrospectively analyzed in a case series. PAH risk factor profiles including New York Heart Association (NYHA) class, NT-proBNP, echocardiographic pulmonary arterial pressure (PAP) and right heart function were analyzed prior to, during and following pregnancy.

Results: In seven pregnancies of five women with PAH (median age $29(27 ; 31)$ years), there were no abortions or terminations. Five pregnancies were planned (all in NYHA I-II), two incidental (NYHA II, III). During pregnancy none of the women had complications or clinical worsening of PAH. After a median pregnancy duration of $371 / 7$ weeks all gave birth to healthy babies by cesarean section in spinal anesthesia. During pregnancy, PAP tended to increase, whilst the course of NYHA class and NT-pro-BNP were variable and no trend could be detected.

Conclusion: Women with PAH with a low risk profile closely followed by a multidisciplinary team had a favorable course during and after pregnancy, resulting in successful deliveries of healthy newborns via cesarean section.

Disclosure: Nothing to disclose

\section{P35 \\ Effect of Breathing Oxygen-Enriched Air on Exercise Performance in Patients with Pulmonary Hypertension in Heart Failure with Preserved Ejection Fraction}

J.Müller ${ }^{1}$, M. Lichtblau' ${ }^{1}$, S. Saxer ${ }^{1}$, A.F. Carta' ${ }^{1}$, S.R. Schneider ${ }^{1}$, C. Berlier ${ }^{1}$, M. Furian ${ }^{1}$, K.E. Bloch ${ }^{1,2,3}$, E.I. Schwarz ${ }^{1}$, S. Ulrich ${ }^{1,2,3}$

${ }^{1}$ Pulmonology, University Hospital Zurich, Zurich, ${ }^{2}$ Centre for Integrative Human Physiology, ${ }^{3}$ University of Zurich, Zürich, Switzerland

Introduction: To evaluate the effects of breathing oxygenenriched air (oxygen) on exercise performance in patients with pulmonary hypertension in heart failure with preserved ejection fraction (PH-HFpEF).

Methods: 10 patients with $\mathrm{PH}-\mathrm{HFpEF}$ (5 women, age $60 \pm 9 \mathrm{y}$, mPAP $37 \pm 14 \mathrm{mmHg}$, PAWP $18 \pm 2 \mathrm{mmHg}$, PVR $3 \pm 3 \mathrm{WU}$, resting $\mathrm{SpO}_{2} 98 \pm 2 \%$ ) performed two cycle incremental exercise tests (IET) and two constant work-rate exercise test (CWRET) at 75\% maximal work-rate $\left(\mathrm{W}_{\text {max }}\right)$, each with ambient air $\left(\mathrm{FiO}_{2} 0.21\right)$ and oxygen $\left(\mathrm{FiO}_{2} \mathrm{0.5}\right)$ in a randomized, single-blinded, cross-over design. The main outcomes were the change in $\mathrm{W}_{\max }$ (IET) and cycling-time (CWRET) with oxygen versus air. Blood gases at rest and endexercise, dyspnea by Borg 10 scale at end-exercise and continuous $\mathrm{SpO}_{2}$, minute ventilation (VE), carbon dioxide output $\left(\mathrm{VCO}_{2}\right)$, cerebral and quadriceps muscle tissue oxygenation (CTO and MTO) were measured.

Results: With oxygen vs. air, $\mathrm{W}_{\max }$ (IET) increased from $94 \pm 36$ to $99 \pm 36 \mathrm{Watt}$, mean difference $(95 \% \mathrm{CI}) 5.4$ ( 0.9 to 9.8 ), $\mathrm{p}=0.025$ and cycling-time (CWRET) from $532 \pm 203$ to $680 \pm 76$ seconds, +148 (31.8 to 264), $\mathrm{p}=0.018$. At end-exercise with oxygen, Borg dyspnea score, $\mathrm{VE} / \mathrm{VCO}_{2}$ were lower, whereas $\mathrm{PaO}_{2}$ and end-tidal $\mathrm{CO}_{2}$ were higher, other parameters were unchanged.

Conclusion: Patients with $\mathrm{PH}-\mathrm{HFpEF}$ not revealing resting hypoxemia, significantly improved their exercise performance with breathing oxygen-enriched air along with less dyspnea sensation, a better blood oxygenation and an enhanced ventilatory efficiency. PH-HFpEF patients may benefit from oxygen therapy during exercise.

Disclosure: Nothing to disclose

\section{P36 \\ Predictive Factors of Intra-Hospital Pulmonary Embolism Mortality}

A. Migaou, L. Eloued, L. Guetari, T. Lassoued, E. Mahmoudi,

N. Fahem, R. Kaddoussi, A. Ben Saad, S. Jobeur,

S. Cheikh Mhammed, N. Rouatbi

University of Medicine of Monastir, Monastir, Tunisia

Background: Pulmonary embolism (PE) is a common and potentially serious disease. It represents a public health problem 
due to its morbi-mortality. Its care concerns several specialities including pneumology. The aim of this work is to identify the factors influencing the intra-hospital PE mortality.

Methods: This is a descriptive and analytical retrospective study over a period of 20 years (from January 2000 to January 2020), including 94 patients who were hospitalized for PE.

Results: The average age was $58.14 \pm 16.14$ years. The sex ratio was 2.76 . The symptoms were dominated by dyspnea (48.94\%) and chest pain $(37.23 \%)$. The medical history was dominated by neoplasia (40.43\%) and COPD (23.4\%).

The most frequent factors favoring PE were neoplasia (40.43\%), bed rest (17.02\%) and recent surgery (7.45\%). Diagnostic confirmation was obtained by helical thoracic CT angiography in 78 cases $(82.98 \%)$, by pulmonary ventilation-perfusion scintigraphy in 9 patients $(9.57 \%)$ and by transthoracic ultrasonography in one case $(1.06 \%)$. Hemodynamic shock and acute respiratory failure complicated the evolution in $5.32 \%$ and $10.64 \%$ of cases respectively. Total intra-hospital mortality was $8.51 \%$.

In univariate analysis, the factors influencing intra-hospital mortality were history of COPD $(\mathrm{p}=0.01)$, history of lung cancer $((\mathrm{p}=0.043)$, chemotherapy $(\mathrm{p}=0.035)$, shock $(\mathrm{p}=0.002)$, tachycardia $>100 / \mathrm{min}(\mathrm{p}=0.004)$, polypnea $>20$ cycles $/ \mathrm{min}(\mathrm{p}=0.045)$, dyspnea $(p=0.025)$ and acute respiratory failure $(p=0.001)$. In multivariate analysis, acute respiratory failure was the only independent predictive factor of intra-hospital mortality. $(\mathrm{P}=0.001$; $\mathrm{OR}=2.33$; $\mathrm{CI}=[1.35-4]$ ).

Conclusion: PE is still a common but under-diagnosed pathology. Its mortality rate remains high despite improvements in diagnostic and therapeutic care.

Disclosure: Nothing to disclose

\section{P37 \\ Effect of a Daytrip to Altitude $(2500 \mathrm{~m})$ on Exercise Performance in Pulmonary Hypertension - A Randomized Controlled Trial}

\author{
S.R.Schneider $^{1,2}$, L.C. Mayer ${ }^{1}$, L. Mona ${ }^{1}$, B. Charlotte ${ }^{1}$, E.I. Schwarz ${ }^{1}$,

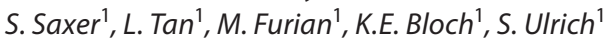 \\ ${ }^{1}$ Clinic of Pulmonology, UniversitätsSpital Zürich, Zürich, \\ ${ }^{2}$ Health Sciences and Medicine, Universität Luzern, Luzern, \\ Switzerland
}

Introduction: To investigate exercise performance and hypoxia-related health effects in patients with pulmonary hypertension $(\mathrm{PH})$ during a high-altitude sojourn.

Method: In a randomized crossover trial in patients with pulmonary arterial or chronic thromboembolic $\mathrm{PH}$ (PAH/ CTEPH) who were stable on therapy with resting $\mathrm{PaO}_{2} \geq 7.3 \mathrm{kPa}$, we compared symptom-limited constant work-rate exercise test (CWRET) cycling time during a daytrip to $2500 \mathrm{~m}$ vs. $470 \mathrm{~m}$. Further outcomes were symptoms, oxygenation and echocardiography. For safety, patients with sustained hypoxemia at altitude $\left(\mathrm{SpO}_{2}<80 \%\right.$ for $>30$ or $<75 \%$ for $>15 \mathrm{~min}$ ) received oxygen therapy. (ClinicalTrials.gov NCT03637153).
Results: 28 PAH/CTEPH (15/13)-patients, 13 females, mean \pm SD age $63 \pm 15 y$ were included. After $>3 \mathrm{~h}$ at $2500 \mathrm{~m}$ vs. $470 \mathrm{~m}$, CWRET-time was reduced to $17 \pm 11$ vs. $24 \pm 9 \mathrm{~min}$ (mean-difference $(95 \% \mathrm{CI})-6(-10$ to -3$)$ corresponding to $-27.6 \%(-41.1$ to -14.1) $\mathrm{p}<0.001$ but similar Borg-dyspnea scale. At altitude, $\mathrm{PaO}_{2}$ was significantly lower $(7.3 \pm 0.8$ vs. $10.4 \pm 1.5 \mathrm{kPa} ;-3.2(-3.6$ to -2.8$))$, whereas heart rate and tricuspid regurgitation pressure gradient (TRPG) were higher ( $86 \pm 18$ vs. $71 \pm 16 \mathrm{bpm} ; 15$ (7 to 23 ) and $56 \pm 25$ vs. $40 \pm 15 \mathrm{mmHg}$; (17(9 to 24$)$ ) and remained so until end-exercise, all $\mathrm{p}<0.001$. The TRPG/cardiac output slope during exercise was similar at both altitudes. Overall, $3 / 28(11 \%)$ patients received oxygen at $2500 \mathrm{~m}$ due to hypoxemia.

Conclusion: This randomized cross-over study showed that the majority of $\mathrm{PH}$-patients tolerate a daytrip to $2500 \mathrm{~m}$ well. At high vs. low altitude, the mean exercise time was reduced, albeit with a high inter-individual variability, and pulmonary artery pressure at rest and during exercise increased, but pressure-flow slope and dyspnea were unchanged.

Disclosure: Nothing to disclose
P38
Effect of Short-Term Oxygen Therapy on Exercise Performance in Patients with Cyanotic Congenital Heart Disease
S. Saxer ${ }^{1}$, L.-R. Calendo ${ }^{1,2}$, M. Lichtblau', J. Müller ${ }^{1}$, A.F. Carta', S. Ulrich ${ }^{1}$
${ }^{1}$ Pneumologie, UniversitätsSpital Zürich, Zürich, ${ }^{2}$ Zurich University of Applied Sciences, Winterthur, ${ }^{3}$ University Heart Center, UniversitätsSpital Zürich, Zürich, Switzerland F. Gautschi ${ }^{1}$, C. Berlier ${ }^{1}$, E.I. Schwarz ${ }^{1}$, K.E. Bloch ${ }^{1}$, M. Greutmann ${ }^{3}$,

Introduction: Patients with unrepaired cyanotic congenital heart disease (CHD) suffer from aggravated hypoxemia during exercise. We investigated the effect of supplemental oxygen on exercise performance in patients with cyanotic CHD.

Methods: In this randomized, sham-controlled, single-blind, cross-over trial cyanotic CHD-patients underwent four cycle exercise tests to exhaustion, while breathing either oxygen-enriched $\left(\mathrm{FiO}_{2} 0.50\right.$, oxygen $)$ or ambient air $\left(\mathrm{FiO}_{2} 0.21\right.$, air $)$ using incremental (IET) or constant work-rate (CWRET) protocols $(75 \%$ maximal work rate achieved under $\mathrm{FiO}_{2}$ 0.21). Pulmonary gasexchange, electrocardiogram, arterial blood gases, cerebral and quadriceps muscle tissue oxygenation (CTO and QMTO) by nearinfrared spectroscopy were measured.

Results: Seven patients with cyanotic CHD (4 Eisenmenger syndrome, 3 unrepaired cyanotic defects, 4 women) were included (median(quartiles) age $36(32 ; 50)$ years, BMI $23(20 ; 26) \mathrm{kg} / \mathrm{m}^{2}$ and $\mathrm{SpO}_{2}$ at rest $\left.87(83 ; 89) \%\right)$. When comparing supplemental oxygen with air during exercise, maximal work-rate in IET increased from $77(61 ; 114) \mathrm{W}$ to $83(67 ; 136) \mathrm{W}$, median difference $9(0 ; 22) \mathrm{W}$ $(\mathrm{p}=0.046)$ and CWRET-time increased from $412 \mathrm{~s}(325 ; 490)$ to $468 \mathrm{~s}(415 ; 553)$, median increase $56(39 ; 126) \mathrm{s}(\mathrm{p}=0.018)$. In both IET and CWRET, blood oxygenation was significantly higher and ventilatory equivalent for carbon dioxide significantly lower at 
end-exercise with oxygen compared to air, whereas CTO and QMTO did not significantly differ.

Conclusion: Patients with cyanotic CHD significantly improved their exercise performance, in terms of maximal workrate and endurance time, along with an improved blood oxygenation and ventilatory efficacy with supplemental oxygen compared to air. Patients with cyanotic CHD may benefit from oxygen therapy during physical activity and exercise.

Disclosure: Nothing to disclose

\section{P39 \\ Cardiovascular Disease and Chronic Obstructive Pulmonary Disease}

\section{S. Abdala, S. Aitbatahar, L. Amro}

Department of Pneumology, Mohamed VI University Hospital Center, Marrakesh, Morocco

Introduction: Cardiovascular diseases in chronic obstructive pulmonary disease (COPD) are frequent, dominated by chronic pulmonary heart disease, pulmonary hypertension and coronary artery disease. The aim of this study is to evaluate the prevalence of these complications in COPD and to describe the profile of patients with these conditions.

Patients and methods: Retrospective study spread over a 3-year period from January 2018 to December 2020 and including 114 cases hospitalized for acute exacerbation of COPD.

Results: Seventy-eight patients had cardiovascular complications, identified by clinical, ECG and echocardiography. The average age is 54 years, all patients are male, smoking is present in all our patients with an average of 57 pack years. The average number of exacerbations is 2 episodes per year. COPD is classified grade III in $25 \%$ and grade IV in $75 \%$. The ECG found right ventricular hypertrophy in $64 \%$ of cases, and right bundle branch block in $38 \%$ of cases. Echocardiography showed PH in 68 cases, right ventricular hypertrophy in 34 cases, coronary artery disease in 6 cases and congestive heart failure in 4 cases. The treatment was based on diuretics, antiarrhythmics, digitalis and anticoagulants, associated with the background treatment of COPD.

Conclusion: Through this study, we insist on the screening of cardiovascular complications in COPD whose most concerned are the elderly subjects, the non-compliant of the treatment and the frequent exacerbators.

Disclosure: Nothing to disclose

\section{Prevention (Tobacco/Environment)}

\author{
P40 \\ Impact of Smoking on Sleep Macro- \\ and Microstructure \\ M.K. Truong ${ }^{1}$, M. Berger ${ }^{2}$, J. Haba-Rubio ${ }^{2}$, F. Siclari ${ }^{2}$, \\ P. Marques-Vidal ${ }^{3}$, R. Heinzer ${ }^{1,2}$ \\ ${ }^{1}$ Service de Pneumologie, ${ }^{2}$ Centre d'Investigation et de \\ Recherche sur le Sommeil (CIRS), ${ }^{3}$ Service de Médecine Interne, \\ CHUV, Lausanne, Switzerland
}

Objectives: Existing data suggest that smoking may be associated with sleep disturbances. This study aimed to determine the association between smoking and both subjective and objective sleep quality.

Methods: Cross-sectional analysis of sleep characteristics in 3233 participants from the population-based CoLaus-HypnoLaus cohort ( $52.2 \%$ women, mean age $56.6 \pm 10.2$ years) who completed questionnaires on sleep quality, of whom 1489 (46\%) had a full polysomnography. Smoking data were self-reported; participants were classified by smoking status as current, former or never smokers. Primary outcomes were subjective sleep quality assessed by sleep questionnaires, and objective sleep quality based on polysomnography (sleep macrostructure), including power spectral analysis of the electroencephalogram (EEG) on C4 electrode (sleep microstructure), quantifying the relative amount of delta power (1-4 Hz), a marker of sleep depth, and alpha power $(8-12 \mathrm{~Hz})$, a marker or arousal.

Results: Current smokers had a shift toward faster sleep EEG activity with lower delta power in NREM (non-rapid eye movement) sleep compared with former and never smokers $(-2.8 \pm 0.4 \%$ and $-2.4 \pm 0.4 \%$, respectively; both $\mathrm{p}<0.001)$ and higher alpha power $(+0.8 \pm 0.2 \%$; $<<0.001)$ compared with never smokers. There was a dose-dependent negative association between EEG delta power and smoking intensity $\left(\mathrm{r}^{2}=-1.2[-1.9,-0.5] ; \mathrm{p}=0.001\right)$. Additionally, mean nocturnal oxygen saturation was lower in current smokers.

Conclusions: Current smokers had decreased objective sleep quality, with a dose-dependent association between smoking intensity and decrease in deep sleep-associated EEG delta power during NREM sleep, in addition to an increase in arousal-associated alpha power. Considering the importance of sleep quality for wellbeing and health, these results provide further data to support smoking cessation.

Disclosure: Nothing to disclose 


\section{P41 \\ Identification of Smoking Cessation Phenotypes for Individualized Counseling using the Persona Methodology}

\author{
M. Paciorkowski ${ }^{1}$, F. Baty ${ }^{1}$, S. Pohle ${ }^{1}$, E. Bürki ${ }^{1}$, M. Brutsche $e^{2}$ \\ ${ }^{1}$ Lung Center, ${ }^{2}$ Cantonal Hospital St. Gallen, St. Gallen, \\ Switzerland
}

Introduction: Smoking cessation is priority of public health. The rate of relapse in smokers attempting to quit is generally high and individualized smoking cessation counseling increases the probability of quitting success. The persona methodology aims to create hypothetical user archetypes allowing to better understand individual's needs, goals and behavior. The aim of the current study was to develop phenotypic personas of participants involved in smoking cessation program to support interventions and increase the chances of success during quitting attempts.

Material and methods: Data from 843 participants treated in the smoking cessation clinic of the Cantonal Hospital St. Gallen between March 2012 and December 2014 were extracted. Data included information about participant's longitudinal tobacco consumption together with demographics, smoking history and baseline dependence levels. Clusters among participants were identified using principal coordinates analysis combined with $\mathrm{k}$-means clustering. Clusters were interpreted with the help of inter-professional expert consensus and pertaining personas were created.

Results: Four main clusters of participants were identified, including long-term quitters, recursively try and fail, short-term returners and persistent smokers/reducers. Associated personas were created (Figure 1). The personas differed on various biopsychosocial domains including demographics, medical and psychological status, social and economic status and participant's preference regarding the intervention. Most participants should be able to identify and match with one of the four personas using baseline information together with basic measurements of motivation, self-confidence and nicotine dependence levels.

Conclusion: Meaningful clusters of participants attempting to quit smoking could be identified, and personas were successfully created to describe their main features. A persona-based approach could provide valuable tools to smoking cessation counselors by raising the awareness of the needs and demands of smokers to achieve long-term abstinence.

Disclosure: Nothing to disclose
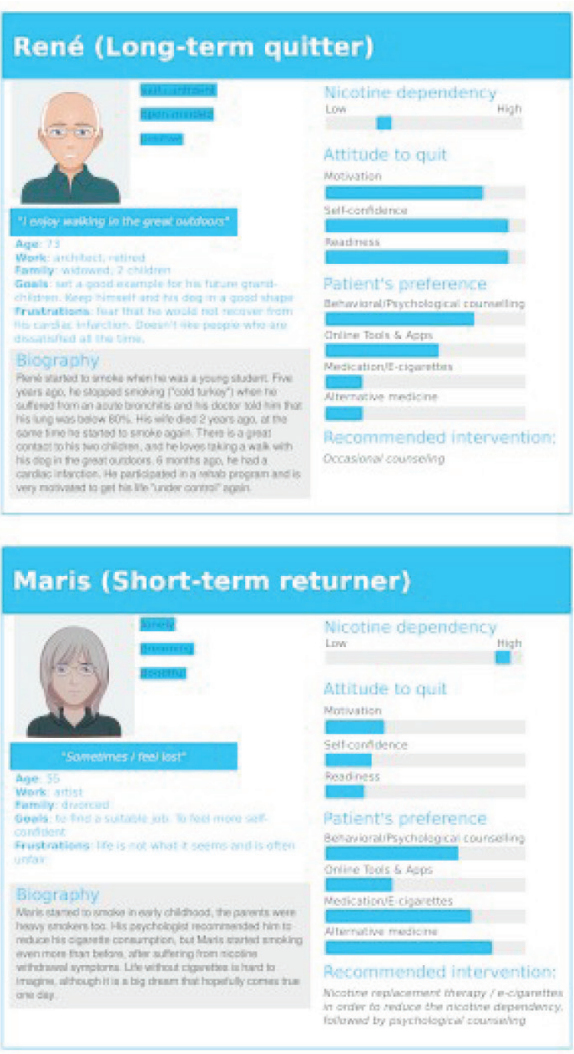
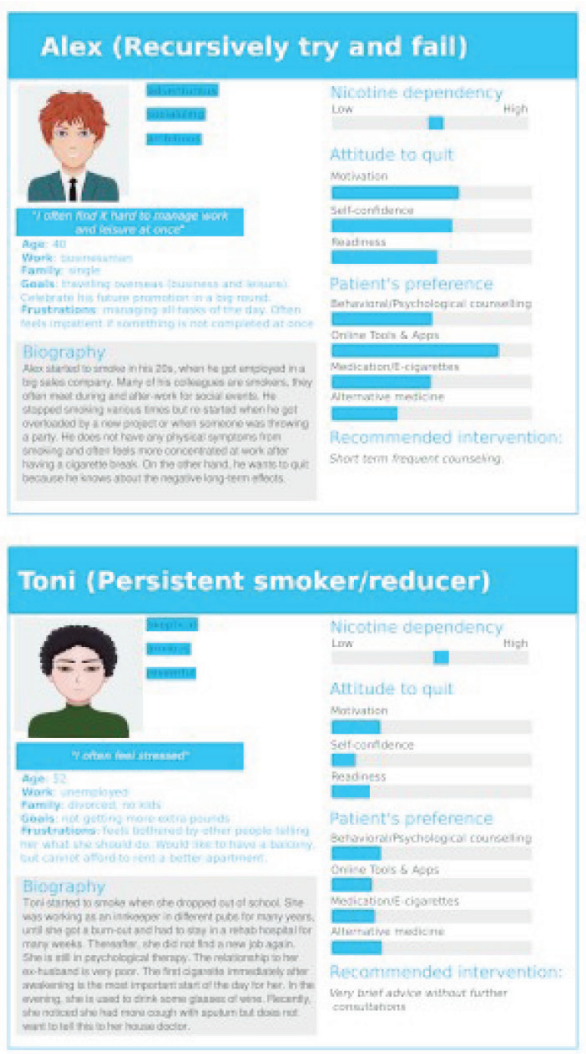

Fig. 1. 


\section{Sleep Disordered Breathing, Ventilation \& Oxygen}

\section{P42 \\ Acetazolamide for Preventing Acute Mountain Sickness in Healthy Older than $\mathbf{4 0}$ Years. RCT}

M. Furian $^{1,2}$, M. Mademilov ${ }^{2,3}$, A. Abdraeva ${ }^{3}$, M. Lichtblau ${ }^{1,2}$, U. Sheraliev $^{2,3}$, N.H. Marazhapov ${ }^{2,3}$, A. Buergin ${ }^{1}$, K. Bitos ${ }^{1}$, L. Mayer ${ }^{1}$, A. Reiser ${ }^{1}$, S.R. Schneider ${ }^{1,2}$, S. Buenzli ${ }^{1,2}$, S. Aydaralieva ${ }^{3}$, A. Muratbekova ${ }^{3}$, T.M. Sooronbaev ${ }^{2,3}$, S. Ulrich ${ }^{1,2}$, K.E. Bloch ${ }^{1,2}$

${ }^{1}$ Department of Respiratory Medicine, University Hospital Zurich, Zurich, Switzerland, ${ }^{2}$ Swiss-Kyrgyz High Altitude Medicine and Research Initiative, ${ }^{3}$ Pulmonary Department, National Center for Cardiology and Internal Medicine, Bishkek, Kyrgyzstan

Background: Acetazolamide is established for preventing acute mountain sickness (AMS) in young, healthy individuals. We evaluated whether acetazolamide prevents AMS in healthy persons older than 40y, an age group not included in previous studies.

Methods: In a randomized, placebo-controlled, double-blind parallel-design trial, 345 healthy lowlanders, mean \pm SD age $53 \pm 7 y$, $69 \%$ women, were treated with acetazolamide capsules $(375 \mathrm{mg} /$ day) or placebo, starting $24 \mathrm{~h}$ before ascent to and while staying 2 days at $3100 \mathrm{~m}$. Primary outcome: incidence of AMS, i.e., Lake Louise score $\geq 3$ including headache at $3100 \mathrm{~m}$. Secondary outcomes: incidence of other altitude-related illnesses, nocturnal pulse oximetry (mean $\mathrm{SpO}_{2}$ and number of $\mathrm{SpO}_{2}$ dips $>3 \% / \mathrm{h}$ as index of periodic breathing), and treatment-related side effects. (ClinicalTrials. gov NCT03561675).

Results: Fifty-four of 170 (32\%) participants receiving placebo and 38 of $175(22 \%)$ receiving acetazolamide experienced AMS (Chi-Square statistic $\mathrm{P}=0.035$ ), hazard ratio $0.48,95 \% \mathrm{CI}, 0.29$ to 0.80 , number needed to prevent one case of AMS 10 (95\%CI, 5 to 141). In participants using placebo, mean nocturnal $\mathrm{SpO}_{2}$ was lower $(84.6 \pm 0.2 \%)$ and $\mathrm{SpO}_{2}$ dips/h were more prevalent $(21.7 \pm 1.1)$ compared to participants using acetazolamide (mean difference $3.1 \%$ (2.5 to 3.7 ) and $10.5 / \mathrm{h}$ (8.4 to 12.7$), \mathrm{P}<0.001$, both comparisons. Other altitude-related illnesses occurred in 5(3\%) using placebo and $3(2 \%)$ using acetazolamide $(\mathrm{P}=0.497)$.

Conclusion: Among healthy lowlanders $\geq 40$ years staying at $3100 \mathrm{~m}$, the incidence of AMS was relatively low and further reduced by preventive treatment with acetazolamide. Acetazolamide improved nocturnal oxygenation and stabilized the breathing pattern.

Disclosure: Nothing to disclose

\section{P43 \\ Responsiveness of the $S^{3}$-NIV Questionnaire to Severe Episode of Respiratory Failure}

1. Guerreiro ${ }^{1}$, A. Schmit ${ }^{1}$, E. Schwarz ${ }^{2}$, P.M. Soccal ${ }^{1,3}$, J.-P. Janssens ${ }^{1,3}$, D. Adler ${ }^{1,3}$

'Division of Pulmonary Diseases, Geneva University Hospitals, Geneva, ${ }^{2}$ Department of Pulmonology and Sleep Disorders Centre, University Hospital Zurich, Zurich, ${ }^{3}$ Geneva Medical School, Geneva, Switzerland

Introduction: The $S^{3}$-NIV questionnaire was recently developed to provide clinicians with a simple and reliable tool to assess important domains such as respiratory symptoms, sleep quality and NIV-related side-effects in patients treated with home NIV.

Aim: To test the responsiveness of the $S^{3}$-NIV questionnaire to changes in health status during recovery from an acute episode of respiratory failure with hospital admission.

Methods: The $S^{3} \mathrm{NIV}$ questionnaire was administered at hospital admission and hospital discharge in consecutive patients admitted for an acute episode of respiratory failure.

Results: 19 patients ( 8 males, median age 64yrs (interquartile range [IQR]: 55-76), treated with home NIV for a median time of 24 months (IQR: 14-51) were enrolled at admission. 10 patients were diagnosed with COPD, 4 with neuromuscular disorders, 2 with restrictive disorders, 2 patients with obesity hypoventilation syndrome, and 1 with central breathing disturbances during sleep. Median length of stay was 14 days (IQR: 7-22). Mean S ${ }^{3}$-NIV questionnaire score at admission was 4.83 (SD 1.59, 95\% CI 4.07 to 5.60 ) and 6.08 (SD 1.77, 95\% CI 5.22 to 6.94) at discharge with a mean difference of 1.24 (SD 1.09, 95\% CI 0.71 to $1.77, \mathrm{p}<0.001$ ) (figure 1). Improvement of $\mathrm{S}^{3}$-NIV questionnaire score was more pronounced in COPD patients $(1.39,95 \% \mathrm{CI} 0.58$ to $2.19, \mathrm{p}=$ $0.004)$, compared to non-COPD patients $(1.08,95 \%$ CI 0.24 to 1.92 , $\mathrm{p}=0.02)$. Recovery from a severe exacerbation was associated with improvement in both the "respiratory symptoms" domain (1.61, $95 \% \mathrm{CI} 0.78$ to $2.43, \mathrm{p}<0.001)$ and in the "sleep and NIV-related side-effects" domain $(0.83,95 \%$ CI 0.24 to $1.43, \mathrm{p}=0.01)$ (figure 2 ).

Conclusion: Our observation suggests that the $\mathrm{S}^{3}$-NIV questionnaire is responsive to recovery from severe respiratory exacerbation. Further studies to estimate MCID of the $S^{3}$-NIV questionnaire using distribution and anchor-based methods are ongoing.

Disclosure: Nothing to disclose
SSP/SSTS - EFP

Joint Annual Conference 2021 
10

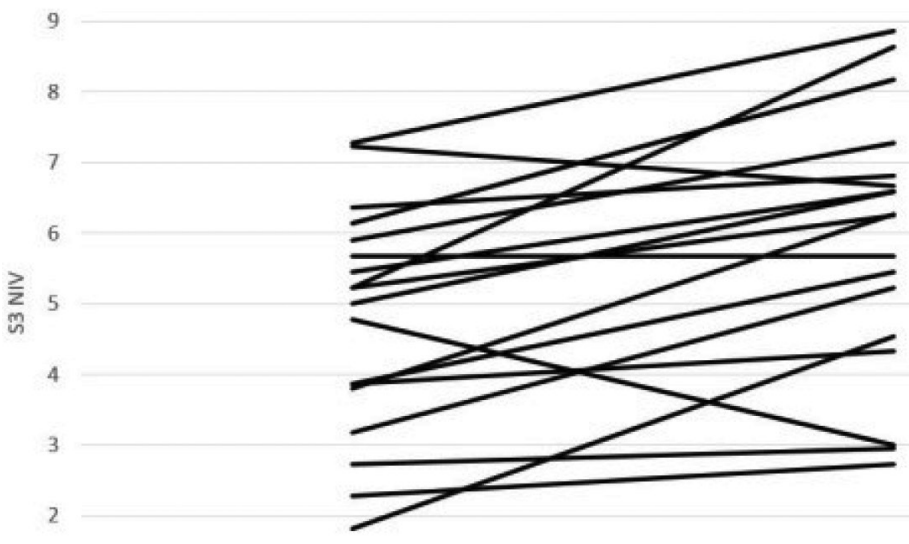

1

0

Fig. 1.

Q1) I suffer from breathing problems when I eat

Q4) I am often short of breath

05) I have trouble breathing when I speak

Q6) There is often mucus in my airways

Q7) I hawe difficulties breathing during physical exertion

Respiratory symptoms domain

Q2) 1 often have headache

Q3) I wake up at night with breathing difficulties

as) I am disturbed by leaks

Q9) My mask is uncomfortable

Q10) I recive too mudh air from my ventilator

Q11) I suffer from nasal or oral dryness

Sleep quality and NIV-related side-effects domain

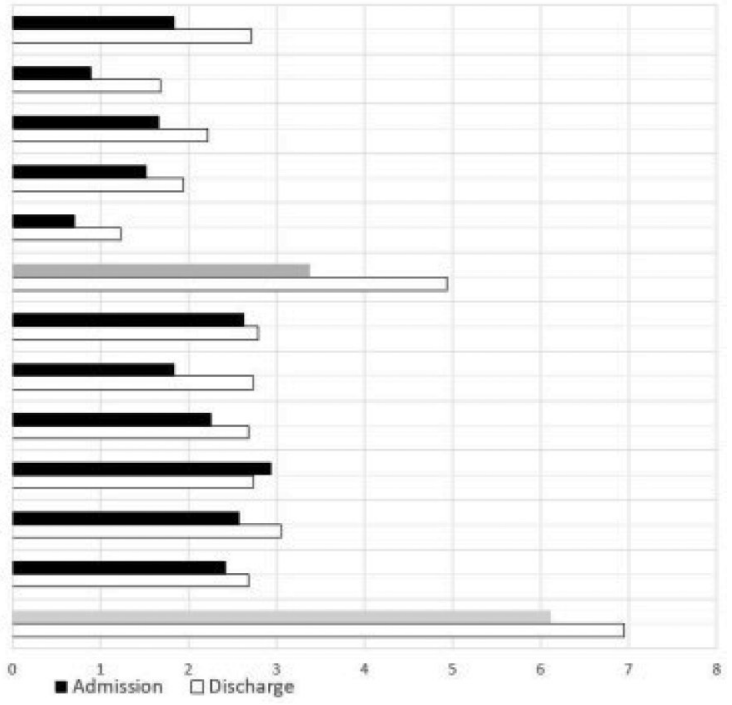

Fig. 2. 


\section{P44 \\ Variability of Obstructive Sleep Apnoea Severity Measures on Repeated Testing}

\author{
M. Meszaros ${ }^{1}$, M. Nido², S. Ulrich ${ }^{1}$, M. Kohler ${ }^{1}$, J. Steier ${ }^{2}$, \\ E.I.Schwarz
}

${ }^{1}$ Department of Pulmonology and Sleep Disorders Centre, University Hospital of Zurich, Zurich, Switzerland, ${ }^{2}$ Lane Fox Respiratory Unit and Sleep Disorders Centre, Guy's \& St Thomas' Hospital NHS Foundation Trust, London, United Kingdom

Introduction: Obstructive sleep apnoea (OSA) is defined and graded by thresholds of obstructive respiratory events and cyclic oxygen desaturations per hour while asleep. The aim of this study was to assess night-to-night variability of the oxygen desaturation index (ODI $\geq 4 \%$ dips) in a sleep clinic population.

Methods: Adults referred for OSA evaluation who underwent two consecutive nocturnal pulse oximetries were eligible. Based on the mean ODI over two nights, participants were grouped into "no" (< 5/h), "mild" (5-14.9/h), "moderate" (15-29.9/h), and "severe" (>30h) OSA. Outcomes were the change in ODI and coefficient of variation $(\mathrm{CV})$ between repeated studies in different OSA severity categories, and changes in ODI $\geq 10 / h, \geq 15 / h$, and in severity. Data are reported as mean $\pm \mathrm{SD}$.

Results: 623 of 900 included adults ( $58 \pm 13$ years) had OSA (ODI $21.8 \pm 21.4 / \mathrm{h}$ ). The change in ODI between nights was $6.7 \pm 7.6 / \mathrm{h}$ and increased with OSA severity (table 1 ). A CV of $27 \pm 3 \%$ indicated a high night-to-night-variability. Within the group of mild OSA, $27 \%$ changed between "no OSA" and "mild OSA". A change in ODI $\geq 10 / \mathrm{h}$ and $\geq 15 / \mathrm{h}$ was found in up to $43 \%$ and $29 \%$, respectively, and increased with severity as well. A severity category change was found in $35 \%$ with OSA and was most likely in moderate OSA.

Conclusion: The night-to-night variability in OSA severity, as measured by the ODI, is high and more than $1 / 3$ of patients changed severity class between two nights. Sleep studies recorded during a single night need to be interpreted with caution and in conjunction with symptoms as part of a comprehensive sleep evaluation to allow informed treatment recommendations.

Disclosure: Nothing to disclose

\section{P45 \\ Effect of Acetazolamide on Visuomotor Performance at Altitude in Healthy People Older than 40 Years: RCT}

\author{
A.E. Reiser ${ }^{1}$, M. Furian ${ }^{1,2}$, A. Buergin ${ }^{1}$, L. Mayer ${ }^{1}$, M. Lichtblau ${ }^{1,2}$, \\ S.R. Schneider ${ }^{1,2}$, P. Bader ${ }^{1}$, P. Appenzeller ${ }^{1}$, L. Muralt ${ }^{1}$, \\ M. Mademilov ${ }^{2,3}$, S. Aydaralieva ${ }^{3}$, A. Abdraeva $^{3}$, A. Muratbekova ${ }^{3}$, \\ T.M. Sooronbaev ${ }^{2,3}$, S. Ulrich ${ }^{1,2}$, K.E. Bloch ${ }^{1,2}$ \\ ${ }^{1}$ Department of Respiratory Medicine, University Hospital \\ Zurich, Zurich, Switzerland, ${ }^{2}$ Swiss-Kyrgyz High Altitude \\ Medicine and Research Initiative, ${ }^{3}$ Pulmonology, National \\ Center of Cardiology and Internal Medicine, Bishkek, Kyrgyzstan
}

Introduction: Hypoxia at high altitude may lead to cognitive impairment yet preventive therapy remains to be established, especially for middle-aged and older people. This study investigated whether acetazolamide prevents altitude-induced impairment of visuomotor performance in people older than 40 years.

Method: In this randomized placebo-controlled paralleldesign trial (NCT03536520), 59 healthy lowlanders aged 40-75y were assigned to acetazolamide $(375 \mathrm{mg} / \mathrm{d}, \mathrm{n}=34)$ or placebo $(\mathrm{n}=25)$, administered one day before ascent and while staying at high altitude $(3100 \mathrm{~m})$. Visuomotor performance was assessed at $760 \mathrm{~m}$ and $3100 \mathrm{~m}$ after arrival and in the next morning by a computer-assisted test (Motor Task Manager). It quantified deviation of a participant-controlled cursor affected by rotation during target tracking. Primary outcome was post-sleep recall of adaptation to rotation. Additionally, adaptation, immediate recall and correct test execution were evaluated.

Results: Compared to $760 \mathrm{~m}$, performance with placebo at $3100 \mathrm{~m}$ was worse during adaptation and immediate recall as mean deviation increased by $1.9^{\circ}(95 \%$ CI 0.3 to $3.5, p=0.024)$ and $1.1^{\circ}$ (0.4 to $1.8, \mathrm{p}=0.002)$. Post-sleep recall was unaffected $(\mathrm{p}=0.994)$ but correct test execution was $14 \%$ (9 to $19, \mathrm{p}<0.001$ ) less likely. Acetazolamide improved post-sleep recall by $5.6^{\circ}$ (2.6 to $8.6, \mathrm{p}<$ 0.001 ) and post-sleep probability of correct test execution by $36 \%$ (30 to $42, \mathrm{p}<0.001$ ) compared to placebo.

Conclusion: In healthy individuals older than 40 years, altitude impaired adaptation, immediate recall, and correct execution of a visuomotor task. Preventive acetazolamide treatment improved visuomotor performance after sleep at altitude and increased the probability of correct test execution.

Disclosure: Nothing to disclose

Table 1.

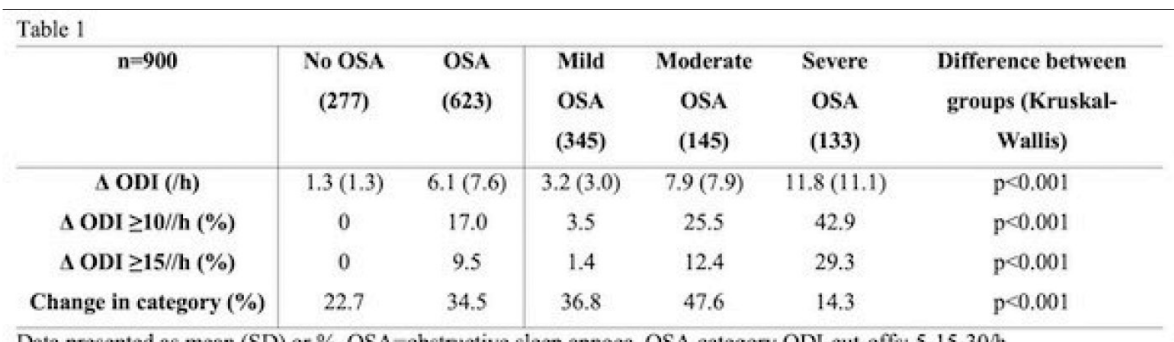

Data presented as mean (SD) or \%. OSA=obstructive sleep apnoea, OSA category ODI-cut-offs: 5-15-30/h 


\section{P46 \\ CPAP Improves Oxygen Saturation and Breathing Rate in COVID-19 Patients}

\author{
M. Schmiedeskamp ${ }^{1}$, M. Hofer ${ }^{1}$, S. Rezek ${ }^{2}$, I. Unger ${ }^{2}$, N. Ott ${ }^{2}$, \\ G. Volk ${ }^{2,3}$, J. Hetzel ${ }^{1,4}$ \\ ${ }^{1}$ Pneumologie, ${ }^{2}$ Institut für Therapien und Rehabilitation, \\ Kantonsspital Winterthur, ${ }^{3}$ Lunge Zürich, Winterthur, \\ Switzerland, ${ }^{4}$ Medizinische Onkologie und Pneumologie, \\ Universitätsklinikum Tübingen, Tübingen, Germany
}

Introduction: The most common cause of hospital admission in COVID-19 is hypoxic respiratory failure due to pneumonia. Oxygen administration via nasal cannula and high-flow nasal oxygen (HFNO) are used for treatment. So far, the use of CPAP for hypoxic failure due to bacterial or viral infections has only been established in particular situations such as concomitant immunosuppression. This is in contrast to several reports of beneficial effects of CPAP in COVID-19 patients. We established CPAP therapy with a specialised team (pulmonologists, respiratory therapists) on the COVID-19 ward. In the present study we present the effects on oxygen saturation ( $\mathrm{spO} 2)$ and respiratory rate.

Methods: Patients with respiratory insufficiency due to COVID-19 and pulmonary infiltrates on CT scan were treated with CPAP. Oxygen delivery via nasal cannula or HFNO was titrated to achieve a target spO2 between $92 \%$ and $95 \%$ prior to therapy. SpO2, respiratory rate and dyspnoea on the Borg scale (0-10) were measured before and 5 minutes after CPAP under identical settings. Results were analysed using the rank sum test for paired samples.

Results: 87 patients were included in the study (61 men, 26 women, mean age 63.5+/-14.6 y). 4C_MS was 9.6 (+/-3.9). The median duration of CPAP application was 60 minutes. Except in two patients, this was the initial treatment with CPAP for COVID19. $\mathrm{SpO} 2$ increased significantly by $1.3 \%(92.4+/-2.3 \%$ versus $93.7+/-1.7 \%, \mathrm{p}<0.001)$, respiratory rate decreased significantly by $2.1 /$ min $(24.2+/-6.0$ versus $22.0+/-5.1, \mathrm{p}<0.001)$. Patients experienced less breathlessness after CPAP therapy (Borg median 2.7 versus $1.9, \mathrm{p}<0.001$ ).

Conclusion: In the short term, CPAP therapy improves oxygen saturation and reduces respiratory rate and dyspnoea significantly in COVID-19 patients. For respiratory insufficiency in COVID-19 it can be applied safely on a general ward by a specialised respiratory team. Long term outcome, especially prevention of intubation, must be further evaluated.

Disclosure: Nothing to disclose

\section{P47 \\ Nocturnal Cerebral Oxygenation in Patients with COPD at Altitude - Effect of Acetazolamide}

Y.A. Schmuziger ${ }^{1}$, M. Mademilov ${ }^{2,3}$, A. Buergin ${ }^{1}$, P.M. Scheiwiller ${ }^{1,3}$, L. Mayer ${ }^{1}$, S.R. Schneider ${ }^{1,3}$, M. Lichtblau, ${ }^{1,3}$, K. Bitos ${ }^{1}$, L. Muralt', A. Groth' , U. Sheraliev 2,3 , N.H. Marazhapov 2,3 ${ }^{2}$ A. Akylbekov',3, G. Mirzalieva ${ }^{2,3}$, A. Oleinik ${ }^{2,3}$, T.M. Sooronbaev ${ }^{2,3}$, S. Ulrich ${ }^{1,3}$, K.E. Bloch ${ }^{1,3}$, M. Furian ${ }^{1,3}$

${ }^{1}$ Department of Respiratory Medicine, University Hospital of Zurich, Zurich, Switzerland, ${ }^{2}$ Department of Respiratory Medicine, National Center for Cardiology and Internal Medicine, Bishkek, ${ }^{3}$ Swiss-Kyrgyz High Altitude Medicine and Research Initiative, Zurich, Switzerland and Bishkek, Kyrgyzstan

Background: Patients with COPD may suffer from cerebral deoxygenation during altitude travel, especially during nights. Therefore, we quantified nocturnal cerebral and arterial oxygenation in lowlanders with COPD during a stay at high altitude and evaluated the effects of preventive acetazolamide therapy on these outcomes.

Methods: Patients with moderate to severe COPD $(40 \%<$ $\mathrm{FEV}_{1}<80 \%$ predicted), living $<800 \mathrm{~m}$, underwent nocturnal pulse oximetry $\left(\mathrm{SpO}_{2}\right)$ and cerebral tissue oximetry (CTO) by nearinfrared spectroscopy at $760 \mathrm{~m}$ and during the $1^{\text {st }}$ night at $3100 \mathrm{~m}$. Patients were randomized to $375 \mathrm{mg} /$ day acetazolamide or placebo starting $24 \mathrm{~h}$ before ascent and during the stay at $3100 \mathrm{~m}$. Effects of altitude and of acetazolamide on CTO and other outcomes were evaluated by regression analyses.

Results: 43 patients, 9 women, mean \pm SD age $55.4 \pm 8.9$ y, $\mathrm{FEV}_{1}$ $60 \pm 13 \%$ predicted, were included into the analysis. When ascending from 760 to $3100 \mathrm{~m}$ under placebo $(\mathrm{N}=17)$, nocturnal $\mathrm{SpO}_{2}$ and CTO decreased from $90.8 \pm 0.4 \%$ to $83.7 \pm 0.4 \%$ and from $66.5 \pm 1.0 \%$ to $63.4 \pm 1.0 \%$ ( $\mathrm{P}<0.05$ both comparisons); arterial (aODI) and cerebral (cODI) oxygen desaturation indices ( $>3 \%$ dips in $\mathrm{SpO}_{2}$ or CTO, respectively) increased by a mean $(95 \% \mathrm{CI})$ of $19.5 / \mathrm{h}$ (13.2 to $25.9)$ and $6.2 / \mathrm{h}$ (4.0 to 8.5). In patients assigned to acetazolamide $(\mathrm{N}=26)$, altitude effects were less pronounced compared to placebo: treatment effects(95\%CI) $\mathrm{SpO}_{2}+2.1 \%$ (2.1 to 2.2$)$, CTO +2.3\% (2.2 to 2.5$)$, aODI $-15.0 / \mathrm{h}$ (-23.1 to -6.9$)$, cODI $-4.4 / \mathrm{h}(-7.3$ to -1.5$), \mathrm{P}<$ 0.05 all effects.

Conclusion: Patients with COPD travelling to $3100 \mathrm{~m}$ experienced sustained and intermittent nocturnal cerebral deoxygenation associated with hypoxemia. Since acetazolamide improved oxygenation, it might mitigate adverse effects of altitude exposure.

Disclosure: Nothing to disclose 


\section{P48 \\ Time Course of Cerebral Oxygenation in Highlanders: 5-Year Prospective Study}

\author{
M.C. Luyken ${ }^{1,2}$, P. Appenzeller ${ }^{1,2}$, P.M. Scheiwiller ${ }^{1,2}$, M. Lichtblau ${ }^{1,2}$, \\ M. Mademilov 2,3 A. Muratbekova ${ }^{2,3}$, U. Sheraliev 2,3 A. Abdraeva $^{2,3}$, \\ N. Marazhapov ${ }^{2,3}$, T.M. Sooronbaev ${ }^{3}$, S. Ulrich ${ }^{1,2}$, K.E. Bloch $^{1,2}$, \\ M. Furian ${ }^{1,2}$ \\ ${ }^{1}$ Department of Respiratory Medicine, University Hospital \\ of Zurich, ${ }^{2}$ Swiss-Kyrgyz High Altitude Medicine and Research \\ Initiative, Zurich, Switzerland, ${ }^{3}$ National Center for Cardiology \\ and Internal Medicine, Bishkek, Kyrgyzstan
}

Background: Highlanders are exposed to chronic hypoxemia. We investigated whether this is associated with changes in cerebral oxygenation and cerebrovascular reactivity.

Methods: Highlanders, born and currently living above $2500 \mathrm{~m}$, participated in a prospective study in 2012 and 2017 (5y follow-up) carried out in a health post at $3250 \mathrm{~m}$ in Kyrgyzstan. Participants rested supine, breathing ambient air and oxygen $\left(\mathrm{FiO}_{2} 1.0\right)$ in random order, for $20 \mathrm{~min}$ each, followed by voluntary hyperventilation under ambient air and oxygen, respectively. Finger pulse oximetry $\left(\mathrm{SpO}_{2}\right)$, cerebral tissue oxygenation (CTO) and cerebral hemoglobin concentration $(\mathrm{cHb})$, as index of regional cerebral blood volume, were monitored by near-infrared spectroscopy, and arterial blood gases were obtained.

Results: 53 highlanders, aged $50 \pm 2 y$, participated. While breathing ambient air in 2017 vs. 2012, $\mathrm{PaO}_{2}$ was reduced, mean \pm SE, $7.40 \pm 0.13$ vs. $7.84 \pm 0.13 \mathrm{kPa}$; heart rate was increased $77 \pm 1$ bpm vs. $70 \pm 1$ ( $\mathrm{P}<0.05$ both changes $)$ but $\mathrm{CTO}$ remained unchanged, $67.2 \pm 0.7$ vs. $67.4 \pm 0.7 \%$. With oxygen breathing, $\mathrm{SpO}_{2}$ and CTO increased similarly in 2017 and 2012 by a mean $(95 \% \mathrm{CI})$ of $8.3 \%(7.5-9.1)$ vs. $8.5 \%(7.7-9.3)$, and $5.5 \%(4.1-7.0)$ vs. $4.5 \%(3.0-$ 6.0 ), respectively, $\mathrm{P}>0.05$. Hyperventilation resulted in less change in cHb in 2017 vs. 2012, mean difference $(95 \% \mathrm{CI})$ in change under ambient air 2.0 U/L(0.3-3.6); under oxygen, 2.1 U/L(0.5-3.7).

Conclusions: Over the course of 5 years, CTO in highlanders was preserved despite a decrease in $\mathrm{PaO}_{2}$. As this was associated with a mitigated response of cerebral blood volume to hyperventilation, adaptation of cerebrovascular reactivity might have occurred.

Disclosure: Nothing to disclose

\section{P49 \\ Comorbidities Associated with Sleep Apnea - A Nested Case-Control Study in Hospitalized Patients}

\author{
M. Moser ${ }^{1}$, F. Baty ${ }^{1}$, O.D. Schoch ${ }^{2}$, M. Brutsche ${ }^{1}$ \\ ${ }^{1}$ Lung Center, ${ }^{2}$ Cantonal Hospital St. Gallen, St. Gallen, \\ Switzerland
}

Introduction: Sleep apnea (SA) is a prevalent condition characterized by recurrent events of nocturnal apnea resulting in oxygen desaturations, activation of the vegetative nervous system and fragmented sleep. SA is typically associated with a range of comorbidities. The aim of the study was to clarify the prevalence and clusters of SA comorbidities using a nation-wide hospitalization database. The association between SA comorbidities, gender and motor vehicle accidents (MVA) was further investigated.

Material and methods: Data were extracted from a database provided by the Swiss Federal Office for Statistics, including all hospitalizations between 2002 and 2018. SA cases were defined by the ICD-10 codes G47.3*, including all the sub-codes. A control population was extracted matching SA cases for gender, age and month of hospitalization without a coded SA diagnosis. Clusters of comorbidities were identified using principal component analysis and their correlation with patient's demographics, prognosis (length-of-stay, in-hospital mortality) and risk of MVA was assessed.

Results: The number of hospitalization cases with a diagnosis of SA was 212 '581. The mean age was $70-74$ years and $73 \%$ were males. The most prevalent SA subtype was obstructive sleep apnea (50\%). SA patients had a median of 8 comorbidities (IQR: 5-11). Among a total of 10 '417 comorbidities, 64 were significantly overrepresented in SA compared to the matched population. The main over-represented comorbidities associated with SA (Figure 1) included blood and metabolic disorders, obesity, psychologic disorders, heart disease / hypertension, respiratory tract diseases and kidney diseases. Specific clusters of comorbidities differed among SA subtypes and genders. Hospitalizations involving SA in association with MVA were more frequent in younger obese patients. SA patients were not more hospitalized due to MVA compared to the controls.

Conclusion: Comorbidities are frequent in SA and mostly associated with worse prognosis. Comorbidities should be carefully assessed in the standard SA clinical check-up.

Disclosure: Nothing to disclose 


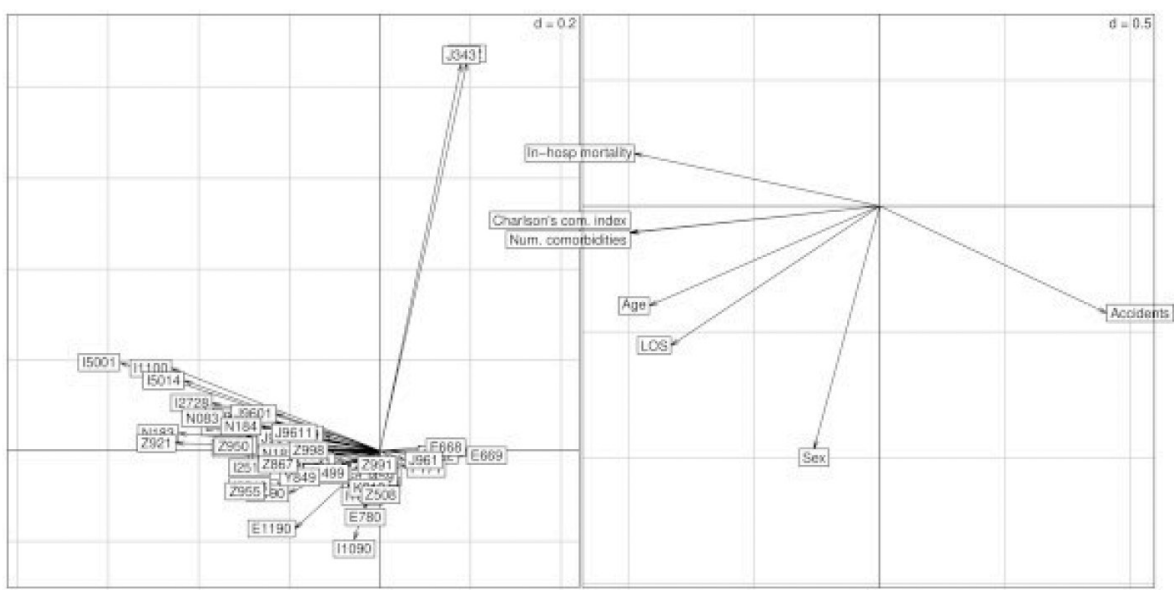

Fig. 1.

P50

Multi-Parametric Vital Sign Estimation for Monitoring of Sleep Disordered Breathing

F. Braun ${ }^{1}$, F. Baty ${ }^{2}$, J. Van Zaen ${ }^{1}$, G. Bonnier ${ }^{1}$, P. Renevey', P. Theurillat ${ }^{1}$, M. Proença' ${ }^{1}$, Y.-M. Proust ${ }^{1}$, M. Boesch ${ }^{2}$, O.D. Schoch ${ }^{2}$, D. Ferrario ${ }^{1}$, M. Lemay' ${ }^{1}$, S. Annaheim ${ }^{3}$, M. Brutsche ${ }^{2}$

${ }^{1}$ Centre Suisse d'Electronique et de Microtechnique (CSEM), Neuchâtel, ${ }^{2}$ Kantonsspital St. Gallen, ${ }^{3}$ Empa, St. Gallen, Switzerland

Introduction: Sleep apnea syndrome (SAS) is diagnosed by in-hospital polysomnography (PSG). PSG is considered the gold standard for SAS detection and characterization. However, PSG is relatively expensive and technically complex.

With the advent of smartwatches, optical heart rate monitoring via photoplethysmography (PPG) has become widely available. Being cost-efficient and unobtrusive, PPG would be a suitable technology for the early detection or long-term follow-up of SAS in ambulatory and home settings. We here investigated the feasibility of a novel wearable device to measure oxygen saturation $\left(\mathrm{SpO}_{2}\right)$, respiratory rate (RR) and cardiac interbeat intervals (IBI) at two body locations (wrist and upper arm).
Methods: Two proprietary PPG devices were placed at the wrist and upper arm of 9 patients with sleep disordered breathing (SDB; apnea-hypopnea index: $39.4 \pm 33.9$ events/h) undergoing overnight PSG recordings at the Cantonal Hospital St. Gallen. PSG reference measurements were compared to PPG-derived estimates of $\mathrm{SpO}_{2}, \mathrm{RR}$, and IBIs in terms of RMS error (for $\mathrm{SpO}_{2}$ ) and mean absolute error (for RR and IBI). For $\mathrm{SpO}_{2}$ and IBI, an algorithm automatically rejected erroneous estimates based on signal quality. $\mathrm{RR}$ was estimated in two ways, namely:

1) temporal modulation of IBIs (RRIBI), and

2) amplitude modulation of PPG signal (RRAmp).

Results: Table 1 lists the performance of $\mathrm{SpO}_{2}, \mathrm{IBI}, \mathrm{RR}_{\mathrm{IBI}}$ and $\mathrm{RR}_{\mathrm{Amp}}$ for wrist- and upper arm-based PPG devices compared to PSG. $\mathrm{SpO}_{2}$ and IBI errors are lower at the upper arm while for the $\mathrm{RR}$ no difference can be observed between the two body locations.

Conclusion: These preliminary results from a small group of SDB patients show promise for PPG devices as multi-parametric vital sign monitors. Future work should focus on algorithm development to combine multiple vital signs to a fully automated detection of apnea severity and to validate this approach in a larger group of patients.

Disclosure: Nothing to disclose

Table 1. Performance of PPG-derived vital sign estimation compared to PSG

\begin{tabular}{lcccccc}
\hline & \multicolumn{2}{c}{$\mathrm{SpO}_{2}$} & \multicolumn{2}{c}{ IBI } & $\mathbf{R R}_{\text {Amp }}$ & $\mathbf{R R}_{\text {III }}$ \\
& ARMs & Acceptance & MAE & Acceptance & MAE \\
$(\%)$ & $(\%)$ & $(\mathrm{ms})$ & $(\%)$ & $\begin{array}{c}\text { MAE } \\
(\mathrm{rpm})\end{array}$ \\
\hline Upper Arm & 1.8 & 81 & $8.0 \pm 6.1$ & 87 & $1.30 \pm 0.42$ & $1.67 \pm 0.85$ \\
Wrist & 2.5 & 75 & $11.5 \pm 11.0$ & 88 & $1.33 \pm 0.49$ & $1.68 \pm 0.81$ \\
\hline
\end{tabular}




\section{P51}

\section{Emerging Countries: Prevalence of Sleep Apnoea in Sub-Saharan Africa, the BeSAS Study}

\section{A.P. Wachinou ${ }^{1,2}$, A. Fiogbé $e^{2}$, S. Adè ${ }^{3}$, T. Totah ${ }^{4}$, F. Alovokpinhou ${ }^{2}$,} G. Agodokpessi ${ }^{2,5}$, D. Houinato ${ }^{4,5}$, R. Heinzer ${ }^{6}$

${ }^{1}$ Faculté des Sciences de la Santé, Université d'AbomeyCalavi, ${ }^{2}$ Centre National Hospitalier de Pneumophtisiologie de Cotonou, Cotonou, ${ }^{3}$ Faculté de Médecine, Université de Parakou, Parakou, ${ }^{4}$ Laboratoire d'Epidémiologie des Maladies Chroniques et Neurologiques, ${ }^{5}$ Université d'Abomey-Calavi, Cotonou, Benin, ${ }^{6}$ Centre d'Investigation et de Recherche sur le Sommeil, Lausanne, Switzerland

Introduction: Sleep apnoea is a health condition of growing concern worldwide but little is known about its prevalence in Africa. The aim of this study was to determine the prevalence of sleep apnoea and associated factors in general population in Benin, West Africa.

Method: BeSAS (Benin-Society-And-Sleep) is a crosssectional epidemiological study conducted from April 2018 to January 2021 aiming to compare sleep patterns and disorders in a population-based sample randomly selected from of a rural (Tanvè, Agbangnizoun city, central Benin) and an urban (3rd district of Cotonou) population in Benin. Berlin score was used to assess the risk of sleep apnea (high risk: $\geq 2$ ). Pittsburg Sleep Quality Index, Insomnia Severity Index and Epworth score were used to assess sleep quality, insomnia complaints and excessive daytime sleepiness. Factors associated with sleep apnea were assessed using logistic regression.

Results: A total of 2909 participants was recruited: 1201 (41.3\%, mean age $45.2 \pm 15.7$ y.o, $65.4 \%$ females) in the rural area and 1708 (58.7\%, mean age $44.4 \pm 13.5$ y.o, $58.3 \%$ females) in the urban area. After adjustment on age, sex and body mass index, the prevalence of high risk of sleep apnoea was 5.3\% [IC95\%: 4.1-6.7] in rural area vs 6.1\% [IC95\%:5.0-7.4] in urban area, $\mathrm{p}<0.001$. Factors associated with high risk of sleep apnoea were: age $\geq 40$ y.o (aOR: 2.6 [95\%CI:1.9-3.7], $\mathrm{p}<0.001$ ), male gender (aOR: 1.4 [95\%CI:1.1-1.9], $\mathrm{p}=0.019$ ), living in urban area (aOR: $1.8[1.3-2.5], \mathrm{p}<0.001$ ), excessive daytime sleepiness (aOR: 1.8 [95\%CI:1.4-2.5], $\mathrm{p}<0.001$ ), insomnia (aOR: 2.6 [1.8-3.5], $\mathrm{p}<0.001)$ and bad sleep quality (aOR: 1.8 [1.4-2.4], $\mathrm{p}<0.001)$.

Conclusion: Prevalence of sleep apnoea in general population seems low in Benin. Older age, male gender, living in urban area, excessive daytime sleepiness, insomnia and bad sleep quality are the associated factors. Ongoing analysis of polygraphic and actigraphic data will help to refine these findings.

Disclosure: Nothing to disclose

\section{P52 \\ Phenotyping Patients with COPD under Home Mechanical Ventilation: An Exploratory Study}

C. Cantero ${ }^{1}$, P. Pasquina ${ }^{1}$, C. Jaksic ${ }^{2}$, A. Poncet ${ }^{2}$, D. Adler ${ }^{1}$, M. Prella ${ }^{3}$, B. Egger ${ }^{4}$, C. Uldry ${ }^{4}$, A.B. Younossian ${ }^{5}$, P. Gasche ${ }^{1}$, J.-L. Pepin ${ }^{6}$, J.-P. Janssens ${ }^{1}$

${ }^{1}$ Department of Medicine, ${ }^{2}$ Department of Health and Community Medicine, Geneva University Hospital (HUG), Geneva, ${ }^{3}$ Division of Pulmonary Diseases, CHUV - Centre Hospitalier Universitaire, Lausanne, ${ }^{4}$ Division of Pulmonary Diseases and Pulmonary Rehabilitation Center, Hôpital de Rolle, Rolle, ${ }^{5}$ Centre Cardiopulmonaire, Hôpital de La Tour, Meyrin, Geneva, Switzerland, ${ }^{6}$ EFCR Laboratory, Thorax and Vessels, University Grenoble Alps, Grenoble, France

Introduction: Patients with COPD and chronic hypercapnic respiratory failure (CHRF) have been empirically treated by noninvasive ventilation (NIV) for decades, although randomized trials have yielded conflicting results. Level of hypercapnia and ventilator settings have been shown to be crucial. Phenotypes of COPD may also be of major importance.

Methods: In a cross-sectional observational study, we included all subjects under long term NIV in the Cantons of Geneva and Vaud (C Cantero et al, Chest 2020). In the present subpopulation analysis, we aimed to determine if, among the COPD population under long term NIV, a certain number of distinct phenotypes could be identified. We performed a Latent Class Analysis (LCA) of this population, using the following variables: age, gender, BMI, FEV1 (\% predicted), FVC (\% predicted), and relevant comorbidities (Table 1).

Results: Of the 486 patients included in the initial study, 190 (39\%) had COPD (55 of whom had an "Overlap syndrome"). Descriptive data are reported in Table 1. LCA led to the identification of 2 phenotypes (table 2$)$ : Type $1(n=108,57 \%)$ : obese subjects, with moderate airway obstruction, and a high prevalence of comorbidities (systemic hypertension, dyslipidemia, diabetes, vs Type $2(n=82,43 \%)$ : severe airway obstruction, with a normal BMI, more frequent pulmonary hypertension and mood disturbances. Age, gender, or use of NIV were similar in both groups. By logistic regression, 4 significant variables were identified (BMI, FEV1 and $\mathrm{FVC}$ as $\%$ predicted and diabetes): the equation generated classified correctly $90.5 \%$ of patients to their appropriate phenotype.

Conclusion: Among 190 COPD patients under long term NIV, 2 distinct phenotypes could be clearly identified. The logistic regression equation which was generated through this observation must be tested in other databases and should be evaluated as a possible prognostic marker in COPD with CHRF.

Disclosure: Nothing to disclose 


\begin{tabular}{|l|c|c|}
\hline & COpD & Overlap Syndrome \\
\hline Number of subjects & 135 & 55 \\
\hline Age (years) & $72(67 ; 78)$ & $71(65 ; 81)$ \\
\hline Gender (malo) & $68(50)$ & $36(65)$ \\
\hline Body mass Index (kg/m²) & $28(21 ; 33)$ & $32(28 ; 40)$ \\
\hline Time under Niv (months) & $23(9 ; 48)$ & $43(19 ; 73)$ \\
\hline Comorbidities & & \\
\hline Systemic hypertension & $91(67)$ & $46(84)$ \\
\hline Obesity & $52(38)$ & $38(69)$ \\
\hline Anxilety and/or depressive disorder & $82(17)$ & $16(29)$ \\
\hline Dyslipidemia & $56(41)$ & $28(51)$ \\
\hline Type II diabetes & $22(16)$ & $20(36)$ \\
\hline Gironic heart failure & $29(21)$ & $15(27)$ \\
\hline Pulmonary hypertension & $36(27)$ & $16(29)$ \\
\hline Cerebrovascular disease & $9(7)$ & $5(9)$ \\
\hline Treatment with oplates & $6(4.4)$ & $1(2)$ \\
\hline
\end{tabular}

Fig. 1. Baseline characteristics of study population.

\section{P53 \\ Experience with High-Flow Nasal Oxygen Therapy in COVID-19 Patients on a Regular Internal Medicine Ward}

M. Hofer ${ }^{1}$, M. Schmiedeskamp ${ }^{1}$, S. Rezek ${ }^{2}$, N. Ott ${ }^{1}$, I. Unger ${ }^{2}$, G. Volk ${ }^{2,3}$, J. Hetzel $^{1}$

${ }^{1}$ Pneumologie, ${ }^{2}$ Physiotherapie, Kantonspital Winterthur, Winterthur, ${ }^{3}$ Lunge Zürich, Zürich, Switzerland

Introduction / Objective: COVID-19 patients are at high risk for rapid pulmonary deterioration. Intubated patients might spend a long time in the intensive care unit with possibly poor outcome. High-flow nasal oxygen (HFNO) and CPAP are currently used alternative methods and may potentially prevent intubation. At the beginning of the second pandemic wave, we established a specialised team (pulmonologists, respiratory therapists) to support the COVID-19 unit (Cov19unit) affiliated to the normal internal medicine ward.

The aim of the study was to analyse the characteristics of HFNO-patients, with additional consideration of disease progression and outcome.

Methods: Retrospective analysis of all HFNO-patients on the Cov19unit between 10/2020 and 01/2021. The analysed parameters are summarised in the tables. As age is a risk factors in 4C-MS, an adapted score without age was calculated (4C-MSw/oAge). Three groups were analysed: Patients who required intubation, those discharged alive without intubation and those who died without intubation.

Table 1. Demogragics.

\begin{tabular}{|c|c|c|c|c|c|}
\hline & All & $\begin{array}{l}\text { Intubated } \\
\text { (patINTyes) }\end{array}$ & $\begin{array}{l}\text { Non-Intubated alive } \\
\text { (patINTno_alive) }\end{array}$ & $\begin{array}{l}\text { Non-Intubated died } \\
\text { (patINTno_died) }\end{array}$ & $\mathbf{p}$ \\
\hline N (\%) & 60 & $11(18.3)$ & $31(51.7)$ & $18(30.0)$ & \\
\hline $\begin{array}{l}\text { Gender: female / male } \\
{[\mathrm{N}(\%)]}\end{array}$ & $16 / 44(26.7 / 73.3)$ & $2 / 9(18.2 / 81.8)$ & $7 / 24(22.6 / 77.4)$ & $7 / 11(38.9 / 61.1)$ & $\mathrm{ns}^{* *}$ \\
\hline $\begin{array}{l}\text { Age }[\text { mean } \pm S D \\
\text { median }(1 \mathrm{st} / 3 \mathrm{st} \\
\text { quartile)] }\end{array}$ & $\begin{array}{l}72.4 \pm 12.8 ; 74.5 \\
(66.4 / 82.0)\end{array}$ & $\begin{array}{l}70.9 \pm 5.6 ; 70.0 \\
(68.2 / 74.8)\end{array}$ & $\begin{array}{l}67.7 \pm 14.4 ; 69.0 \\
(56.0 / 78.8)\end{array}$ & $\begin{array}{l}81.6 \pm 7.3 ; 83.0 \\
(78.8 / 85.3)\end{array}$ & $<0.0001^{\star * *}$ \\
\hline $\begin{array}{l}\text { 4C-MS* }[\text { mean } \pm \text { SD; } \\
\text { median }(1 \mathrm{st} / 3 \mathrm{st} \\
\text { quartile)] }\end{array}$ & $\begin{array}{l}12.4 \pm 3.5 ; 12.0 \\
(10.0 / 15.0)\end{array}$ & $\begin{array}{l}12.4 \pm 3.0 ; 13.0 \\
(12.0 / 13.8)\end{array}$ & $\begin{array}{l}11.3 \pm 3.5 ; 11.0 \\
(9.0 / 13.8)\end{array}$ & $\begin{array}{l}14.3 \pm 2.9 ; 14.0 \\
(12.0 / 16.0)\end{array}$ & $0.01^{\star * *}$ \\
\hline $\begin{array}{l}\text { 4C-MSw/oAge } \\
\text { [mean } \pm \text { SD; median } \\
\text { (1st/3st quartile)] }\end{array}$ & $\begin{array}{l}7.3 \pm 2.6 ; 8.0 \\
(5.4 / 9.0)\end{array}$ & $\begin{array}{l}7.5 \pm 2.0 ; 8.0 \\
(7.0 / 8.8)\end{array}$ & $\begin{array}{l}6.9 \pm 2.6 ; 7.0 \\
(5.0 / 8.8)\end{array}$ & $\begin{array}{l}7.9 \pm 3.0 ; 8.0 \\
(6.0 / 10.0)\end{array}$ & $\mathrm{ns}^{* * *}$ \\
\hline cPAP yes / no & $27 / 33(42.0 / 55.0)$ & $5 / 6(45.5 / 54.5)$ & $18 / 13(58.1 / 41.9)$ & $4 / 14(22.2 / 77.8)$ & 0.02 (alive vs death) ${ }^{* *}$ \\
\hline $\begin{array}{l}\text { Duration of } \\
\text { hospitalisation [mean } \\
\text { days } \pm \text { SD; median } \\
\text { (1st/3st quartile)] }\end{array}$ & $\begin{array}{l}14.9 \pm 8.9 ; 12.0 \\
(9.0 / 20.0)\end{array}$ & $\begin{array}{l}24.6 \pm 13.0 ; 24 \\
(12.4 / 35.8)\end{array}$ & $\begin{array}{l}15.0 \pm 6.5 ; 13 \\
(11.0 / 17.7)\end{array}$ & $\begin{array}{l}10.6 \pm 7.0 ; 8.5 \\
(4.0 / 19.1)\end{array}$ & $<0.001^{* * *}$ \\
\hline
\end{tabular}

*4C-MS: in-hospital mortality in patients admitted with COVID-19, based on age, gender, number of comorbidities, respiratory rate, peripheral oxygen saturation on room air, Glasgow Coma Scale, urea, C-reactive protein; ${ }^{* *}$ Fishers-Exact; ***Wilcoxon-Mann-Whitney. 
Table 2. HFNO parameters.

\begin{tabular}{|c|c|c|c|c|c|}
\hline & All & Intubated (patINTyes) & $\begin{array}{l}\text { Non-Intubated alive } \\
\text { (patINTno_alive) }\end{array}$ & $\begin{array}{l}\text { Non-Intubated died } \\
\text { (patINTno_died) }\end{array}$ & $\mathbf{p}$ \\
\hline $\begin{array}{l}\text { Start HFNO after admission } \\
\text { [mean days } \pm \text { SD; median } \\
\text { (1st/3st quartile })]\end{array}$ & $\begin{array}{l}3.0 \pm 3.1 ; 2.0 \\
(1.0 / 4.0)\end{array}$ & $2.7 \pm 1.5 ; 3.0(2.0 / 4.0)$ & $2.9 \pm 2.8 ; 2.0(1.0 / 5.0)$ & $3.4 \pm 4.3 ; 1.5(1.0 / 4.1)$ & $\mathrm{ns}^{*}$ \\
\hline $\begin{array}{l}\text { Duration of HFNO } \\
\text { [mean days } \pm S D ; \text { median } \\
\text { (1st/3st quartile)] }\end{array}$ & $\begin{array}{l}6.2 \pm 4.5 ; 5.0 \\
(3.0 / 8.0)\end{array}$ & $3.0 \pm 1.6 ; 3.0(2.0 / 3.0)$ & $6.6 \pm 2.8 ; 7.0(5.0 / 8.0)$ & $7.3 \pm 6.9 ; 4.0(3.0 / 8.1)$ & $0.03^{*}$ \\
\hline $\begin{array}{l}\text { pO2/FiO2 at start } \mathrm{HFNO} \\
{[\text { mean } \mathrm{mmHg} \pm \mathrm{SD} ; \text { median }} \\
\text { (1st/3st quartile)] }\end{array}$ & $\begin{array}{l}134 \pm 56 ; 131 \\
(94 / 163)\end{array}$ & $117 \pm 34 ; 106(94 / 133)$ & $141 \pm 53 ; 145(96 / 168)$ & $135 \pm 66 ; 120(83 / 163)$ & $\mathrm{ns}^{*}$ \\
\hline $\begin{array}{l}\text { Maximal flow on HFNO } \\
{[\text { mean litre/min } \pm \mathrm{SD} ;} \\
\text { median (1st/3st quartile)] }\end{array}$ & $\begin{array}{l}48 \pm 13 ; 50.0 \\
(40.0 / 60.0)\end{array}$ & $50 \pm 12 ; 53(48 / 60)$ & $46 \pm 14 ; 45(38 / 50)$ & $52 \pm 10 ; 60(46 / 60)$ & $\mathrm{ns}^{*}$ \\
\hline $\begin{array}{l}\text { Maximal FiO2 on HFNO } \\
{[\text { mean } \% \pm \text { SD; median }} \\
\text { (1st/3st quartile)] }\end{array}$ & $79 \pm 16 ; 79(69 / 94)$ & $85 \pm 12 ; 82(74 / 98)$ & $72 \pm 15 ; 73(62 / 83)$ & $89 \pm 14 ; 94(82 / 100)$ & $<0.001^{\star}$ \\
\hline $\begin{array}{l}\text { Time of maximal flow } \\
\text { and FiO2 after start HFNO } \\
\text { [mean hours } \pm \text { SD; median } \\
\text { (1st/3st quartile)] }\end{array}$ & $\begin{array}{l}34 \pm 42 ; 24.0(0 / 48) \\
44 \pm 84 ; 24(8 / 36)\end{array}$ & $\begin{array}{l}85 \pm 12 ; 82(74 / 98) \\
33 \pm 36 ; 28(0 / 48)\end{array}$ & $31 \pm 46 ; 24(0 / 43)$ & $40 \pm 38 ; 24(12 / 68)$ & $\mathrm{ns}^{*}$ \\
\hline $\begin{array}{l}\text { Duration of maximal flow } \\
\text { and FiO2 [mean } \\
\text { hours } \pm \mathrm{SD} \text {; median (1st/3st } \\
\text { quartile)] }\end{array}$ & $44 \pm 84 ; 24(8 / 36)$ & $9 \pm 8 ; 7(2 / 9)$ & $25 \pm 24 ; 24(8 / 26)$ & $102 \pm 143 ; 36(24 / 132)$ & $<0.01^{\star}$ \\
\hline
\end{tabular}

${ }^{\star}$ Wilcoxon-Mann-Whitney.

Results: From 10/2020 to 01/2021, more than 500 patients have been hospitalised in the Cov19unit. Characteristics of the 60 HFNO-patients are shown in table 1, parameters of HFNO treatment in table 2 .

Of the $29(48 \%)$ patients who did not want to be intubated, 11 (38\%) were discharged alive. There was one possible treatmentassociated COVID-19 infection of a respiratory therapist and no serious adverse events during HFNO.

Conclusions: HFNO therapy can be safely delivered in a normal ward with the support of a specialist respiratory team. More than half of HFNO-patients survived without intubation. Pulmonary deterioration leading to intubation or death was higher with age, but did not correlate with 4C-MSw/oAge. patINTno alive were hospitalised for a shorter time compared to intubated patients, but longer compared to all other non-HFNO-treated patients ( $15.0 \pm 6.5$ vs $7.9 \pm 7.2$, data not shown). Importantly, when patINTyes deteriorated, they did so rapidly, wherefore careful monitoring must be ensured.

Disclosure: Nothing to disclose

\section{P54 \\ Sleep-Disordered Breathing Ventilation and Oxygen Limits Concentration and Causes Daytime Sleepless, Non Restorative Sleep, Cognitive Deficits, Change in Mood, Morning Headaches, Dry Mouth, Impotence}

\section{Akujinwa}

Imo State University (IMSU), Owerri, Nigeria

The term sleep disordered breathing ventilation and oxygen. Encompasses a range of conditions characterised by abnormal breathing during sleep; in many cases this is associated with narrowing or obstruction of the upper airway (pharynx). The disordered breathing ranges from intermittent, partial obstruction of the airway without sleep disturbance (snoring) to, at the other end of the spectrum, frequent apnoeas associated with repetitive hypoxaemia and arousals leading to sleep disruption and daytime sleepiness. The term 'obstructive sleep apnoea' (OSA) refers to intermittent obstruction of the airway, irrespective of the presence of daytime symptoms. If symptoms result, the condition is called obstructive sleep apnoea syndrome (OSAS), also known as obstructive sleep apnoea/hypopnoea syndrome (OSAHS). 
The following are 7 sleep disordered breathing ventilation and oxygen.

1. Loud snoring/snorting: It is often associated with a sleep disorder called obstructive sleep apnoea (OSA) which is accompanied by witnessed breathing pauses during sleep, excessive daytime sleepiness, difficulty concentrating, morning headaches, Sore throat upon awakening.

Restless sleep. Gasping or choking at night. High blood pressure and Chest pain at night.

2. Witnessed apnoeas by bed partner.

3. Awakening with choking.

4. Nocturnal restlessness.

5. Vivid, strange or threatening dreams.

6. Diaphoresis (sweating).

7. Hyper salivation, teeth grinding prevention:

Therefore, primary care physicians as well as physicians in a variety of specialties need to be aware of OSAS and sleep disordered breathing in order for the problem to be diagnosed and treated as promptly as possible. Secondary prevention therefore includes screening patients with the above conditions for symptoms potentially related to (OSAS). Once (OSAS) is diagnosed, its treatment is relatively straightforward. Lifestyle measures, such as weight loss, alcohol consumption and smoking should be addressed. However, the commonest and most rapidly effective treatment for moderate-to-severe (OSAS) is with nocturnal continuous positive airway pressure (CPAP) (figure 2). This is usually delivered through the upper airway using a mask over the nose.

Disclosure: Sleeping disordered breathing ventilation and oxygen includes. 1. Central sleep apnoea (CSA), in which periodic cessation of breathing occurs without obstruction of the airway and which, in adults, is seen mainly in heart failure 2. Obesity hypoventilation syndrome (OHS) in which breathing is reduced throughout sleep, with or without accompanying narrowing or obstruction of the upper airway. Nocturnal symptoms of OSAS are generally reported by a bed partner. The most common are snoring (which is almost always a feature), snorting, choking attacks terminating a snore, and witnessed apnoeas. Apnoeic episodes are reported by about $75 \%$ of bed partners. A number of clinical features are associated with OSAS. (Table 2), but the predictive value of any single one for diagnosis is limited and not all will co-exist in the same patient. History and clinical examination alone (including blood pressure and BMI) can predict the presence of OSAS in only $50 \%$ of patients attending a sleep disorders clinic: definitive diagnosis requires overnight investigation. Obesity (particularly central, BMI $>30 \mathrm{~kg} \cdot \mathrm{m}-2$ ) Large neck circumference $(>40 \mathrm{~cm})$ Small mandible, small maxilla Retrognathia (back-set jaw) Dental malocclusion, overbite Reduced nasal patency High and narrow hard palate Elongated and low-lying uvula Enlarged tonsils and adenoids.

\section{Thoracic Interventions \& Oncology, Thoracic Surgery, Lung Transplantation}

P55
Accelerated Treatment Concept
for Postpneumonectomy Empyema

G.-M. Monsch, I. Inci, S. Hillinger, C. Caviezel, O. Lauk, I.Opitz,

D. Schneiter

Department of Thoracic Surgery, University Hospital Zurich, Zurich, Switzerland

Introduction: Postpneumonectomy empyema remains a clinical challenge. We analysed the clinical outcome in patients undergoing our accelerated treatment concept avoiding open chest treatment in a retrospective cohort study.

Method: We treated 58 patients with postpneumonectomy empyema from November 2005 to July 2020. The therapy consisted of repeated open or minimal-invasive surgical debridement of the pleural cavity, negative pressure wound therapy and definitive closure after installation of antibiotic solution plus systemic antimicrobial therapy. If present, bronchopleural fistula was closed and reinforced using omentum and/or a muscle flap.

Results: Out of 58 patients ( 44 men, 14 women; median age, 65 years; range, 18-79), 56 (97\%) were treated with an open surgical procedure, 2 (3\%) with a minimal-invasive. Postpneumonectomy empyema was present on the right side in 33 patients (16 with bronchopleural fistula), in 25 patients on the left ( 3 with bronchopleural fistula). Median time between pneumonectomy and postpneumonectomy empyema was 2 months (range, 0-182). Bronchopleural fistula was treated by different surgical methods (omentum, 13; omentum combined with muscle flap, 2; muscleflap, 4). Until definitive closure, two re-interventions in median (range, 1-8 (open procedure); 0-3 (minimal-invasive)) were necessary. The chest was definitely closed in all patients, in median within 8 days (range, 2-40 (open procedure); 0-7 (minimal-invasive)). Median hospitalization time was 20 days (range, 8-132 (open procedure); 8-39 (minimal-invasive)). In-hospital mortality was $12.1 \%$ (7 patients). Postpneumonectomy empyema was successfully treated in $100 \%$ of survivors, including 7 (12.1\%) patients with recurrence-empyema.

Conclusion: With this accelerated empyema treatment concept, open chest windows can be avoided, and patients are effectively and safely cured from postpneumonectomy empyema maintaining integrity of the chest wall.

Disclosure: Isabelle Opitz has the following disclosures: AstraZeneca: Advisory Board; Medtronic: Institutional Grant; Roche: Speakers fee and Institutional Grant for Fellowship. 


\section{P56 \\ Surgery of Remnant Lung after Contralateral Pneumonectomy is Safe}

K. Furrer, E. Iliev, W. Weder, S. Hillinger, D. Schneiter, I. Inci, I. Opitz

Department of Thoracic Surgery, University Hospital Zurich, Zurich, Switzerland

Introduction: Surgery of the residual lung after contralateral pneumonectomy for progressing NSCLC is challenging because of high risk of the second procedure and uncertain long-term results. We aimed to assess the safety of surgery in this patientgroup.

Methods: Retrospective chart review was carried out on patients undergoing surgery of the remnant lung after contralateral pneumonectomy between 2001-2020. 10 right- and leftsided anatomical- and 10 extended pneumonectomies were performed, R0 rate(17/20, 85\%). 13 NSCLC ( 9 adenocarcinomas, 4 squamous cell carcinomas) and 7 other tumors were diagnosed.

Results: Median age was $61(20-77)$ years, $55 \%$ females. The resection of the contralateral lung (18 wedge resections, 1 chest wall resection, 1 partial pleurectomy) was performed 18.5 months later (0-142) with 30 - and 90 day $5 \%(1 / 20)$ and $10 \%(2 / 20)$ mortality. The morbidity after second surgery in $10 / 20$ patients was predominantly caused by grade I minor (7/20) complications. Major complications including death of one patient due to asystole by sick sinus syndrome and one patient reoperated by hematothorax due to misdosed oral anticoagulation. Histology revealed 12 metastases, 4 secondary primary, 2 benign lesions and 2 patients with a synchronous- respective metachronous tumors. The median overall survival (OS) and progression-free survival (PFS) were 37 [95\% CI: 20, 53.9] and 13 [95\% CI 0, 38.1] months, respectively. The time interval between surgeries: more 12 months versus less than 12 months ( $9 / 14$ vs 5/6 deaths) and complete (R0) resection of the primary tumor versus R1/R2 resection belonged to the factors influenced OS and PFS (figure).

Conclusions: The present analysis reveals that lung resection in the remaining lung after pneumonectomy can be performed in selected patients with acceptable morbidity and mortality. The prognosis is poor for patients with incomplete resection for the primary tumor and for those treated by second surgery earlier than 12 months after the pneumonectomy.
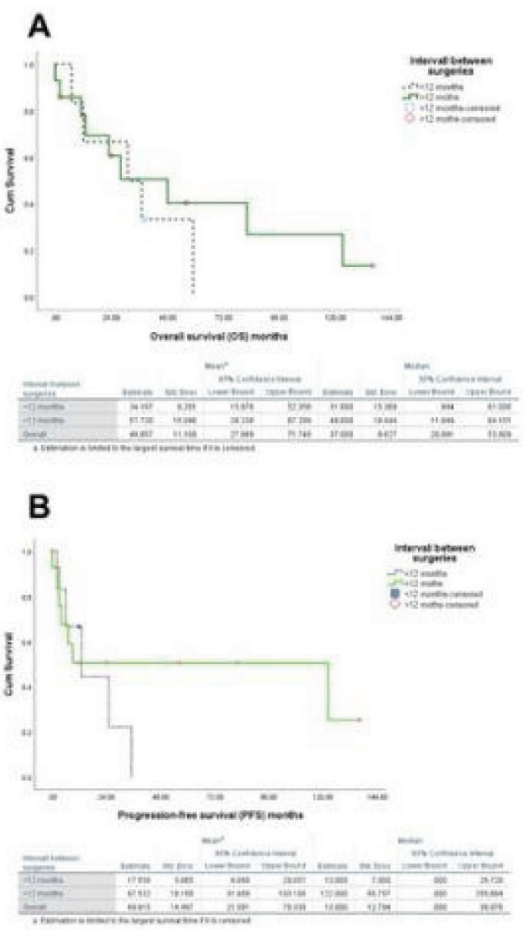
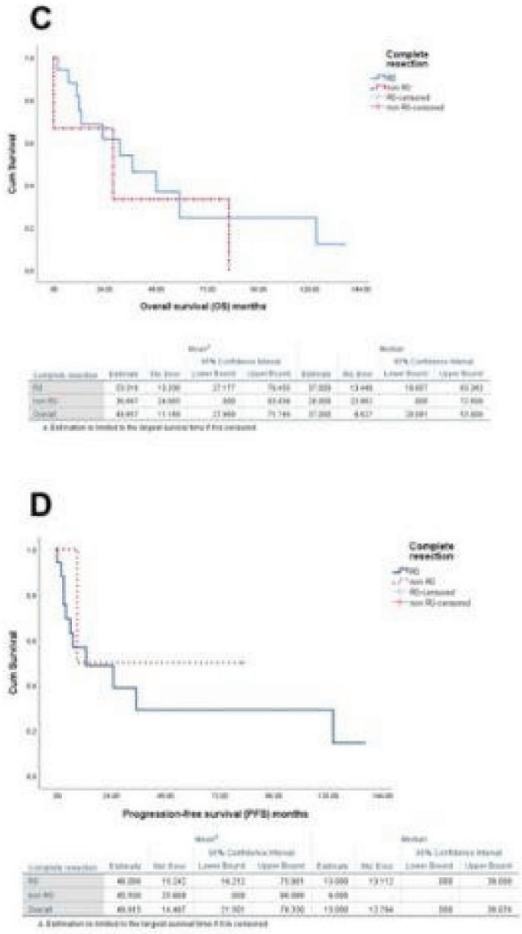

Fig. 1. Overall- and progression free survival.

\section{OS and PFS according to interval between surgeries more than 12 months vs less than 12 months (A, B) and according to Ro vs non $\mathrm{RO}$ - resection of the primary tumor $(C, D)$.}


Disclosure: Isabelle Opitz has the following disclosures: AstraZeneca: Advisory Board; Medtronic: Institutional Grant; Roche: Speakers fee and Institutional Grant for Fellowship.

\section{P57 \\ Cytokine Analysis in Serum and Broncho-Alveolar Lavage Fluid of Patients with immune-Checkpoint Inhibitor Associated Pneumonitis}

\author{
B. Kowalski' , P.R. Bezel', A. Valaperti ', U.C. Steiner ${ }^{1}$, D. Scholtze ${ }^{2}$, \\ S. Wieser ${ }^{3}$ D. Franzen ${ }^{1}$ \\ ${ }^{1}$ University Hospital Zurich, ${ }^{2}$ Stadtspital Triemli, ${ }^{3}$ Stadtspital \\ Waid, Zurich, Switzerland
}

Introduction: Immune-checkpoint inhibitors (ICI) are emerging drugs for treatment of several malignancies by activating T-lymphocytes attacking antigen-presenting cells such as those of a tumor. However, due their cytotoxic potential, immune-related adverse events, including ICI-associated pneumonitis (ICIaP) must be considered. Diagnosis thereof is often challenging, and its pathogenesis has not yet been fully understood. The aim of study was to investigate cytokines in serum and bronchoalveolar lavage fluid (BALF) expressed in patients with ICIaP compared to healthy individuals and to further two control groups including patients with other inflammatory response to lung injury as seen in lung cancer, sarcoidosis or ILD.

Method: This study is a sub-investigation of a recently established serum and BALF biobank (BALOTHEK). The following 11 cytokines were simultaneously analyzed in BALF and serum of each study participant: interferon gamma, tumor necrosis factor alpha, interleukin (IL) 1b, IL-2, IL-4, IL-5, IL-6, IL-8, IL-12p70, IL-13 and IL-17A. Enrolled patients were retrospectively clustered in four groups according to clinical and radiological presentation, confirmed by histology: ICIaP, healthy control, lung cancer, and sarcoidosis/ILD.

Results: From January 2018 until June 2019, 12 patients with ICIaP (after treatment with Pembrolizumab, Ipilimumab, combination thereof, or Durvalumab) and 12 healthy subjects as matched control group were included in the first analysis. Of the investigated cytokines, only IL- 6 was significantly increased in BALF but not in serum of patients with ICIaP ( $\mathrm{p}=0.044$, adjusted for multiple comparisons). Also compared to matched comparators with lung cancer $(n=12)$ or sarcoidosis/ILD $(n=12)$, IL-6 remained significantly increased in patients with ICIaP $(\mathrm{p}=0.005)$.

Conclusion: Cytokine profile assessed in BALF shows promising potential for diagnosis. IL-6 may not only contribute to better understanding of pathophysiology but also herald therapeutic implications for anti-IL-6 directed drug treatment (Tocilizumab).

Disclosure: Nothing to disclose

\section{P58 \\ Salvage Surgery in Patients with Locally Advanced Non-Small Cell Lung Cancer - Outcomes and Longtime Results}

\section{K. Furrer, R. Werner, S. Hillinger, D. Schneiter, I. Inci, I. Opitz}

Department of Thoracic Surgery, University Hospital Zurich, Zurich, Switzerland

Objective: In patients with stage IIIB or stage IV non-small cell lung cancer (NSCLC), current guidelines recommend a combination of systemic treatment including chemotherapy, targeted therapy or immunotherapy with definitive radiotherapy. However, relapses still occur in $>30 \%$ of all patients within 2 years. In a selected group of patients with local tumor recurrence or residual disease, so-called "salvage resection" can be performed with curative intent. Since previous reports on this approach are scarce and candidate selection remains challenging, we aimed to assess the outcomes of salvage surgery.

Methods: We retrospectively identified patients with initial stage IIIB or stage IV NSCLC who underwent salvage anatomical lung resection in curative intent to treat relapse or residual disease after definitive systemic treatment and/or radiotherapy between 2001-2020. Patients were only included if surgical resection had not been part of the first line treatment approach.

Results: Among 30 patients (53\% male, mean age 60.5 years) patients, 7 had initial stage IIIB and 23 had stage IV NSCLC. Anatomical resections included 21 lobectomies/bilobectomies with 8 extended resections, 1 segmentectomy and 8 extended pneumonectomies. R0 resection was achieved in $93.3 \%$ and pathological complete response was seen in 7 cases. Morbidity rate after surgery was $36.7 \%(11 / 30)$ with 5/30 minor (Grade II) and 7/30 major (Grade IIIA-V) complications. 30- and 90-day mortality was $0 \%$ and $10 \%$. Median overall survival (OS) and progressionfree survival (PFS) were 5 years [95\% CI: 2.83; 7.16] and 12 months [95\% CI: 0; 27] respectively. R0 resection of the primary tumor was an independent factor influencing OS and PFS (69 months [95\% CI $24.9 ; 113, p=0.24$ ] and 22 months [ $95 \%$ CI $1.45 ; 42.5, p=0.002]$ ) respectively.

Conclusion: In selected patients with stage IIIB or stage IV NSCLC, radical resection for relapse or residual disease after definitive systemic treatment and/or radiotherapy showed promising OS and PFS.

Disclosure: Isabelle Opitz has the following disclosures: AstraZeneca: Advisory Board; Medtronic: Institutional Grant; Roche: Speakers fee and Institutional Grant for Fellowship 
Fig. 1. Overall- and progression free survival.
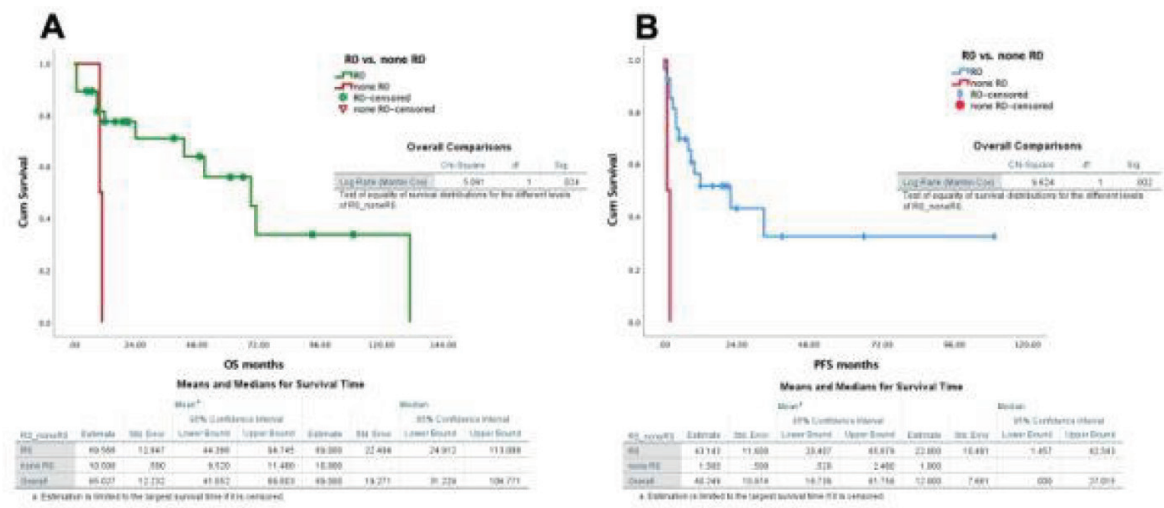

\section{P59 \\ Extended Resections for Advanced Stages T3/T4 NSCLC including N2 Disease after Neoadjuvant Treatment: SAKK Pooled Analysis $(16 / 96,16 / 00,16 / 01)$}

\begin{abstract}
K. Furrer ${ }^{1}$, W. Weder ${ }^{1}$, D. Betticher ${ }^{2}$, M. Pless ${ }^{3}$, R. Stupp ${ }^{4}$, T. Krueger ${ }^{5}$, J. Perentes ${ }^{5}$, R.A. Schmid ${ }^{6}$, D. Lardinois ${ }^{7}$, M. Furrer ${ }^{8}$, M. Frueh ${ }^{9}$, S. Peters ${ }^{5}$, A. Curioni-Fontecedro ${ }^{10}$, R.A. Stahel ${ }^{10}$, S. Rothschild ${ }^{7}$, E.I. Eboulet ${ }^{11}$, S. Hayoz ${ }^{11}$, S. Thierstein ${ }^{11}$, C. Biaggi ${ }^{11}$, I. Opitz

${ }^{1}$ Department of Thoracic Surgery, University Hospital Zurich, Zurich, ${ }^{2}$ Cantonal Hospital Freiburg, Freiburg, ${ }^{3}$ Cantonal Hospital Winterthur, Winterthur, Switzerland, ${ }^{4}$ Northwestern University Feinberg School of Medicine, Chicago, IL, United States, ${ }^{5}$ University Hospital Lausanne, Lausanne, ${ }^{6}$ University Hospital Bern, Bern, ${ }^{7}$ University Hospital Basel, Basel, ${ }^{8}$ Cantonal Hospital Graubuenden, Chur, ${ }^{9}$ Cantonal Hospital St. Gallen, St. Gallen, ${ }^{10}$ Department for Medical Oncology and Hematology, University Hospital Zurich, Zurich, ${ }^{11}$ Swiss Group for Clinical Cancer Reserach (SAKK) Coordinating Center, Bern, Switzerland
\end{abstract}

Introduction: Surgical treatment of patients with T3/4 locally advanced NSCLC including single or multilevel N2 remains a matter of debate across countries. Several trials have demonstrated that selected patients benefit from surgery, when R0-resection can be achieved. We aimed to assess resectability and outcome of resections for locally advanced T3/T4 tumors after induction treatment in a pooled analysis of three prospective multicenter trials.

Methods: Analyses included patient data of 197 out of 368 stage III patients with T3/T4 NSCLC enrolled in phase III/II SAKK trials, who were treated with induction chemo-or chemoradiotherapy followed by resection. Resectability, safety, long-term outcomes are reported, according to $8^{\text {th }}$ TNM classification.

Results: Median age was 60 years, $67 \%$ males. 38/197 patients were not considered for resection for medical (80\%) or technical (20\%) reasons. 159 anatomical lung resections (36 extended resections) and systematic lymphadenectomy were performed with $79 \%$ (127/159) R0 rate (30/36 R0 extended resections). Overall 30and 90 day mortality for T3/T4 were 3\%(5/159) and 7\%(11/159), respectively, with no significant difference for extended resections versus all other $(\mathrm{p}=0.077, \mathrm{p}=0.126)$. Morbidity was $32 \%$ with $70 \%(36 / 51)$ minor complications. 3-, 5-, 10-year overall survival (OS) after extended resections for T3/T4 stages (any R-status) were 61\% [95\%CI: 43-75\%], 44\% [95\%CI: 27-59\%], 29.5\% [95\%CI: 13-48\%] (figure), respectively. Pretreatment N2 (single, double, multiple in 177/197 patients) involvement had no impact on OS (T3: $\mathrm{p}=0.09$ for, $\mathrm{T} 4: \mathrm{p}=0.86$ ). Multivariate analysis revealed factors associated with improved overall survival: age (HR 1.027 [95\%CI: $1.004,1.05$ ], $\mathrm{p}=0.02$ ) and $\mathrm{R} 0$ resection (HR 0.448 [95\%CI: 0.287 , $0.700], \mathrm{p}<0.001)$.

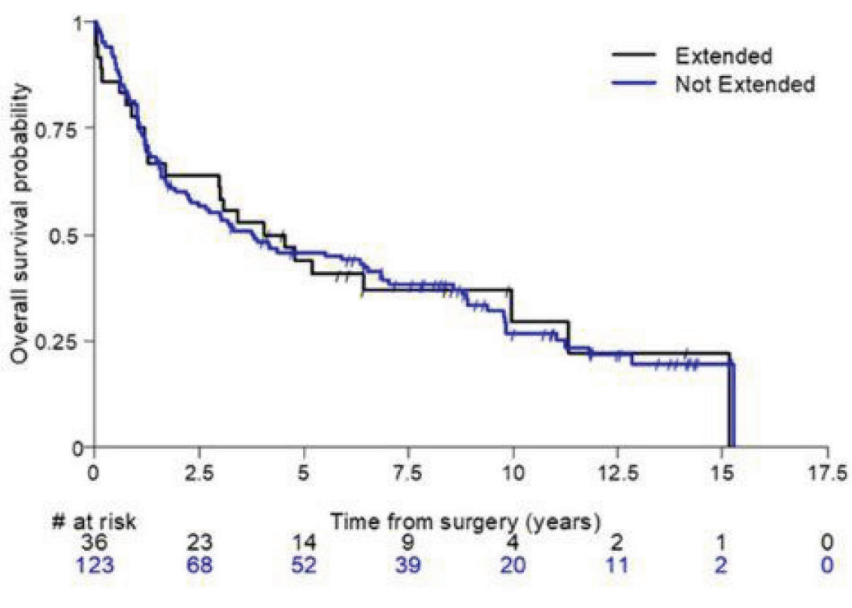

\begin{tabular}{cccc}
\hline \multicolumn{4}{c}{ Pulmonary resection, $N=159$} \\
Year & $\%[95 \% \mathrm{Cl}]$ & Not Extended, $\mathrm{N}=123$ & \\
\hline 3 & $61.1 \%[43.3 \%, 74.8 \%]$ & $54.2 \%[45.0 \%, 62.6 \%]$ & 0.472 \\
5 & $43.7 \%[27.2 \%, 59.2 \%]$ & $45.7 \%[36.7 \%, 54.3 \%]$ & 0.834 \\
10 & $29.5 \%[13.1 \%, 48.1 \%]$ & $26.8 \%[18.4 \%, 35.8 \%]$ & 0.789 \\
\hline
\end{tabular}

Comparison of OS between extended or not extended resections (any $R$ ) for patients with locally advanced T3/T4 (8th edition) III stage NSCLC

Fig. 1. Comparison of OS between extended or not extended resections. 
Conclusions: Surgery for advanced resectable T3/T4 NSCLC stage including single and multiple N2 disease prior to induction resulted in 79\%-R0 resection rate and only 7\% 90-day mortality in this challenging clinical situation. Favorable long-term overall survival was independent from the pre-induction $\mathrm{N}$ status and the extent of resection.

Disclosure: Isabelle Opitz has the following disclosures: AstraZeneca: Advisory Board; Medtronic: Institutional Grant; Roche: Speakers fee and Institutional Grant for Fellowship Walter Weder has the following disclosures: Advisory Board (AstraZeneca), Speaker (AstraZeneca, Covidien/Medtronic), Teaching Grant (Covidien/Medtronic) Miklos Pless has the following disclosures: Advisory Board (Abbvie, AstraZeneca, Boehringer Ingelheim, BMS, Eisei, MSD, Novartis, Pfizer, Roche, TAkeda, Merck), Travel Grant (AstraZeneca, Boehringer Ingelheim, BMS, Vifor), Speaker's fee (Janssen)

\section{P60 \\ Lung Ultrasound Compared to Chest X-ray for Exclusion of Pneumothorax after Interventional Bronchoscopies}

\section{Mangold, C. Steinack, S. Grimaldi, F. Rüber, D. Franzen}

UniversitätsSpital Zürich, Zürich, Switzerland

Introduction: Routine chest X-ray (CXR) is recommended to exclude pneumothorax (PTX) after transbronchial bronchoscopic procedures. However, concerns regarding radiation exposure, expenses, staff requirements and infrastructure must be considered. Lung ultrasound (LUS) is a promising imaging technology to detect post-interventional PTX. However, there are only a few studies on this topic yet. This retrospective study aims to investigate the diagnostic yield of LUS compared to CXR for exclusion of PTX after bronchoscopies with increased PTX risk.

Method: From September 2019 until August 2020, patients were screened for PTX using LUS followed by CXR within two hours after bronchoscopies for transbronchial biopsy (forceps, brush and cryoprobe) or endobronchial valve placement.

Results: Totally, 296 patients were included (median age 63 years, $42.9 \%$ female). The leading indication for bronchoscopy was suspected pulmonary malignant lesion (39.9\%) and the most frequent intervention was transbronchial forceps biopsy $(74.3 \%)$. The median time between the end of the procedure and LUS and CXR was $3.5 \mathrm{~min}$ and $63 \mathrm{~min}$, respectively. There was a PTX rate of $2.4 \%$ (7/296). Sensitivity, specificity, positive predictive value and negative predictive value of LUS for diagnosis of PTX were $57.14 \%$ (95\% CI: 18.41-90.10\%), 98.96\% (95\% CI: 97.00-99.79\%), 57.14\% (95\% CI: 26.74-82.97\%), and 98.96\% (95\% CI: 97.59-99.56\%), respectively. Those of CXR were 100\% (95\% CI: 59.04-100\%), 98.96\% (95\% CI: 97.00-99.79\%), 70.0\% (95\% CI: 43.08-87.79\%), and $100 \%$, respectively. The diagnostic yield to detect a PTX was comparable between LUS and CXR ( $\mathrm{p}=0.25)$ using McNemar's test.

Conclusion: LUS seems to be a promising alternative to CXR for exclusion of PTX after interventional bronchoscopies with a comparable specificity and negative predictive value. However, the time between the end of the procedure and PTX screening has likely a relevant effect on diagnostic yield. A prospective study considering this variable and with higher sample size is needed.

Disclosure: Nothing to disclose

\section{P61 \\ 3D-Quantitated Lung Perfusion SPECT/CT: Impact on Intended Management Compared to Lung Perfusion Scan in Marginal Candidates for Lung Resection Surgery}

\author{
M. Mallaev ${ }^{1}$, A.-F. Chirindel' ${ }^{2}$, M. Wiese ${ }^{1}$, A. Hojski ${ }^{1}$, M. Tamm ${ }^{3}$, \\ G. Nicolas ${ }^{2}$, D. Lardinois ${ }^{1}$, D. Stolz ${ }^{3}$ \\ ${ }^{1}$ Clinic of Thoracic Surgery, ${ }^{2}$ Clinic of Radiology and Nuclear \\ Medicine, ${ }^{3}$ Clinic of Pulmonology, University Hospital Basel, \\ Basel, Switzerland
}

Introduction: Based on previous studies, single-photon emission computed tomography/computed tomography (SPECT/CT) has been proven to be more accurate and reproducible than planar lung perfusion scintigraphy. We conducted a study to evaluate the impact of 3D-quantitated lung perfusion SPECT/CT on intended management in candidates for lung resection surgery.

Methods: Retrospective study including candidates for lung resection surgery with lung perfusion imaging. Patients underwent preoperative evaluation according to ERS/ESTS algorithm. The lobar contribution to the total lung perfusion was estimated using planar antero-posterior, posterior oblique and three-dimensional anatomical SPECT/CT method (CT Pulmo 3D and XSPECTQuant, Siemens). The difference in lobar perfusion with resulting changes in the extent of lung resection were analyzed to reveal possible changes in operability.

Results: 120 patients (46 females) with known lung cancer or pulmonary lesion considered for resection with available lung perfusion scintigraphy and SPECT/CT were enrolled. The mean age $( \pm S D)$ of patients was $68 \pm 9$ years, the target lesions were located in upper lobe in $57.7 \%$ and in lower lobe in $33.5 \%$. The median [IQR] $\mathrm{FEV}_{1}$ was 70.5\% [52-84] and median DLCO 56.6\% [47.1-67.4]. The planar posterior oblique method, compared to 3D-quantitated SPECT/CT, underestimated the perfusion of upper lobes by a median difference of $5 \%$ (right [2-9; IQR]; left [2.5-8], $\mathrm{p}=<0.0001)$, while it overestimated the both lower lobes (left by $4 \%$ [2-7]; right by $6 \%$ [2-9], $\mathrm{p}=<0.0001$ ). In contrast to the planar scintigraphy based evaluation, $4(3.3 \%)$ patients with upper lobe lesions were classified as inoperable when $3 \mathrm{D}$-quantitated SPECT/CT was used for calculation of predicted postoperative lung function.

Conclusion: In selected patients with upper lobe lesions, 3D-quantitated SPECT/CT would have changed the treatment strategy from operable to inoperable. Importantly, post-operative mortality in this particular subgroup was disproportionally high. 3D-quantitated SPECT/CT shall be further evaluated as it might improve preoperative risk stratification in marginal lung resection candidates.

Disclosure: Nothing to disclose 


\section{P63}

\section{The Choice of Priming Solution for Cardiopulmonary Bypass during Pulmonary Endarterectomy for CTEPH Patients has Important Impact}

K. Furrer', D. Bettex ${ }^{2}$, T. Horisberger ${ }^{2}$, I. Inci', O. Schmid ${ }^{3}$, N.G. Nagaraj ${ }^{3}$, H.T. Morselli', B. Battilana', R. Schuepbach ${ }^{4}$,

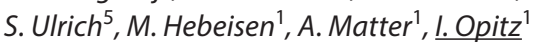

${ }^{1}$ Department of Thoracic Surgery, ${ }^{2}$ Institute of Anaesthesiology, ${ }^{3}$ Clinical Perfusionist at the University Heart Center, ${ }^{4}$ Institute of Intensive Care, ${ }^{5}$ Department of Pulmonology, University Hospital Zurich, Zurich, Switzerland

Purpose: Pulmonary endarterectomy (PEA) is the treatment of choice for chronic thromboembolic pulmonary hypertension (CTEPH). In recent decades, a refined surgical technique of PEA using a cardiopulmonary bypass (CPB) has achieved significant reduction in mortality. The hemodilution generated by crystalloid priming solutions in general heart surgery causes a number of disadvantages; no standard for PEA is described. We aim to assess the outcome of CTEPH patients after protocol change for CPB with adding $5 \%$ albumin to the priming solution during PEA.

Methods: Since changing the CPB protocol in 2018, 28 CTEPH patients were retrospectively compared to historical control by 1:1 propensity score matching gender, age, PVR (pulmonary vascular resistance) before PEA surgery. The resulting two cohort groups were analysed for intraoperative fluid balance, vasoactive-inotropic score (VIS), operative time and their influence on outcome. Regression models of hospitalization-, ICU (intensive care unit) and intubation time considered the total blood volume balance as mediator of the effect of the new protocol.

Results: Lower CPB-balance ( $\mathrm{p}<0.001)$, VIS score $(\mathrm{p}=0.04)$, shorter operative time $(\mathrm{p}=0.03)$ and hospitalization $(\mathrm{p}=0.008)$ were observed in the cohort operated with the new protocol. Postoperative 30 - and 90 day mortality and morbidity were similar between the two groups. Shorter operative time (mean change (mc) 1.4, 95\% CI [0.8. 2.0], $\mathrm{p}<0.0001)$ and new protocol ( $\mathrm{mc}-3.68$, $95 \%$ CI $[-5.22,-2.14], \mathrm{p}<0.0001)$ are independently associated with lower $\mathrm{CPB}$ balance, resulting both in a beneficial effect on ICU-, intubation- and hospitalization time and the VIS score (mean ratio $(\mathrm{mr}) 1.11,1.14,1.05,1.12, \mathrm{p}=0.0011,0.0013,0.008, \mathrm{p}<$ 0.0001 , respectively). The protocol change shows a clear positive trend (image) with significant impact on hospitalization time $(\mathrm{p}=0.02)$.

Conclusion: Priming protocol for CPB with addition of 5\% albumin had beneficial effect on $\mathrm{CPB}$ balance and improved postoperative outcome after PEA for CTEPH patients.

Disclosure: Isabelle Opitz has the following disclosures: AstraZeneca: Advisory Board; Medtronic: Institutional Grant; Roche: Speakers fee and Institutional Grant for Fellowship

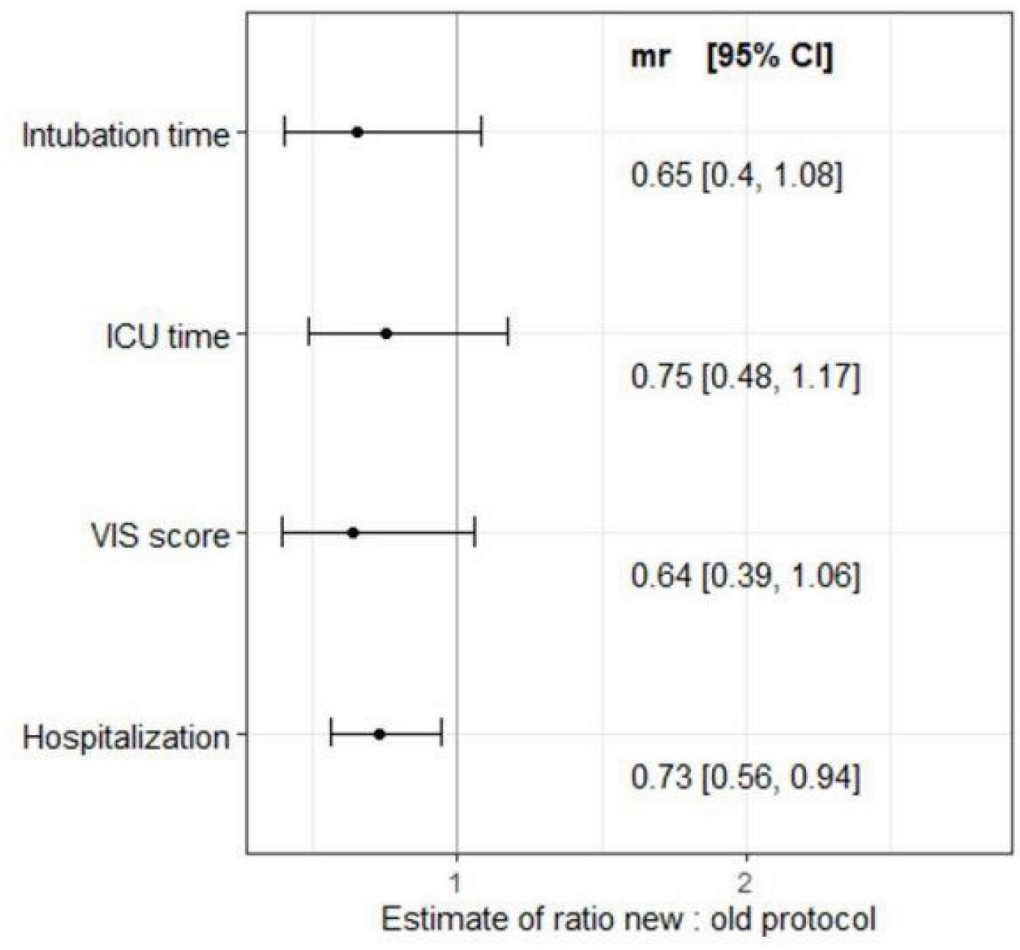

Fig. 1. Forest plot summarizing effects of protocol change.
Forest plot summarizing effects of protocol change on outcomes as derived from linear regression models with outcomes on logarithmic scale and considering the potential confounders New York Heart Association score before surgery and operative time. Mean ratios $(\mathrm{mr})$ and their $95 \%$ confidence intervals $(\mathrm{Cl})$ comparing the new with the old protocol are reported. 


\section{P64 \\ Molecular Characterization of the Local Tumor Microbiome in Lung Cancer Patients Treated with Checkpoint Inhibitors}

\author{
M. Boesch ${ }^{1}$, F. Baty ${ }^{1}$, M. Früh ${ }^{2}$, M. Joerger ${ }^{2}$, M.H. Brutsche ${ }^{1}$ \\ ${ }^{1}$ Lung Center, ${ }^{2}$ Department of Medical Oncology and \\ Hematology, Cantonal Hospital St. Gallen, St.Gallen, Switzerland
}

Introduction: Checkpoint inhibitors have revolutionized the treatment of cancer but only a fraction of patients respond favourably. The gut microbiome has been shown to influence checkpoint immunotherapy responsiveness in lung cancer and beyond, with a higher microbial diversity being generally associated with treatment benefit. In contrast, little is known about the functional role of the local tumor microbiome in cancer immunotherapy outcome.

Method: We employed bronchoscopic biopsies from checkpoint inhibitor-treated patients with non-small cell lung cancer, and analyzed these samples using next-generation 16S rRNA sequencing. Bacterial species were taxonomically allocated and their diversity (Shannon index) and abundance (read counts) was correlated to various clinical parameters including Kaplan-Meier survival.

Results: 16 S rRNA sequencing identified bacterial species from various phyla, with high proportions of firmicutes, bacteroidetes and proteobacteria detectable. Tumor bacterial signatures varied greatly and were characteristic of individual samples. A high diversity of the tumor bacterial habitat correlated significantly with improved overall survival (Figure 1A). In addition, high tumoral abundance of the class of gammaproteobacteria was associated with low PD-L1 expression and poor progression-free survival under checkpoint inhibitor therapy (Figure 1B).

Conclusion: We here show the feasibility of $16 \mathrm{~S}$ rRNA-based identification of bacteria in bronchoscopic biopsies from patients with non-small cell lung cancer. Specific features of the tumor microbiome have prognostic and predictive value for checkpointbased immunotherapy and might translate into novel therapeutic leads. Our study highlights the importance of the local tumor microbiome (as opposed to the distant gut microbiome) and sets the stage for further research to delineate the underlying mechanisms.

Disclosure: Nothing to disclose
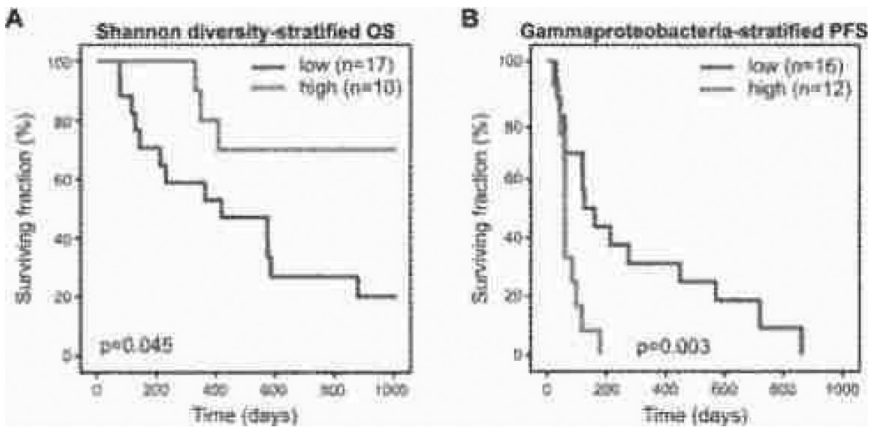

Fig. 1. Association of tumor microbial diversity and gammaproteobacteria with survival.

\section{P66 \\ Transbronchial Cryobiopsy Compared to Forceps \\ Biopsy for Diagnosis of Acute Cellular Rejection \\ in Lung Transplant Recipients: Analysis of $\mathbf{5 2}$ \\ Consecutive procedures}

\section{Steinack, A. Gaspert, J. Rüschoff, F. Gautschi, R. Hage, M. Schuurmans, D. Franzen}

Universitätsspital Zürich, Zürich, Switzerland

Aim/Purpose of the study: Acute cellular rejection (ACR) is a frequent complication in the first year after lung transplantation (LTx) and an important risk factor for chronic allograft dysfunction (CLAD) and mortality. Diagnosis of ACR is based on histologic findings using lung biopsies primarily by transbronchial forceps biopsy (FB). However, its diagnostic yield (DY) is limited due to the small biopsy size and crush artifacts. Transbronchial lung cryobiopsy (CB) is a more recent technique for diagnosis of interstitial lung diseases with improved DY compared to FB. However, there is insufficient evidence for $\mathrm{CB}$ in diagnosis and screening of ACR. This study aims to provide data on DY, safety, and impact on treatment decisions after $\mathrm{CB}$ in lung transplant recipients (LTRs).

Methods: Between December 2019 and January 2021, both FB and $\mathrm{CB}$ were obtained serially in LTRs during the same bronchoscopy session. According to our protocol, after 5 FB samples, 2 samples by $\mathrm{CB}$ were taken from different lung segments. The biopsies were scored according to the recent ISHLT criteria (A0 - A4) by a dedicated transplant pathologist, assessing FB and CB samples independently. We assessed the severity of bleeding with the Nashville Bleeding Scale (grade 1 - 4), and presence of pneumothorax was assessed by chest radiography.

Results: Totally, 52 consecutive procedures in 32 LTRs (29 males, median age 58) were performed either as routine surveillance bronchoscopy $(\mathrm{n}=41)$ or as clinically indicated bronchoscopy $(n=11)$. Retrospective analysis of $260 \mathrm{FB}$ and $51 \mathrm{CB}$ samples were performed. ACR (A1 - A3, minimal - moderate acute rejection) was detected in 17 cases (33\%) by CB resulting in a change of immunosuppressive strategy, compared to $4 \%$ by FB. CB showed bronchiolitis obliterans in 2 and cytomegalovirus pneumonitis in 1 case. The biopsy procedures were complicated by moderate (grade 2) bleeding in 19 (36\%) cases requiring repeat wedging or installation of diluted vasoactive substances and tranexamic acid. In one procedure bleeding grade 3 was observed demanding introduction of an endobronchial ballon blocker. Pneumothorax occurred in $4(8 \%)$ cases.

Conclusions: CB provided improved DY of ACR diagnosis with an acceptable safety profile, leading to reclassification and a change of treatment strategy in one third of cases.

Disclosure: Nothing to disclose 


\section{P67}

\section{Bilateral Elastofibroma Dorsi: A Case Report}

W. Saidani ${ }^{1}$, I. Bouassida ${ }^{1}$, B. Ben Radhia ${ }^{1}$, H. Zribi', A. Ben Mansour ${ }^{2}$, A. Dridi ${ }^{3}$, S. Zairi ${ }^{1}$, A. Marghli ${ }^{1}$

${ }^{1}$ Service de Chirurgie Thoracique, Hôpital Abderrahmen Mami, ${ }^{2}$ Service de Pneumologie, Hôpital Abderrahmen Mami, ${ }^{3}$ Service d'Anesthesie et de Réanimation, Hôpital Abderrahmen Mami, Ariana, Tunisia

Introduction: Elastofibroma dorsi (EFD) is a rare, benign, slow-growing chest wall tumor. It is characterized by a proliferation of elastic fibers within a stroma of collagenous adipose tissue. It is most commonly located at the posterior chest wall, in the infrascapular area, bordered by the subscapular, rhomboid, latissimus dorsi, and serratus anterior muscles. It is usually unilateral, and bilateral involvement occurs in only $10 \%$ of patients.

Observation: We report herein a case of a 62 -year-old man with bilateral EFD. He consulted for pain when moving his left shoulder. The patient was found to have bilateral involvement of the tumor following a physical examination with proper positioning. MRI demonstrated, in fact, two tumor masses under each scapula. The masses were oblong in shape, well circumscribed, nonencapsulated, showing a heterogeneous signal with a fibrillar structure with a fibrous component showing low signal intensity on T1- and T2-weighted sequences, and an adipose component showing high signal intensity on T1- and T2-weighted sequences, with slight enhancement after gadolinium injection. The diagnosis of EFD was made based on these imaging and clinical findings. Given the persistence of the progressively worsening functional discomfort, the patient underwent subsequent resections of the lesions. Histopathologically, it was consistent with a diagnosis of elastofibroma. Margins were tumor free. The patient was doing well after 6 months.

Conclusion: EFD is a benign pseudotumor with a characteristic MRI aspect comprising a stratified alternation of fibrous and fatty tissues. Although most often a unilateral lesion, elastofibromas may arise bilaterally. The possibility of a bilateral lesion must be kept in mind, especially when the lesion occurs in the infrascapular region. Surgical treatment can be proposed if the lesion is symptomatic or if doubt persists as to the lesion's benign nature: complete resection with healthy surgical margins, thus allowing precise histological diagnosis.

Disclosure: Nothing to disclose

\section{P68 \\ Malignant Pleural Mesothelioma: A Review of Three Cases}

\section{S. Abdala, S. Aitbatahar, L. Amro}

Department of Pneumology, Mohamed VI University Hospital Center, Marrakesh, Morocco

Introduction: Malignant pleural mesothelioma (MPM) is a rare tumor whose main risk factor is exposure to asbestos, which is found in $72-95 \%$ of cases.

Method: We report three observations of patients with MPM.

Results: All our patients were male, the average age was 70.5 years, exposure to asbestos was found in two patients, their profession was shoemaker, mechanic and welder, one patient was a smoker at 17 pack-years and a chronic cannabis user. Chest pain and dyspnea were found in all patients. The pleuropulmonary examination revealed a fluid effusion syndrome in all cases. Chest radiography showed an opaque hemithorax in two patients and pleurisy of moderate intensity in one case. The CT scan showed a liquid pleural collection and pleural thickening in all patients, while the extension essessement for extra thoracic neoplasia was normal. The diagnosis of mesothelioma was made by pleural biopsy in 2 patients, and by video-assisted surgical pleural biopsy in one patient, the treatment was based on chemotherapy.

Conclusion: The prognosis of malignant mesothelioma remains poor with a median survival of 7 to 17 months. Exposure to asbestos may be missing in a minority of cases.

Disclosure: Nothing to disclose

\section{EFP}

\section{P69 \\ Hémopneumothorax: Profil Étiologique et Évolutif}

A. Ajim, N. Ait mouddene, H. Arfaoui, H. Jabri, W. Elkhattabi, H. Afif

Service des Maladies Respiratoires, Hôpit al 20 Août 1953, CHU Ibn Rochd, Maroc

Introduction: L'hémopneumothorax est une pathologie de l'adulte jeune qui peut être secondaire à la rupture d'une bride pleurale au cours d'un pneumothorax, c'est une pathologie qui reste rare. Le but de ce travail est de déterminer le profil épidémiologique, clinique, paraclinique, étiologique et évolutif de Hémopneumothorax.

Méthode: Il s'agit d'une étude rétrospective portant sur 24 cas d'hémopneumothorax spontané colligé au service de pneumologie de janvier 2010 à janvier 2021. L'hémopneumothorax spon- 
tané et iatrogène sont inclus dans notre étude alors que l'hémopneumothorax traumatique est exclus de l'étude.

Résultats: La moyenne d'âge est de 33 ans, le tabagisme est retrouvé chez 16 cas, un antécédent de tuberculose pulmonaire traitée dans 6 cas et de pneumothorax drainé dans 2 cas. Le début des symptômes a été brutal dans la majorité des cas, le tableau clinique est dominé par la douleur thoracique associée à la dyspnée dans 18 cas. L'examen clinique a trouvé des signes de lutte respiratoire dans 3 cas, une pâleur cutanéo-muqeuse dans 14 cas et des adénopathies périphériques dans 11 cas; La radiographie thoracique a montré une image hydro-aérique dans tous les cas, un refoulement médiatisnal dans 4 cas, une opacité hilaire dans 2 cas et dans un seul une opacité paracardiaque ainsi qu'une image cavitaire. La ponction pleurale a ramené un liquide hémorragique incoagulable dans tous les cas, l'étude cytologique a montré ne prédominance lymphocytaire dans 12 cas et à polynucléaire neutrophile dans 6 cas; une anémie normocrhome normocytaire chez 13 cas et hypochrome microcytaire dans deux cas. La ponction biopsie pleurale a été en faveur d'une tuberculose dans 11 cas, métastatique dans 4 cas, une origine infectieuse probable dans 7 cas et indéterminée dans 2 cas. Le traitement est basé sur le drainage thoracique chez 20 cas et talcage par thoracoscopie dans 4 cas associé au traitement de l'étiologie causale ? L'évolution était bonne dans 22 cas, avec aggravation nécessitant un séjour en réanimation dans un seul cas et un cas décédé. Conclusion: L'hémopneumothorax est une pathologie peu fréquente dont le diagnostic étiologique reste difficile à déterminer et qui nécessite une prise en charge diagnostique et thérapeutique rapide.

Conflits d'intérêt: Rien à déclarer

\section{P70 \\ Qualité de vie Des Patients Atteints de Broncho-Pneumopathie Chronique Obstructive Dans Deux Structures Hospitalières à Yaoundé-Cameroun}

\author{
Nsounfon Abdou Wouoliyou, Massongo Massongo, \\ Poka-Mayap Virginie, Kuaban Alain, Ekongolo Marie Christine, \\ Pefura-Yone Eric Walter
}

Département de Médecine Interne et Spécialités, Faculté de Médecine et des Sciences Biomédicales, Université de Yaoundé I, Yaoundé, Cameroun

Introduction: L'évaluation et l'amélioration de la qualité de vie devraient faire partie de la prise en charge de la broncho-pneumopathie chronique obstructive (BPCO). La présente étude visait à évaluer la qualité de vie des patients atteints de BPCO, et identifier les facteurs associés à leur mauvaise qualité de vie.

Méthodologie: Nous avons mené une étude transversale descriptive et analytique à l'Hôpital Jamot de Yaoundé et la Clinique Polymère du 1er février au 30 juin 2020. Étaient inclus les patients adultes ( $\geq 21$ ans) atteints de BPCO et consentant. La qualité de vie a été évaluée à l'aide de deux questionnaires: le Saint George's Respiratory Questionnaire (SGRQ) et le 36-item Medical Outcomes Study Short-Form (MOS-SF 36). La mauvaise qualité de vie était définie par un score de SGRQ $\geq 30$. L'analyse des données a été faite à l'aide du logiciel IBM SPSS 23.0. La régression logistique multiple était utilisée pour identifier les déterminants de la mauvaise qualité de vie avec une significativité statistique fixé à 0,05 .

Résultats: Nous avons inclus 29 patients (58,6\% d'hommes) avec un âge moyen (écart type) de $64,7(14,2)$ ans. Les scores moyens (ET) de qualité de vie étaient de 44,8 $(23,9)$ pour le SGRQ et $49,06(21,9)$ pour le MOS-SF 36 . La prévalence (IC à 95\%) de la mauvaise qualité de vie était de 65,5 (48,3 - 82,8)\%. En analyse multivariée, seule la présence d'exacerbations au cours des 12 derniers mois $(\mathrm{RC}=12,3$; $\mathrm{IC}$ à $95 \%=1,1-136,7 ; \mathrm{p}=0,04)$ ressortait comme facteur associé indépendant à leur mauvaise qualité de vie.

Conclusion: La prévalence de la mauvaise qualité de vie était élevée chez les patients atteints de BPCO. Le facteur indépendamment associé à leur mauvaise qualité de vie était la présence d'exacerbations au cours des 12 derniers mois.

Conflits d'intérêt: Rien à déclarer

\section{P71 \\ Perception et Consommation de la Chicha Dans Une Population Estudiantine a Parakou, Benin}

\author{
Serge Ade ${ }^{1}$, Sylvanus Dingboe ${ }^{1}$, Gildas Agodokpessi, \\ Francis Tognon Tchegnonsi ${ }^{1}$ \\ ${ }^{1}$ Faculté de Médecine, Université de Parakou, Parakou, Bénin, \\ ${ }^{2}$ Faculté des Sciences de la Santé, Université d'Abomey-Calavi, \\ Cotonou, Bénin
}

Introduction: Ces dernières années, l'on assiste à un engouement grandissant des jeunes en Afrique subsaharienne pour la chicha, un mode de consommation du tabac plus aromatisé, plus humidifié, moins irritant, mais plus toxique que la fumée de la cigarette. L'objectif de ce travail était d'étudier la perception et la consommation de la chicha chez des étudiants de l'Université de Parakou.

Méthode: Il s'agissait d'une étude transversale prospective menée en 2019. La taille de l'échantillon, déterminée à partir de la formule de Schwartz était 570 . Un sondage en grappes était réalisé. Les participants étaient invités à remplir de façon confidentielle un questionnaire auto-administré.

Résultats: Au total, 72,28\% des enquêtés percevaient la chicha comme moins nocive que la cigarette, surtout du fait de l'odeur aromatisé $(37,29 \%)$ et du passage de la fumée par le liquide (26,88\%). Ils étaient 25,09\% à en avoir consommé au moins une fois dans la vie; et $9,13 \%$ à en fumer le mois dernier. Les principales motivations étaient le plaisir $(58,74 \%)$, l'imitation des amis $(49,65 \%)$ et l'odeur agréable $(46,85 \%)$. Les facteurs de risque de consommation de chicha retrouvés étaient l'exposition passive à sa fumée dans les bars/restaurants $(R R=2,85 ; \mathrm{p}=0,001)$ et dans les boîtes de nuit $(\mathrm{RR}=3,56 ; \mathrm{p}=<0,001)$ alors que les facteurs protecteurs étaient la nocivité connue de la chicha $(\mathrm{RR}=0,41$; $\mathrm{p}=0,015)$ et une gêne ressentie lors de l'inhalation passive de la fumée $(R R=0,18 ; p=<0,001)$. 
Conclusion: La majorité des étudiants ignore la nocivité de la chicha. La prévalence de la consommation de la chicha parmi les étudiants est élevée. Son initiation est fortement influencée par l'entourage. Il urge de mener des actions de sensibilisation vis-àvis de cette forme de consommation du tabac.

Conflits d'intérêt: Rien à déclarer

\section{P72 \\ Caractéristiques Cliniques des Patients Atteints de Sarcoïdose Médiastinopulmonaire: Étude de Série de 80 Cas}

\section{F. Adila, F. Ziane, A. Zitouni}

Service de Pneumologie, Hôpital Central de l'Armée. Alger, Algérie

Introduction: La sarcoïdose est une maladie granulomateuse systémique qui affecte le poumon et les ganglions lymphatiques dans plus de $85 \%$ des cas, mais elle peut toucher n'importe quel organe. Les atteintes ophtalmologiques, dermatologiques et ganglionnaires périphériques sont les localisations extra pulmonaires les plus fréquentes dans 10 à $30 \%$ des cas (1). La présentation clinique de cette affection est polymorphe et le délai entre l'apparition des premiers symptômes et l'établissement du diagnostic est relativement long (2).

Méthodes: Notre étude est prospective, longitudinale et descriptive, concernant 80 cas de sarcoïdose médiastinopulmonaire prouvée à l'histologie, associée ou non à d'autres localisations extra pulmonaires. Tous nos patients étaient candidats à un traitement systémique par corticothérapie orale.

Résultats: Le début des troubles variait entre 0 et 72 mois avec une moyenne de $16.38 \pm 3.32$ mois. Au moment du diagnostic, $100 \%$ des patients avaient présenté des symptômes respiratoires, $76.25 \%$ des signes fonctionnels extra respiratoires et $71.25 \%$ des signes généraux. La dyspnée était présente chez $90.85 \%$ des cas, la toux sèche dans $85 \%$ et la douleur thoracique dans $48.75 \%$ des cas.

Les principaux signes extra respiratoires étaient les arthralgies, retrouvées dans $45.90 \%$ des cas; les adénopathies périphériques dans $39.34 \%$ et les nodosités sarcoïdosiques cutanées dans $35.48 \%$ des cas. L'atteinte ganglionnaire périphérique la plus fréquente était la chaine cervicale bilatérale avec $54.20 \%$ des cas; les adénopathies sarcoïdosiques dans notre étude étaient indolores, fermes, mobiles et sans signes inflammatoires en regard dans $91.70 \%$ des cas. L'érythème noueux était présent dans $54.30 \%$ des cas. Le maitre symptôme général était l'asthénie, retrouvé chez $86 \%$ des cas; l'amaigrissement était retrouvé chez $58 \%$ des cas, variant entre 03 et $15 \mathrm{Kg}$ avec une moyenne de $6.73 \pm 0.91 \mathrm{Kg}$.

Conclusion: La sarcoïdose peut prendre n'importe quelle présentation clinique thoracique et extra thoracique. Aucun signe n'est spécifique à lui seul, ce qui rend le diagnostic parfois difficile et souvent tardif. Cette pathologie mérite une attention particulière de la part des cliniciens afin de raccourcir au maximum le délai du diagnostic pour une meilleure prise en charge thérapeutique.

Conflits d'intérêt: Rien à déclarer

\section{P73 \\ L'exposition à la Fumée du bois: Facteur de Risque du Carcinome Bronchogénique}

H. Anniche, H. Benjelloun, N. Zaghba, N. Yassine

Service des maladies respiratoires $\mathrm{CHU}$ Ibn Rochd, Casablanca, Maroc

Introduction: Le rôle de l'environnement domestique chez la femme en milieu rural, notamment l'exposition à la fumée du bois a été reconnu par l'OMS comme facteur de risque du carcinome bronchogénique chez la femme. L'objectif de notre étude est d'évaluer le profil des patientes porteuses de carcinome bronchogénique et exposées à la fumée du bois.

Méthode: Nous avons mené une étude rétrospective portant sur 55 patientes vivant en milieu rural, exposées à la fumée du bois, admises pour un carcinome bronchique primitif entre janvier 2015 et juin 2020 au service des maladies respiratoires du CHU Ibn Rochd de Casablanca. Les patientes tabagiques ou exposées au tabagisme passif ont été exclues de cette étude.

Résultats: La moyenne d'âge était de 65 ans (41-88 ans), toutes les femmes habitaient en milieu rural et étaient exposées à la fumée du bois: cuisson au feu du bois dans tous les cas. L'exposition était quotidienne et régulière avec une durée moyenne de 34 ans. Le délai moyen de consultation était de 70 jours. Alors que le délai moyen entre l'exposition et l'apparition des symptômes était de 14 ans. La symptomatologie était faite d'une dyspnée d'effort (82\%), d'un syndrome bronchique (75\%), d'une douleur thoracique (70\%) et d'hémoptysie (30\%). L'examen clinique était normal dans $53 \%$ des cas. La tomodensitométrie a objectivé un processus médiastino-pulmonaire dans $90 \%$ des cas, associé à une pleurésie dans $36 \%$ des cas. La preuve histologique était obtenue par les biopsies bronchiques dans 29 cas, par la ponction biopsie transpariétale dans dix cas et par la ponction biopsie pleurale dans neuf cas. L'adénocarcinome représente le type histologique le plus fréquent dans $58 \%$ des cas. Au total, la plupart des cas étaient classées stade IV. Soixante-dix-neuf pour cent des femmes ont bénéficié d'un traitement palliatif. Une association radio-chimiothérapie était indiquée chez quatre patientes, une immunothérapie chez deux patientes, et six femme sont décédées.

Conclusion: Nous insistons à travers cette étude sur la relation de cause à effet entre l'exposition à la fumée du bois et la survenue du carcinome bronchogénique chez les personnes exposées et notamment les femmes vivant en milieu rural.

Conflits d'intérêt: Rien à déclarer 


\section{P74}

\section{L'œdème Pulmonaire à Vacuo: À Propos de 03 Cas}

Y. Bougrini, S. Zouiten, Z. Biaz, B. Amara, MC. Benjelloun, M. Serraj

Service de Pneumologie, CHU Hassan II FES Faculté de

Médecine et de Pharmacie de FES Université Sidi Mohamed Ben

Abdellah Fes, Maroc

Introduction: L'œdème pulmonaire à vacuo est un accident rare mais potentiellement sévère avec une mortalité de 15 à $20 \%$. Nous rapportons 3 observations d'OAP à vacuo après drainage d'un pneumothorax.

Cas cliniques: Un jeune patient de 19ans, sans antécédents pathologiques, admis au service de pneumologie pour prise en charge d'un pneumothorax primaire idiopathique. Le 2ème malade est un patient de 48 ans, tabagique chronique, admis pour pneumothorax spontané. Le 3ème malade est un homme de 56ans, ex-tabagique chronique, suivi pour BPCO stade B, admis pour prise en charge d'un pneumothorax secondaire post-traumatique (chute de sa hauteur). La durée d'évolution de symptômes était de $48 \mathrm{~h}$ à 15 jours, l'examen d'admission trouvait le lere malade stable, alors que les deux autres étaient dyspnéiques au repos et dépendants d'oxygénothérapie. La radiographie thoracique des trois patients objectivait la présence d'un pneumothorax unilatéral total compressif. Le drainage pleural a été réalisé et a permis d'évacuer les pneumothorax en quelques minutes. Quelques heures plus tard, un œdème pulmonaire radiologique avec retentissement clinique a compliqué le drainage. L'évolution a été favorable avec un traitement symptomatique en quelques jours.

Discussion: L'œdème pulmonaire unilatéral à vacuo est une affection rare. Il complique le plus souvent le drainage de pneumothorax, mais aussi après évacuation d'une pleurésie ou la réexpansion d'une atélectasie. Certains facteurs semblent favoriser la survenue d'un œè̀me à vacuo, tels que la durée et l'importance du collapsus pulmonaire ainsi que la rapidité de réexpansion du poumon après drainage justifiant ainsi certaines mesures de précaution. Le diagnostic, orienté par l'anamnèse et le contexte, et posé devant un tableau clinique et radiologique d'OAP unilatéral du côté de la réexpansion. Le traitement curatif repose essentiellement sur l'oxygénothérapie et la diminution des pressions d'aspiration pleurale.

Conclusion: le meilleur traitement reste la prévention, c'est-àdire, l'évacuation fractionnée sans dépression et avec une réexpansion lente.

Conflits d'intérêt: Rien à déclarer

\section{P75 \\ Abcès Pulmonaire Compliqué d'une Pleurésie Purulente}

A. Rattal, H. Benjelloun, N. Zaghba, N. Yassine

Service des maladies respiratoires $\mathrm{CHU}$ Ibn Rochd, Casablanca, Maroc

Introduction: L'abcès pulmonaire est une suppuration collectée dans une cavité néoformée, creusée dans le parenchyme par une infection aiguë non tuberculeuse. Parmi ses complications aiguës, la pleurésie purulente. L'objectif de notre étude est d'évaluer le profil des patients ayant présenté un abcès pulmonaire qui s'est compliqué d'une pleurésie purulente.

Méthode: Une étude rétrospective a été menée entre janvier 1995 et juin 2020 au service des maladies respiratoires du CHU Ibn Rochd de Casablanca portant sur 129 cas d'abcès pulmonaire dont 22 compliqués de pleurésie purulente, soit $17 \%$.

Résultats: La moyenne d'âge était de 40 ans avec une nette prédominance masculine. Le tabagisme était retrouvé dans 17 cas, le diabète dans 6 cas, l'insuffisance rénale dans deux cas, l'otite chronique, le post-partum, et la syphilis tertiaire dans un cas chacun. Le tableau clinique était brutal dans $75 \%$ des cas, dominé par la douleur thoracique dans tous les cas et la fièvre dans $91 \%$ des cas. Le syndrome d'épanchement liquidien était retrouvé chez tous les patients. Le téléthorax avait montré une opacité de type pleural homolatérale à l'abcès dans tous les cas, prédominante à droite dans $62 \%$ des cas. Le liquide pleural était purulent chez tous les patients. L'étude bactériologique De celui-ci avait isolé un Streptococcus bêta-hémolytique dans 8 cas et un bacille gram négatif dans 7 cas. La bronchoscopie avait montré un corps étranger endobronchique dans un cas. Le traitement était à base d'une bi-antibiothérapie dans tous les cas, un drainage thoracique d'emblée dans 11 cas, des ponctions pleurales évacuatrices biquotidiennes dans 7 cas avec une kinésithérapie respiratoire dans tous les cas. L'évolution clinique et radiologique était bonne dans $72 \%$ des cas. Six cas sétaient compliqués de pachypleurite.

Conclusion: La pleurésie purulente est une complication assez fréquente de l'abcès pulmonaire, qui est source de complications parfois invalidantes d'où l'intérêt du traitement précoce et adapté de ce dernier.

Conflits d'intérêt: Rien à déclarer 


\section{P76}

\section{La Tuberculose Endobronchique}

\section{A. Boussehra, N. Zaghba, H. Benjelloun, N. Yassine}

Service des maladies respiratoires du CHU Ibn Roch de Casablanca

Introduction: La tuberculose endo-bronchique est une forme particulière de la tuberculose qui reste peu fréquente, se définit comme une infection tuberculeuse de l'arbre trachéo-bronchique associée ou non à une atteinte parenchymateuse.

Méthode: Notre étude rétrospective porte sur 20 cas de tuberculose endo-bronchique colligés de janvier 2016 à décembre 2019 au service des maladies respiratoires du CHU Ibn Roch de Casablanca Le but du travail est de décrire les aspects cliniques et radiologiques de cette forme de tuberculose

RésuItats: Il s'agissait de 13 femmes et 7 hommes, âgés en moyenne 48 ans, sans aucun antécédent de tuberculose ni de contage tuberculeux dans l'entourage. Le tableau clinique était dominé par la toux et la dyspnée d'effort. L'hémoptysie était retrouvée dans huit cas. La radiographie thoracique montrait des lésions pulmonaires associées dans 15 cas. La bronchoscopie avait montré des granulations blanchâtres endo-bronchiques dans 12 cas et un bourgeon d'allure tumorale dans 3 cas. Les biopsies bronchiques avaient conclu à une tuberculose caséo-folliculaire dans tous les cas. La ponction biopsie pleurale avait confirmé une tuberculose pleurale associée dans 1 cas. La recherche de bacille de Koch (BK) dans les expectorations était positive dans 10 cas et à la culture dans 7 cas. Le traitement anti-bacillaire était prescrit chez tous nos patients associés à une corticothérapie orale dans cinq cas. L'évolution était satisfaisante dans tous les cas.

Conclusion: La tuberculose endo-bronchique est caractérisée par son polymorphisme clinique et radiologique pouvant simuler d'autres diagnostics et retarder ainsi sa prise en charge. Il faut y penser devant une toux persistante avec altération de l'état général dans un pays d'endémie. Nous soulignons l'intérêt de la bronchoscopie ainsi que les examens bactériologiques et histologiques qui ont eu un rôle non négligeable dans la confirmation de la tuberculose endo-bronchique dans notre série

Conflits d'intérêt: Rien à déclarer

\section{P77}

\section{L'atteinte Thoracique au Cours du Myélome Multiple}

\section{H. Bamha, M. Zadi, H. Arfaoui, H. Jabri, W. Elkhattabi, H. Afif}

Service des Maladies Respiratoires, Hôpital 20 août 1953, $\mathrm{CHU}$ Ibn Rochd

Introduction: Le myélome multiple est une prolifération plasmocytaire maligne caractérisée par l'infiltration de la moelle osseuse par des plasmocytes synthétisant une immunoglobuline monoclonale complète ou incomplète.

Méthode: Afin de décrire les différents aspects cliniques, radiologiques et biologiques du myélome avec localisation thora- cique, nous avons réalisé une étude rétrospective sur une durée de 8 ans (2012-2020) incluant 13 patients atteints de myélome multiple avec une localisation thoracique, hospitalisés au service de pneumologie de l'hôpital 20 aout de Casablanca.

Résultats: Il s'agit de neuf hommes et quatre femmes. La moyenne d'âge était 60 ans. Des douleurs osseuses diffuses sont notées dans $82 \%$ des cas dont les douleurs thoraciques (50\%), des hémoptysies (53\%), une masse pariétale (42\%), une fièvre (30\%) et une altération de l'état général (100\%). L'imagerie thoracique avait montré une opacité périphérique ( 8 cas) et une lyse costale $(7 \mathrm{cas})$. La triade: atteinte rachidienne, vitesse de sédimentation accélérée et protéinurie de Bence-Jones positive était trouvée dans $82 \%$ des cas. Le diagnostic histologique du myélome multiple était posé par le myélogramme (6 cas), par la biopsie ostéomédullaire (4 cas), biopsie de la masse pariétale ( 3 cas). Neuf patients avaient bénéficié d'une chimiothérapie. La radiothérapie était prescrite chez trois patients présentant des douleurs osseuses persistantes et un patient a décédé avant de démarrer le traitement. L'évolution est marquée par l'aggravation.

Conclusion: Les localisations thoraciques du myélome sont rares et leur prise en charge doit être pluridisciplinaire. Le pronostic du myélome multiple est tout de même mauvais.

Conflits d'intérêt: Rien à déclarer

\section{P78 \\ Les Atteintes Cardio-Vasculaires au Cours de la Broncho-Pneumopathie Chronique Obstructive}

\section{W. Elkhattabi, H. Bamha, H. Arfaoui, H. Jabri, H. Afif}

Service des Maladies Respiratoires, Hôpital 20 Août 1953, CHU Ibn Rochd

Introduction: Les atteintes cardio-vasculaires au cours de la broncho-pneumopathie chronique obstructive sont fréquentes, dominées par le cœur pulmonaire chronique, l'hypertension arterielle pulmonaire et l'insuffisance coronarienne. Le but de notre travail est d'évaluer la prévalence de ces complications dans la $\mathrm{BPCO}$ et de décrire le profil des patients présentant ces affections.

Méthode: Il s'agit d'une étude rétrospective incluant 109 cas étalée sur 5 ans (juin 2015 au juin 2020).

Résultats: Parmi 286 cas hospitalisés pour exacerbation de BPCO,109 malades ont des complications cardio-vasculaires $(38,1 \%)$ retenues sur la clinique, l'ECG et l'échocardiographie. La moyenne d'âge est de 66,2 ans. Tous les malades sont de sexe masculin, le tabagisme est noté chez tous les patients (une moyenne de 28 paquets-année), $51 \%$ sont non sevrés, $23 \%$ sont consommateurs de cannabis, le nombre moyen d'exacerbations est de trois par an. Le début de la maladie est en moyenne de 9 ans, $77 \%$ sont de nos patients sont non observant du traitement. La BPCO est classée stade C dans $71 \%$ des cas et stade D dans 25\%. L'ECG trouve une ACFA dans 21 cas soit 19,2\%, une HVD dans 45 cas soit $41,2 \%$, un bloc de branche droit chez 16 cas soit $14,6 \%$. L'échocœur objective une HTP chez 36 cas soit $33 \%$, une hypertrophie du ventricule droit chez 28 cas soit $25,7 \%$, et une insuffisance cardi- 
aque globale chez 20 cas soit 18,3\%. Le traitement est basé sur les diurétiques, les antiarythmiques, les digitaliques et les anticoagulants, associé au traitement de l'exacerbation et au traitement de fond de la BPCO. L'évolution est favorable faite d'une régression du nombre des exacerbations et une amélioration de la fonction respiratoire pour $60 \%$ des patients.

Conclusion: Les comorbidités cardiovasculaires représentent un nouvel enjeu médical et thérapeutique dans la BPCO, elles constituent un élément incontournable par leur fréquence élevée, leur impact sur la qualité de vie et leur valeur pronostique vitale des patients. D'où la nécessité de les rechercher systématiquement et de les traiter.

Conflits d'intérêt: Rien à déclarer

\section{P79 \\ Le Diagnostic Clinique et Radiologique des Abcès Pulmonaires}

\author{
W. Elkhattabi, H. Bamha, L. Elhamdaoui, A. Fahmi, H. Arfaoui, \\ H. Jabri, H. Afif \\ Service des Maladies Respiratoires, Hôpital 20 Août 1953, \\ CHU Ibn Rochd
}

Introduction: L'abcès du poumon est défini comme une zone circonscrite de nécrose parenchymateuse d'origine infectieuse générant une ou plusieurs cavités suppurées. Sa fistulisation bronchique est responsable de l'apparition d'un niveau hydro-aérique visualisé en imagerie.

Méthode: Notre étude est rétrospective portant sur 104 patients hospitalisés au service entre janvier 2004 et janvier 2021 au service de pneumologie de l'hôpital 20 Aout de Casablanca.

Résultats: Il s'agit de 82 hommes $(78,9 \%)$ et 22 femmes $(21,1 \%)$ avec une moyenne d'âge de 47 ans. Le tabagisme actif est noté dans $44 \%$ et l'alcoolisme chronique dans $7 \%$ des cas. Le diabète est retrouvé chez 17 patients (16,3\%). Le début était brutal dans $70 \%$ des cas. L'association douleur thoracique et syndrome bronchique étaient le mode de révélation le plus fréquent dans $94 \%$ des cas. $\mathrm{La}$ fièvre était présente dans $58 \%$ des cas. La radiographie thoracique a montré une image hydro-aérique dans la majorité des cas prédominante au côté droit, elle est isolée dans $85 \%$ et associée à des opacités alvéolaires dans $20 \%$ des cas. Une pleurésie est associée dans 23\%. La TDM thoracique faite chez 52 patients a objectivé en plus des images d'abcès, des processus tissulaires périphériques chez 8 patients dont la ponction-biopsie transpariétale scanno-guidée a confirmé un carcinome bronchogénique. La bronchoscopie est réalisée chez 48 patients a objectivé une inflammation du 2e degré. L'étude cytobactériologique des expectorations et du liquide d'aspiration bronchique ont permis d'isoler un germe chez 39 patients (37,5\%). Les germes isolés étaient représentés essentiellement par le pneumocoque et les bacilles gram négatif. La prise en charge thérapeutique est basée sur le traitement des facteurs favorisants, une bi-antibiothérapie à large spectre adaptée associée à une kinésithérapie de drainage bronchique et des ponctions pleurales évacuatrices. L'évolution était bonne dans 95 cas $(91,3 \%)$ avec nettoyage radiologique. Parmi les facteurs favorisants retrouvés: le diabète dans $16,3 \%$ des cas, l'alcoolisme chronique dans $7 \%$ des cas. Nous déplorons le décès de 8 cas dans un tableau de septicémie.

Conclusion: L'abcès du poumon est devenu moins fréquent depuis le traitement antibiotique systématique à l'origine de certaines formes frustes, toutefois sa survenue sur un terrain fragilisé peut avoir des conséquences graves.

Conflits d'intérêt: Rien à déclarer

\section{P80 \\ Les Moyens de Confirmation de Carcinome Bronchogénique}

\section{A. Boumehdi, A. Ajim, H. Arfaoui, H. Jabri, W. Elkhattabi, H. Afif}

Service des Maladies Respiratoires, Hôpital 20 Août 1953, CHU Ibn Rochd

Introduction: Le cancer bronchique primitif (CBP) est le cancer masculin le plus fréquent dans lemonde. Son incidence ne cesse d'augmenter chaque année suivant en cela la consommation tabagique. Le diagnostic de certitude de ce cancer repose sur les résultats de l'examen anatomopathologique.

Méthode: Nous rapportons une étude rétrospective portant sur 620 cas de carcinome bronchique dont nous avons analysé le profil épidémiologique radio-clinique et les moyens de diagnostic.

Résultats: La moyenne d'âge de nos patients est de 60 ans, avec une nette prédominance masculine $95 \%$. Quatre-vingt-dix pourcents des patients sont tabagiques avec une moyenne de 40 paquets années. La douleur thoracique est retrouvéedans $73 \%$, l'hémoptysie dans $35 \%$. Le scanner thoracique a confirmé la nature tissulaire des lésions radiologiques dont le siège est proximal dans 59\% et distale dans $41 \%$. Les différents types histologiques retrouvés sont l'adénocarcinome dans $40 \%$, le carcinome épidermoide (31\%), le carcinome indifférencié (17\%), le carcinome neuroendocrine (10\%) et lymphangite carcinomateuse (2\%) dont le moyen de confirmation était par biopsie bronchique dans 52\%, ponction transpariétale-échoguidée dans $22 \%$, ponction biopsie transpariétales - scanoguidée dans $13 \%$, biopsie ganglionnaire dans $4 \%$, ponction biopsie pleurale dans $6 \%$ et biopsie d'un nodule cutané dans un cas.

Conclusion: Le choix des techniques de prélèvements du site tumoral dépend de la localisation de la lésion tumorale déterminée par un examen clinique complet et par l'imagerie, l'état général du patient et de ses comorbidités.

Conflits d'intérêt: Rien à déclarer 


\section{P81 \\ Foyer de Condensation Pulmonaire Chronique: Profil étiologique (à Propos de 70 Cas)}

\section{W. Elkhattabi, R. Cherkaoui, H. Arfaoui, H. Jabri, H. Afif}

Service des Maladies Respiratoires, Hôpital 20 Août 1953, CHU Ibn Rochd

Introduction: De nombreuses étiologies peuvent être responsables d'un tableau radiologique de condensation pulmonaire chronique, situation fréquente en pratique clinique.

Méthode: Le but de notre étude est de déterminer le profil étiologique de foyer de condensation pulmonaire chronique à travers une étude rétrospective portant sur 60 patients hospitalisés au service entre janvier 2014 et juillet 2020 .

Résultats: La moyenne d'âge est de 58 ans, avec une nette prédominance masculine $(67,8 \%)$. Les antécédents sont dominés par le tabagisme actif dans $60 \%$ des cas suivi par le diabète dans $15 \%$ des cas. Le tableau clinique est d'installation progressive dans la totalité des cas, dominés par la dyspnée dans 90\% des cas, l'altération de l'état général dans $72,5 \%$ des cas et le syndrome bronchique dans $45,6 \%$ des cas. L'examen clinique a trouvé un syndrome de condensation alvéolaire dans 35,7\% des cas. La tomodensitométrie thoracique a objectivé un foyer de condensation alvéolaire bilatéral dans $71,4 \%$ des cas. La bronchoscopie souple a montré un bourgeon tumoral dans $10 \%$ des cas et une sténose infilrative dans $8,3 \%$ des cas. Le diagnostic étiologique est apporté par la ponction biopsie transpariétale dans $53,3 \%$ des cas, la bronchoscopie souple dans $26,7 \%$ des cas, la recherche des germes dans les aspirations bronchiques et la ponction biopsie pleurale dans $10 \%$ des cas pour chacun. Les étiologies sont dominées par l'adénocarcinome pulmonaire dans $46,6 \%$ des cas, suivi par la tuberculose pulmonaire dans $26,6 \%$ des cas, les pneumonies bactériennes dans 18,3\% des cas et d'autres étiologies rares dans $8,5 \%$ des cas. L'évolution a été bonne dans $66 \%$ des cas.

Conclusion: A travers cette étude on souligne la diversité étiologique du foyer de condensation pulmonaire, qui reste dominer par le carcinome bronchogénique et la tuberculose pulmonaire dans notre contexte.

Conflits d'intérêt: Rien à déclarer

\section{P82}

\section{Pneumothorax Secondaire: Particularités Par Rapport au Pneumothorax Primitif}

\section{E. Ben Jemia, A. Allouch, H. Zaibi, K. Hemissi, K. Euchi, w. Rejeb, J. Ben Amar, H. Aouina}

Service de pneumologie, Hôpital Charles Nicolle, Tunisie Faculté de médecine de Tunis, Université Tunis El Manar

Introduction: Le pneumothorax spontané peut être primitif ou secondaire à une pathologie pulmonaire sous-jacente. Notre objectif est d'étudier les particularités cliniques, radiologiques, thérapeutiques et évolutives des pneumothorax secondaires par rapport au pneumothorax primitif.

Méthode: Nous avons mené une étude rétrospective et descriptive ayant inclus 140 patients hospitalisés au service de pneumologie de l'hôpital Charles Nicolle de Tunis pour pneumothorax spontané sur une période de 12 ans (2008-2020). Les patients étaient répartis en 2 groupes: G1 ( $\mathrm{n}=94)$ patients ayant un pneumothorax primitif; G2 $(\mathrm{n}=46)$ patients ayant un pneumothorax secondaire.

Résultats: Dans le premier groupe G1, les patients étaient plus jeunes (32 vs 51 ans; p\&lt;0,0045). Les hommes étaient majoritaires dans les 2 groupes, et la plupart des patients étaient fumeurs avec une consommation moyenne de 14 PA dans G1 et 42 PA dans G2 $(\mathrm{p}=0,01)$. Sur le plan fonctionnel, le tableau clinique était dominé par la douleur thoracique et la dyspnée dans les deux groupes. Le délai entre l'apparition des symptômes et la consultation était de 2,4 jours dans G1, et 4,5 jours dans G2 (p\&gt;0.05). Il n'y avait pas de différence entre les deux groupes quant au caractère total ou partiel du pneumothorax. La radiographie thoracique avait montré des lésions parenchymateuses associées plus fréquentes chez les patients de $\mathrm{G} 2: 12$ patients ( $13 \%$ ) dans $\mathrm{G} 1$ contre 35 patients $(76 \%)$ dans G2 (p\&lt;0,001). Le recours à un scanner thoracique était nécessaire chez $40 \%$ des patients dans les 2 groupes. La durée moyenne de drainage thoracique était significativement plus prolongée pour le G2 (8,2 vs 11,7 jours, $\mathrm{p}=0,03)$. Sept patients du G1 avaient eu recours à la chirurgie, contre 9 patients du G2. La récidive était plus fréquente en cas de pneumothorax secondaire $(11,7 \%$ vs $17,4 \% ; p=0,017)$. Dans $G 1$, la récidive était homolatérale chez 10 patients, et controlatérale chez un seul patient. Dans G2, elle était homolatérale chez 6 patients, et controlatérale chez 3 patients, sans différence significative entre les 2 groupes.

Conclusion: Le pneumothorax secondaire semble toucher des patients plus âgés, ayant une consommation tabagique plus importante. Sa prise en charge est urgente vu l'état pathologique du poumon sous-jacent.

Conflits d'intérêt: Rien à déclarer

\section{P83 \\ Caractéristiques Cliniques et Évolutives de la Tuberculose Pulmonaire Chez Les Patients Diabétiques}

E. Ben Jemia, M. Mosbah, K. Hemissi, H. Zaibi, A. Naaroura, K. Euchi, J. Ben Amar, H. Aouina

Service de pneumologie, Hôpital Charles Nicolle, Tunisie Faculté de médecine de Tunis, Université Tunis El Manar

Introduction: De nombreuses études ont démontré que les sujets diabétiques ont un risque plus élevé de développer une tuberculose maladie (TBC). L'objectif de cette étude était de déterminer les particularités cliniques et évolutives de la tuberculose pulmonaire chez les sujets diabétiques (D).

Méthode: Etude rétrospective et comparative portant sur des patients hospitalisés pour TBC au service de pneumologie de 
l'hôpital Charles Nicolle de Tunis, entre janvier 2018 et aout 2020. (D+): sujets diabétiques; (D-): sujets non diabétiques.

Résultats: Nous avons inclus 71 patients, dont $12,7 \%(n=9)$ étaient diabétiques. L'âge moyen était de $45 \pm 14$ ans chez les (D+) vs $39 \pm 15,8$ ans chez les (D-) ( $p=0.007)$. L'IMC moyen était comparable pour les deux groupes (D+: $19 \pm 1,4$ vs D-: $18,8 \pm 2,4 \mathrm{~kg} / \mathrm{m} 2$; $\mathrm{p}=0,09)$. La présentation clinique et radiologique de la TBC ne différait pas entre les deux groupes. Le principal moyen diagnostique était la présence de bacille alcoolo-acido-résistant (BAAR) dans les expectorations. Les patients $(\mathrm{D}+)$ présentaient plus de localisations extra-pulmonaires de tuberculose $(\mathrm{p}=0,02)$. L'observance thérapeutique était satisfaisante dans $78 \%$ des cas sans différence entre les 2 groupes. Le délai de prise du poids était plus long chez les $(D+)(31 \pm 23$ jours vs $25 \pm 14$ jours, $p=0.04)$. Le délai de négativation des BAAR dans les expectorations et le délai d'amélioration radiologique ne différaient pas entre les 2 groupes ( $p>0.05)$. Les séquelles pulmonaires étaient plus nombreuses chez les patients $(\mathrm{D}+)(\mathrm{p}=0,001)$, tandis que la récidive de la maladie ne différait pas entre les 2 groupes $(\mathrm{p}=0,09)$.

Conclusion: La tuberculose pulmonaire des patients diabétiques se caractérise par une maladie plus disséminée, une évolution plus trainante avec un risque de séquelle plus important.

Conflits d'intérêt: Rien à déclarer

\section{P84 \\ Particularité du Carcinome Bronchogénique Chez le Non Fumeur}

\section{H. Arfaoui, R. Cherkaoui, H. Jabri, W. Elkhattabi, H. Afif}

Service des Maladies Respiratoires, Hôpital 20 Août 1953, CHU Ibn Rochd

Introduction: Le cancer bronchique est la première cause de décès chez l'homme dans le monde. Son incidence chez les patients sans habitudes toxiques est augmentée ces dernières années.

Méthode: Afin de déterminer le profil clinique, histologique, thérapeutique et évolutif chez le sujet sans habitudes toxiques, nous avons mené une étude rétrospective à propos de 110 cas allant de juillet 2011 à décembre 2019.

Résultats: Les hommes ont représenté $52 \%$ avec une moyenne d'âge de 61 ans, le tabagisme passif est retrouvé chez 21 patients (19\%), une exposition professionnelle aux substances cancérigènes est notée chez 12 patients (11\%) dont l'amiante. La dyspnée, la douleur thoracique et la toux sèche ont été les maitres symptômes avec respectivement $90 \%, 81 \%$ et $77 \%$. La TDM thoracique a objectivé un processus périphérique dans $54 \%$ des cas, une pleurésie dans $30 \%$ des cas et un trouble de ventilation dans $10 \%$ des cas. Le diagnostic histologique a été obtenu par les biopsies transpariétale dans $25 \%$ des cas, et par une ponction biopsie pleurale dans $22 \%$ des cas. L'adénocarcinome a représenté le type histologique prédominant (48\%), le carcinome épidérmoide est retrouvé dans $20 \%$ des cas. Quarante-six pourcent des patients sont classés stades IIIB et IV. Seulement $10 \%$ des patients ont été opérables avec une bonne évolution après un recul de 3 ans en moyenne.
Conclusion: Conformément à d'autres études, notre travail montre la fréquence de l'adénocarcinome chez le sujet sans habitudes toxiques.

Conflits d'intérêt: Rien à déclarer

\section{P85}

\section{Mésothélium Pleural Malin}

R. Cherkaoui, L. Elhamdaoui, H. Arfaoui, H. Jabri, W. Elkhattabi, H. Afif

Service des Maladies Respiratoires, Hôpital 20 Août 1953, CHU Ibn Rochd

Introduction: L'exposition à l'amiante constitue le principal facteur du mésothéliome pleural malin(MPM). Cette exposition est essentiellement d'origine professionnelle, mais elle peut également être environnementale. Le diagnostic du mésothéliome reste difficile.

Méthode: Le but de notre travail est d'étudier le profil épidémiologique et radiologique. Nous rapportons 43 cas de MPM colligés sur une période de 20 ans.

Résultats: Notre étude concerne 31 hommes (72\%) et 12 femmes (28\%). La moyenne d'âge est de 62 ans. L'exposition à l'amiante est retrouvée dans tous les cas. Elle est professionnelle dans 30 cas $(69,7 \%)$, environnementale et domestique dans 13 cas (30,3\%). La symptomatologie clinique révélatrice est faite de toux sèche dans tous les cas, de douleur thoracique et de dyspnée à l'effort dans 40 cas, l'altération de l'état général est notée chez tous les patients. La radiographie thoracique retrouve une opacité de type pleural dans tous les cas, associée à un aspect mamelonné de la plèvre dans 17 cas et à une rétraction de l'hémithorax dans 6 cas. L'atteinte pleurale est unilatérale dans 40 cas et bilatérale dans 3 cas. Des nodules pulmonaires sont associés chez 11 patients. Le dosage de l'acide hyaluronique dans le liquide pleural fait dans 10 cas est revenu négatif dans 6 cas. L'aspect du liquide à la ponction pleurale est séro- hématique dans 32 cas, jaune citrin dans 10 cas et hématique dans un seul cas. Le cytodiagnostic du liquide a révélé la présence de cellules suspectes de malignité dans 11 cas. Le diagnostic de certitude est porté sur les résultats de la ponction biopsie pleurale dans 32 cas (74\%), par thoracotomie dans 08 cas (19\%), et par ponction biopsie transpariétale dans $7 \%$ des cas. La classification du MPM est dominée par le stade T3 dans 30 cas (70\%). Le traitement préconisé était essentiellement la radiothérapie prophylactique et le traitement symptomatique.

Conclusion: À travers ce travail, on rappelle le rôle de l'exposition professionnelle et environnementale à l'amiante dans la genèse du MPM, les moyens diagnostiques et thérapeutiques de cette tumeur ainsi que son pronostic qui reste péjoratif.

Conflits d'intérêt: Rien à déclarer 


\section{P86 \\ Biomarkers Predictors for Mortality Risk in COVID-19 Patients}

Cristian Cojocaru, Elena Cojocaru

Universitatii 16

Introduction: Severe acute respiratory syndrome coronavirus 2 (SARS-CoV-2) infection can cause a multisystem organ failure, but the factors predicting mortality are not well established. Stratifying risks for severe coronavirus disease (COVID-19) illness could prompt aggressive treatment and early escalation to critical care if appropriate. The aim of the study was to investigate the predictive role of some blood parameters for mortality risk in COVID-19 critical patients in an intensive care unit (ICU).

Method: We performed a retrospective analysis for some biochemical parameters on 59 patients admitted to ICU with COVID-19. Data were collected for deceased versus survived cases on serum ferritin levels, blood glucose, creatinine, lactate dehydrogenase (LDH), International normalized ratio (INR).

Results: The overall mortality rate was $50.8 \%$ with a survival rate after ICU admission ranged from 1 and 35 days. The COVID-19 mortality risk was higher over the age of 60 years $(76.7 \%)$. INR above 1.2 was observed in $60 \%$ of deceased cases compared to $20.7 \%$ in patients who survived $(\mathrm{p}=0.005)$. The significant differences were observed for mean LDH value $(771,8 \pm 426 \mathrm{U} / \mathrm{L}$ vs 380.1 $\pm 192 \mathrm{U} / \mathrm{L}, \mathrm{p}=0.001)$ and serum glucose $(205 \pm 116 \mathrm{mg} / \mathrm{dL}$ vs 136.6 $\pm 41.3 \mathrm{mg} / \mathrm{dL}, \mathrm{p}<0.001)$. The serum creatinine and ferritin were higher in patients who died compared to those who had a favorable evolution but was not reach the significant value. Although, $93.3 \%$ of dead patients had a ferritin level over $400 \mathrm{mg} / \mathrm{dL}$ compared to survivors $(69 \%), \mathrm{p}=0.0392$.

Conclusion: Determination of serum LDH, INR, ferritin and glucose as predictive biomarkers of mortality risk for critically ill patients with COVID-19 disease, in association with male gender and older age (over 60) should be considered in intensive care units.

Disclosure: Nothing to disclose

\section{P87 \\ Santé Respiratoire et Allergique à Madagascar: Impact de la Biomasse}

E. H. Ouaalaya ${ }^{1}$, D. Randriamanana ${ }^{2}$, J. Rakotomizao ${ }^{2}$, R. Raharimanana ${ }^{3}$, J. Rakotoson ${ }^{2}$, M. Tiaray Harison ${ }^{2}$, K. Ravahatra' , J. Rajaoarifetra ${ }^{4}$, J-CA. Rakotoarisoa ${ }^{2}$, G. Rasamimanana ${ }^{3}$, C. Randriamananjara ${ }^{2}$, B. Ravalison ${ }^{2}$, A. Andrianarisoa ${ }^{2}$, A. Ralison ${ }^{3}$, R. Andriamihaja ${ }^{4}$, D. Charpin ${ }^{5}$, P. L'her ${ }^{6}$, C. Raherison-Semjen ${ }^{1,7}$

'Université de Bordeaux, INSERM, Bordeaux Population Health Research Center, équipe: EPICENE, UMR1219, Bordeaux, France, ${ }^{2}$ Université d'Antananarivo, Madagascar, ${ }^{3}$ Université de Mahajanga, Madagascar, ${ }^{4}$ Université de Toamasina, Madagascar, ${ }^{5}$ Université d'Aix-Marseille, Marseille, France, ${ }^{6}$ SPI (Soutien Pneumologie International), Paris, France, ${ }^{7}$ Service des Maladies Respiratoires, Pessac, France

Introduction: A Madagascar, le recours à la biomasse est très répandu. Cependant, il n'existe actuellement aucune donnée épidémiologique disponible à Madagascar sur l'impact sanitaire de l'exposition à la biomasse. En plus, les équations de prédiction malgaches de la spirométrie n'existent pas. Les objectifs de notre étude sont d'étudier l'impact de l'exposition à la biomasse sur la santé respiratoire et d'établir des équations de prédiction pour des adultes malgaches, puis de comparer les mesures malgaches avec les équations de prédiction publiées.

Méthode: Étude descriptive transversale, sur questionnaire, menée chez la population urbaine et rurale de Madagascar entre Janvier 2016 et Janvier 2017. Les variables suivantes ont été recueillies: âge, sexe, taille, le nombre d'heures passées en cuisine, les symptômes respiratoires et des données spirométriques. Le modèle linéaire multiple a été implémenté pour estimer la médiane des données spirométriques (VEMS, CVF, VEMS/CVF). Nous avons comparé les mesures malgaches avec les prévisions de la CECA (European Coal and Steel Community), NHANES III (Third National Health and Nutrition Examination Survey) et l'étude GLI 2012 (ERS Global Lung Function Initiative). Nous avons implémenté GAMLSS afin de modéliser à la fois l'asymétrie et l'aplatissement. Les $\mathrm{z}$-scores et la limite inférieure de la normale ont été calculés.

Résultats: 1004 sujets (72,4\% femmes) âgé de 15 à 95 ans ont été inclus dans notre étude. Parmi eux, 722 sujets ont réalisé la spirométrie. La prévalence des symptômes respiratoires est de $12 \%$; $43,6 \%$ présentant une dyspnée d'effort, $14,7 \%$ une toux chronique et $16,8 \%$ des sifflements dans la poitrine; $36,9 \%$ ont un tabagisme actif, $99 \%$ utilisent le charbon et le bois comme combustible. Il existait une relation significative entre exposition à la biomasse et symptômes respiratoires. Les équations de prédictions du VEMS et de la CVF obtenues par l'approche de GLI 2012 sont les plus proches de notre échantillon avec le plus de précision par les équations des hommes noirs et des femmes d'Asie du sud-Est. Le VEMS et le CVF prédits par la CECA et NHANES III étaient mal appropriés aux mesures malgaches, mais le rapport VEMS/CVF prédit par la CECA est le meilleur. 
Conclusion: La fumée de la combustion de la biomasse expose à des complications pulmonaires. Nos équations de prédiction sont meilleures que celles des études GLI 2012, NHANES III, ECSC en comparant les z-scores.

Conflits d'intérêt: Rien à déclarer

\section{P88 \\ Evaluation du Pronostic des Cancers Broncho-Pulmonaires Primitifs (CBP) Du Sujet Jeune: Étude Comparative Portant sur 70 Patients Âgés de 45 Ans et Moins}

\section{F. Ziane, K. Tlili, R. Belala, A. Meridj, Y. Djeghr. A. Zitouni}

Service de pneumologie. Hôpital militaire régional universitaire de Constantine. Algérie

Introduction: Bien que l'incidence du cancer bronchopulmonaire primitif (CBP)du poumon chez les jeunes adultes reste faible comparée aux âges plus élevés, ce cancer constitue néanmoins un problème important de santé publique.

Méthode: Il s'agit d'une étude prospective et descriptive, comparant deux groupes de patients; un groupe de sujets jeunes (45 ans et moins) inclus par un recrutement séquentiel et un autre groupe de sujets âgés (65 ans et plus) tirés au sort. Le diagnostic de CBP est basé uniquement sur l'étude anatomopathologique. L'exploitation finale et l'analyse des données ont fait appel aux logiciels ÉPI-DATA analysis, ÉPI-INFO 6 et SPSS Statistics 21. Le test de Log Rank est utilisé pour comparer les moyennes des courbes de survie. L'objectif ultime de cette étude est d'avaler le pronostic global des patients jeunes atteints de CBP.

Résultats: Au total, 70 patients jeunes âgés de 45 ans et moins ont été recrutés entre juin 2016 et décembre 2017, lesquels ont été comparés avec un groupe de patients âgés de 65 ans et plus tirés au sort, parmi eux, 24,2\% étaient des femmes. L'adénocarcinome est le type histologique le plus souvent diagnostiqué dans les deux groupes de patients. Seulement $8,6 \%$ sujets jeunes, présentent des comorbidités au moment du diagnostic contre $50 \%$ dans le groupe des sujets âgés $(p=0,01) .94 \%$ des sujets jeunes et $80 \%$ des sujets âgés ont un état général conservé avec un PS entre 0 et 1 . Plus de $84 \%$ des sujets jeunes et plus de $83 \%$ des sujets âgés de 65 ans et plus sont diagnostiqués aux stades localement avancés et aux stades métastatiques. La meilleure réponse au traitement est enregistrée dans le groupe des sujets jeunes; une réponse objective est constatée dans $60 \%$ des cas ( $60 \%$ vs $43,49 \%$ ), La progression tumorale est significativement plus fréquente chez les sujets âgés $(37,67 \%$ vs $12,86 \%$ avec $\mathrm{P}=0,001)$. La survie moyenne des patients jeunes est légèrement meilleure que celle des sujets âgés $(28,721$ mois vs 26,429 mois), mais sur le plan statistique, le test de Log Rank n'a pas mis en évidence une différence significative entre les deux groupes de patients (log Rank=0,1).

Conclusion: Malgréla conservation de l'état général des patients au moment du diagnostic, la rareté des ATCD et des comorbidités associées chez ces patients et la bonne tolérance des traitements, essentiellement la chimiothérapie, le pronostic global n'est pas meilleur par rapport à celui des sujets d'âges avancés. Le stade TNM et le PS restent les principaux facteurs pronostics chez les patients atteints de cancer broncho-pulmonaire primitif.

Conflits d'intérêt: Rien à déclarer

\section{P89 \\ Tuberculose Multifocale Chez L'immunocompétent: À Propos de 71 Cas}

\section{Farissi, N. Zaghba, H. Benjelloun, N. Yassine}

Service de maladies respiratoires $\mathrm{CHU}$ Ibn Rochd Casablanca

Introduction: La tuberculose constitue un problème majeur de santé publique dans le monde et au Maroc. La tuberculose multifocale est définie par l'atteinte de deux sites extrapulmonaires non contigus associée ou non à une atteinte pulmonaire.

Méthode: Nous rapportons une étude rétrospective portant sur 71 cas de tuberculose multifocale chez des patients immunocompétents, colligés au service des maladies respiratoires du CHU IbnRochd de Casablanca sur une période de 18 ans allant de 2000 à 2019.

Résultats: Il s'agisait de 47 femmes et 24 hommes. La moyenne d'âge était de 33 ans. Le délai moyen de consultation était de 72 jours. Tous nos patients étaient vaccinés par le BCG. Un contage tuberculeux récent était retrouvé dans $34 \%$ des cas. Un bilan du terrain réalisé chez tous les patients à la recherche d'une immunodépression était négatif. Nos patients avaient au moins trois atteintes associées. La tuberculose pulmonaire était retrouvée dans tous les cas. Elle était associée à une atteinte pleurale dans 39,2\% des cas, ganglionnaire médiastinale dans $24 \%$ des cas, péritonéale dans $19,4 \%$ des cas, ganglionnaire périphérique dans $16,4 \%$ des cas, splénique dans $14,8 \%$ des cas, un abcès froid de la paroi thoracique dans $12 \%$ des cas, une atteinte uro-génitale dans 10,4\% des cas, péricardique et cérébroméningée dans $8,9 \%$ des cas chacune et ostéoarticulaire dans $7,4 \%$ des cas. Les patients étaient traités selon les recommandations du programme national de lutte contre la tuberculose au Maroc. L'évolution sous traitement antibacillaire était favorable dans $68,9 \%$ des cas. Une patiente avait présenté une rechute tuberculeuse pulmonaire deux années après la 1re atteinte. Nous avions déploré deux décès l'un dans un tableau de détresse respiratoire secondaire à un pneumothorax compliquant une miliaire tuberculeuse, l'autre suite à une défaillance multiviscérale secondaire à une CIVD et 19 patients ont été perdus de vue.

Conclusion: La tuberculose multifocale est souvent décrite comme étant l'apanage de l'immunodéprimé notamment VIHpositif. Nous insistons à travers ce travail sur la possibilité de retrouver cette forme de tuberculose même chez les sujets immunocompétents. Un traitement antituberculeux doit être instauré précocement pour éviter des complications parfois graves.

Conflits d'intérêt: Rien à déclarer 


\section{P90 \\ Particularités Cliniques, Radiologiques, Histologiques et Évolutives du Carcinome Bronchogénique Chez la Femme}

\section{Farissi, H. Benjelloun, N. Zaghba, N. Yassine}

CHU Ibn Rochd, Maroc

Introduction: Le cancer bronchique primitif chez la femme représente le troisième cancer par sa fréquence, derrière le cancer du sein et le cancer colorectal. L'incidence et la mortalité de ce cancer est toujours en augmentation.

Méthode: Le but de ce travail est de souligner les particularités cliniques, radiologiques, histologiques et évolutives du carcinome bronchogénique chez la femme, à travers une étude rétrospective portant sur 102 cas colligés au service des maladies respiratoires du CHU Ibn Rochd entre janvier 2015 et juin 2019.

Résultats: La moyenne d'âge était de 63 ans, avec des extrêmes allant de 38 à 88 ans. Soixante-quatorze pourcent des femmes habitaient en milieu rural, 65\% étaient exposées à la fumée du bois, $18 \%$ étaient exposées à l'amiante, $12 \%$ étaient exposées aux isocyanates, et $14 \%$ étaient des tabagiques actives. Le tabagisme passif était retrouvé chez $18 \%$ des femmes. Le délai moyen de consultation était de 60 jours. La symptomatologie était dominée par la douleur thoracique (80\%), la dyspnée (81\%), le syndrome bronchique (75\%) et l'hémoptysie (38\%). L'examen clinique avait retrouvé un syndrome d'épanchement liquidien dans $38 \%$ des cas, des adénopathies périphériques dans $9 \%$ des cas, alors qu'il était normal dans $53 \%$ des cas. Le téléthorax avait objectivé une opacité à projection hilaire (58\%), une opacité arrondie parenchymateuse (32\%), une opacité de type pleural (25\%), et une atélectasie (12\%). La TDM thoracique avait révélé un processus tissulaire d'allure tumorale dans $92 \%$ des cas, associé à des adénopathies mediastinales dans $69 \%$ des cas, une pleurésie dans 31\% des cas et un nodule spiculé isolé chez deux patientes. La bronchoscopie avait montré un bourgeon tumoral (34\%), une infiltration d'allure tumorale $(23 \%)$ et une compression extrinsèque $(3 \%)$. Le diagnostic était obtenu par une biopsie bronchique (50\%), une ponction biopsie pleurale (18\%), une ponction biopsie transpariétale (11\%), une biopsie sous médiastinoscopie et une biopsie sous thoracotomie dans cinq cas chacune, une biopsie osseuse dans deux cas et une biopsie ganglionnaire périphérique dans un cas. Il s'agissait d'un adénocarcinome pulmonaire primitif dans $64 \%$ des cas, un carcinome épidermoide dans $19 \%$ des cas, un carcinome neuroendocrine à petites cellules dans $14 \%$ des cas. La plupart des carcinomes étaient classés stade IV. Parmi les 102 patientes, 72 ont bénéficié d'un traitement palliatif, 18 d'une chimio-radiothérapie, dix sont décédées. Un traitement chirurgical et une immunothérapie étaient indiquées chez deux patientes chacun.

Conclusion: À travers ce travail, nous insistons sur la fréquence élevée de l'exposition à la fumée du bois comme principal facteur carcinogène chez la femme et sur l'adénocarcinome comme principal type histologique et le pronostic lié à un retard diagnostique.

Conflits d'intérêt: Rien à déclarer

\section{P91 \\ Pandémie COVID-19 et le Risque D'épuisement du Cadre Soignant en Service de Pneumologie}

S. Hajjej, S. Aissa, S. Bouafia, W. Benzarti, A. Knaz, A. Abdelghani, A. Garrouche, A. Hayouni, M. Benzarti, I. Gargouri

Service de Pneumologie CHU Farhat Hached de Sousse, Tunisie

Introduction: Face à la progression rapide de la pandémie COVID-19, les professionnels de santé sont en première ligne dans la prise en charge des patients et eux-mêmes exposés au risque de contamination et maintenant d'épuisement. L'objectif de ce travail est d'évaluer la prévalence del'épuisement professionnel, personnel et relationnel chez le cadre soignant en service de pneumologie.

Matériels et méthodes: Une étude rétrospective a été menée au service de Pneumologie du CHU Farhat Hached à Sousse, intéressant le cadre soignant: ils ont tous répondu au questionnaire du Copenhagen burnout inventory (CBI) qui évalue l'épuisement professionnel, personnel et relationnel au cours de la lére vague du covid-19 (la période entre mars 2020 et mai 2020). Ce questionnaire comporte 19 questions cotées de 0 à 4 (7 questions pour l'épuisement personnel, 6 questions pour l'épuisement professionnel et 6 questions pour l'épuisement relationnel). Pour chaque catégories: si le score est supérieur à 17, l'épuisement est certain, entre 13 et 16, la vigilance est requise, et s'il est inférieur à 13, pas d'inquiétude. A noter que durant cette période, le service à hospitalisé, en unité d'isolement, 59 patients adressés par le biais des urgences pour suspicion de COVID-19 et que tout le cadre soignant a eu une formation concernant les précautions à prendre face à cette pandémie.

Résultats: Parmi le cadre soignant, 38 ont rempli le questionnaire ( $50 \%$ cadre médical et $50 \%$ cadre paramédical), avec un taux de participation à $71 \%$. L'âge moyen était de 35,05 ans $\pm 10,57$ avec des extrêmes entre 24 ans et 65 ans. Le sex-ratio était à 0,35 (10 hommes contre 28 femmes). En moyenne, le nombre des années d'expérience professionnelle était à 10 ans $\pm 9,84$ avec des extrémités allant de 1 an à 35 ans. Un travail posté a été noté dans $39 \%$ des cas. $52,6 \%$ étaient mariés et $68,4 \%$ vivaient en famille. $92,1 \%$ ont un niveau d'étude universitaire. Seulement 4 participants étaient tabagiques (tous des hommes), 18,4\% avaient des maladies chroniques et $7,9 \%$ avaient des antécédents psychiatriques auparavant (dépression). 57,9\% avaient en leur compagnie soit un sujet âgé de plus de 65 ans, soit un sujet avec des maladies chroniques ou une immunodépression. L'épuisement personnel a été noté dans 12 cas $(31,6 \%)$ et il a été corrélé au sexe féminin $(\mathrm{p}=0,012)$, ceux qui étaient entourés par un sujet âgé de plus de 65 ans, soit un sujet avec des maladies chroniques ou une immunodépression $(\mathrm{p}=0.031)$ et ceux ayant des antécédents psychiatriques $(\mathrm{p}=0,008)$. L'épuisement professionnel a été noté aussi dans 12 cas $(31,6 \%)$ et il a été corrélé au sexe féminin $(p=0,034)$, et ceux ayant des antécédents psychiatriques $(\mathrm{p}=0,0029)$. L'épuisement relationnel a été noté dans 8 cas $(21,1 \%)$ et il a été corrélé au sexe féminin $(\mathrm{p}=0,002)$, ceux ayant des antécédents psychiatriques $(p=0,0029)$ et ceux qui rapportent une insomnie $(\mathrm{p}=0,008)$. 6 personnels ont présenté les 3 types d'épuisement simultanément. 
Conclusion: L'épuisement professionnel, personnel et relationnel sont des facteurs majeurs auxquels peut être exposé le cadre soignant durant leur exercice professionnel surtout en période de pandémie. Les repérer et puis les prendre en charge permettra certainement d'améliorer leur qualité de vie et par conséquence leur prestation professionnelle.

Conflits d'intérêt: Rien à déclarer

\section{P92 \\ Degré de l'adaptation du cadre soignant en Service de pneumologie face à la pandémie COVID-19}

\section{S. Hajjej, S. Aissa, S. Bouafia, W. Benzarti, A. Knaz, A. Abdelghani, A. Garrouche, A. Hayouni, M. Benzarti, I. Gargouri}

Service de Pneumologie CHU Farhat Hached de Sousse, Tunisie

Introduction - Objectifs: La pandémie Covid-19, par sa rapidité de diffusion et sa sévérité, et avec les connaissances incertaines, a bouleversé l'environnement professionnel et familial des professionnels de santé. L'objectif de ce travail est d'évaluer le degré de l'adaptation du cadre soignant en service de pneumologie face à la pandémie COVID-19

Matériels et méthodes: Une étude rétrospective a été menée au service de Pneumologie du CHU Farhat Hached à Sousse, intéressant le cadre soignant: ils ont tous répondu au questionnaire: Brief cope score qui évalue le degré d'adaptation du cadre soignant au cours de la lére vague du covid-19 (la période entre mars 2020 et mai 2020). Ce questionnaire comporte 28 questions, cotées de 1 à 4 , et permettant d'évaluer 14 dimensions distinctes du coping. Afin de faciliter la lecture des résultats, c'est la structure à 4 dimensions que nous avons retenue: soutien social (8 items), résolution de problèmes (4 items), évitement (10 items), et pensée positive ( 6 items). A noter que durant cette période, le service à hospitalisé, en unité d'isolement, 59 patients adressés par le biais des urgences pour suspicion de COVID-19 et que tout le cadre soignant a eu une formation concernant les précautions à prendre face à cette pandémie.

Résultats: Parmi le cadre soignant, 38 ont rempli le questionnaire ( $50 \%$ cadre médical et $50 \%$ cadre paramédical), avec un taux de participation à $71 \%$. L'âge moyen était de 35,05 ans $\pm 10,57$ avec des extrêmes entre 24 ans et 65 ans. Le sex-ratio était à 0,35 (10 hommes contre 28 femmes). En moyenne, le nombre des années d'expérience professionnelle était à 10 ans $\pm 9,84$ avec des extrémités allant de 1 an à 35 ans. Un travail posté a été noté dans $39 \%$ des cas. $52,6 \%$ étaient mariés et $68,4 \%$ vivaient en famille. $92,1 \%$ ont un niveau d'étude universitaire. Seulement 4 participants étaient tabagiques (tous des hommes), 18,4\% avaient des maladies chroniques et $7,9 \%$ avaient des antécédents psychiatriques auparavant (dépression). 57,9\% avaient en leur compagnie soit un sujet âgé de plus de 65 ans, soit un sujet avec des maladies chroniques ou une immunodépression. 15 sujets ont augmenté leur consommation du café, et aucun sujet ne prenait des substances psychoaddictives. 14 sujets ont augmenté leurs pratiques religieuses et 4 seulement ont augmenté leurs pratiques sportives. Un score élevé en matière de soutien social a été noté dans 26 cas contre seulement 2 mauvais scores. Pour l'item " résolution des problèmes ", 22 scores élevés contre 5 mauvais scores. Concernant l'approche de l'évitement, on a objectivé seulement 5 mauvais scores, contre 8 mauvais scores pour l'item « pensées positives».

Conclusion: Face à une telle pandémie dont la durée et les conséquences sont assez imprévisibles, les personnels de santé, surtout ceux qui sont en lére ligne, doivent s'adapter à ce nouveau contexte, afin d'éviter un épuisement professionnel et relationnel.

Conflits d'intérêt: Rien à déclarer

\section{P93 \\ Evaluation de L'adhérence des Patients Asthmatiques au Traitement Inhalé: Une Autre Alternative}

\section{S. Hajjej, S. Aissa, R. Handous, A. Omrane, W. Benzarti, A. Abdelghani, A. Garrouche, A. Hayouni ${ }^{2}$, M. Benzarti, I. Gargouri}

Service de Pneumologie CHU Farhat Hached de Sousse, Tunisie

Introduction: La non-observance au traitement inhalé est fréquente chez les patients asthmatiques en Tunisie et a un effet négatif sur le contrôle de la maladie, la morbi-mortalité, ainsi que sur l'économie de la santé. L'objectif de cette étude était d'évaluer l'adhérence des patients tunisiens asthmatiques au traitement inhalé et d'étudier ses retentissements cliniques et spirométriques.

Matériels et méthodes: Nous avons mené une étude descriptive et prospective incluant 54 patients, suivis entre janvier 2018 et décembre 2020 pour asthme et qui avaient tous répondu au questionnaire de Morisky d'adhésion aux médicaments (MMAS-8) à 8 items.

Résultats: L'âge moyen était de 43,09 ans [21-72] avec un sexratio de $0,58.46,3 \%$ des patients avaient un bon contrôle de leur maladie asthmatique, $31,5 \%$ étaient partiellement contrôlés et 22,2\% avaient un mauvais contrôle. Tous les patients étaient sous une corticothérapie inhalée (CSI) (en monothérapie dans 38,8\% des cas et en association avec un bêta- 2 mimétique de longue durée d'action (LABA) dans 61,2\% des cas). Le score MMAS-8 moyen était de 5,13 [1-8]. On a objectivé qu'il est plus élevé chez les fumeurs $(\mathrm{p}=0,019)$ et les patients ayant un asthme allergique $(\mathrm{p}=0,033)$. Un score élevé était associé à plus d'exacerbations $(\mathrm{p}=0.009)$ et plus d'admissions à l'hôpital au cours des 12 derniers mois $(\mathrm{p}=0.028)$. Les antécédents d'hospitalisation en service de réanimation pour asthme aigue grave ont été corrélés à une bonne observance du traitement, mais cela n'était pas statistiquement significatif $(\mathrm{p}=0,071)$. Enfin, la capacité vitale forcée (CVF) ou le volume expiratoire maximal par seconde (VEMS) ne sont pas associés à une meilleure observance.

Conclusion: L'un des principaux défis dans la prise en charge de l'asthme est l'observance au traitement de fond. Le MMAS-8 est un questionnaire bref, validé et reproductible et il peut aider les cliniciens à améliorer la qualité des soins.

Conflits d'intérêt: Rien à déclarer 


\section{P94 \\ Évaluation de la Qualité de vie des Patients Tunisiens Atteints de Dilatation des Bronches}

\section{S. Hajjej, S. Aissa, R. Handous, A. Omrane, W. Benzarti, A. Abdelghani, A. Garrouche, A. Hayouni, M. Benzarti, I. Gargouri}

Service de Pneumologie CHU Farhat Hached de Sousse, Tunisie

Introduction: Les dilatations des bronches (DDB) est une maladie chronique, qui retentit sur la qualité de vie du patient (QDV). Par conséquent, la perception qu'a le patient de son état est très importante pour évaluer l'efficacité du traitement et améliorer la prise en charge. L'objectif de cette étude était d'évaluer la qualité de vie liée des patients atteints des dilatations des bronches en utilisant le Bronchiectasis Health Questionnaire (BHQ).

Matériels et méthodes: Nous avons mené une étude prospective et descriptive incluant 53 patients suivis entre janvier 2017 et décembre 2020 pour des DDB et ayant tous rempli le BHQ. Les patients ayant rapporté une exacerbation de leur maladie au cours des deux semaines précédentes ont été exclus.

Resultats: L'âge moyen était de 53,42 ans [17-80] avec un sexratio de 0,39. 14 patients étaient fumeurs $(26,4 \%)$, avec une consommation moyenne de 44,14 paquets-années. Les DDB étaient localisées seulement chez 12 patients et des DDB cylindriques chez 35 patients, kystiques chez 10 patients et l'association des deux types chez 8 patients. Une étiologie de la bronchectasie a été déterminée chez $51 \%$ des patients. Le symptôme le plus fréquent était une toux productive chez $51 \%$ des patients, puis une hémoptysie chez $30 \%$.

Le score BHQ moyen était de 39,98 [22-60]. Une QDV altérée était corrélée à des DDB diffuse $(\mathrm{p}=0,043)$, kystiques $(\mathrm{p}=0,021)$ ou bien en cas de colonisation bactérienne $(p=0,024)$. Les exacerbations aigues et les admissions à l'hôpital au cours des 12 derniers mois ont été plus fréquemment retrouvées chez les patients avec un score BHQ plus mauvais. Enfin, on a objectivé une corrélation significative entre un mauvais score BHQ et la capacité vitale forcée $(\mathrm{CVF})(\mathrm{p}=0,005)$, mais pas avec le Volume expiratoire maximal par seconde (VEMS) $(\mathrm{p}=0,078)$.

Conclusion: Le BHQ est un questionnaire bref, valide et répétable qui peut aider les cliniciens à améliorer la communication avec les patients atteints des DDB et leur prise en charge.

Conflits d'intérêt: Rien à déclarer

\section{P95 \\ Le Profil Étiologique des Exacerbations de Pneumopathies Infiltrantes Diffuses}

\section{H. Harraz, N. Zaghba, H. Benjelloune, N. Yassine}

Service de maladies respiratoires CHU Ibn Rochd Casablanca

Introduction: L'exacerbation aiguë des pneumopathies infiltrantes diffuses (PID) est caractérisée par l'aggravation aiguë de la fonction respiratoire, pouvant engager le pronostic vital.
Méthode: Une étude rétrospective a été menée au service des maladies respiratoires du CHU Ibn Rochd de Casablanca, incluant 142 patients hospitalisés pour une exacerbation de janvier 2017 au mois de décembre 2019. L'objectif était de décrire les aspects cliniques, paracliniques, étiologiques, et évolutifs des exacerbations de ces PID.

Résultats: Parmi 220 patients hospitalisés pour une PID, 142 exacerbations ont été colligées. Il s'agissait de 57 hommes et 85 femmes, dont la moyenne d'âge était de 58 ans. Les étiologies de ces PID étaient la sarcoïdose ( 98 cas), les connectivités ( 78 cas), la pneumopathie d'hypersensibilité (25 cas), les pneumoconioses (17 cas), la lymphangite carcinomateuse (4 cas), le poumon médicamenteux (4 cas), la fibrose pulmonaire idiopathique (3 cas), post radique ( 3 cas), la lymphangiomyomatose ( 2 cas). Dans 15 cas l'étiologie n'a pas été étiquetée. La symptomatologie clinique était dominée par l'aggravation de la dyspnée de base. L'examen clinique avait noté une fièvre dans 56 cas, un syndrome d'épanchement liquidien dans 10 cas et aérien dans 4 cas. Le bilan radiologique avait objectivé un foyer de pneumonie (66 cas), une pleurésie (10 cas), des images évoquant la tuberculose (13 cas), une aggravation des lésions interstitielles (54 cas) et un pneumothorax (4 cas). Le diagnostic d'accutisation de la maladie a été retenu devant l'aggravation aiguë de la fonction respiratoire et ou l'apparition de nouvelles lésions interstitielles. La cause de l'exacerbation était d'origine infectieuse (70 cas), une décompensation cardiaque gauche (13 cas), une embolie pulmonaire (7 cas), un pneumothorax (4 cas), une tuberculose pulmonaire (13 cas). L'étiologie n'a pas été retrouvée chez 35 patients. L'antibiothérapie était prescrite dans 85 cas associée ou non à une corticothérapie orale de courte durée. L'évolution a été marquée par le décès de 40 patients, et une aggravation fonctionnelle chez 32 patients avec indication de l'oxygénothérapie de longue durée dans 28 cas.

Conclusion: Les exacerbations de PID représentent un facteur de prédictif de mauvais pronostic particulièrement dans la FPI. Les étiologies sont dominées par les causes infectieuses dans notre contexte ce qui rejoint les données de la littérature.

Conflits d'intérêt: Rien à déclarer

\section{P96 \\ Apport de la Biopsie Labiale Dans le Diagnostic de la Sarcoïdose}

Soumaya Debbiche ${ }^{1}$, Imen Sahnoun ${ }^{1}$, Iteb Ben Limem ${ }^{1}$,
Khouloud Chaâbi , Mahdi Abdennadher ${ }^{2}$, Ines Ghanmi',
Sonia Maalej', Leila Douik El Gharbi ${ }^{1}$

${ }^{1}$ Service de pneumologie D, Hôpital Abderrahmen Mami, Ariana, Tunisie, ${ }^{2}$ Service de chirurgie thoracique

et cardiovasculaire, Hôpital Abderrahmen Mami, Ariana, Tunisie

Introduction: la sarcoïdose est une granulomatose systémique, le diagnostic repose sur un faisceau d'arguments dont l'histologie qui met en évidence un granulome tuberculoïde sans nécrose caséeuse. Le but de notre travail est d'évaluer l'apport de la biopsie labiale des glandes salivaires dans le diagnostic de la sarcoïdose.
SSP/SSTS - EFP

Joint Annual Conference 2021 
Méthodes: étude rétrospective de 60 patients suivis au service de pneumologie D de l'hôpital Abderrahmen Mami de l'Ariana durant la période allant de 2001 à 2019.

Résultats: l'âge moyen des patients était de 52,48 ans dont 58 femmes et deux hommes. L'atteinte radiologique était de type I, II, III et IV respectivement chez $10 \%, 63,3 \%, 16,7 \%$ et $10 \%$ des cas. Les atteintes extra thoraciques étaient notées chez 58,3\% des patients: ganglionnaire périphérique $(45 \%)$, hépatique $(18,33 \%)$, splénique $(16,66 \%)$, cutanée $(23,33 \%)$, cavum $(3,3 \%)$, parotide $(6,6 \%)$, ophtalmique (10\%) cérébrale et digestive (chacun 1,6\%). Le diagnostic de la sarcoïdose a été confirmé histologiquement dans $86,6 \%$ des cas et retenu sur un faisceau d'arguments radiocliniques et évolutifs dans $13,4 \%$ des cas. La biopsie labiale des glandes salivaires réalisée chez 40 patients, a montré un aspect de sialadénite lymphocytaire grade 1 , grade 2 , grade 3 et grade 4 de Chisholm et Masson respectivement dans 36,7\%, 6,7\%, 3,3\% et $6,7 \%$ des cas. La biopsie labiale a montré un aspect de granulome non caséeux dans $15,4 \%$ des cas et a étéle seul site biopsié dans $11,5 \%$ des cas.

Conclusion: la biopsie labiale des glandes salivaires, geste peu invasif, est d'un apport non négligeable dans la confirmation de la sarcoïdose médiastino-pulmonaire.

Conflits d'intérêt: Rien à déclarer

\section{P97 \\ Particularités des Pneumopathies Interstitielles Diffuses Chroniques Chez la femme Tunisienne}

\author{
Soumaya Debbiche ${ }^{1}$, Imen Sahnoun ${ }^{1}$, Khouloud Chaâbi ${ }^{1}$, \\ Iteb Ben Limem ', Ines Ghanmi', Salma Mkaddem, Monia Attia², \\ Sonia Maâlej ${ }^{1}$, Leila Douik El Gharbi ${ }^{1}$ \\ ${ }^{1}$ Service de pneumologie D, Hôpital Abderrahmen Mami, \\ Ariana, Tunisie, ${ }^{2}$ Service de radiologie, Hôpital Abderrahmen \\ Mami, Ariana, Tunisie, ${ }^{3}$ Service d'exploration fonctionnelle, \\ Hôpital Abderrahmen Mami, Ariana, Tunisie
}

Introduction: les pneumopathies interstitielles diffuses chroniques (PIDC) constituent un groupe d'affections très hétérogènes et complexes. Le but de notre travail est de déterminer les particularités cliniques, fonctionnelles et radiologiques des PIDC chez la femme.

Méthodes: étude rétrospective de 133 patients suivis pour une PIDC au service de pneumologie D de l'hôpital Abderrahmen Mami de l'Ariana durant la période allant de 2010 à 2019. Les patients ont été répartis en deux groupes: groupe 1(G1): 108 femmes et groupe 2 (G2): 25 hommes.

Résultats: comparés aux PIDC chez l'homme, les PIDC chez la femme se caractérisaient sur le plan clinique par la présence de plus d'arthralgies $(42,6 \%$ vs $12 \%$, p=0,004), de lésions cutanées ( $24 \%$ vs $0 \% ; \mathrm{p}<0,001)$ et sur le plan radiologique par plus d'atteinte micronodulaire $(\mathrm{p}=0,02)$ et réticulo-nodulaire $(\mathrm{p}=0,03)$. Le lavage bronchoalvéolaire a montré un taux de lymphocyte plus important $(\mathrm{p}=0,004)$ et un taux de macrophages plus bas $(\mathrm{p}=0,066)$ dans le G1. La sarcoïdose était l'étiologie la plus fréquente dans le G1 $(32,4 \%$ vs $4 \% ; p=0,004)$. L'intoxication tabagique $(5,6 \%$ vs
$84 \% ; \mathrm{p}<0,001)$, l'exposition professionnelle aux minéraux ( $0 \%$ vs $8 \%$; $<<0,001)$, l'hippocratisme digital $(20,8 \%$ vs $60 \% ; \mathrm{p}<0,001)$ et les lésions d'emphysème $(\mathrm{p}<0,001)$ et de fibrose $(\mathrm{p}=0,008)$ étaient moins fréquentes dans le G1. Les PIDC idiopathiques (36,1\% vs $72 \% ; \mathrm{p}=0,001)$ et notamment la fibrose pulmonaire idiopathique $(22,2 \%$ vs $68 \%$; $\mathrm{p}<0,001)$ étaient moins fréquentes dans le G1. L'âge moyen (59,9 ans vs 65,28 ans; $p=0,07)$, les comorbidités et les symptômes respiratoires étaient comparables entre les 2 groupes. La fréquence des PIDC associées aux connectivites était similaire entre les deux groupes $(17,6 \%$ vs $12 \%$; $\mathrm{p}=0,7)$. Le TM6m a montré plus de désaturation à l'effort dans le $\mathrm{G} 1(\mathrm{p}=0,015)$ et un périmètre de marche comparable entre les 2 groupes ( $314 \mathrm{~m}$ vs $323 \mathrm{~m} ; \mathrm{p}=0,78)$. Les valeurs moyennes de la CVF, CPT, DLCO et de la PaO2 à l'état stable étaient comparables entre les 2 groupes.

Conclusion: les PIDC chez la femme se caractérisent par la fréquence des atteintes extra thoraciques. La sarcoïdose constitue l'étiologie la plus fréquente des PIDC chez la femme.

Conflits d'intérêt: Rien à déclarer

\section{P98}

\section{Atteinte ORL au Cours de la Sarcoïdose Thoracique}

\section{W. Jalloul, H. Benjelloun, N. Zaghba, N. Yassine}

Service des Maladies Respiratoires du CHU Ibn Rochd;

Casablanca

Introduction: La sarcoïdose est une granulomatose systémique affectant principalement l'appareil respiratoire. L'atteinte nasosinusienne est rare, et peut être révélatrice de la maladie.

Méthode: Il s'agit d'une étude rétrospective portant sur 38 cas d'atteinte ORL sur 132 cas de sarcoïdose thoracique colligés au service des maladies respiratoires; sur une période de 22 ans.

Résultats: Il s'agissait de 32 femmes et 6 hommes dont la moyenne d'âge était de 42 ans (23-60 ans). La découverte était fortuite dans $43 \%$ des cas. La symptomatologie était dominée par la dyspnée et la toux sèche. Une parotidomégalie était associée dans 7 cas, un syndrome sec buccal dans 6 cas, une rhinite croûteuse dans 7 cas. À l'imagerie thoracique, la sarcoïdose était classée stade I dans 8 cas, stade II dans 24 cas et stade III dans 6 cas. À l'examen ORL La nasofibroscopie, réalisée chez tous nos patients, retrouvait des granulations nasales dans 9 cas et un aspect pseudotumoral du cavum dans 2 cas; une muqueuse polyploïde des fosses nasales et une déviation septale dans 2 cas chacune. Les biopsies nasales, réalisées chez tous nos patients, concluaient à une inflammation granulomateuse tuberculoïde sans nécrose caséeuse dans dix cas seulement. La biopsie labiale faite dans 30 cas montrait un granulome épithélio-gigantocellulaire sans nécrose caséeuse dans 20 cas. Le blondeau scanner objectivait une sinusite maxillaire et un comblement des cellules éthmoïdales dans 5 cas. La corticothérapie systémique et locale était indiquée dans la majorité des patients avec une bonne évolution.

Conclusion: Il ressort de ce travail que l'atteinte ORL au cours de la sarcoïdose existe mais reste rare, ce qui rejoint les données de la littérature.

Conflits d'intérêt: Rien à déclarer 


\section{P99}

\section{Hépatotoxicité Aux Antituberculeux}

W. Jalloul, N. Zaghba, H. Benjelloun, N. Yassine

Service des maladies respiratoires du CHU Ibn Rochd, Casablanca

Introduction: La tuberculose demeure un enjeu majeur de santé publique à l'échelle planétaire et dans notre contexte. La toxicité hépatique des antituberculeux est un effet secondaire sérieux qui peut aller d'une simple perturbation transitoire du bilan hépatique jusqu'à l'hépatite fulminante imposant l'arrêt immédiat des anti-bacillaires. Le but de notre travail est de préciser les difficultés de prise en charge thérapeutique de l'hépatotoxicité aux anti-bacillaires.

Méthode: Nous rapportons 19 observations d'hépatite toxique aux anti bacillaires colligées au service des maladies respiratoires du CHU Ibn Rochd; sur une période de 5 ans.

Résultats: Il s'agissait de dix femmes et neuf hommes avec une moyenne d'âge de 39 ans. La tuberculose était pulmonaire dans 16 cas, pleurale dans deux cas et ganglionnaire dans un cas. L'hépatotoxicité était découverte dans 6 cas lors de la surveillance du bilan hépatique, et dans 13 cas à l'occasion de symptômes. Le délai moyen de survenue des signes cliniques ou biologiques d'hépatotoxicité était de 38 jours (2-150 jours). Le bilan hépatique avait montré une hépatite cytolytique dans neuf cas, une hépatite cholestatique dans deux cas et une atteinte mixte dans huit cas. Le traitement anti bacillaire a été arrêté chez tous les patients. La reprise du traitement était progressive en commençant par l'anti bacillaire le moins hépatotoxiques avec une bonne évolution dans presque tous les cas.

Conclusion: L'hépatite toxique aux anti bacillaires est certes rare, mais son apparition menace l'observance thérapeutique et le pronostic de la maladie. Ainsi, la prévention s'impose par le strict respect des règles de prescription, le dépistage précoce des signes d'intolérance thérapeutique et le suivi rigoureux de l'évolution sous traitement anti-bacillaire.

Conflits d'intérêt: Rien à déclarer

\section{P100 \\ Association Tuberculose Carcinome Bronchogénique}

\section{Z. Laklaai, N. Zaghba, H. Benjelloun, N. Yassine}

Service des maladies respiratoires $\mathrm{CHU}$ ibn rochd Casablanca

Introduction: Les relations entre cancer pulmonaire et tuberculose sont complexes. La tuberculose a longtemps été suspectée d'augmenter le risque de cancer pulmonaire tandis que le cancer et ses traitements sont des facteurs susceptibles de favoriser la réactivation d'une tuberculose. L'association de ces deux pathologies chez un même patient est rare et complique la prise en charge et le pronostic.
Méthode: Nous rapportons 12 cas d'association carcinome bronchogénique et tuberculose pulmonaire colligés au service des maladies respiratoires.

Résultats: Les 12 patients étaient de sexe masculin dont la moyenne d'âge était de 52 ans. Dans les antécédents on trouve un tabagisme actif dans tous les cas, une consommation de cannabis chez 4 patients et un diabète chez 3 patients. La symptomatologie clinique était dominée par la dyspnée, la douleur thoracique et l'altération de l'état général. La radiographie thoracique a objectivé une opacité excavée chez quatre patients, un foyer de condensation dans 6 cas et un trouble de ventilation dans 2 cas. Le diagnostic de tuberculose pulmonaire a été retenu sur examen direct des expectorations ou le liquide d'aspiration bronchique dans 7 cas, culture dans le liquide d'aspiration bronchique dans 2 cas et genexpert dans le liquide d'aspiration bronchique chez 3 patients. La bronchoscopie avec biopsies bronchiques a permis de confirmer le diagnostic histologique du carcinome bronchogénique dans 10 cas et par ponction biopsie transpariétale dans 2 cas, le type histologique prédominant était le carcinome épidermoïde. La chimiothérapie a été instaurée après deux mois de traitement anti bacillaire chez 7 patients, des soins palliatifs ont été indiqués chez 3 patients, et 2 patients sont décédés.

Conclusion: La tuberculose active qui complique le cancer du poumon est un problème clinique important dans notre pays. Relativement rare mais grave, cette association pose un problème de diagnostic, de tolérance à la double chimiothérapie antituberculeuse et antimitotique et un problème de pronostic lié au taux de mortalité élevé du carcinome bronchogénique.

Conflits d'intérêt: Rien à déclarer

\section{P101 \\ Hydatidose Multiviscérale}

\section{EL Hamdaoui, W. EL Khattabi, H. Jabri, H. Arfaoui, H. Afif}

Service des Maladies Respiratoires, Hôpital 20 Août 1953, $\mathrm{CHU}$ Ibn Rochd

Introduction: L'hydatidose multiviscérale est une forme rare qui représente 5 à $7,5 \%$ des hydatidoses. Dominée par l'atteinte hépato-pulmonaire, elle peut toucher plusieurs organes. Le traitement essentiellement chirurgical peut poser des problèmes si le kyste est à localisations multiples.

Méthode: Nous avons mené une étude sur une période de huit ans (Janvier 2013 à Décembre 2020) concernant 105 cas d'hydatidose.

Résultats: Nous avons recensé 30 cas d'hydatidose multiple dont la prévalence est de $28.5 \%$. La moyenne d'âge est de 35 ans, le sexe masculin est légèrement prédominant (53\%). Un contage hydatique est retrouvé dans $56 \%$ des cas. Un antécédent de kyste hydatique opéré hépatique dans 5 cas et pulmonaire dans 3 cas. La symptomatologie clinique est représentée essentiellement par la douleur thoracique dans $86 \%$ des cas, un syndrome bronchique dans $73 \%$, des hémoptysies dans 33\% des cas, l'hydatidoptysie dans $19 \%$ des cas. La majorité des patients sont en bon état général. La radiographie thoracique a objectivé une opacité bien limitée dans 
$73 \%$ des cas. Le nombre des kystes hydatiques pulmonaires est en moyenne 3. La TDM thoracique a confirmé la nature kystique des opacités thoraciques et a objectivé d'autre localisations; hépatique dans $43 \%$ des cas, pleurale dans $26 \%$ des cas, médiastinale dans $13 \%$ des cas, splénique dans $7 \%$, et un cas d'hydatidose diaphragmatique, un cas d'hydatidose cardiaque, et un cas d'hydatidose vertébrale, pariétale thoracique et médiastinale. Le traitement médical à base d'albendazole a été le choix de la prise en charge dans $36 \%$ des cas avec une bonne évolution radio-clinique après un recul de 3 ans, le traitement chirurgical suivi d'un traitement médical est préconisé dans $46 \%$ des cas et un traitement chirurgical seul dans $36 \%$ des cas portant d'abord sur l'atteinte pulmonaire puis les autres organes.

Conclusion: L'hydatidose multiple reste moins fréquente mais peut être grave par ses localisations.

Conflits d'intérêt: Rien à déclarer

\section{P102 \\ Apport de la Ponction-Biopsie Transpariétale (PBTP) Echoguidée Dans Les Condensations Pulmonaires Persistantes: Expérience du Service de Pneumologie de l'Hôpital « 20 août 1953 » de Casablanca}

\section{EL Hamdaoui, W. EL Khattabi, H. Jabri, H. Arfaoui, H. Afif \\ Service des Maladies Respiratoires, Hôpital 20 Août 1953, CHU Ibn Rochd}

Introduction: La condensation pulmonaire persistante pose un problème de diagnostic étiologique. Les moyens de diagnostic conventionnels et principalement la bronchoscopie peuvent être insuffisants. Dans ces cas la PBTP échoguidée trouve tout son intérêt.

Méthode: Nous avons mené une étude rétrospective monocentrique descriptive et analytique de janvier 2010 à Décembre 2020.

Résultats: Parmi 346 cas de condensations pulmonaires, 38 étaient persistants dont 17 ont bénéficié d'une PBTP échoguidée après décision collégiale. Ce geste a été réalisé par des pneumologues expérimentés. Elle avait un rendement diagnostique de $76 \%$. L'origine maligne a été retenue dans $70 \%$ des cas majoritairement des adénocacinomes. Une étiologie bénigne a été retrouvée dans 3 cas dont un seul cas de tuberculose. La plupart des patients ont présenté une douleur au site de ponction qui a cédé aux antalgiques du premier pallier. Un seul patient a présenté une hémoptysie de grande abondance nécessitant une prise en charge en réanimation. Il n'y avait aucun cas de pneumothorax iatrogène.

Conclusion: La PBTP échoguidée est une méthode diagnostique efficace qui évite le recours à des gestes plus invasifs avec un rendement diagnostic élevé surtout pour les étiologies malignes.

Conflits d'intérêt: Rien à déclarer

\section{P103 \\ La Chirurgie des Tumeurs Bénignes des Gaines Nerveuses du Thorax}

M. Abdennadher ${ }^{1}$, K. Ben Amara ${ }^{1}$, H. Zribi ${ }^{1}$, I. Bouacida ${ }^{1}$, S. Zairi ${ }^{1}$,
N. Balloumi ${ }^{2}$, Y. Houcine ${ }^{3}$, S. Ouerghi ${ }^{4}$, A. Marghli

${ }^{1}$ Service de chirurgie thoracique et cardiovasculaire. Hôpital Abderrahmane Mami. Ariana, ${ }^{2}$ Service de pneumologie PAV IV. Hôpital Abderrahmane Mami. Ariana, ${ }^{3}$ Service d'anatomopathologie. Hôpital Abderrahmane Mami. Ariana, ${ }^{4}$ Service d'anesthésie réanimation chirurgicale. Hôpital Abderrahmane Mami. Ariana Faculte de Medecine de Tunis. Universite Tunis el Manar

Introduction: Les tumeurs bénignes des gaines nerveuses (TBGN) sont des tumeurs neurogènes rares qui se développent à partir des différents éléments constitutifs du nerf. Le but de notre travail est de décrire les variétés cliniques et paracliniques des TBGN thoraciques ainsi que leur traitement chirurgical et leur pronostic.

Méthode: Il s'agit d'une étude rétrospective réalisée sur une durée de 5 ans allant de janvier 2015 au mois de décembre 2020, incluant 14 patients opérés pour une tumeur thoracique et dont l'examen anatomopathologique définitif conclut à une TBGN.

Résultats: La moyenne d'âge était de 54 ans avec une prédominance féminine de $64 \%$. La douleur thoracique était le principal symptôme (71\%). La radiographie a révélé une opacité médiastinale ovalaire homogène dans $79 \%$ des cas. La tomodensitométrie thoracique, réalisée chez tous les patients a montré une masse médiastinale postérieure ( 9 patients), antérieure ( 2 patients) et une masse tissulaire au niveau du plan musculaire thoracique postérieur. Une IRM réalisée chez 5 patients a montré une extension rachidienne (2cas). Les voies d'abord étaient une thoracotomie postéro-latérale ( 9 patients), une mini-thoracotomie vidéo-assistée (2 patients), une thoracotomie latérale (2 patients) et une incision élective (1patient). Une exérèse tumorale complète a été réalisée chez tous les patients. Le drainage en post-opératoire avait une durée moyenne de 3 jours. Les suites opératoires étaient simples pour tous les patients. L'étude histologique avait montré 10 cas de schwannome et 4 cas de neurofibrome. Aucune récidive ni transformation maligne n'ont été notées avec un recul de deux ans.

Conclusion: Les TBGN sont des tumeurs rares de l'adulte. La principale localisation est le plus souvent intra-thoracique au niveau du médiastin postérieur. L'exérèse totale est le seul garant d'une guérison sans récidive. Cependant, un suivi prolongé et régulier est nécessaire du fait de la possibilité de récidive après chirurgie.

Conflits d'intérêt: Rien à déclarer 


\section{P104 \\ Corticosteroids for Covid-19: The Search for an Optimum Timing of Therapy}

\section{Ferchichi, B. Hamdi, S. Louhaichi, N. Boubaker, I. Khalfallah, S. Belkhir, J. Ammar, A. Hamzaoui}

Pneumologie B de I'hôpital Abderrahmen Mami

Introduction: In the current SARS-CoV-2 pandemic, there has been worldwide debate on the use of corticosteroids in COVID-19. In the RECOVERY trial, dexamethasone has reduced 28-day mortality in patients requiring oxygen therapy. However, the optimum timing to start corticosteroid remain uncertain, and reliable data are needed.

Aim: To evaluate the impact of the timing of initiation corticosteroids on moderate to severe Covid-19 disease's outcomes.

Methods: Retrospective study conducted from October 2020 to January 2021 in the pulmonary departement B of Abderrahmen Mami hospital on confirmed cases of COVID-19. We compared the outcomes of early used corticosteroids ( 7 days from symptoms onset) to lately used corticosteroids (after 7 days of symptoms onset) in Covid-19 patients requiring supplemental oxygen.

Results: One hundred ninety six cases of COVID-19 patients were included in our study. The median age was $61,32 \pm 2,5$ years. The sexe-ratio was 1,8 . The median time to symptoms onset on admission was 8 days. Patients treated with corticosteroids early $(\mathrm{n}=74)$ had lower CRP $(\mathrm{p}=0.04)$, higher fibrogen $(\mathrm{p}=0,02)$, higher DDimer $(\mathrm{p}=0,002)$ and more lymphopenia $(\mathrm{p}=0.05)$ compared to patients treated with corticosteroide lately $(n=101)$. Introducing corticosteroid therapy in the first week of symptoms was associated with an increase in the time to recovery $(p=0,05)$, a higher ICU admission rate $(\mathrm{p}=0,001)$ and a higher risk of mechanical ventillation $(p=0,002)$. Mortality and thromboembolic events were similar in the 2 groups.

Conclusion: Corticosteroids seem to be a double-edged sword in the fight against COVID-19. More studies are needed to define the optimum time to introduce it.

Disclosure: Nothing to disclose

\section{P105 \\ Predictive Level of D-Dimer on Admission in Patients with Covid-19?}

\section{Ferchichi, S. Louhaichi, I. khalfallah, S. Belkhir, \\ N. Boubaker, J. Ammar, B. Hamdi, A. Hamzaoui}

Pneumologie B de I'hôpital Abderrahmen Mami

Introduction: $\mathrm{D}$-dimer elevation has been reported to be one of the common laboratory findings noted in Covid-19 patients. However, the optimal cutoff for D-dimer on admission to predict venous thromboembolism (VTE) in patients with COVID-19 has not been well evaluated.

Our study aimes to evaluate the D-dimer cut-off value to predicting VTE in COVID-19 patients.
Méthods: Patients with laboratory confirmed Covid-19 were retrospective enrolled in the pulmonary departement B at Abderrahmen Mami hospital, Tunisia, from March 2020 to January 2021. D-dimer levels on admission and venous thrombotic events were collected to calculate the optimum cutoff using receiver operating characteristic curves. According to the cutoff, the subjects were divided into two groups.

Results: A total of 200 patients were enrolled in our study, with a median age of 64,9 [22-98] years. The incidence of VTE was $15 \%$ $(30 / 200)$ of which $16 \%$ occured on thrombosis prophylaxis. Pulmonary embolism occured in 21 patients and deep venous thrombosis in 9 patients. The optimum cutoff value of D-dimer to predict VTE was $1.4 \mu \mathrm{g} / \mathrm{ml}$ using ROC curve with a sensitivity of $82.3 \%$ and a specificity of $85.3 \%$. Area under the ROC curve for VTC was 0.89 . There were 81 patients with D-dimer $\geq 1,4 \mu \mathrm{g} / \mathrm{mL}$ and 119 patients with $\mathrm{D}$-dimer $<1,4 \mu \mathrm{g} / \mathrm{m}$ on admission. Patients with $\mathrm{D}$-dimer levels $\geq 1,4 . \mu \mathrm{g} / \mathrm{mL}$ had a higher incidence of VTE $(\mathrm{P}<0.001)$. They also had higher incidence of underlying disease, such as diabetes $(\mathrm{P}=0.007)$ and hypertension $(\mathrm{P}<0.001)$. Lower level of lymphocyte $(\mathrm{P}=0.03)$ and higher level of $\mathrm{C}$-reactive protein $(\mathrm{P}=0.04)$, were also observed in those with D-dimer levels $\geq 1.4 \mu \mathrm{g} / \mathrm{mL}$.

Conclusion: D-dimer on admission greater than $1.4 \mu \mathrm{g} / \mathrm{mL}$ could be an early and helpful marker to prevent VTE in COVID-19 patients.

Disclosure: Nothing to disclose

\section{P106 \\ Risk Factors for Radiological and Functional Sequelae in Multi-Drug Resistant Tuberculosis}

\author{
M. Ferchichi, S. Be Saad, A. Ben Mansour, O. Neffati, S. Belhadj, \\ L. Slim, H. Daghfous, F. Tritar
}

Pneumologie C de l'hôpital Abderrahmen Mami

Introduction: Treatment of multidrug-resistant (MDR-TB) mainly focuses on bacteriological cure. However, only limited studies have evaluated the radiological and fonctional sequelae left at the end of treatment.

Aim: To assess risk factors for radiological sequelae and pulmonary function impairment in MDR-TB patients at the end of treatment.

Methods: A cross-sectional, retrospective study conducted at Abderahman Mami Hospital and Menzel Bourguiba Hospital in Tunisia on 140 patients with MDR-TB treated between 2000 and 2019. Patients who completed MDR-TB treatment or cured, had a Medical Research Council (MRC) dyspnea grade evaluation, chest $\mathrm{X}$-ray (CXR) and pulmonary function testing (PFT) assessment using spirometry.

Results: Eighty-eight patients were enrolled with mean age of $42 \pm 13$ years (27-62). The sex ratio was 3.5 . Smoking and history of prior TB treatment were the most frequent risk factos for MDR-TB. Half of the patients had poor socio-economic condition. Twentythree (26\%) were underweight. The most common medical comorbidity was diabetes mellitus (18\%). The median follow-up time was 41 months. At follow up, $85.7 \%$ of patients complained of residual 
symptoms: breathlessness and cough were the most common symptom with grade II and III dyspnea on MRC scale in $65 \%$ and $31 \%$. On radiological evaluation, 65 patients $(73 \%)$ had residual chest $\mathrm{x}$-ray abnormality with $53 \%$ patients showing bronchiectasis and $23,4 \%$ emphysema, $37 \%$ advanced lesions and $21 \%$ bilateral lesions. PFT was abnormal in $87.6 \%$ patients with mixed pattern being the commonest abnormality. Patients with pre XDR or XDR TB (OR: 3,07; IC 95\% [1,22,14,3]), advanced lesions (OR: 2,24; IC $95 \%[1,13,27,7]$ ) and prior TB treatment (OR: 4,2; IC 95\% $[1,7,27,3])$ were found to have significant radiological sequelae. Linear regression analysis showed that the FEV1 (\% predicted) and the FVC (\% predicted) were negatively correlated to dyspnea grade on MRC scale ( $\mathrm{r}=-0.31,-0.50)$.

Conclusion: The post-treatment pulmonary rehabilitation of MDR-TB patients should be an integral part of DOTS Plus programme particularly in case of prior TB treatment and XDR TB.

Disclosure: Nothing to disclose

\section{P107 \\ Acquired Multidrug-Resistant Tuberculosis versus Primary versus: The Role of Social Determinants}

\section{Ferchichi, S. Be Saad, A. Ben Mansour, O. Neffati, S. Belhadj, L. Slim, H. Daghfous, F. Tritar}

Pneumologie C de l'hôpital Abderrahmen Mami

Background: In tuberculosis, acquired and transmitted resistance arise from two different mechanisms that are associated with different risk factors.

Aim: Identify risk factors associated with acquired (MDR-A) and primary (MDR-P) resistance respectively.

Methods: Retrospective, comparative and multicentre study including patients treated for MDR-TB from 2000 to 2019 in pulmonology departments of Ariana and Menzel Bourguiba hospitals. The characteristics of MDR-A and MDR-P patients were compared.

Results: Our study included 140 patients aged of $42 \pm 13$ years (27-80). The sex ratio was 3.82. Seventy-five percent of patients were illiterate or had primary education. Smoking was the main risk factor for TB (76\%). Diabetes mellitus was the most common comorbidity (24\%). Primary resistance was found in 35 cases (25\%). Subjects with MDR-P were younger ( 31 vs 47 years; $\mathrm{p}=0,04$ ), more educated ( $48 \%$ vs $8 \% ; \mathrm{p}=0,01$ ), less likely to have a history of incarceration ( $4 \%$ vs $67 \%$; $\mathrm{p}=0,001)$, radiological sequelae $(74 \%$ vs $18 \%)$ ), higher socioeconomic status (SES) ( $56 \%$ vs $12 \%$; $=0,04$ ) than those with MDR-A. Poor adherence and treatment failure were significantly higher in MDR-A ( $44 \%, 67 \%$ vs $13 \%, 8 \%$; $=0,05$; $\mathrm{p}=0,02)$. In multivariate analysis, patients of medium and high SES were 3.11 (95\% CI: 0.47-5.24) and 3.75 (95\% CI: 0.91-22.8) times likely to have MDR-P compared to MDR-A.

Conclusion: Lower SES, poor adherence and treatment failure were significantly higher in MDR-A. Thus, health education programs should target this specific subgroup of patient to interrupt MDR-TB transmission.

Disclosure: Nothing to disclose

\section{P108 \\ Particularities of Covid-19 in the Elderly}

Ferchichi Meriam, Khalfallah Ikbel, Nouha Boubaker, Belkhir Safa, Molk ben Bechir, Louhaichi Sabrine, Hamdi Besma, Ammar Jamel, Hamzaoui Agnès

Pneumologie B de l'hôpital Abderrahmen Mami

Introduction: Although the population is generally susceptible to SARSCoV-2, elderly patients have higher morbidity and mortality. Thus, the clinical characteristics and outcomes of elderly and young patients may be different.

Aim: To compare the clinical features of COVID-19 and outcomes in elderly patients to non- elderly (young and mild aged) patients.

Methods: A retrospective study including Patients admitted, between March 2020 and January 2021, in the pulmonary departement B at Abderrahman Mami Hospital (Tunisia), for confirmed severe acute respiratory syndrome coronavirus 2 infection (SARS Cov 2). Clinical, radiological and laboratory findings and clinical outcomes of elderly patients (G1: older than 65 years) were compared to non-elderly patients (G2: younger than 65 years).

Results: A total of 200 patients, 89 elderly (44\%) and 111 younger patients (55\%), were enrolled. The sexe ratio was 4,2 and 2,8 respectively in the 2 groups. The median age in G1 was 68 years while the median age of $\mathrm{G} 2$ was 47 years $(\mathrm{p}<0.001)$. Comorbidities were more common in the elderly group ( $82 \%$ vs $51 \%$; $\mathrm{p}=0,02)$. COVID-19 was severe in $71 \%$ of cases in G1 vs $31 \%$ in youngers $(\mathrm{p}=0,01)$. Digestive manifestations were frequent in elderly patients ( $78 \%$ vs $24 \%$; $\mathrm{p}=0,002$ ) whereas fever was significantly common in G2 (67\% vs $15 \% ; p=0,004)$. The lymphocyte count in $\mathrm{G} 1$ was significantly lower $\left(1200 / \mathrm{mm}^{3}\right.$ vs $\left.400 / \mathrm{mm}^{3} ; \mathrm{p}=0.03\right)$ however the C-reactive protein level was significantly higher than in G2 (245 vs $75 \mathrm{mg} / \mathrm{l} ; \mathrm{P}=0.04)$. The ARDS and acute kidney function injuries were higher in elderly group ( $67 \%$ vs $35 \% \mathrm{p}=0.05 ; 58 \%$ vs $12 \%$ $\mathrm{p}=0.02)$. Mortality at 21 days was higher in elderly group ( $57 \%$ vs $11 \% ; \mathrm{p}=0.01$ ).

Conclusion: COVID-19 appears as a severe condition in the elderly patients with a high 3 -week mortality rate. Distinctive features can be identified in older adults, including atypical clinical presentation.

Disclosure: Nothing to disclose

\section{P109 \\ La Lymphangioleiomyomatose}

N. Bougteb, W. Elkhattabi, S. Raftani, H. Arfaoui, H. Jabri, H. Afif

Service des Maladies Respiratoires, Hôpital 20 Août 1953, CHU Ibn Rochd

Introduction: La lymphanghioleiomyomatose (LAM) est une maladie pulmonaire rare rencontrée quasi exclusivement chez la femme en période d'activité génitale, son association à la sclérose tubéreuse de Bourneville (STB) est fréquente. L'atteinte 
pulmonaire est caractérisée par des kystes pulmonaires multiples, des pneumothorax récidivants et l'évolution vers l'insuffisance respiratoire chronique.

Méthode: Nous avons mené une étude rétrospective de janvier 2005 à janvier 2019 où nous avons enregistré 5 cas de LAM.

Résultats: Il s'agissait de femmes d'une moyenne d'âge moyen de 34 ans. La dyspnée était un signe constant. La douleur thoracique a été révélatrice d'un pneumothorax inaugural dans 4 cas. Les signes extra-respiratoires étaient en faveur de la STB dans 4 cas. Le scanner a objectivé des kystes pulmonaires arrondis à parois fines diffus très évocateurs de la LAM chez les 5 patientes. La TDM abdominale a objectivé 3 cas d'angiolipomes, un cas de lymphadenopathies, un cas de lymphangiome et un cas de lymphomyomes uterins. L'IRM a objectivé des tubers corticaux. Quatre patientes étaient au stade d'insuffisance respiratoire chronique associée à un trouble ventilatoire restrictif ou mixte et une hypertension pulmonaire dans 2 cas. Le diagnostic de LAM-STB a été retenu chez 4 patientes et une une LAM sporadique possible dans un cas. En dehors du traitement symptomatique, quatre patientes ont bénéficié d'un drainage pleural et trois d'un talcage sous thoracoscopie. Trois patientes étaient mises sur la liste d'attente de transplantation pulmonaire. Une patiente avait un traitement par inhibiteurs de la M-TOR. Deux patientes sont décédées d'insuffisance respiratoire.

Conclusion: Notre étude souligne la gravité de la LAM qui souffre du sous-diagnostic et de limites thérapeutiques dans notre contexte.

Conflits d'intérêt: Rien à déclarer

\section{P110 \\ Intérêt du Test de Contrôle de l'asthme (ACT) Dans la Prise en Charge des Patients Asthmatiques à Casablanca}

H. Arfaoui, S. Aidou, H. Jabri, W. Elkhattabi, H. Afif

Service des Maladies Respiratoires, Hôpital 20 Août 1953, CHU Ibn Rochd

Introduction: Quelle que soit la sévérité de l'asthme, le contrôle de la maladie asthmatique est l'objectif thérapeutique principal L'intérêt de notre étude est d'évaluer, grâce au test de contrôle de l'asthme, les facteurs prédictifs d'un mauvais contrôle de l'asthme dans notre population casablancaise.

Méthode: Il s'agit d'une étude prospective, portant sur 185 dossiers de patients asthmatiques suivis en consultation de pneumo-allergologie de l'hôpital 20 Août de Casablanca, ayant adhéré au questionnaire du test de contrôle de l'asthme. La version validée française de l'ACT a été traduite en dialecte arabe.

Résultats: L'âge des patients se situait entre 19 et 51 ans avec un âge moyen de 35 ans. Nous avons noté une nette prédominance du sexe féminin dans cet échantillon, les femmes représentent $64 \%$ des patients contre $36 \%$ d'homme. Soixante-cinq pour cent des patients étaient issus d'un niveau socio-économique bas dont $50 \%$ étaient issus du milieu rural et $50 \%$ habitaient dans des bidonvilles, $35 \%$ des patients étaient d'un niveau socio-économique moyen. L'atopie personnelle était retrouvée chez $24,7 \%$ des patients. Le tabagisme actif est retrouvé dans $21 \%$ des cas. L'obésité est retrouvée chez 51 patients ( $27 \%$ des cas). Selon l'ACT, l'asthme est totalement contrôlé dans $45 \%$ des cas, partiellement à non contrôlé chez $55 \%$ des patients.

Conclusion: Nous avons conclu à travers notre étude statistique qu'un bas niveau socio-économique est le seul paramètre statistiquement significatif. Or, nous n'avons pas trouvé une corrélation statistique du contrôle de l'asthme avec l'âge, le sexe, le terrain d'atopie, l'obésité ou le tabagisme

Conflits d'intérêt: Rien à déclarer

\section{P111 \\ Profil clinique et étiologique du Poumon éosinophile}

S. Aidou, H. El kihal, H. Arfaoui, H. Jabri, W. Elkhattabi, H. Afif

Service des Maladies Respiratoires, Hôpital 20 Août 1953, $\mathrm{CHU}$ Ibn Rochd

Introduction: Le poumon éosinophile est un groupe hétérogène d'affections caractérisées par une infiltration du parenchyme pulmonaire par les éosinophiles, associée ou non à une éosinophilie circulante. L'objectif de l'étude était de relever les caractéristiques cliniques et paracliniques du poumon éosinophile et de dégager le profil étiologique de ce groupe d'affections.

Méthode: Il s'agit d'une étude rétrospective incluant 9 patients (8 femmes et un homme) hospitalisés pour un poumon éosinophile entre 2011 et 2020 .

Résultats: L'âge moyen était de 36 ans, des antécédents d'asthme ont été retrouvés chez tous les patients. Les signes cliniques étaient dominés par la toux et la dyspnée retrouvés chez tous les patients. Une atteinte neurologique dans 4 cas, une péricardite et une pleurésie bilatérale dans 2 cas, une atteinte sinusale dans un cas. L'hyperéosinophilie sanguine était en moyenne de 2730 éléments. L'éosinophilie a été retrouvée dans le lavage broncholoalvéolaire. Les étiologies retrouvées étaient l'angéite de Churg et Strauss (6 cas) et l'aspergillose bronchopulmonaire allergique ( 3 cas). Tous les patients étaient traités par une corticothérapie par voie générale associée à un traitement antifongique et immunosuppresseur dans respectivement un cas. L'évolution était favorable chez tous nos patients.

Conclusion: Le poumon éosinophile est une affection grave, voire mortelle. Une enquête étiologique rigoureuse est nécessaire pour adapter la prise en charge thérapeutique qui reste basée sur la corticothérapie.

Conflits d'intérêt: Rien à déclarer 


\section{P112 \\ Etude Comparative de la Prise en Charge Thérapeutique de la Rhinite Allergique Associée ou Non à l'asthme}

\section{S. Aidou, H. Arfaoui, H. Jabri, W. Elkhattabi, H. Afif}

Service des Maladies Respiratoires, Hôpital 20 Août 1953, CHU Ibn Rochd

Introduction: Les liens entre asthme et rhinite allergique sont particulièrement étroits, la plupart des asthmatiques présentent aussi des rhinites allergiques (80\% des cas), et environ $45 \%$ des personnes touchées par une rhinite développeront aussi un asthme.

Méthode: Nous avons mené une étude rétrospective à la consultation d'allergologie de l'hôpital 20 Aout de Casablanca, portant sur 244 dossiers de patients porteurs de rhinite allergique répartis en deux groupes. Le groupe 1 comporte 142 patients ayant une rhinite allergique associée à un asthme, le groupe 2 comprend 102 patients ayant une rhinite allergique non associée à l'asthme, sur une période d'étude allant de Juillet 2015 à Juillet 2019.

Résultats: La moyenne d'âge est de 29 ans dans le groupe 1 vs 36 ans dans le groupe 2, avec une nette prédominance féminine $63 \%$ vs $71 \%$. L'humidité et le non-ensoleillement de l'habitat sont retrouvés dans $46 \%$ dans le groupe 1 vs $32 \%$ dans le groupe 2 . L'atopie est rapportée dans $49 \%$ dans le groupe 1 vs $47 \%$ dans le groupe 2. L'âge moyen du début était de 22 ans vs 28 ans. La rhinite allergique était persistante modérée à sévère dans $44 \%$ vs $34 \%$ des cas et intermittente dans $56 \%$ vs $60 \%$ des cas. Les TCA sont positifs chez la majorité des patients dans les deux groupes, principalement aux acariens DP $62 \%$ dans le groupe 1 vs $40 \%$ groupe 2 . Le traitement est à base d'antihistaminique $63 \%$ vs $64 \%$ et de corticoïde nasal $75 \%$ vs $76 \%$, avec une bonne évolution. Par ailleurs, une aggravation de la symptomatologie est notée chez $9 \%$ des patients du groupe 1 vs $5 \%$ du groupe 2 au cours de la période de pollinisation et ou à cause d'une mauvaise observance du traitement. La rhinite était la cause du non-contrôle de l'asthme dans 25\% des cas du groupe 1 .

Conclusion: Le diagnostic et le traitement de la rhinite allergique demeurent indispensables afin d'éviter les complications et d'améliorer le contrôle de l'asthme s'il est associé et ainsi la qualité de vie des patients.

Conflits d'intérêt: Rien à déclarer

\section{P113 \\ Manifestations Thoraciques des Lymphomes: Étude Comparative Entre LH et LMNH}

\section{S. Aidou, W. Elkhattabi, H. Arfaoui, H. Jabri, H. Afif}

Service des Maladies Respiratoires, Hôpital 20 Août 1953, CHU Ibn Rochd

Introduction: Les lymphomes malins non Hodgkiniens et Hodgkiniens ( $\mathrm{LMN}$ et $\mathrm{LH}$ ) traduisent une prolifération maligne des cellules lymphoïdes. Les atteintes thoraciques peuvent être primitives ou secondaires comprenant des localisations médiastinales, parenchymateuses, pleuro-péricardiques et pariétales. Notre étude vise à préciser les particularités cliniques et radioanatomiques des lymphomes thoraciques.

Méthode: Il s'agit d'une étude rétrospective incluant les cas d'atteintes thoraciques révélant un lymphome malin chez des patients hospitalisés au service de pneumologie de l'hôpital 20 Août entre janvier 2013 et juin 2020.

Résultats: Nous avons colligé 45 cas de lymphome thoracique dont $73 \%$ sont de type non hodgkinien. $71 \%$ des cas de LMNH sont de sexe masculin versus $66 \%$ des cas de LH. L'âge moyen dans le LMNH est de 33 ans versus 35,4 ans dans le LH. La symptomatologie clinique est dominée dans les 2 groupes par la toux, la dyspnée et la douleur thoracique. Le bilan radiologique objective une atteinte médiastinale dans $93 \%$ des cas de LMNH et $90 \%$ des cas de $\mathrm{LH}$, suivie par l'atteinte pleurale notée dans $49 \%$ des cas de LMNH. Les autres structures intrathoraciques sont moins fréquemment atteintes. Le diagnostic est histologique confirmé par biopsie pleurale dans 47,43\%, biopsie scannoguidée de la masse médiastinale dans $62,96 \%$ ou par biopsie ganglionnaire périphérique dans $38,46 \%$ pour le $\mathrm{LMNH}$, et par biopsie ganglionnaire périphérique dans $56,66 \%$ et une biopsie chirurgicale dans un cas pour LH.

Conclusion: À la lumière de cette étude, nous insistons sur la fréquence considérable et la variété des manifestations thoraciques des lymphomes qui sont dominées par l'atteinte médiastinale, suivie par celle pleurale.

Conflits d'intérêt: Rien à déclarer

\section{P114 \\ Les Manifestations Extrathoraciques Associées à la Sarcoïdose Thoracique}

\section{S. Aidou, H. Arfaoui, H. Jabri, W. Elkhattabi, H. Afif}

Service des Maladies Respiratoires, Hôpital 20 Août 1953, $\mathrm{CHU}$ Ibn Rochd

Introduction: La sarcoïdose est une granulomatose systémique d'étiologie inconnue dont la principale localisation est médiastinopulmonaire. Le granulome sarcoïdien peut être présent dans la quasi-totalité des organes provoquant ainsi des tableaux cliniques extrêmement polymorphes et de gravite variables menaçant parfois le pronostic fonctionnel et/ou vital.

Méthode: Il s'agit d'une étude rétrospective portant sur 105 cas de sarcoïdose thoracique colligés au service de pneumologie 20 Août 1953 de Casablanca entre janvier 2011 et juillet 2020.

Résultats: La moyenne d'âge était de 46 ans avec une prédominance féminine (82\%). La sarcoïdose était de type 0 dans $1 \%$ des cas, de type I dans $41 \%$ des cas, de type II dans $46 \%$ des cas et de type III dans $11 \%$ des cas. Des manifestations extra thoraciques étaient retrouvées chez 50 patients soit $47.6 \%$ des cas. Il s'agissait essentiellement de localisation nasale dans 12 cas, cutanée dans 9 cas et oculaire dans 7 cas. La localisation salivaire et ganglionnaire périphérique était retrouvée dans 7 cas chacune. La localisation 
neurologique et rénale dans 2 cas chacune et une localisation musculaire, cardiaque, splénique et hépatique dans un cas chacun. Le traitement était à base de corticothérapie orale dans 27 cas devant des atteintes respiratoires sévères et/ou des atteintes extra thoraciques mettant en jeu le pronostic vital ou fonctionnel. L'abstention thérapeutique était le choix dans les 60 cas. Un traitement à base d'hydroxy chloroquine et de corticothérapie locale était retrouvé dans 10 cas pour des atteintes cutanées (visage).

La sarcoïdose est une maladie systémique qui peut affecter tous les organes du corps posant souvent des problèmes diagnostiques, pouvant menaçant le pronostic fonctionnel et/ou vital. Le traitement peut varier selon l'organe atteint mais les corticoïdes restent le traitement de choix quand ce dernier est indiqué.

Conflits d'intérêt: Rien à déclarer

\section{P115 \\ Les Causes du Retard Diagnostique dans le Cancer Bronchogénique}

S. Aidou, W. Elkhattabi, H. Arfaoui, H. Jabri, H. Afif

Service des Maladies Respiratoires, Hôpital 20 Août 1953, CHU Ibn Rochd

Introduction: Le cancer bronchogénique constitue un problème majeur de santé publique. Son diagnostic est le plus souvent tardif. Le but de notre étude est de relever les différentes causes du retard diagnostique et thérapeutique du cancer bronchogénique.

Méthode: Nous avons mené une étude rétrospective descriptive et analytique, portant sur 170 patients hospitalisés entre Avril 2014 et Janvier 2019.

Résultats: L'âge moyen de nos patients était de 60 ans avec prédominance masculine à $91 \%$, le tabagisme a été retrouvé dans $86 \%$ des cas. Les signes cliniques étaient dominés par la douleur thoracique ( $80 \%$ des cas). Le carcinome bronchogénique non à petites cellules était le type histologique le plus fréquent dont $58 \%$ étaient des adénocarcinomes. Le stade IV a été retrouvé dans 59\% au moment du diagnostic. Le délai médian de consultation était de 49 jours, le délai médian du diagnostic était de 22 jours et le délai médian thérapeutique était de 13 jours. Le délai global médian de prise en charge était de 130 jours. Les facteurs associés à un retard diagnostique étaient le sexe féminin, l'absence du tabagisme et la présence des signes extra-respiratoires.

Conclusion: Notre travail est une invitation à produire d'autres études permettant de comparer les délais dans plusieurs régions du Maroc en fonction de divers paramètres. Ceci permettra d'obtenir une large base de données nationale, et de fonder des recommandations nationales adaptées à notre contexte.

Conflits d'intérêt: Rien à déclarer

\section{P116 \\ Insomnie et Pandémie COVID-19 Chez le Cadre Soignant en Service de Pneumologie}

\section{S. Hajjej, S. Aissa, S. Bouafia, W. Benzarti, A. Knaz, A. Abdelghani, A. Garrouche, A. Hayouni, M. Benzarti, I. Gargouri}

Service de Pneumologie CHU Farhat Hached de Sousse, Tunisie

Introduction - Objectifs: Plusieurs études dans le monde, notamment chinoises, ont rapporté divers aspects de retentissement psychologiques de la pandémie COVID-19, entre autres l'insomnie qui touchaient environ $35 \%$ du cadre soignant. L'objectif de ce travail est d'évaluer la prévalence de l'insomnie chez le cadre soignant en service de pneumologie.

Matériels et méthodes: Une étude rétrospective a été menée au service de Pneumologie du CHU Farhat Hached à Sousse, intéressant le cadre soignant: ils ont tous répondu au questionnaire Index de sévérité de l'insomnie (ISI) au cours de la lére vague du covid-19 (la période entre mars 2020 et mai 2020). Ce questionnaire comporte 7 questions cotées de 0 à 4 . Si l'index est supérieur à 22, la symptomatologie est sévère, entre 15 et 21 , modérée, entre 8 et 14 , légère, et s'il est inférieur à 7 , absence de symptomatologie. A noter que durant cette période, le service à hospitalisé, en unité d'isolement, 59 patients adressés par le biais des urgences pour suspicion de COVID-19 et que tout le cadre soignant a eu une formation concernant les précautions à prendre face à cette pandémie.

Résultats: Parmi le cadre soignant, 38 ont rempli le questionnaire (50\% cadre médical et $50 \%$ cadre paramédical), avec un taux de participation à $71 \%$. L'âge moyen était de 35,05 ans $\pm 10,57$ avec des extrêmes entre 24 ans et 65 ans. Le sex-ratio était à 0,35 (10 hommes contre 28 femmes). En moyenne, le nombre des années d'expérience professionnelle était à 10 ans $\pm 9,84$ avec des extrémités allant de 1 an à 35 ans. Un travail posté a été noté dans 39\% des cas. $52,6 \%$ étaient mariés et $68,4 \%$ vivaient en famille. $92,1 \%$ ont un niveau d'étude universitaire. Seulement 4 participants étaient tabagiques (tous des hommes), 18,4\% avaient des maladies chroniques et $7,9 \%$ avaient des antécédents psychiatriques auparavant (dépression). 57,9\% avaient en leur compagnie soit un sujet âgé de plus de 65 ans, soit un sujet avec des maladies chroniques ou une immunodépression. 15 sujets ont augmenté leur consommation du café, et aucun sujet ne prenait des substances psychoaddictives. Des troubles du sommeil ont été observés dans $42,1 \%$ des cas, et ils ont été dominés par: diminution des heures du sommeil (13 cas), des cauchemars ( 4 cas) et des difficultés d'endormissement (4 cas). L'insomnie était sévère dans 2 cas, modérée dans 5 cas et légère dans 6 cas. Elle a été corrélée au sexe féminin $(p=0,05)$, ceux qui étaient entourés par un sujet âgé de plus de 65 ans, soit un sujet avec des maladies chroniques ou une immunodépression $(\mathrm{p}=0,052)$, ceux ayant des antécédents psychiatriques $(\mathrm{p}=0.01)$, et ceux qui ont présenté une symptomatologie de dépression $(\mathrm{p}=0.005)$. Paradoxalement, elle n’a pas été corrélée au travail posté. La prise des somnifères a été notée chez 5 personnels.

Conclusion: En période de pandémie, une attention particulière doit être portée sur les troubles du sommeil chez le cadre soignant, notamment l'insomnie dont la prévalence est estimée dans notre étude à $34 \%$. Leur prise en charge permettra certainement d'améliorer la qualité des soins dans nos hôpitaux.

Conflits d'intérêt: Rien à déclarer
SSP/SSTS - EFP

Joint Annual Conference 2021 


\section{P117 \\ Impact de la Pandémie COVID-19 sur la Qualité de vie du Cadre Soignant en Service de Pneumologie}

S. Hajjej, S. Aissa, S. Bouafia, W. Benzarti, A. Knaz, A. Abdelghani, A. Garrouche, A. Hayouni, M. Benzarti, I. Gargouri

Service de Pneumologie CHU FarhatHached de Sousse, Tunisie

Introduction - Objectifs: La pandémie COVID-19 a provoqué une crise sanitaire majeure avec un bouleversement de la vie quotidienne familiale, sociale et professionnelle de la population générale et du cadre soignant, en particulier ceux qui sont en première ligne. L'objectif de ce travail est d'évaluer l'impact de cette pandémie sur la qualité de vie du cadre soignant en service de pneumologie.

Matériels et méthodes: Une étude rétrospective a été menée au service de Pneumologie du CHU Farhat Hached à Sousse, intéressant le cadre soignant: ils ont tous répondu au questionnaire Sf-36 qui évalue la qualité de vie au cours de la lére vague du covid-19 (la période entre mars 2020 et mai 2020). Ce questionnaire comporte 1litems qui sont par la suite regroupés en 2 scores synthétiques: santé physique et santé mentale. A noter que durant cette période, le service à hospitalisé, en unité d'isolement, 59 patients adressés par le biais des urgences pour suspicion de COVID-19 et que tout le cadre soignant a eu une formation concernant les précautions à prendre face à cette pandémie.

Résultats: Parmi le cadre soignant, 38 ont rempli le questionnaire (50\% cadre médical et $50 \%$ cadre paramédical), avec un taux de participation à $71 \%$. L'âge moyen était de 35,05 ans $\pm 10,57$ avec des extrêmes entre 24 ans et 65 ans. Le sex-ratio était à 0,35 (10 hommes contre 28 femmes). En moyenne, le nombre des années d'expérience professionnelle était à 10 ans $\pm 9,84$ avec des extrémités allant de 1 an à 35 ans. Un travail posté a été noté dans $39 \%$ des cas. $52,6 \%$ étaient mariés et $68,4 \%$ vivaient en famille. $92,1 \%$ ont un niveau d'étude universitaire. Seulement 4 participants étaient tabagiques (tous des hommes), 18,4\% avaient des maladies chroniques et $7,9 \%$ avaient des antécédents psychiatriques auparavant (dépression). 57,9\% avaient en leur compagnie soit un sujet âgé de plus de 65 ans, soit un sujet avec des maladies chroniques ou une immunodépression. 7 personnes avaient une santé physique moyenne et le reste en bonne santé. Une corrélation statistiquement significative a été trouvé entre la santé physique et ceux ayant des antécédents psychiatriques $(\mathrm{p}=0.001)$, ceux présentant une dépression ( $\mathrm{p}=0,05)$ ou une insomnie sévère $(\mathrm{p}=0,009) .9$ personnes avaient une santé mentale moyenne contre 7 ayant une mauvaise santé mentale. La santé morale a été corrélée à l'anxiété et à la dépression $(\mathrm{p}=0,001)$.

Conclusion: Cette crise sanitaire devrait nous aider à mieux comprendre la vulnérabilité des soignants à la souffrance physique et psychologique Améliorer leur qualité de vie surtout en période de pandémie devient un enjeu important pour garantir une meilleure prise en charge des patients.

Conflits d'intérêt: Rien à déclarer

\section{P118 \\ L'infection à Pseudomonas Aeruginosa au Cours des Bronchiectasies: Caractéristiques Cliniques et Évolutives}

\section{S. Toujani, A. Hedhli, M. Kacem, Y. Ouahchi, A. Naaroura, S. Cheikhrouhou, S. Merai, M. Mjid, B. Dhahri}

Service de Pneumologie CHU La Rabta, Tunis, Tunisie

Introduction: La survenue d'une surinfection à pyocyanique au cours des bronchiectasies est considérée comme un tournant évolutif de la maladie. L'objectif de cette étude est d'évaluer le profil des patients atteints de bronchiectasies hors mucoviscidose présentant des infections bronchiques à pyocyanique ainsi que leurs conséquences sur l'évolution de la maladie.

Matériel et méthodes: Il s'agit d'une étude rétrospective sur dossiers de patients présentant des bronchiectasies diffuses et bilatérales hors mucoviscidose, hospitalisés au Service de Pneumologie la Rabta, Tunis pour exacerbation (EA). Deux groupes sont comparés: G1: patients avec surinfection à pyocyanique; G2: patients sans surinfection à pyocyanique.

Résultats: L'étude a intéressé 100 patients: 58 hommes et 42 femmes âgés en moyenne de 60,1 $\pm 14,57$ ans. La surinfection bronchique à Pyocyanique était notée chez 29 patients (G1) avec un portage chronique dans 20 cas. Les 2 groupes sont comparables en termes de comorbidités. Le G1 est plus symptomatique que le G2 (dyspnée: $85,4 \%$ vs $60 \% ; \mathrm{p}=0,048$ ). Le nombre d'exacerbation/an était plus important dans $\mathrm{G} 1(3,9$ vs $2,1 \%$; $\mathrm{p}=0,045)$ avec une CVF plus basse $(1,5 \mathrm{~L}$ vs $1,761 \mathrm{~L} ; \mathrm{p}=0,06)$ et un VEMS plus bas $(0,950 \mathrm{~L}$ vs 1,$220 ; p=0,038)$. Les EA du G1 sont plus sévères caractérisées par une $\mathrm{PaO} 2$ plus basse $(60 \mathrm{mmHg}$ vs $75 \mathrm{mmHg}$; $\mathrm{p}=0,055)$, avec plus de recours aux soins intensifs $(21,4 \%$ vs $9 \% ; \mathrm{p}=0,048)$, et un syndrome inflammatoire plus fréquent $(70 \%$ vs $57,1 \%$; $p=0,05)$. La durée d'hospitalisation était plus longue. L'évolution à long terme est marquée par l'installation d'une insuffisance respiratoire chronique sévère dans $(42,8 \%$ vs $25,7 \%$; $\mathrm{p}=0,04)$.

Conclusion: La surinfection bronchique à pyocyanique est une complication classique au cours des bronchiectasies. Le diagnostic bactériologique et la mise en route précoce du traitement sont essentiels afin d'éviter la colonisation chronique et la détérioration de la fonction respiratoire chez cette population.

Conflits d'intérêt: Rien à déclarer 


\section{P119 \\ Impact des Comorbidités Cardiaques Sur le \\ Pronostic de l'exacerbation Aigue de BPCO}

S. Toujani, Boubaker N, A. Hedhli, Y. Ouahchi, S. Cheikh Rouhou, S. Merai, M. Mjid, B. Dhahri.

Service de Pneumologie la Rabta Tunis Tunisie

Introduction: Les patients atteints de broncopneumopathie chronique obstructive (BPCO) ont souvent d'autres pathologies chroniques. Ces comorbidités ont un impact important sur les symptômes, le pronostic vital et les coûts de traitement de la maladie.

Objectif: Déterminer l'impact des comorbidités cardiovasculaires sur la sévérité et le pronostic des patients admis pour exacerbation aigue (EA) de BPCO.

Méthodes: Il s'agissait d'une étude rétrospective portant sur les dossiers de 200 patients admis dans notre service pour EA de BPCO entre janvier 2014 et juillet 2019. Les patients ont été divisés en deux groupes: le G1 $(n=104)$ incluait les patients ayant une comorbidité cardiovasculaire ou plus (cardiopathie ischémique, arythmie, insuffisance cardiaque, HTA, AVC) et le G2 ( $n=96)$ représentait les patients sans comorbidités cardiovasculaires.

Résultats: Nous avons colligé $93 \%$ patients de sexe masculin avec un âge moyen de 63,45 $\pm 10,9$ ans. Avant l'admission, la maladie était classée GOLD A, B, C ou D respectivement dans 7\%, $34 \%$, $28 \%$ et $31 \%$ des cas. L'hypertension artérielle était la comorbidité cardiovasculaire la plus fréquente (60\% des cas). Les patients du G1 étaient plus symptomatiques. En effet, une dyspnée stade $m M R C \geq 2$ était plus retrouvée dans le G1 (79\% versus 65,6\%; $\mathrm{p}=0,06)$. L'examen à l'admission montrait des signes de lutte chez $67,6 \%$ du G1 contre 59,4\% de G2; ( $p=0,2)$, d'insuffisance cardiaque droite chez 30,5\% du G1 contre 11,5\% du G2; $(p=0,01)$ et des signes d'encéphalopathie hypercapnique dans $15,2 \%$ des cas du G1 contre $10,4 \%$ du G2; $(\mathrm{p}=0,06)$. La gazométrie artérielle à l'admission montrait une $\mathrm{PaO} 2$ moyenne plus basse $(60 \mathrm{mmHg}$ versus $67 \mathrm{mmHg}$; $=0,05)$ et une $\mathrm{PaCO} 2$ moyenne plus élevée dans le G1 (59 $\mathrm{mmHg}$ versus $53 \mathrm{mmHg} ; \mathrm{p}=0,06)$. Le recours à la ventilation non invasive (VNI) était plus fréquent dans le G1 (40,4\% versus $37 \% ; \mathrm{p}=0,06)$. Malgré le traitement médical, une aggravation de l'état respiratoire nécessitant le transfert en réanimation était plus fréquent dans le $\mathrm{G} 1(\mathrm{p}=0,04)$ avec plus de recours à la ventilation mécanique $(16,2 \%$ versus $9,5 \%$; $=0,03)$. A la sortie, $31,6 \%$ des patients du G1 étaient mis sous une oxygénothérapie à domicile $(p=0,03)$ et $22,4 \%$ sous une VNI au long cours $(p=0,02)$.

Conclusion: Les comorbidités cardiovasculaires semblent avoir un impact péjoratif sur la présentation clinique et l'évolution des EA de BPCO. En conséquence, une prise en charge adéquate et précoce de ces pathologies est primordiale.

Conflits d'intérêt: Rien à déclarer

\section{P120 \\ Rôle du Tabac dans le Non Contrôle de L'asthme}

S. Raftani, N. Bougteb, H. Arfaoui, H. Jabri, W. Elkhattabi, M. H. Afif

Service des Maladies Respiratoires, Hôpital 20 Août 1953, CHU Ibn Rochd

Introduction: Le Tabac est parmi les causes majeures d'exacerbation et de perte de contrôle de l'asthme. Les asthmatiques fumeurs ou anciens fumeurs représentent $40 \%$ des patients asthmatiques. Cette population à une qualité de vie moins bonne par rapport au reste des patients asthmatiques.

Méthode: Nous avons mené une étude rétrospective au centre de consultation d'allergologie de l'hôpital 20 Aout 1953 de Casablanca sur une période de janvier 2014 à janvier 2020.

Résultats: 160 dossiers de patients asthmatiques ont été colligés, répartis en 2 groupes, le groupe 1 comprenait 60 fumeurs, le groupe 2 comprenait 100 non-fumeurs. Les asthmatiques fumeurs représentaient $37.5 \%$ de l'ensemble de nos patients. L'âge moyen était de 42 ans dans le groupe 1 versus 29 ans dans le groupe 2 , dominés par le sexe masculin $91 \%$. Les exacerbations sévères étaient plus fréquentes dans le groupe 1. Le contrôle était mauvais dans $92 \%$ dans le groupe $1,8 \%$ des patients du groupe 1 étaient contrôlés versus $40 \%$ du groupe 2 , aucun patient du groupe 1 n'avait un contrôle total sur son asthme contrairement au groupe 2 avec un contrôle total de $26 \%$ chez ces patients non fumeurs. Le VEMS/CVF était en moyenne de $51 \%$ dans le groupe 1 et de $66 \%$ dans le groupe 2 . Les patients du groupe 1 étaient tous sous corticothérapie inhalée associée aux bronchodilatateurs de longue durée d'action. $60 \%$ des patients du groupe 2 étaient sous corticothérapie inhalée associée aux bronchodilatateurs de longue durée d'action et $15 \%$ sous corticothérapie inhalée seule.

Conclusion: Le contrôle de l'asthme chez le fumeur semble plus difficile que celui du non-fumeur, le tabac entretient l'inflammation bronchique et diminue l'efficacité de la corticothérapie, d'où l'importance du sevrage tabagique chez les asthmatiques.

Conflits d'intérêt: Rien à déclarer

\section{P121 \\ Profil Clinique et Étiologique des Goitres Plongeants (A Propos de 15 Cas)}

S. Raftani, W. Elkhattabi, H. Bamha, H. Arfaoui, H. Jabri, M. H. Afif

Service des Maladies Respiratoires, Hôpital 20 Août 1953, CHU Ibn Rochd, Casablanca

Introduction: Les goitres plongeants sont des tumeurs thyroïdiennes bénignes ou malignes retrouvées en totalité ou en partie dans le thorax. Il s'agit dans la majorité des cas d'un goitre bénin. Sur le plan clinique, les goitres plongeants sont latents dans $20 \%$ des cas. Les signes cliniques se divisent en signe de dysthyroïdie et en signes de compression. 
Méthode: Notre travail est une étude analytique de 15 cas, colligés dans notre service entre janvier 1991 et juillet 2020 .

Résultats: Nous avons noté une légère prédominance féminine avec une moyenne d'âge de 61 ans. La symptomatologie clinique est faite surtout de signes compressifs, dominés par la dysphonie rencontrée dans 9 cas. Les signes de dysthyroïdie sont retrouvés dans deux cas. Le goitre a été palpé chez 10 malades. La radiographie thoracique a objectivé un élargissement médiastinal supérieur dans 15 cas associé à une déviation de la trachée dans 8 cas. Le scanner thoracique réalisé chez 8 patients a objectivé un goitre plongeant dans tous les cas. Tous nos patients ont bénéficié d'une cervicotomie de Kocher. L'étude histologique avait conclu à la bénignité dans tous les cas. L'évolution était bonne chez tous nos patients.

Conclusion: Même si les goitres plongeants sont souvent bénins, l'étude histologique est indispensable, le traitement étant chirurgical, la cervicotomie est généralement suffisante.

Conflits d'intérêt: Rien à déclarer

\section{P122 \\ Apport de la Ponction Biopsie Pleurale dans le Diagnostic des Pleurésies Malignes}

\section{S. Raftani, W. Elkhattabi, S. Msika, H. Arfaoui, H. Jabri, M.H. Afif}

Service des Maladies Respiratoires, Hôpital 20 Août 1953,

CHU Ibn Rochd, Casablanca

Introduction: Dans notre contexte, deux grandes étiologies prédominent au cours des pleurésies. La tuberculose pleurale et la pleurésie d'origine maligne. Le but de notre travail est de déterminer l'apport de la ponction biopsie pleurale dans les pleurésies tumorales.

Méthode: Il s'agit d'une étude rétrospective portant sur les dossiers de patients hospitalisés pour pleurésie d'origine maligne très probable devant la présence d'au moins 2 de ces critères: douleur thoracique, pleurésie abondante et ou récidivante, pleurésie hémorragique.

Résultats: Les pleurésies tumorales sont retrouvées dans 170 cas (42.5\%). Le sexe masculin est dominant dans 120 cas (70\%), avec une moyenne d'âge de 55 ans. Le tabagisme est retrouvé dans 78 cas (45\%). Le syndrome d'épanchement liquidien est retrouvé dans 162 cas (95\%). La radiographie thoracique complétée par la tomodensitométrie a montré une opacité de type pleural dans tous les cas. La ponction biopsie pleurale était contributive dans 128 cas (75\%). Elle était contributive dès le premier geste dans 90 cas (70\%) et après deux à plusieurs gestes dans 38 cas (30\%). La nature histologique était dominée par le carcinome épidermoïde dans 75 cas $(44 \%)$.

Conclusion: L'apport de la ponction biopsie pleurale est non négligeable et constitue l'examen de première intention. La multi- plication des gestes est importante lorsque les premières ponctions biopsies pleurales ne sont pas contributives, afin de ne pas traiter à tort les patients pour tuberculose pleurale même si, nous sommes dans une zone endémique.

Conflits d'intérêt: Rien à déclarer

\section{P123 \\ Caractéristiques Cliniques, Paracliniques et Résultat du Traitement des Patients Atteints de L'infection Fongique Pulmonaire Invasive}

VU. ThiNhinh, VU. VanGiap

Service de médecine interne 4, Hôpital Viet Tiep, Hai Phong, Vietnam

Objectif: Décrire les caractéristiques cliniques, paracliniques et évaluer lerésultat du traitement des patients atteints de l'infection fongique pulmonaire invasive.

Population et méthode: Étude descriptive prospective sur 22 patients atteints de l'infection fongique pulmonaire invasive traitée au Centre respiratoire de l'hôpital BachMai au VietNam de janvier 2020 à décembre 2020.

Résultats: Environ 2/3 patients atteints de l'infection fongique invasive étaient masculins, le groupe de patient de plus de 60 ans était à $63.6 \%$, la BPCO/Asthme (27,3\%), la diabète de type 2 (28.6\%) étaient les cormobidités plus fréquentes. $45,5 \%$ parmi des patients ayant le facteur atopique, et la prise de corticostéroïdes prolongée était la plus fréquente $(50 \%)$. Le motif d'entrée très multiforme: fièvre $(27,3 \%)$, dyspnée $(22,7 \%)$, toux productive (22.7\%), hémoptysie $(18,3 \%)$, toux sèche $(4,5 \%)$, fatigue $(4,5 \%)$. $22,7 \%$ patients avaient aucune signe générale et signe respiratoire. Sur le scanner, plus de la moitié des patients avaient la lésion sur touts les deux poumons avec la condensation prédominante $(54,5 \%)$. La forme lésion de bronchite purulente sur la fibroscopie bronchique était très très élévée $(71,4 \%)$. L'Amphotericin $B$ deoxylate était la choix de médicament antifongique fréquente pour le traitement initial $(36,4 \%)$, les motifs alternatifs courants étaient la toxicité rénale $(21,4 \%)$, l'antibiogramme fongique résistant au médicament initial $(35,7 \%)$, la toxicité hépatique $(7,1 \%)$, les autre motifs $(35,8 \%)$. Le taux de combinaison antibiotique était $68,2 \% .13$ patients $(50,1 \%)$ avaient l'effet secondaire (hypokalémie $46,2 \%$, insuffisance rénale $23 \%$, augmentation de transaminase $30,8 \%)$. Le résultat clinique à la sortie: diminution $(63,6 \%)$, pas de changement $(18,2 \%)$ aggravation $(9,1 \%)$, décès $(9,1 \%)$.

Conclusion: Cette étude présentait les signes cliniques et paracliniques de infection fongique invasive multiforme, non spécifique mais le dignostic et le traitement précoce pouvait améliorer le résultat à la sortie.

Conflits d'intérêt: Rien à déclarer 


\section{P124 \\ La Tuberculose Multifocale au Centre hospitalier et Universitaire (CHU) de Libreville, Libreville, Gabon: Influence de l'infection à VIH}

Ulrich Davy Kombila, Charleine Manomba Boulingui,

Dieudonné Mounguengui, Linda Daniel Ibinda, Josaphat Iba Ba, Jean Bruno Boguikouma

Service de Médecine Interne, CHU de Libreville

Introduction: L'objectif de l'étude était de décrire les aspects cliniques et évolutifs de la tuberculose (TB) multifocale et d'apprécier l'impact de l'infection à VIH.

Méthode: Etude rétrospective, descriptive et analytique, à partir des dossiers des malades suivis pour TB multifocale au service de médecine interne du CHU de Libreville de Janvier 2018 à Décembre 2019.

Résultats: Des 73 patients inclus, $22(30,1 \%)$ avaient une infection à VIH. Ces résultats laissent apparaitre une prédominance féminine (58,9\%) avec un âge moyen de 33,5 111,4 ans. La majorité des patients avait moins de 45 ans (89\%). Les formes avec atteinte bifocale extrapulmonaire et trifocale étaient respectivement de $65,8 \%$ et $34,2 \%$. L'atteinte pulmonaire était associée dans $34,2 \%$. Le délai moyen de consultation: 123,2 jours [17-730]. Le diagnostic de TB était confirmé histologiquement et/ou bactériologiquement dans les mêmes proportions (35,6\%). Les facteurs de comorbidités retrouvés étaient le lupus érythémateux aigu disséminé (2,7\%), la drépanocytose $(1,4 \%)$, l'insuffisance rénales chronique $(1,4 \%)$, l'hypertension artérielle $(1,4 \%)$. Le taux de succès thérapeutique était de $74,5 \%$ chez les patients VIH négatifs $(\mathrm{p}=0,46)$, alors que le taux d'abandon de traitement était de $64,7 \%$ chez les patients VIH positifs ( $p=0,678$ ). Les patients VIH positifs étaient moins susceptibles d'avoir une biopsie positive $(\mathrm{p}=0,021)$. Les patients atteints de TB multifocale positif au VIH étaient moins susceptibles à présenter une TB multifocale avec deux atteintes extrapulmonaires $(\mathrm{p}=0,002)$.

Conclusion: la TB multifocale n'est pas uniquement l'apanage des patients VIH positif. Elle peut également survenir sur les terrains immunocompétents au VIH. Savoir rechercher des formes pour une meilleure prise en charge, partant un meilleur pronostic.

Conflits d'intérêt: Rien à déclarer

\section{P125 \\ Profil Épidémiologique et Évolutif de la Tuberculose Sous l'influence de l'infection par le VIH/SIDA Dans un Centre de Prise en Charge Ambulatoire de la Tuberculose au Gabon}

\author{
Ulrich Davy Kombila, Charlène Manomba Boulingui, \\ Linda Danielle Ibinga, Dieudonné Mounguengui, \\ Jean Bruno Boguikouma ${ }^{1}$
}

Service de Médecine Interne, CHU de Libreville

Introduction: Très peu de travaux abordent l'épidémiologie de la tuberculose à Libreville. Pour comprendre l'impact de l'infection à VIH sur l'épidémiologie de la TB dans un centre de prise en charge ambulatoire, nous avons réalisé cette étude.

Patients et méthode: Le registre de déclaration des cas de TB du CDT du CHU de Libreville d'octobre 2017 à septembre 2019 a été exploité. Les analyses en régression logistique bivariée et multivariée ont été effectuées pour évaluer l'impact de l'infection à VIH sur la TB.

Résultats: La prévalence de l'infection à VIH était de 30,4\%. La proportion des femmes était de 51,2\% avec une moyenne d'âge de 35,2 ans et des extrêmes de 15 et 83 ans. La prévalence du tabagisme était de 33,9\%. Le délai diagnostic était de 104,7 jours $(\mathrm{ET} \pm 81,2)$ soit 3,5 mois $(\mathrm{ET} \pm 2,7)$ environ. Les nouveaux cas avaient constitué $92,6 \%$ des cas de TB. Dans 47,8\%, il s'agissait d'une TPM+. Au terme du traitement le devenir des patients était: $40,8 \%$ guérison, $25,9 \%$ traitement complété, $27,3 \%$ perdu de vue, $1,5 \%$ transfert, $4,6 \%$ décès. Le succès thérapeutique avait constitué $66,7 \%$ de l'issu du traitement. En analyse multivariée, les sujets de sexe féminin ont été plus infectés par le VIH ( $\mathrm{p}=0,002 ; \mathrm{OR}=1,960 \mathrm{IC} 95 \%[1,275$ - 3,015]). L'infection à VIH a été associée à une réduction de la proportion de TPM (+) $(\mathrm{p}=0,001 ; \mathrm{OR}=0,483$ IC95\% [0,311$0,752]$ ), à une augmentation de la proportion de nouveau cas ( $p=0,007 ; O R=2,987$ IC95\% [1,353-6,597]), et de tuberculose extrapulmonaire $(p<0,001 ; O R=1,084$ IC95\% [1,054-1,131], enfin à une évolution thérapeutique défavorable $(\mathrm{p}=0,016 ; \mathrm{OR}=2,744$ IC95\% [1,208-6,233]).

Conclusion: La co-infection TB/VIH reste élevé. Elle impacte négativement sur la prise en charge de la TB en ambulatoire.

Conflits d'intérêt: Rien à déclarer
P126

Comparison of Forced and Slow Vital Capacity Maneuvers in Defining Airway Obstruction in Asthmatic Patients with Normal Spirometry

Ferdaous Yangui, Soumaya Debbiche, Héla Cherif, Mariem Triki, Bochra Karaborni, Maroua Bougacha, Mohamed Ridha Charfi

Pneumology department. FSI Hospital. La Marsa. Tunis

Introduction: Obstructive lung disease is diagnosed by a decreased ratio of FEV1 to the vital capacity (VC). Although the 
most used VC is FVC, slow VC (SVC), may offer a more accurate evaluation of breathing capacity. There is recent evidence that using only FEV1/FVC underrecognizes obstruction in asthmatic patients without obstructive ventilatory disorder. Thus, using only FEV1/SVC may be helpful in diagnosing obstruction in symptomatic asthmatics.

We aimed to evaluate the difference between FVC and SVC in defining airway obstruction in asthmatic patients.

Methods: A cross-sectional study was conducted in FSI hospital in Tunisia for two months and including asthmatic patients. All patients have normal spirometric parameters regarding to the ratio between FEV1 and FVC.

Results: A total of 30 asthmatic patients with a mean age of 57.2 \pm 7 years were included. The mean FEV1/FVC ratio was $81,33 \%$. However, when we use the ratio FEV1/SVC, the mean ratio was $75,87 \%$. By using FEV1/SVC, the prevalence of obstruction increased to $3 \%$.

Conclusion: The use of SVC maneuvers appears to decrease the prevalence of symptomatic patients with normal spirometry results and identify individuals with symptomatic obstructive lung disease. Recognizing the difference between SVC and FVC measurements in subjects will improve the testing and diagnosis of obstructive lung disease.

Disclosure: Nothing to disclose

\section{P127 \\ Étude des Volumes Pulmonaires Chez les Obèses Apnéiques}

Khadija Ayed, Imen Sahnoun, Salma Mokaddem, Hana Trabelsi, Saloua Ben Khamsa Jameleddine, Leila Douik El Gharbi

Service de Pneumologie D et service des Explorations

Fonctionnelles de l'Hôpital Abderrahmene Mami, Ariana,

Tunisie

Introduction: Le syndrome d'apnées hypopnées obstructives du sommeil (SAHOS) est une entité clinique caractérisée par la présence événements respiratoires anormaux pendant le sommeil. Il s'agit d'une pathologie fréquente dont l'obésité est un facteur de risque majeur et est corrélée à sa sévérité. Sur le plan respiratoire, l'obésité entraîne une atteinte restrictive, des modifications de la mécanique ventilatoire et une altération de la commande respiratoire.

Objectif: L'objectif de cette étude était d'évaluer l'impact du SAHOS sur les volumes pulmonaires chez les obèses apnéiques.

Méthode: Il s'agissait d'une étude transversale incluant 100 patients obèses (IMC $\geq 30 \mathrm{Kg} / \mathrm{m} 2$ ) n'ayant aucune pathologie respiratoire associée. Cette population a été subdivisée en deux groupes: le groupe $1(n=52)$ incluant les patients obèses apnéiques chez lesquels le diagnostic de SAHOS a été confirmé par une polygraphie ventilatoire, et le groupe $2(n=48)$ incluant les patients obèses sans SAHOS. L'étude des volumes pulmonaires a été réalisée à l'aide d'une pléthysmographie corporelle totale.

Résultats: Chez le groupe 1, l'index apnées-hypopnées (IAH) moyen était de 36,6 \pm 19,2/h et le SAHOS était sévère chez 71,2\% des cas. L'âge moyen était comparable chez les deux groupes (55 vs 58 ans; $\mathrm{p}=0,07)$. L'IMC moyen était significativement plus élevé dans le groupe $1(40,8$ vs $37 \mathrm{Kg} / \mathrm{m} 2 ; \mathrm{p}=0,002)$ et était fortement corrélée avec l'IAH $(\mathrm{p}=0,004)$. L'étude pléthysmographique des volumes pulmonaires a révélé une fréquence plus élevée du déficit ventilatoire restrictif (DVR) chez les patients du groupe 1 (23 vs $14,5 \%)$, cette différence était statistiquement non significative. En effet aucune corrélation n'a été trouvée entre l'IAH et le DVR. Cependant, cette anomalie fonctionnelle respiratoire était fortement corrélée avec l'IMC et avec la sévérité de l'obésité ( $\mathrm{p}=0,0001)$. Cette étude a révélé aussi une distension pulmonaire statique significativement plus fréquente et une capacité pulmonaire totale plus élevée chez les patients du groupe 1 ( 17 vs $8,3 \%$; $\mathrm{p}=0,01$ ), (5,45L vs $5,2 \mathrm{~L} ; \mathrm{p}=0,013)$.

Conclusion: Ainsi, selon notre étude, l'impact du SAHOS sur les volumes pulmonaires chez les obèses apnéiques réside surtout dans l'apparition d'une distension pulmonaires statiques et non pas dans l'apparition d'un DVR. Autres études sur des populations plus larges sont nécessaires pour mieux préciser le rôle du SAHOS dans le développement de cette distension pulmonaire statique.

Conflits d'intérêt: Rien à déclarer

\section{P128 \\ La Détection des Micrométastases du Cancer du Poumon dans les Ganglions Lymphatiques Par Cytométrie en Flux}

\section{A. Raniszewska, I. Kwiecien, E. Rutkowska, R. Sokolowski, J. Domagala-Kulawik}

Laboratoire d'hématologie et cytométrie en flux, Département de médecine interne et d'hématologie, Institut Militaire de Médecine

Introduction: On estime qu'environ vingt pour cent des patients atteints d'un cancer du poumon non à petites cellules (CPNPC) négatifs aux ganglions lymphatiques (LN) qui subissent une chirurgie d'intention curative ont des micro-métastases occultes détectables dans les $\mathrm{LN}$ réséqués. Au cours du paradigme de stadification standard du CPNPC, l'immunohistochimie reste le «gold standard» pour évaluer l'implication des ganglions lymphatiques. EBUS/TBNA est prometteur pour un examen précis des LN chez les patients atteints de CPNPC. Le but de l'étude était d'évaluer la présence de cellules cancéreuses du poumon dans les aspirats de LN en utilisant la cytométrie en flux (FC) et de la comparer avec les résultats d'échantillons de cytopathologie standard obtenus simultanément par EBUS/TBNA.

Méthode: Les analyses FC ont été effectuées sur des EBUS/ TBNA spécimens de 40 patients avec un CPNPC primaire confirmé. Les cellules cancéreuses du poumon ont été définies comme CD45-/EpCAM+/CXCR4+. Les LN ont été considérés comme métastatiques lorsque les spécimens de cytopathologie standard ont été classés comme positifs et ont fourni un diagnostic clair des métastases cancéreuses.

Résultats: 28 spécimens ont été classés comme métastatiques et 12 comme non métastatiques par cytopathologie standard. 
L'analyse FC a confirmé la présence de cellules cancéreuses du poumon dans tous les LN métastatiques (médiane $=31,10 \%(4,19 \%$ $-70,12 \%)$ ). Nous avons constaté que parmi 12 spécimens classés comme non métastatiques, des cellules cancéreuses du poumon ont été trouvées dans 7 spécimens (médian $=1,66 \%(0,68 \%-5,00 \%)$ ). Parmi ces sept patients, quatre étaient au stade II du CPNPC et trois au stade I du CPNPC.

Conclusion: L'identification des cellules cancéreuses du poumon dans les EBUS/TBNA spécimens à l'aide de FC est faisable et pourrait encore aider à la détection des métastases des ganglions lymphatiques. S’ils sont confirmés prospectivement, ces résultats peuvent avoir une valeur significative pour le paradigme de stadification du CPNPC.

Conflits d'intérêt: Rien à déclarer

\section{P129 \\ COVID-19: Des Réponses Individuelles Pour des Stratégies Collectives (Pologne Et France) - Peur}

\section{Christelle Langrand, Natalia Soja-Kukieła, Aldona Katarzyna Jankowska}

Université Nicolas-Copernic, Collegium Medicum, Equipe de recherche et de didactique en communication médicale; Université Nicolas-Copernic, Secrétariat Initiative

d'excellence - centre de recherches

Introduction: Face à la pandémie de COVID-19, des stratégies collectives sont mises en place dans le cadre de la prévention dans chaque pays selon des modalités différentes. L'un de leurs leviers est la peur engendrée par le virus SARS-CoV-2. Elles ne seront efficaces que si elles interagissent avec des réponses individuelles. Il semblerait alors pertinent de dessiner le profil-type d'une personne ayant peur des conséquences de la COVID-19 aussi bien au titre personnel que national, ce qui est l'objectif de notre étude menée au cours de l'été 2020 en France et en Pologne.

Méthode: Deux sondages originaux et identiques ont été conçus pour recueillir des données socio-démographiques et médicales ainsi que le taux de personnes ayant peur des conséquences de cette maladie. 117 personnes vivant en Pologne et 147 en France ont répondu aux questionnaires, ce qui a permis d'analyser statistiquement ces réponses à l'aide du test du Khicarré et du test $t$ de Student $(\mathrm{p}<0,05)$.

Résultats: Quel que soit le pays, environ $80 \%$ des personnes interrogées affirment avoir peur. Ce taux ne dépend statistiquement que de certains critères liés à l'état de santé des personnes interrogées et de façon fiable du niveau de formation en Pologne (plus ce niveau est élevé et moins les gens ont peur, $p=0,001$ ). Des différences statistiquement significatives existent entre les deux panels aux niveaux des critères déjà cités.

Conclusion: Aucun traitement pleinement efficace n'a encore été trouvé et les campagnes de vaccination se dérouleront sur plusieurs mois. Par conséquent, des stratégies collectives de prévention devraient être adoptées afin de maintenir un juste équilibre entre peur et responsabilité individuelle afin de réduire la propagation du SARS-CoV-2. Pour cela, il est crucial de correctement éduquer le grand public grâce à des communications médicale et politique efficaces.

Conflits d'intérêt: Rien à déclarer

\section{P130 \\ Clinical Feature of Community Acquired Pneumonia in Smokers}

\section{E. Ben Jemia, K. Euchi, H. Zaibi, H. Ouertani, A. Naaroura, J. Ben Amar, H. Aouina}

Pulmonary department, Charles Nicolle Hospital, Tunis Medical Faculty of Tunis, University of Tunis El Manar, Tunisia

Introduction: Smoking triggers an immunologic response to vascular injury, which is associated with increased levels of inflammatory markers. Evidence suggests an increased susceptibility to infection in smokers.

Aim: To bring out the correlation exsiting beteween tobacco and community acquired pneumonia features (CAP).

Methods: We performed a retrospective study using records of hospitalized patients for CAP between January 2018 and December 2019.

G1: smokers hospitalized for CAP and G2: Non-smokers hospitalized for CAP.

Results: We included 43 patients, with sex ratio at 0.6 . Twenty five patients $(58 \%)$ were smokers. Median age was 62 years in G1 vs 58 years in $\mathrm{G} 2(\mathrm{p}=0.4, \mathrm{r}=0.1)$. PAC severity was evaluated by using CURB65 and FINE scores that were higher in G1 with a significant difference (respectively: $\mathrm{p}=0.03 ; \mathrm{r}=0.04 / \mathrm{p}=0.02 ; \mathrm{r}=0.34$ ). All patients received antibiotics. Clinical improvement was slower in G1 with no significant difference (3.7 days vs 2.7 days, $\mathrm{p}=0.2$ ). Tobacco was associated with a significatly longer hospital stay (17 days vs 12 days, $\mathrm{p}=0.01 ; \mathrm{r}=0.37$ ) and longer radiological improvement delay ( 30 days vs 15 days, $\mathrm{p}=10-3 ; \mathrm{r}=0.5$ ).

Conclusion: Smoking is associated with a more severe community acquired pneumonia with a longer course of the disease.

Disclosure: Nothing to disclose

\section{P131}

\section{Rôle du Médecin Traitant dans L'observance} Thérapeutique Chez les Adolescents Asthmatiques

\section{E. Ben Jemia, H. Zaibi, H. Ouertani, A. Allouche, S. Rejeb, S. Belkhir, J. Ben Amar, H. Aouina}

Service de pneumologie, Hôpital Charles Nicolle, Tunisie Faculté de médecine de Tunis, Université Tunis El Manar

Introduction: En pleine croissance, l'adolescent est connu pour être rebelle. Les adolescents asthmatiques ont tendance à négliger leur traitement et s'exposent ainsi à de graves complications. Le respect des instructions et des prescriptions médicales 
reste donc le paramètre clé de la prise en charge de l'asthme chez l'adolescent.

Objectifs: Evaluer le rôle du médecin traitant dans l'observance thérapeutique chez les adolescents asthmatiques.

Méthode: Étude transversale portant sur 37 patients asthmatiques âgés de moins de 18 ans, régulièrement suivis au service de pneumologie de l'hôpital Charles Nicolle de Tunis entre 2013 et 2020. Le recueil des données était fait soit par appel téléphonique soit lors de la consultation externe en utilisant le score de Morisky pour évaluer l'observance du traitement de fond.

Résultats: Trente-sept patients étaient inclus. Le sexe ratio était de 1.05. L'âge moyen était de 15.8 ans (13-18 ans). Le tabagisme était noté chez 28 patients. La principale comorbidité était l'allergie sous ses différents aspects. Vingt-neuf patients avaient de mauvaises à moyennes conditions socioéconomiques. L'asthme était bien contrôlé chez 11 patients, partiellement contrôlé chez 18 patients et non contrôlé chez 8 patients. Seulement 4 patients $(10,8 \%)$ étaient jugés de bons observants du traitement de fond de leur maladie asthmatique. L'adhérence au traitement était significativement influencée par la participation du patient au choix thérapeutique (traitement inhalé, comprimés) $(\mathrm{p}=0,007)$ et au choix du dispositif (diskus, turbuhaler.) ( $\mathrm{p}=0,001)$. La vérification de la technique d'inhalation par le médecin traitant a contribué de même à l'observance thérapeutique $(\mathrm{p}=0,01)$. Après régression logistique, seule la participation du patient au choix du dispositif était un facteur influençant l'observance thérapeutique $(\mathrm{p}=0,01)$.

Conclusion: L'asthme de l'adolescent doit constituer une entité particulière etl'alliance médecin traitant/patient est primordiale pour garantir une meilleure observance au traitement.

Conflits d'intérêt: Rien à déclarer

\section{P132 \\ Facteurs Prédictifs de la Sévérité des Exacerbations Aigues dans les Dilatations des Bronches}

\author{
H. Mrassi, I. Bachouch, N. Khezami, N. Belloumi, I. Nouira, \\ C. Habbouria, F. Chermiti, S. Fenniche
}

Service de pneumologie, Pavillon 4 de l'hôpital Abderrahmane Mami- Ariana

Introduction: Au jour d'aujourd'hui, il existe peu d'études s'intéressant au profil évolutif des dilatations des bronches (DDB), maladie respiratoire chronique dotée d'un risque important d'exacerbations aigues (EA).

But: Déterminer la fréquence et la sévérité des EA chez les patients (pts) atteints de DDB et rechercher les facteurs influençant la durée d'hospitalisation pour EA.

Patients et méthode: Etude rétrospective descriptive menée sur les dossiers des patients (pts) suivis pour DDB au pavillon 4 de l'hôpital Abderrahmane Mami [2017-2020].

Résultats: Cent-deux pts (100\% hommes) étaient inclus. L'âge moyen était de 65 ans. Les DDB post-tuberculeuses (post-TB) étaient les plus fréquentes (39\%). On avait dénombré 142 EA nécessitant l'hospitalisation; un tiers des pts avait au moins 2 EA sur l'année. Une insuffisance respiratoire aiguë était notée dans
$45 \%$ des cas. Le nombre moyen annuel d'EA avec hospitalisation, était plus important en cas de DDB post-TB $(\mathrm{p}=0,03)$. La durée d'hospitalisation (D) était de $13 \pm 9$ jours en moyenne. $D$ était plus prolongée en présence de comorbidités cardiovasculaires $(\mathrm{p}=0,04)$, respiratoires $(\mathrm{p}=0,02)$ et d'insuffisance pondérale $(\mathrm{p}=\mathrm{NS})$. De plus, D était plus importante en cas de DDB étendues (nombre de lobes atteints $>3)(\mathrm{p}=0,03)$, d'infection par le Pseudomonas Aeroginusa (PA) $(\mathrm{p}=0,04)$ et d'anémie $(\mathrm{p}=\mathrm{NS})$. Par ailleurs, une durée d'hospitalisation prolongée n'était corrélée ni à la durée de l'évolution des $\mathrm{DDB}$, ni à l'intoxication tabagique en paquetsannées, ni au VEMS.

Conclusion: Le terrain du pt, l'étendue des lésions et l'infection par le PA seraient prédictifs d'une hospitalisation prolongée au cours des EA de DDB.

Conflits d'intérêt: Rien à déclarer

\section{P133 \\ L'élaboration, Par Une Démarche en Trois Étapes, de Recommandations Pour la Prise en Charge des Malades Hospitalisés Pour Pneumonies Communautaires. Difficultés et Propositions}

\author{
Zeina Aoun-Bacha, Céline Baaklini, Georges Khayat, \\ Carine Harmouche, Diana Sassine, \\ Carole Youakim
}

Société Libanaise de Pneumologie - Beyrouth - Liban

Introduction: La prise en charge des malades hospitalisés pour pneumonie communautaire (PC) semble être hétérogène au Liban avec, de l'avis - subjectif - de nombreux pneumologues, un abus de prescription antibiotique. Notre objectif était de clarifier et d'améliorer la prise en charge de ces malades par l'élaboration de recommandations tenant compte des spécificités nationales.

Méthodes: Les trois étapes consistaient en:

(1) Un état des lieux des pratiques professionnelles, le plus large possible, qui étudierait les dossiers de malades hospitalisés, durant un an, pour PC (critères ERS) dans le plus grand nombre d'hôpitaux, et en relèverait les données démographiques, les comorbidités, les explorations pratiquées et leurs résultats, les traitements administrés et l'évolution des malades.

(2) Une analyse des données serait faite avec les pneumologues impliqués.

(3) Des recommandations nationales seraient rédigées, proposées et validées par les mêmes pneumologues.

Résultats: L'accès aux dossiers a été limité à cinq hôpitaux par la présence de nombreux obstacles. Les dossiers de 215 hommes et 173 femmes ont été revus. La mortalité était de 5,15\% avec un risque plus élevé chez les malades $>80$ ans $(\mathrm{OR}=3,07$; [IC1,24:7,61], ou atteints de cancer $(\mathrm{OR}=3,98$; [IC2,09:7,59], de fausses routes $(\mathrm{OR}=5,47 ;[\mathrm{IC} 1,64: 18,24]$ ou d'atteintes neurologiques $(\mathrm{OR}=2,77$; [IC1,02:7,56].

Les fréquences des explorations pratiquées étaient: la formule numération $(99,5 \%)$, la CRP $(98,5 \%)$, les hémocultures $(60,8 \%)$, la culture des crachats $(53,3 \%)$, la procalcitonine $(21 \%)$, les prélèvements endoscopiques $(8,8 \%)$, la recherche d'antigènes 
urinaires pour Legionella $(8 \%)$ ou pneumocoque $(6,4 \%)$ et le prélèvement nasopharyngée $(6,2 \%)$.

La prescription antibiotique était empirique (387/388). 160 $(41,23 \%)$ d'entre elles ne correspondaient à aucune des recommandations (ERS, ATS/IDSA, SPLF).

L'analyse des données a insisté sur plusieurs points: a-Les explorations tiennent compte de l'évaluation clinique du malade sans être pratiquées systématiquement ; b-Certaines explorations ne sont pas, ou sont difficilement, disponibles dans certains hôpitaux ; c-La prise en charge est hétérogène selon le médecin traitant ; d-L'usage d'antibiotiques est abusif, en relation avec l'anxiété du médecin, du malade et/ou de son entourage.

Conclusion: Les recommandations élaborées mettront l'accent sur: a-L'amélioration de la tenue des dossiers ; b- La standardisation des attitudes; $\mathrm{c}$-La simplification des recommandations internationales en les adaptant à nos spécificités locales ; d-La nécessité de mener des études prospectives pour mieux caractériser les pneumonies communautaires au LIban, et y adapter les recommandations.

Conflits d'intérêt: Rien à déclarer

\section{P134 \\ Pleural Tuberculosis: Comparative Study between Adult Form and Infantile Form}

\section{Baili Hassène, Hamdi Besma, Khalfallah Ikbel, Louhaichi Sabrine, Marzouki Safa, Kaabi Line, Fidha Sabrine, Jebali Rihab Ammar Jamel, Hamzaoui Agnès}

Service de pneumologie B, Hôpital A. Mami, Tunisie

Introduction: Pleural tuberculosis (pTB) is one of the most common location of extra-pulmonary tuberculosis. The difficulty of bacteriological confirmation due to the low sensitivity of the used tools makes this entity a problematic disease in children and adults.

Aim: Compare the clinical and para-clinical characteristics and diagnostic tools of pTB in children and adults.

Method: Retrospective study including 76 patients hospitalized for pTB including 27 children and 49 adults between 2009 and 2019. The adults were all women due to the recruitment of our department. Different diagnostic tools were performed: radiological examination, pleural puncture, biopsy, bacteriological study, PCR. The clinical and para-clinical data were analyzed and compared between the two groups.

Results: The mean age was 12.4 years in children and 40 years in adults. Tuberculosis contagion was found in $50 \%$ of children vs. $24 \%$ for adults. Only $40 \%$ of adults vs $92 \%$ of children were vaccinated with BCG. In each group 2 cases had a family history of Tbc. Merely 23 children were symptomatic with a delay of onset of symptoms $>=1$ month in $37 \%$ vs 48 adults including $45.8 \%$. General signs were more present in children; $65 \%$ vs. $41 \%$ of adults ( $p>0.43$ ). In addition, respiratory symptoms were more obvious in adults; $53.5 \%$ vs. $37 \%(\mathrm{p}=0.06)$. Tuberculin IDR was positive in $59 \%$ of children vs only $14 \%$ of adults $(p=0.02)$. Chest $x$-ray revealed low-abundance pleural effusion in the majority of chil- dren, but $44 \%$ of adults had large pleural effusions abundance. The use of chest imaging (chest ultrasound/CT-Scan) was necessary in less than $1 / 3$ of the cases in both groups. Pleural puncture was performed in $88.9 \%$ of children vs $100 \%$ in adults revealing lymphocytic exudate in $95 \%$ of children vs $98 \%$ of adults. PCR of koch bacillus (BK) in pleural fluid was positive in $16.4 \%$ of children vs $10.2 \%$ of adults $(\mathrm{p}=0.4)$. Pleural biopsy was performed in 25 children vs. 45 adults confirming the diagnosis in 50\% and 75\% of cases respectively $(\mathrm{p}=0.06)$. Surgery was indicated in 2 cases in 2 groups. The diagnosis was made on the basis of arguments in $33 \%$ of children and only 1 case in adults $(p=0.02)$. The outcome was favorable with HRZE treatment. Only $14.8 \%$ of children vs. $10.2 \%$ of adults had side effects. No recurrence has been objectified in children.

Conclusion: The characteristics of pTB in adults and children are almost similar. Diagnosis and management in children constitute a real challenge for clinicians, requiring new optimal strategies.

Disclosure: Nothing to disclose

\section{P135 \\ Résultats Préliminaires D'un Suivi Respiratoire D’une Cohorte des Patients Ayant Présenté Une Pneumonie À Sars-Cov-2 Antananarivo Madagascar}

L. Rebasy' ${ }^{1}$, RM. Andrianasolo ${ }^{2}$, ID. Rakotondrabe ${ }^{1}$, NLE. Rafitoharison ${ }^{1}$, AH. Ranivoson ${ }^{3}$, K. Ravahatra ${ }^{4}$, Tiaray HM ${ }^{1}$, JR. Rakotomizao ${ }^{1}$, JL. Rakotoson ${ }^{1}$, RN. Raharimanana ${ }^{4}$

'Unité de Soins, de Formations et de Recherches (USFR) de Pneumologie Centre Hospitalier Universitaire, Antananarivo, Madagascar, ${ }^{2}$ Institut Nationale de la Santé Publique et communautaire Antananarivo, Madagascar, ${ }^{3}$ Centre Hospitalier Universitaire Mère Enfant Tsaralalana Antananarivo Madagascar ${ }^{4}$ Service de Pneumologie Centre Hospitalier Universitaire,

Antananarivo, Madagascar

Introduction: Après une infection à COVID-19, un bilan fonctionnel respiratoire et un suivi personnalisé sont préconisés. Nous rapportons les résultats préliminaires d'un suivi spécialisé en pneumologie d'une cohorte de patients ayant présenté une pneumonie à SARS-Cov2. L'objectif est de décrire l'évolution clinicoradiologique à un mois.

Méthode: Il s'agit d'une étude de cohorte prospective de patients recrutés entre juillet et décembre 2020 à Antananarivo dans les centres de prise en charge du covid-19, et ayant présenté une forme modérée à critique de la maladie à coronavirus 2019 à la phase aiguë.

Résultats: Parmi les 67 patients, 38 étaient des hommes et 29 des femmes. L'âge moyen était de 54 ans $(\mathrm{ET}=14)$. L'essoufflement était présent chez 38 patients $(56.7 \%)$. La douleur thoracique était surtout retrouvée chez les patients avec $10 \%$ d'atteinte au scanner. La polypnée était présente chez 12 patients (18\%), elle était corrélée avec une atteinte de plus de $50 \%$ au scanner thoracique $(\mathrm{p}=0.02)$. Le scanner thoracique était pathologique dans $92.5 \%$ des cas dont l'atteinte pulmonaire entre 10 et $50 \%$ était la plus représentée
SSP/SSTS - EFP

Joint Annual Conference 2021 
(37,3\%). A un mois, la persistance des signes respiratoires avec quasi les mêmes proportions de patients qu'au départ était constatée: essoufflement $53 \%$, douleur thoracique $30 \%$, polypnée $18 \%$. Une désaturation moins de $90 \%$ était retrouvé chez 20 patients $(29.8 \%)$. Le scanner à un mois ne retrouve plus d'atteinte de plus de $50 \%$ au scanner par contre 26 (39\%) restait pathologique.

Conclusion: La persistance des symptômes respiratoires et des anomalies scannographiques à un mois justifient la poursuite du suivi jusqu'à 6 mois afin d'une part d'éviter les séquelles respiratoires, et d'autre part permettre de mieux comprendre le potentiel d'évolution des lésions

Conflits d'intérêt: Rien à déclarer

\section{P136 \\ Impact du Traitement Par CPAP Sur le Risque Cardio-Vasculaire des Patients Ayant un Syndrome D’apnées Obstructives du Sommeil Sévère}

\author{
S. Bouafia' , M. Loukil' ', N. Abid' ', S. Kalboussi' ${ }^{1}$, M. Ben Ali ${ }^{2}$, H. Ghrairi \\ ${ }^{1}$ Service de pneumologie, hôpital Taher Maamouri Mohamed, \\ Nabeul, ${ }^{2}$ Service d'anesthésie réanimation, hôpital Taher \\ Maamouri Mohamed, Nabeul
}

Introduction: Le syndrome d'apnées obstructives du sommeil (SAOS) touche environ $4 \%$ des hommes et $2 \%$ des femmes d'âge moyen mais beaucoup de ces patients ne sont ni diagnostiqués ni traités. Une morbidité et une mortalité cardio et cérébrovasculaires sont associées à ce syndrome. La notion de lien de causalité s'est confirmée ces dernières années sur des données épidémiologiques, expérimentales et thérapeutiques.

Méthodologie: étude prospective descriptive longitudinale intéressant des patients apnéiques suivis au service de pneumologie de l'hôpital Mohamed Taher Maamouri de Nabeul. Le risque cardio-vasculaire a été évalué chez tous les patients et ce risque a été réévaluer chez les patients traités par pression positive

Résultats: Nous avons inclus 350 patients ayant un SAOS confirmés par polygraphie ventilatoireL'âge moyen de nos patients était de 56,4 ans $\pm 12,2$ (18-88 ans). Le diabète a été noté dans 237 cas $(67,7 \%)$, l'insuffisance cardiaque dans 10 cas $(2,9 \%)$, trouble du rythme dans 21 cas (6\%), la dyslipidémie dans 92 cas $(26,3 \%)$. Le SAOS était sévère dans 158 cas $(45,1 \%)$, modérée dans 89 cas $(25,4)$ et légère dans 106 cas $(30,3 \%)$.

Le risque cardio-vasculaire a été évalué à 19,2 $\pm 17,5$ (1-97), ce risque a été évalué à $23,5 \pm 19,9$ chez les patients ayant un SAOS sévère versus 15,6 chez les patients ayant un SAOS léger et modéré $(\mathrm{P}<0,001)$. Chez les patients traités par pression positive continue avec une bonne observance thérapeutique, le risque cardio-vasculaire a été passé de $23,5 \pm 19,9$ à $12,7 \pm 10,2(\mathrm{P}<0,001)$.

Conclusion: Dépister et traiter un SAOS est le seul moyen pour diminuer la mortalité en rapport avec les comorbidités cardio-vasculaires.

Conflits d'intérêt: Rien à déclarer

\section{P137 \\ Les Facteurs Prédictifs de la Sévérité du Syndrome D’apnées Obstructives du Sommeil}

\author{
S. Kalboussi' , M. Loukil', N. Abid ' ', S. Bouafia' , M. Ben Ali ${ }^{2}$, \\ H. Ghrairi', S. Kalboussi ', M. Loukil', N. Abid' ${ }^{1}$, S. Bouafia', \\ M. Ben Ali $^{2}$, H. Ghrairi ${ }^{1}$
}

'Service de pneumologie, hôpital Mohamed Taher Maamouri, Nabeul, ${ }^{2}$ Service d'anesthésie réanimation, hôpital Mohamed Taher Maamouri, Nabeul

Introduction: le syndrome d'apnées hypopnées obstructives du sommeil (SAOS) est une pathologie associée à des comorbidités cardio-vasculaires qui augmente avec la sévérité de cette maladie.

Méthodologie: Etude prospective descriptive longitudinale sur une période allant de novembre 2016 à juin 2019 incluant 350 patients suivis dans notre service pour SAOS confirmé par un enregistrement polygraphique. Notre population a été divisée en 2 groupes: $\mathrm{G} 1$ patients ayant un SAOS sévère et $\mathrm{G} 2$ patients ayant un SAOS légère et modéré.

Résultats: L'âge moyen était de 56 ans avec des extrêmes allant de 18 à 88 ans Le SAOS était sévère dans 158 cas $(45,1 \%)$ avec IAH moyen à $51,4 / \mathrm{h}$ et un index de désaturation à $43,2 / \mathrm{h}$. Le SAOS était légère et modérée dans 192 cas $(54,9 \%)$. l'intoxication tabagique était notée dans 59 cas dans $\mathrm{G} 1$ versus 44 dans $\mathrm{G} 2(\mathrm{P}=0,05)$. La BPCO était notée dans 16 cas dans $\mathrm{G} 1$ vs 14 cas dans $\mathrm{G} 2(\mathrm{P}=0,4)$ BMI moyen était de 38,2 dans $\mathrm{G} 1$ vs 35,4 dans $\mathrm{G} 2$ ( $\mathrm{p}=0,001)$, le SAOS était plus sévère chez la femme dans 97 cas $(\mathrm{p}=0,02)$. Le diabète a été noté dans 49 cas dans G1 vs 63 cas dans $\mathrm{G} 2(\mathrm{p}=0,7)$. Le SAOS était plus sévère chez les sujets âgés avec $p=0,06$. L'étude multivariée avait montré que les facteurs prédictifs de la sévérité du SAOS était le tabagisme $(\mathrm{OR}=1,005)$ et le sexe $(\mathrm{OR}=1,003)$.

Conclusion: préciser les facteurs prédictifs de sévérité du SAOS est important afin d'agir sur ces facteurs pour améliorer le pronostic de cette maladie.

Conflits d'intérêt: Rien à déclarer

\section{P138 \\ Pneumopathies Infiltrantes Diffuses Aiguës Non Infectieuses en Réanimation: Apport du Scanner Thoracique}

Affes Meriem, Attia Monia, Khayati Rym, Kchaou Salma, Baccouche Ines, Nèji Henda, Hantous-Zannad Saoussen

Imagerie médicale - Hôpital Abderrahmen Mami de l'Ariana

Introduction: Les pneumopathies infiltrantes diffuses (PID) aiguës regroupent un ensemble hétérogène d'affections respiratoires caractérisées par une atteinte diffuse du parenchyme pulmonaire intéressant le secteur interstitiel et/ou les voies aériennes distales. Elles posent un problème de diagnostic étiologique compte-tenu de leur diversité. L'objectif de notre étude était d'évaluer le rôle du scanner dans le diagnostic étiologique des PID aiguës non infectieuses. 
Méthode: Étude rétrospective étendue sur cinq ans incluant les patients hospitalisés pour une détresse respiratoire aiguë, et chez qui le scanner thoracique avait conclu à une PID aiguë. Nous n'avons pas inclus les PID d'origine infectieuse. Nous avons procédé à une analyse des patterns ou groupements des lésions élémentaires tomodensitométriques, puis nous avons confronté nos conclusions au diagnostic final retenu.

Résultats: Nous avons inclus 35 patients avec un âge moyen de 51 ans. Les étiologies retenues de la PID aiguë étaient dominées par l'hémorragie alvéolaire (31\%) puis par l'œdème aigu du poumon (OAP) hémodynamique (23\%) et les exacerbations de PID chronique (17\%).

L'hémorragie alvéolaire a été évoquée chez 10 patients parmi 11, l'OAP hémodynamique dans sept cas parmi huit et l'exacerbation de PID chronique dans cinq cas parmi six. La pneumopathie aiguë à éosinophiles n'a été évoquée dans aucun cas. Les signes élémentaires les plus fréquents étaient les plages d’hyperdensités en "verre dépoli » $(90 \%$ des cas) et les condensations ( $74 \%$ des patients). Une distribution centrale des lésions était corrélée au diagnostic d'hémorragie alvéolaire $(\mathrm{p}=0,01)$. L'épaississement des lignes septales $(\mathrm{p}=0,04)$ et l'épaississement péribronchovasculaire $(\mathrm{p}=0,017)$ étaient significativement plus fréquents au cours de l'œdème aiguë pulmonaire.

Conclusion: Devant une PID aiguë en milieu de réanimation, la confrontation des signes élémentaires tomodensitométriques et de leur distribution, aux données anamnestiques, cliniques et paracliniques permet le plus souvent d'aboutir au diagnostic étiologique.

Conflits d'intérêt: Rien à déclarer

\section{P139 \\ Les Pleurésies Néoplasiques: Profil Épidémiologique, Clinique, Paraclinique, et Étiologique}

\section{M. ijim, S. aitbatahar, L. amro}

Service de pneumologie. Hôpital Arrazi, CHU Mohammed VI, FMPM, UCA, Marrakech, Maroc

Introduction: La pleurésie néoplasique traduit la présence de cellules tumorales dans l'espace pleural, une des complications les plus fréquentes de la maladie cancéreuse.

Méthode: Nous rapportons une étude rétrospective portant sur 28 cas porteurs de pleurésie néoplasique colligés dans notre service entre Janvier 2019 et février 2020.

Résultats: Il s'agit de 12 hommes et 16 femmes, l'âge moyen était de 58 ans. Le diagnostic du cancer primitif existait déjà dans 8 cas soit $28.6 \%$, la pleurésie était révélatrice dans 20 cas (71.4\%), Le tabagisme était retrouvé dans 10 cas soit $35.7 \%$. Les symptômes étaient dominés par: la dyspnée (100\%), la douleur thoracique (100\%), une altération de l'état général (92\%). Les malades présentaient un syndrome d'épanchement liquidien. L'aspect du liquide était sérohématique dans 16 cas. La radiographie du thorax l'aspect d'une pleurésie dont l'abondance était grande dans 24 cas soit $85.7 \%$, et moyenne dans 4 cas (14.2\%). Le coté de l'épanchement était droit dans 10 cas (35.7\%), il était gauche dans 16 cas (57.1\%) et bilatéral dans 2 cas cas. On avait recours au scanner thoracique dans $64.3 \%$ des cas. La ponction biopsie pleurale faite chez tous les patients, la fibroscopie bronchique dans 12 cas (42.9\%). La ponction pleurale a confirmé le diagnostic dans 8 cas (28.6\%), la biopsie pleurale dans 12 cas (42.8\%). Une localisation pleurale d'un cancer broncho-pulmonaire était diagnostiqué dans 6 cas, lymphome non hodgkinien dans 4 cas, adénocarcinome ovarien dans 4 cas, 2 cas de myélome multiple, 1 cas d'adénocarcinome gastrique, et 1 cas de mésothelium sarcomatoide. Le traitement était palliatif dans presque tous les cas.

Conclusion: La pleurésie néoplasique peut être révélatrice d'un cancer dont la détermination du primitif est difficile dans des cas, Leur origine principale est le cancer du poumon chez l'homme et les cancers pulmonaires et pelviens chez la femme.

Conflits d'intérêt: Rien à déclarer

\section{P140 \\ Asthme et Obésité}

\section{Ijim, S. Aitbatahar, L. Amro}

Service de pneumologie. Hôpital Arrazi, CHU Mohammed VI, FMPM, UCA, Marrakech, Maroc

Introduction: L'asthme est une maladie respiratoire fréquente, grave et parfois mortelle. Elle constitue un problème de santé publique, et il y'a plusieurs facteurs qui influencent le contrôle de l'asthme. But du travail: Le but de notre travail est de déterminer si l'obésité serait un facteur de son mauvais contrôle.

Méthodes: Nous rapportons une étude rétrospective incluant les patients asthmatiques obèses, dont l'indice de masse corporelle (IMC) est supérieur à $30 \mathrm{~kg} / \mathrm{m} 2$, suivis en consultation d'allergologie du service entre juin 2018 et décembre 2019.

Résultats: Nous avons colligé 23 femmes et 6 hommes âgés entre 20 et 68 ans (moyenne d'âge: 43.6 ans). L'HTA est retrouvé dans 8 cas, le diabète dans 6 cas et une dysthroidie dans 2 cas. L'asthme était intermittent dans $51.7 \%$, persistant léger dans $24.2 \%$, persistant modéré dans $17.2 \%$ et persistant sévère dans 6.9\%. Le reflux gastro-œsophagien était retrouvé dans $41.4 \%$ des cas, une rhinite d'allure allergique dans $48.3 \%$ des cas, une conjonctivite dans $10.3 \%$ des cas, Le traitement préconisé repose sur les corticoïdes inhalés et les béta2mimétiques de longue durée d'action, associés aux xanthiniques dans quatre cas, aux antihistaminiques et corticoïdes nasaux dans 13 cas et aux inhibiteurs de la pompe à proton dans 11 cas. L'asthme est bien contrôlé dans $58.6 \%$ des cas, partiellement contrôlé dans $27.6 \%$ des cas et non contrôlé dans $13.8 \%$ des cas. La courbe débit volume faite chez tous nos patients a objectivé un trouble ventilatoire obstructif dans $86.2 \%$ et mixte dans $13.8 \%$.

Conclusion: l'obésité pourrait être un facteur de mauvais contrôle de l'asthme et qu'une prise en charge multidisciplinaire pourrait être bénéfique afin de garantir une meilleure approche thérapeutique

Conflits d'intérêt: Rien à déclarer 


\section{P141}

\section{Profil Fonctionnel Respiratoire de la Sarcoïdose}

\section{Aazri, S. Ait batahar, L. Amro}

Service de pneumologie. Hôpital Arrazi, CHU Mohammed VI, Marrakech, Maroc

Introduction: La sarcoïdose est une granulomatose systémique d'étiologie inconnue, ayant comme localisation privilégiée, le poumon et le système lymphatique. Elle peut retentir sur la fonction respiratoire et aboutir à l'insuffisance respiratoire chronique. L'exploration fonctionnelle respiratoire permet d'évaluer le retentissement sur la fonction respiratoire et d'apprécier la réponse aux différents traitements.

Le but de notre travail: Déterminer le profil fonctionnel respiratoire des patients atteints de sarcoïdose thoracique.

Matériel et méthode: Nous rapportons une étude rétrospective incluant les cas de sarcoïdose thoracique ayant bénéficier d'une pléthysmographie et d'une gazométrie artérielle au service de pneumologie du CHU Mohamed VI de Marrakech entre janvier 2019 et Janvier 2021.

Résultats: Seize cas ont été colliges. L'âge moyen était de 55 ans, dont 15 femmes $(93,75 \%)$ et 1 homme $(6,25 \%)$. La dyspnée était retrouvée dans $75 \%$ des cas, la toux dans $62,5 \%$ des cas et les arthralgies dans $50 \%$ des cas. La sarcoïdose était de type I dans $6,25 \%$ des cas, de type II dans $31,25 \%$ des cas, de type III dans $56,25 \%$ des cas et de type IV dans 6,25\% des cas. La pléthysmographie avait objectivé un trouble ventilatoire restrictif dans $31,2 \%$ des cas, mixte dans $18,7 \%$ des cas, une atteinte des petites voies aériennes dans $18,7 \%$ des cas et un trouble ventilatoire obstructif dans $6,2 \%$ des cas. La pléthysmographie était normale dans $25 \%$ des cas. La gazométrie artérielle a objectivé une hypoxémie dans 43,7\% des cas et un taux de $12,5 \%$ pour l'hypercapnie et l'acidose. La gazométrie artérielle était normale dans $31,2 \%$ des cas. Une corticothérapie inhalée en association avec un bronchodilatateur de longue durée d'action a été prescrite chez 4 patients (25\%), quant à la corticothérapie seule, elle a été prescrite chez 3 patients $(18,75 \%)$. Un traitement par corticothérapie systémique au long court avec traitement adjuvant était instauré chez 14 patients $(87,5 \%)$. L'évolution était marquée par l'amélioration clinique dans 15 cas (93,75\%), A noter un cas de fibrose dans (6,25\%) pour lequel l'oxygénothérapie au long court était indiquée. Conclusion Bien qu'elle soit une pathologie bénigne, la sarcoïdose peut s'accompagner d'un retentissement respiratoire.

Conclusion: L'exploration fonctionnelle respiratoire est d'un grand intérêt dans l'évaluation et la surveillance de l'évolution de la pathologie ainsi que pour guider la décision thérapeutique. Bien que le trouble ventilatoire restrictif soit la principale conséquence fonctionnelle respiratoire, l'attente obstructif associée ou isolée peut aussi s'observer au cours de cette pathologie.

Conflits d'intérêt: Rien à déclarer

\section{P142 \\ L'effet Psychologique de la Pandémie du COVID 19 Sur le Personnel du CHU Mohammed VI de Marrakech}

\section{Ijim, S. Aitbatahar, L. Amro}

Service de pneumologie. Hôpital Arrazi, CHU Mohammed VI, FMPM, UCA, Marrakech, Maroc

Introduction: Les professionnels de la santé ont subi une pression très importante durant cette pandémie du COVID-19. On vise à évaluer l'impact psychologique sur les professionnels du CHU Mohammed VI de Marrakech. Du 31 Mai au 22 juin 2020.

Méthode: C'est une étude transversale utilisant un questionnaire mesurant; l'échelle d'impact de l'événement - révisée (IES$\mathrm{R})$, Une analyse factorielle exploratoire et bivarié a été réalisée pour exploiter les résultats.

Résultats: 117 personnes qui ont répondu, $66.67 \%$ étaient des femmes, l'âge moyen:30 ans (de 25 à 60 ans), 46,15\% étaient médecins, $46.15 \%$ étaient des infirmiers, 15 personnes avec des antécédents psychiatriques, $25.65 \%$ des participants vivaient seul, Parmi les participants à l'étude, 93 soit $79.46 \%$ ont travaillé directement avec des patients atteints par le covid 19, le nombre des personnes qui ont changé leur poste d'affectation était de 54 soit $46.15 \%$, en moyenne le nombre d'heures de travail était $36 \mathrm{~h}$ par semaine par personne, la rémunération était jugée insatisfaisante par $82.05 \%$ des personnes, 58.97 ont que estimé la disponibilité des équipements de protection est satisfaisante. 15.39\%; ont gardés une activité physique régulière, $10.26 \%$ ont gardé un contact physique proche avec leur familles, le degré du stress en global était jugé très important par $66.67 \%$ des gens, $53.84 \%$ ont présenté une insomnie, $12.82 \%$; une agressivité, la somatisation a été présenté par 9 personnes (7.69\%), les cauchemars par 9 d'autres (7.69\%) et la peur intense par 3 personnes. 9 personnes qui ont déjà consulté la cellule psychiatrique d'écoute. Le score de l'IES-R est calculé à 41.94 soit en faveur d'un trouble de stress post traumatique répandu dans cet échantillon, avec un score d'évitement à 12,76, intrusion de souvenirs à 18.58 et d'hyperactivité à 10.58 .

Conclusion: Les impacts psychologiques indésirables sont répandus parmi nos professionnels. Le dépistage et l'élaboration de mesures préventives correspondantes seraient bénéfiques.

Conflits d'intérêt: Rien à déclarer 


\section{P143 \\ Asthme de L'enfant à Antananarivo: Pratique des Médecins de Ville}

N.I.E. Rafitoharson' , A.H. Ranivoson' ', L. Rebasy', Z.A. Randriananahirana ${ }^{1}$, K. Ravahatra ${ }^{1}$, I.D. Rakotondrabe ${ }^{1}$, H.M. Tiaray ${ }^{1}$, J. Rakotomizao ', J.L. Rakotoson ${ }^{2}$, R.N Raharimanana ${ }^{1}$

${ }^{1}$ Université d'Antananarivo Madagascar, ${ }^{2}$ Université de Fianarantsoa Madagascar

Introduction: Une prise en charge pré-hospitalière inadéquate des enfants asthmatiques reste constatée vu les recours fréquents aux urgences pour exacerbations d'asthme. L'objectifétait d'évaluer les connaissances et les pratiques des médecins sur la prise en charge de l'asthme de l'enfant.

Méthodes: Une étude transversale, descriptive et analytique à partir de questionnaires a été menée auprès de 96 médecins généralistes exerçant à Antananarivo.

Résultats: Soixante-sept médecins $(69,8 \%)$ ont consenti à participer à l'étude. Les connaissances sur l'asthme étaient bonnes $(58,2 \%)$ à moyennes $(40,3 \%)$. Les éléments de diagnostic étaient basés sur la clinique dans plus de $86 \%$ des cas et la spirométrie dans moins de la moitié des cas. La prise en charge était insuffisante dans $89 \%$ des cas. Des médicaments non recommandés tenaient leur place dans les prescriptions (30\% à 64\%). Les scores de connaissance et de prise en charge augmentaient avec le jeune âge du médecin $(\mathrm{p}=0,001)$.

Conclusion: La mise à disponibilité des outils diagnostiques et des formations médicales continues s'avèreraient nécessaires.

Conflits d'intérêt: Rien à déclarer

\section{P144 \\ Les Biopsies Thoraciques Écho-Guidées en Pratique Pneumologique en Algérie}

\section{MR. Selmani, A. Abderraouf, S. Hamoud, A. Djebbar}

Pneumo-phtisiologie - EPH Batna

Introduction: Classiquement les biopsies des lésions pulmonaires périphériques sont l'apanage du radiologue et sont souvent guidées par la tomodensitométrie. Désormais, avec l'augmentation accrue de la fréquence des nodules pulmonaires le pneumologue est appelé à jouer un rôle dans leurs diagnostic, notamment par la réalisation de biopsies écho-guidées.

Objectif: Evaluer la rentabilité diagnostique des biopsies thoraciques écho-guidées (BTEG) réalisées par le pneumologue et déterminer les facteurs influençant la rentabilité et les complications.

Méthode: Nous avons mené une étude mono-centrique prospective incluant 149 BTEG réalisées chez 143 patients sur une période allant de mars 2016 à juillet 2019.

Résultat: Les biopsies ont été complètement procédées chez 143 patients ( 107 lésions pulmonaires, 29 lésions pleuro-pariétales, 7 lésions médiastinales) et six patients ont subi une seconde biopsie de la même lésion qui était exclusivement pulmonaire. La performance globale des BTEG est de 77,2\%. La sensibilité et l'efficacité diagnostique des lésions malignes est de $74 \%$ et $76 \%$ respectivement. Le rendement est plus élevé lorsque l'opérateur a plus de 120 procédures à son actif $(\mathrm{p}=0,004)$. Nous avons recensé 27 complications (12 hémoptysies, 9 pneumothorax et 6 réactions vagales). L'analyse statistique montre que la biopsie d'un site pulmonaire $(\mathrm{p}=0,016)$ et un diamètre de la lésion $<30 \mathrm{~mm}(\mathrm{RR}=3,42$; IC 95\% $1,14-0,27 \mathrm{p}=0,048)$ sont associés à un risque de survenue d'hémoptysie. Enfin la procédure est bien tolérée avec une douleur rapportée par $36,2 \%$ des patients et un temps de procédure moyen de 15,7 minutes.

Conclusion: La biopsie écho-guidée des lésions thoraciques périphériques réalisée par le pneumologue est une technique simple pourvue d'un rendement acceptable avec peu de complications en assurant un confort au patient. Toutefois, un bon rendement de la procédure reste tributaire de l'expérience de l'opérateur et une taille lésionnelle $<$ à $3 \mathrm{~cm}$ serait un facteur de risque d'hémoptysie.

Conflits d'intérêt: Rien à déclarer

\section{P145 \\ Prévalence des Pathologies Respiratoires Chroniques Chez les Patients Hospitalisés Pour COVID 19 au Burkina Faso}

\begin{abstract}
Abdoul Risgou Ouedraogo ${ }^{1}$, Ghislain Bougma ${ }^{2}$, Adama Sourabie ${ }^{3}$, Guy Alain Ouedraogo ${ }^{4}$, Christian J. Minougou', Amed Hema², Kadiatou Boncoungou ${ }^{2}$, Georges Ouedraogo ${ }^{2}$, Flavien Kabore ${ }^{5}$, Martial Ouedraogo ${ }^{2}$

${ }^{1}$ Service de Pneumologie, Centre Hospitalier Universitaire de Tengandogo. Ouagadougou - Burkina Faso, ${ }^{2}$ Service de Pneumologie, Centre Hospitalier Universitaire Yalgado Ouedraogo. Ouagadougou - Burkina Faso, ${ }^{3}$ Service de Pneumologie, Centre Hospitalier Universitaire Souro Sanou. Ouagadougou - Burkina Faso, ${ }^{4}$ Service de maladies infectieuses et tropicales du Centre Hospitalier Universitaire Régional de Ouahigouya; Ouahigouya - Burkina Faso, ${ }^{5}$ Service de Réanimation, Centre Hospitalier Universitaire de Tengandogo. Ouagadougou - Burkina Faso
\end{abstract}

Introduction: Les patients avec antécédent de pathologies respiratoires chroniques sont à risques de développer des formes graves de la COVID-19. Bien qu'il y ait eu plusieurs études sur les facteurs pronostiques de sa forme sévère, aucune étude n'a été menée sur la prévalence des pathologies respiratoires chroniques chez les patients hospitalisés pour COVID 19 au Burkina Faso.

Méthode: Il s'est agi d'une étude transversale à collecte rétrospective portant sur la période du 09 Mars au 09 Juin 2020 à Ouagadougou, Burkina Faso et qui a concerné 456 patients atteints de COVID-19.

Résultats: La moyenne d'âge des patients était de 43,52 \pm 17,75 ans [0,58-93] et 292 patients $(292 / 456$ [64,0\%]) étaient de sexe masculin. La prévalence des pathologies respiratoires chroniques
SSP/SSTS - EFP

Joint Annual Conference 2021 
était de 5,92\% (27/456), classées troisième derrière l'HTA (99/456; $21,7 \%)$ et le diabète $(38 / 456 ; 8,3 \%)$. Parmi les patients présentant un antécédent de pathologie respiratoire chronique, 23/27 (85,2\%) étaient asthmatiques, $2 / 27$ (7,4\%) présentaient une pneumopathie séquellaire post tuberculeuse, $1 / 27(3,7 \%)$ une pleurésie enkystée chronique (d'origine non précisée) et $1 / 27$ un syndrome d'apnées du sommeil $(3,7 \%)$. Ces antécédents de pathologie respiratoire chronique étaient associés à d'autres comorbidités telles que l'HTA $(3 / 27)$, le diabète $(2 / 27)$ l'insuffisance rénale chronique (1/27) et le VIH (1/27). Au cours de l'hospitalisation 8/27 (29,6\%) de ces patients ont présenté une forme grave de la COVID-19 avec détresse respiratoire aigu et un en est décédé $(1 / 27 ; 3,7 \%)$.

Conclusion: Les pathologies respiratoires chroniques occupaient la troisième place des comorbidités chez les patients atteints de COVID 19 dans cette étude et étaient dominée par l'asthme. Une surveillance étroite de ces patients à haut risque peut permettre de réduire la létalité.

Conflits d'intérêt: Rien à déclarer

\section{P146 \\ Suivi Thérapeutique des Patients Apnéiques Sous Ventilation à Pression Positive Continue au Burkina Faso}

\begin{abstract}
Abdoul Risgou Ouedraogo' ${ }^{1}$ Edem Komlan Kunakey ${ }^{2}$, Adama Sourabie ${ }^{3}$, Amento Stéphane Adambounou 4 , Guy Alain Ouedraogo ${ }^{5}$, Kadiatou Boncoungou ${ }^{2}$, Arnaud Tiendrebeogo 2', Georges Ouedraogo², Gisèle Badoum², Martial Ouedraogo ${ }^{2}$

${ }^{1}$ Service de Pneumologie, Centre Hospitalier Universitaire de Tengandogo. Ouagadougou - Burkina Faso, ${ }^{2}$ Service de Pneumologie, Centre Hospitalier Universitaire Yalgado Ouedraogo. Ouagadougou - Burkina Faso, ${ }^{3}$ Service de Pneumologie, Centre Hospitalier Universitaire Souro Sanou - Burkina Faso, ${ }^{4}$ Service de Pneumologie, Centre Hospitalier Universitaire Sylvanus Olympio, Lomé - Togo, ${ }^{5}$ Service de Pneumologie, Centre Hospitalier Régional de Ouahigouya - Burkina Faso
\end{abstract}

Introduction: Le syndrome d'apnées obstructives du sommeil (SAOS) constitue un problème de santé publique. La ventilation par pression positive continue (PPC) est le traitement de référence. L'efficacité de ce traitement est conditionnée par la qualité de son observance et le suivi des patients. Il existe peu d'études sur le sujet en Afrique subsaharienne. D'où l'objet de cette étude qui a pour but de décrire le suivi thérapeutique des patients apnéiques sous ventilation à pression positive continue au Burkina Faso.

Méthode: Il s'est agi d'une étude transversale qui a concerné les patients sous PPC suivis pour SAOS dans les centres hospitaliers publics et privés impliqués dans la prise en charge des patients apnéiques au Burkina Faso du 1er Juin 2017 au 31 Août 2020.

Résultats: la PPC a été indiquée chez 85/150 (56,7\%) patients apnéiques répertoriés. 48/85 (56,5\%) patients ont pu l'acquérir.
Parmi ces 48 patients, 33 ont accepté participé à l'étude. Les antécédents étaient dominés par l'HTA (75,8\%). La durée moyenne d'utilisation de la PPC par jour était de 4,7 $\pm 1,9$ heure avec un taux d'observance de 60,6\%. Les effets indésirables liés à l'utilisation de la PPC étaient présents chez $45,5 \%$ des patients et étaient dominés par l'obstruction nasale $(36,4 \%)$ et la sécheresse buccale $(21,1 \%)$. Un abandon temporaire avait été noté chez $17 / 33$ patients $(51,5 \%)$. Pour $57,6 \%$ des patients, la PPC a amélioré leur qualité de vie et pour $39,4 \%$, son effet est assez moyen sur leur état de santé.

Conclusion: De façon générale, l'utilisation de la PPC a amélioré les symptômes initialement décrits par nos patients. Toutefois, l'accessibilité financière et géographique de la PPC reste difficile dans notre contexte.

Mots clés: Syndrome d'apnées du sommeil, PPC, observance, Burkina Faso

Conflits d'intérêt: Rien à déclarer

\section{P147 \\ Etude Comparative de la Sévérité de L'infection Covid-19 Entre le Sexe Féminin et Masculin à L'hôpital Hassan 2 d'Agadir}

\author{
Aboubekr Imzil, Houda Moubachir, Hind Serhane \\ Service de pneumologie-CHR Hassan 2 d'Agadir
}

Introduction: Dès le début de la pandémie covid-19 différentes études ont relevé les formes sévères de ce virus qui s'exprimaient chez les uns plutôt que les autres, pointant notamment des différences entre jeunes et vieux, hommes et femmes. L'objectif de ce travail était de comparer la sévérité de l'infection covid-19 entre le sexe masculin et féminin à l'hôpital Hassan 2 d'Agadir.

Patients et méthode: c'est une étude rétrospective analytique et descriptive sur les dossiers de 180 patients hospitalisés au service de pneumologie de l'hôpital Hassan 2 d'Agadir suite à l'infection par le covid-19.

Résultats: la moyenne d'âge était de 60,2 ans pour le sexe masculin et 57,8 ans pour le sexe féminin. Sur le plan clinique une saturation artérielle en oxygène inférieure à $90 \%$ était retrouvée chez $6,7 \%$ des hommes versus $1,9 \%$ des femmes. Une fréquence respiratoire supérieure à 30 cycles/min était retrouvée chez 2,8\% des hommes contre $4,8 \%$ des femmes. La présence des signes de lutte respiratoire était identifiée chez 5,8\% des hommes et 1,9\% des femmes.

Biologiquement, 11,5\% des hommes ont présenté un syndrome inflammatoire face à $7,7 \%$ des femmes. Cependant le taux d'une coagulation augmentée était presque le même chez les deux sexes.

La TDM thoracique avait révélé une atteinte estimée supérieure à $50 \%$ chez $6,7 \%$ des femmes et $14,3 \%$ des hommes.

La survenue des complications thromboemboliques était rencontrée chez $5,8 \%$ des hommes versus $4,8 \%$ des femmes, $1 \%$ des hommes ont présenté une insuffisance respiratoire chronique alors qu'aucun cas n'a été signalé chez les femmes. Le taux de décès chez les hommes était de 4,8\% versus 1,9\% des femmes. 
Conclusion: A travers ce travail comparatif des facteurs de mauvais pronostic de l'infection covid-19 entre le sexe masculin et féminin, il parait que le sexe masculin est un facteur de sévérité de l'infection covid-19.

Conflits d'intérêt: Rien à déclarer

\section{P148 \\ Les Complications Thromboemboliques des Patients Covid-19 à L'hôpital Hassan 2 d'Agadir}

Aboubekr Imzil, Houda Moubachir, Hind Serhane

Service de pneumologie-CHR Hassan 2 d'Agadir

Introduction: l'infection au covid-19 était associée à la survenue des complications thromboembolique en induisant des thromboembolies dans différents organes responsables d'une aggravation clinique majeure des patients voire leur décès. L'objectif de ce travail était d'étudier les complications thromboemboliques des patients covid-19 à l'hôpital Hassan 2 d'Agadir.

Patients et méthode: c'est une étude rétrospective, analytique et descriptive sur les dossiers de 150 patients hospitalisés au service de pneumologie de l'hôpital Hassan 2 d'Agadir suite à une infection par le virus covid-19.

Résultats: la moyenne d'âge des patients était de 59 ans avec un sex-ratio de $1.18 \%$ des patients étaient des tabagiques chroniques, $43 \%$ diabétiques, 9,6\% asthmatiques, $28 \%$ des hypertendus, $3 \%$ avaient une hépatopathie chronique et $4 \%$ avaient une cardiopathie.

Le bilan biologique effectué avait montré: $16,7 \%$ des d-dimères augmentées, 0,98\% de fibrinogène augmenté, 0,98\% un taux de prothrombine diminué et aucun patient n'avait présenté de thrombopénie. 19,6\% de CRP augmentée.

Les complications thromboemboliques étaient de 9,6\%:70\% étaient des embolies pulmonaires, $20 \%$ des thromboses veineuses profondes des membres inférieurs et $10 \%$ des thromboses artérielles. $65 \%$ de ces patients étaient initialement sous traitement anticoagulant: $55 \%$ avec une dose curative, $10 \%$ avec une dose préventive; cependant $35 \%$ n'étaient pas sous traitement anticoagulants. Le taux de décès chez les patients qui avaient présenté les complications thromboemboliques était de $30 \%$.

Conclusion: l'infection par le covid-19 constitue un facteur de risque de la survenue des complications thromboemboliques d'où l'intérêt d'une évaluation minutieuse et dynamique du risque thrombotique des patients atteints de covid-19 et l'installation d'un traitement anticoagulant prophylactique efficace.

Conflits d'intérêt: Rien à déclarer

\section{P149 \\ Caractéristiques des Patients Obèses Admis Dans un Service de Pneumologie Avec Insuffisance Respiratoire Aigüe Hypercapnique: Étude Observationnelle dans une Population Nord-Africaine}

Sameh Msaad, Rahma Gargouri, Amal Saidani, Amina Kotti, Walid Feki, Jihène Trabelsi, Samy Kammoun

Service de pneumologie de l'hôpital Hedi chaker, Sfax, Tunisie

Introduction: L'insuffisance respiratoire aiguë hypercapnique (IRAH) est un événement menaçant chez les patients atteints de syndrome obésité-hypoventilation $(\mathrm{SOH})$.

Objectifs: Étudier le profil clinique des patients $\mathrm{SOH}$ admis pour IRAH, ainsi que les résultats à court et à long terme du traitement par ventilation non invasive.

Méthode: Etude de cohorte rétrospective colligeant des adultes atteints de $\mathrm{SOH} \geq 18$ ans, admis pour IRAH.

Résultats: Quarante-quatre patients ont été inclus (15 (34,1\%) avec SOH malin, $29(65,9 \%)$ avec SOH non malin, et 36 patients $(81,8 \%)$ ayant un syndrome d'apnée-hypopnée obstructive du sommeil). Trente-trois patients (75\%) étaient des femmes (âge moyen $=66,5 \pm 10,9$ ans, $\mathrm{IMC}=41,1 \pm 6,8 \mathrm{Kg} / \mathrm{m} 2)$. Un taux élevé de facteurs de risque cardiovasculaires a été noté. Les patients atteints de $\mathrm{SOH}$ malin avaient un taux significativement plus élevé d'insuffisance cardiaque ( $100 \%$ vs $31 \%$; p <0,001), d'insuffisance rénale chronique $(73,3 \%$ vs $41,4 \% ; \mathrm{p}=0,04)$ et de dyslipidémie $(66,7 \%$ vs $34,5 \% ; p=0,04)$. La capacité vitale forcée moyenne chez nos patients était de $59,5 \% \pm 18,5$ de la valeur prédite. Le taux d'échec de la BIPAP a été de 2,27\%. Quarante-deux patients ont été mis sortant sous ventilation non invasive (BIPAP ou CPAP chez 26 et 16 patients, respectivement). La survie était de $90 \%$ à 12 mois, tandis que la réadmission pour un nouvel épisode d'IRAH était de $56 \%$ à 6 mois et de $22 \%$ à 12 mois, respectivement. Aucun facteur n'était indépendamment associé à un risque plus élevé de réadmission pour IRAH.

Conclusion: L'IRAH est la circonstance de découverte le plus fréquente du $\mathrm{SOH}$. De pronostic potentiellement grave, elle se traite efficacement par la ventilation à double niveau de pression. La poursuite de la ventilation à domicile après résolution de l'épisode d'IRAH semble être associée à une faible mortalité à long terme avec un faible taux de réadmission.

Conflits d'intérêt: Rien à déclarer 


\section{P150 \\ La Pression Positive Continue Nocturne (Nppc) Réduit le Taux de la Protéine C-Réactive de Haute Sensibilité (Hs-Crp) Dans le Syndrome D'apnée- Hypopnée Obstructive du Sommeil}

Amal Saidani, Amina Kotti, Nesrine Kallel, Nadia Moussa, Rim Marrakchi, Akram Chaabouni, Sameh Msaad, Walid Feki, Samy Kammoun

Service de pneumologie de l'hôpital Hedi chaker, Sfax, Tunisie

Introduction: L'inflammation systémique et des voies respiratoires a récemment été liée au syndrome d'apnée-hypopnée obstructive du sommeil (SAHOS). Elle est aussi considérée comme un facteur de risque probable de lésions cardiovasculaires induites par le SAHOS. La hs-CRP peut être utile pour la prédiction du risque de maladie cardiovasculaire et l'évaluation de l'effet du traitement nocturne par pression-positive continue (nPPC) chez les patients SAHOS.

Méthode: Etude de cohorte prospective de 64 sujets adultes avec suspicion de troubles respiratoires du sommeil.

Résultats: Le SAHOS a été confirmé chez 43 patients (24 normotendus et 19 hypertendus) et éliminé chez 21 sujets normotendus (témoins). La moyenne de hs-CRP ne différait pas de manière significative entre les patients SAHOS et les témoins. Pareil, elle n'était associée ni à la sévérité du SAHOS $(\mathrm{p}=0,2)$, ni à l'IAH ( $\mathrm{p}=0,067)$. Un taux de hs-CRP $\geq 1 \mathrm{mg} / \mathrm{dL}$ a été plus souvent observé chez les patients atteints de SAHOS que chez les témoins $(\mathrm{p}=0,032 ; \mathrm{OR}=5,6)$ et significativement associée à l'IAH $(\mathrm{p}=0,021)$. Une diminution significative du taux médian de hs-CRP a été observée chez les patients compliant à la CPAP $(\mathrm{p}=0,006)$. Parmi ceux-ci, seuls les patients normotendus ont eu une diminution significative du taux de hs-CRP. Chez les hypertendus, la réduction du taux de hs-CRP a été non significative. En utilisant un modèle de régression linéaire, la variation du taux de hs-CRP après 6 mois de nPPC s'est avéré être positivement corrélée avec le taux de base de hs-CRP pour les SAHOS hypertendus $(\mathrm{p}=0,02 ; \mathrm{r}=0,68)$ et encore plus pour les normotendus $(\mathrm{p}<0,0001 ; \mathrm{r}=0,89)$.

Conclusion: Le traitement par nPPC peut avoir un effet cardiovasculaire protecteur chez les patients atteints de SAHOS. Le taux de hs-CRP peut servir comme outil de surveillance de l'efficacité thérapeutique de la nPPC.

Conflits d'intérêt: Rien à déclarer

\section{P151}

Apport Pronostique de FACED et BSI en Termes de Prédiction D'hospitalisation Pour Exacerbation Sévère de Bronchectasie

Haifa Zaibi, Asma Jarrar, Emna Ben Jemia, Jihen Ben Amar, Hichem Aouina

Service de pneumologie - Hôpital Charles Nicolle Tunis

Introduction: La Dilatation des bronches (DDB) est une maladie respiratoire chronique dont la sévérité est difficile à évaluer. Il s'agit d'une maladie complexe qui nécessite des outils composites pour évaluer sa sévérité. BSI et FACED sont deux scores proposé et validés ces dernières années.

Objectif: Etudier l'apport de ces scores dans la prédiction d'hospitalisation pour exacerbation de DDB.

Méthode: Il s'agissait d'une étude rétrospective analytique, colligeant les dossiers des patients atteints de dilatation des bronches suivis au service de pneumologie de l'hôpital Charles Nicolle de Tunis durant une période de 10 ans allant de 2009 à 2018.

Nous avons déterminé pour chaque malade les 5 paramètres du score FACED et les 8 items de BSI.

Résultats: Cent dix patients étaient inclus, âgés en moyenne de 57 ans, avec un sex ratio de 1,2. Le score FACED moyen était de $2,71 \pm 1,86$, classant la DDB comme légère dans $46 \%$. Le BSI moyen

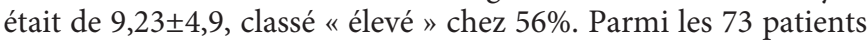
hospitalisés pour exacerbation sévère, 57 avaient un score BSI élevé, et 18 avaient un FACED sévère. L'étude de la fréquence d'hospitalisation en fonction des scores BSI et FACED, a montré une fréquence plus élevée dans le groupe sévère pour le BSI (78\% des hospitalisations $)(\mathrm{p}<0.0001)$ et dans le groupe modéré pour le FACED (47.9\% des hospitalisations) $(\mathrm{p}<0.0001)$. Concernant le risque d'hospitalisation pour exacerbation sévère, l'aire sous la courbe Roc était élevée pour les deux scores, témoignant d'une valeur discriminante élevée pour les deux. Toutefois, cette prédiction était plus importante pour le score BSI que pour le FACED $(0.95$ IC $[0,92-0,99]$ vs 0.82 IC $[0,74-0,90])$.

Conclusion: Nos résultats en concordance avec la littérature, suggèrent un rôle prédictif plus important pour le score BSI en termes d'hospitalisation pour exacerbation sévère de DDB.

Conflits d'intérêt: Rien à déclarer 
P152

\section{Apport Pronostique de Faced et Bsi en Termes de Prédiction D'oxygénothérapie au Long Court Chez les Patients Atteints de Bronchectasie}

Haifa ZAIBI, Asma JARRAR, Emna BEN JEMIA, Jihen BEN AMAR, Hichem AOUINA

Service de pneumologie - Hôpital Charles Nicolle Tunis

Introduction: Traditionnellement, le VEMS et la tomodensitométrie ont été proposés comme marqueurs de la sévérité de la DDB. Néanmoins, ces deux paramètres pris séparément manquent de corrélation. De nouveaux score (BSI et FACED) ont récemment été établi et validé pour l'évaluation de la sévérité des DDB.

Objectif: Etudier l'apport de ces scores dans la prédiction d'oxygénothérapie au long court chez les patients atteints de DDB.

Méthode: Il s'agissait d'une étude rétrospective analytique, incluant les patients atteints de DDB, suivis au service de pneumologie de l'hôpital Charles Nicolle de Tunis durant une période de 10 ans allant de 2009 à 2018.

Nous avons déterminé pour chaque malade les 5 paramètres du score FACED et les 8 items de BSI.

Résultats: Cent dix patients étaient inclus, âgés en moyenne de 57 ans, avec une légère prédominance masculine (sex ratio=1,2). Parmi les 31 patients sous OLD, 14 soit $46 \%$ avaient un score BSI élevé $(\mathrm{p}<0,0001)$, et 30 soit $97 \%$ avaient un FACED sévère $(\mathrm{p}<0.0001)$ (Tableau 31).

Également les scores moyen FACED ( 4.2 vs $2 ; \mathrm{p}<0.0001)$ et BSI ( 14 vs $7.3 ; p<0.0001$ ) étaient significativement plus élevés chez les malades sous OLD. Concernant le risque d'OLD, l'aire sous la courbe Roc était élevée pour les deux scores, témoignant d'une valeur discriminante élevée pour les deux. Cette prédiction était légèrement plus importante pour le BSI par rapport au FACED (0.89 IC [0.84-0.95] vs 0.82 IC [0.73-0.91]). L'étude de sensibilité et spécificité dans la prédiction de l'OLD, avait montré que le BSI était plus sensible que FACED à détecter les patients candidats à l'OLD (97\% vs 31\%) et qu'il avait une valeur prédictive négative meilleure ( $98 \%$ vs $81 \%)$. Cependant, BSI était moins spécifique que FACED (60\% vs 93\%) avec une valeur prédictive positive plus faible (50\% vs $73 \%)$. En considérant un seuil de 3 pour FACED, celui-ci avait une meilleure sensibilité mais qui restait toujours inférieure à celle du BSI pour prédire le recours à l'OLD $(\mathrm{Se}=83 \%$ vs $97 \%)$.

Conclusion: Il ressort de notre étude, conformément à la littérature, queleBSIsemblemieuxprédirelerecoursàl'oxygénothérapie au long court.

Conflits d'intérêt: Rien à déclarer

\section{P153 \\ Covid-19 Severity Prognosis in COPD and Asthma Patients}

Omrane Asma, Benzarti Wafa, Hajjej Sabri, Aissa Sana, Knaz Asma, Abdelghani Ahmed, Hayouni Abdelaziz, Gargouri Imen

Service de pneumologie, CHU F HACHED, Sousse, Tunisie

Introduction: Asthma and COPD (chronic obstructive pulmonary disease) are obstructive pulmonary diseases that affected millions of people all over the world. In one side Coronaviruses are among the viruses that trigger COPD and Asthma exacerbations; in the other side, Asthma and COPD have been cited as a potential risk factor for severe COVID-19. This study investigated the impact of obstructive pulmonary disorder such as asthma and COPD, on the risk for respiratory failure and mortality in COVID-19 Tunisian patients.

Materials and methods: A retrospective cohort study was conducted in 124 patients infected with COVID-19 between September 2021 and April 2021, using clinical and biological data from our pulmonology department. Asthma and COPD (chronic obstructive pulmonary disease) are defined as obstructive pulmonary diseases.

Results: The mean age was 64,73 years old and 54 patients were females. 31 were active or former smokers. Hypertension, diabetes, chronic obstructive pulmonary disease (COPD) and asthma were respectively the most common comorbidities including 31 patients with obstructive lung disease (15 patients with asthma and 16 with COPD). 112 patients required an oxygen flow higher than $10 \mathrm{~L} / \mathrm{min}$ and 16 patients required non-invasive ventilation. 22 patients were transferred to the intensive care unit (ICU), and 14 patients died in our unit. Prolonged stay in hospital (more than 2 weeks) and risk of being escalated to ICU were significantly more observed in patients with asthma and $\operatorname{COPD}(\mathrm{p}=0,05)$. A higher risk of in-hospital mortality was associated with both asthmatic patients and those having COPD ( $\mathrm{p}=0.032)$.

Conclusion: Our results suggests that people with either asthma or COPD are at higher risk for developing severe covid-19 symptoms and death. However, conflicting results have been published over the last few months and the putative association between these two diseases is still unproven.

Disclosure: Nothing to disclose

\section{P154 \\ Does Angiotensin-Converting Enzyme (ACE) Inhibitors and Angiotensin Receptor Blockers (ARB) are Protective against Covid-19 Severity?}

Omrane Asma, Hajjej Sabri, Benzarti Wafa, Aissa Sana, Knaz Asma, Abdelghani Ahmed, Hayouni Abdelaziz, Gargouri Imen

Service de pneumologie, CHU F HACHED, Sousse, Tunisie

Introduction: In observational studies, Covid-19 patients with cardiovascular comorbidities including hypertension appear to be
SSP/SSTS - EFP

Joint Annual Conference 2021 
at higher risk of mortality. Several researchers suggested that patients treated with angiotensin-converting enzyme inhibitors (ACEI) and receptor blockers (ARB) could be protected against Covid-19 severity, the aim of this study was to elucidate the correlation between angiotensin-converting enzyme inhibitors (ACEI)/ receptor blockers (ARB) and COVID-19 infection.

Materials and methods: Patients admitted to our pulmonology department from September 2020 to April 2021, with positive RT-PCR results for COVID-19, were included in this study. Clinical and biological data of 124 patients were prospectively investigated and compared.

Results: The mean age was 64,73 years old and 54 patients were females. 31 were active or former smokers. Hypertension, diabetes, chronic obstructive pulmonary disease (COPD) and asthma were respectively the most common comorbidities. 41 patients were on long-term therapy with ACE inhibitors or ARBs. 114 patients required an oxygen flow higher than $10 \mathrm{~L} / \mathrm{min}$ and 16 patients required non-invasive ventilation. 22 patients were transferred to the intensive care unit (ICU), and 14 patients died in our unit. Multivariate analysis showed a significant correlation between ACE inhibitors or ARBs and mortality of COVID-19 infection $(\mathrm{p}=0,038)$.

Conclusion: Our preliminary results suggested a significant correlation between ACE inhibitors or ARBs and COVID-19 infection severity. These results have to be confirmed, and do not question the current recommendations to continue this long-term treatments.

Disclosure: Nothing to disclose

\section{P155}

\section{Particularités de la Tuberculose Chez les Tabagiques}

Zaâfouri Asma, Moussa Ines, Sahnoun Imen, Ben sassi Sarra, dabebbi Fatma zahra, Maâlej Sonia, El Gharbi Douik Leila

pavillon D - hôpital Abderahmen Mami I'Ariana

Introduction: Le tabagisme et la tuberculose sont deux enjeux majeurs de santé publique au niveau mondial. L'évaluation de l'influence du tabagisme sur la tuberculose est d'une importance capitale.

Le but de notre travail est d'étudier les conséquences du tabagisme sur les manifestations cliniques, radiologiques et évolutives de la tuberculose pulmonaire.

Méthode: Il s'agissait d'une étude rétrospective comparative menée entre Janvier 2015 et décembre 2020 dans notre service de pneumologie Pavillon D de l'hôpital de Aberrahmen Mami de l'Ariana, incluant 90 patients ayant une tuberculose pulmonaire ou extra pulmonaire répartis en deux groupes: Groupe 1 (G1) les tabagiques: 31 patients et Groupe 2 (G2): les non tabagiques: 59 patients.

Résultats: L'âge moyen dans le groupe des tabagiques était de 42,5 ans contre 46,2 ans dans G2. Le degré du tabagisme était estimé à 22,8 PA. Il n'y avait pas une différence significative entre le délai de la consultation entre les deux groupes. Les symptomatologies étaient non spécifiques dans les 2 groupes et dominés par la toux $(67 \%$ vs $55 \%)$ sans différence significative. Sur le plan radiologique, il y avait des lésions excavées dans 37\% des cas dans le groupe des fumeurs contre $62 \%$ dans le groupe des non-fumeurs. Le retard de négativation des bascilloscpies était plus important chez $\mathrm{G} 1$ avec $\mathrm{p}=0,008$. Les séquelles radiologiques étaient plus fréquentes dans le groupe des fumeurs $(\mathrm{p}=0,04)$.

Conclusion: L'association tuberculose et tabac ne modifie pas l'aspect clinique et radiologique de la tuberculose mais conditionne son évolution et expose plus au risque d'avoir des séquelles radiologiques. Le sevrage tabagique doit faire partie intégrante de la prise en charge du patient atteint de tuberculose.

Conflits d'intérêt: Rien à déclarer

\section{P156 \\ Cancer Broncho Génique Primitif Chez la Femme Particularités Clinique, Histologique et Évolutives A Propos de 17 Cas}

\author{
Btissame Es-sabbahi, Siham Iqraouin, Bouchra Amara, \\ Mohammed Elbiaze, Mohammed Chakib Benjelloun, \\ Mounia Serraj
}

Service de pneumologie, CHU Hassan II Fès

Introduction: Le profil classique du patient porteur d'un cancer du poumon est en train d'évoluer, passant de l'homme âgé tabagique à la femme plus jeune et volontiers non fumeuse. Le cancer bronchique chez la femme a de nombreuses spécificités aussi bien pour les facteurs de risques, la présentation clinique et la prise en charge diagnostique et thérapeutique.

Matériel et méthode: C'est une étude observationnelle rétrospective, portant sur 17 cas de cancers bronchique primitif, diagnostiqué au service de pneumologie du CHU Hassan II Fès, entre Mars 2019 et Février 2021.

Résultats: L'âge moyen de nos patientes était de 65,12 ans (44 à 83 ans), $11.8 \%$ des patientes était exposées au tabagisme passif, 2 patientes étaient tabagiques actives (11.8\%). Les circonstances de découvertes sont principalement la toux associée à une dyspnée chez 7 patientes soit $41.2 \%$, la toux isolée trouvée chez 4 patientes soit $23.5 \%$, l'hémoptysie chez 3 patientes (17.6\%), la dysphonie chez une patiente (5.9\%), la dyspnée chez une patiente soit $5.9 \%$, et la douleur thoracique type pleural chez une patiente (5.9\%).

La fibroscopie bronchique réalisée chez toutes les patientes, un bourgeon a été trouvé chez 5 patientes (29.4\%), Sténose infiltrative chez 7 patientes $(41,2 \%)$, et l'aspect endoscopique était normal chez 5 patientes (29,4\%). Le diagnostic histologique a été obtenu par biopsies bronchiques chez 8 patientes (47.1\%), biopsie guidées par le scanner chez 8 patientes (47.1\%), guidées par l'échographie chez une patiente $(5.9 \%)$ et par biopsie pleural chez une patiente (5.9\%). L'adénocarcinome représente le type histologique le plus fréquent avec 15 cas $(88.2 \%)$, la recherche du profil mutationnel a été faite chez toutes nos patientes. 15 patientes soit $88.2 \%$ étaient métastatiques.

Conclusion: Une meilleure compréhension des particularité cliniques, anatomopathologique, biologique, et évolutive des cancers bronchiques chez la femme permettra d'ouvrir de nouvelles 
voies de recherche et de développer des prises en charge plus personnalisées afin d'améliorer le pronostic des patients atteints d'un CBNPC. Ceci nécessite la réalisation d'enquêtes analytiques menées idéalement de façon multicentrique.

Conflits d'intérêt: Rien à déclarer

\section{P157 \\ Les Facteurs Prédictifs de Gravité Clinique, Biologique et Radiologique Chez les Patients Atteints du COVID-19}

\section{Rachid, S. Ait batahar, L. Amro}

Pneumologie, Hopital Arrazi, Chu mohamed VI Marrakech

Introduction: La présentation clinique de l'infection à virus SARS-Cov-2 est très variable, allant des formes bénignes aux formes graves marquées notamment par un syndrome de détresse respiratoire aigue.

But: Déterminer les facteurs prédictifs de gravité clinique, biologique et radiologique chez les patients ayant une infection SARS-Cov-2.

Méthode: Une étude transversale descriptive et analytique menée auprès des patients hospitalisés pour la COVID-19 durant la période de octobre 2020 à Décembre 2020 en unité de soins intensifs CHU de Marrakech confirmée et présentant une détresse respiratoire aiguë. Les variables retenues dans le modèle final ont été sélectionnées en utilisant une méthode stepwise forward avec un seuil d'entré à 0,2 et un seuil de sortie à 0,05 . Le seuil de significativité était retenu pour un $\mathrm{p}<0,05$.

Résultats: 125 cas de COVID-19 ont été colligés (40,8 femmes et 59,2 hommes). La moyenne d'âge était de 59,6 ans. 86,4\% étaient exposé au tabac. $66,7 \%$ étaient fumeur actif et $33,3 \%$ étaient fumeur passif. La présence de comorbidités était notée dans $77,6 \%$ des cas. Il s'agissait de l'hypertension artérielle dans $(28,4 \%)$ des cas, du diabète $(32,6 \%)$, de cardiopathies $(7,1 \%)$, de néoplasies $(5,7 \%)$, de dysthyroïdies $(5,7 \%)$ et d'asthme bronchique (6,4\%). Les examens de confirmation comportaient dans $76,9 \%$ une imagerie évocatrice, $47,9 \%$ une pcr positive. Les symptômes respiratoires prédominant étaient la toux dans $84 \%$ des cas, suivi de la dyspnée dans $70,9 \%$ des cas. Les signes extra-respiratoires prédominant étaient l'asthénie dans $79 \%$ des cas, suivie par les myalgies dans $46 \%$ des cas, l'anosmie $29,4 \%$ des cas et l'agueusie dans $21 \%$ des cas. La fièvre était retrouvé chez $68 \%$ des patients. 35\% étaient tachycarde à $>90 \mathrm{bat} / \mathrm{min}$ et $43,2 \%$ des patients avaient une saturation $<90 \mathrm{bat} / \mathrm{min}, 16 \%$ une sa02 entre $90-95 \%$. 71 patients ayant une saturation $>90 \%$ avaient une moyenne d'âge de 57,3 ans alors que 54 patients avec une sao $2<90 \%$ avaient une moyenne d'âge de 62,7 avec un ( $\mathrm{p}=0,027) .42$ patients ayant une saturation $<90 \%$ avaient un taux de lymphocytes moyen à 1123 avec un ( $\mathrm{p}=0,003)$. La TDM thoracique a révélé un aspect de verre dépoli chez $83,2 \%$, des condensations alvéolaires chez $40 \%$. Les lésions étaient critiques dans $12,8 \%$ des cas, sévères dans $30,8 \%$ des cas, modérées dans $30,8 \%$ des cas, et légères dans $25,6 \%$ des cas. Des images radiologiques en faveur de surinfections pulmonaires ont été notées dans $77 \%$ des cas. L'extension radiologique a été corrélée à la saturation: $76,1 \%$ des patients avec une sa02 $>90 \%$ avaient une extension pulmonaire légère à modéré avec un $(\mathrm{p}=0,0001)$. Des perturbations biologiques ont été constatées également. Il s'agissait d'un taux élevé des $\mathrm{D}$ dimères dans $(31 \%)$ des cas, d'une lymphopénie $(47,4 \%)$, d'une leucopénie (8\%), d'une thrombopénie (13\%), d'une hyperleucocytose à PNN (34,2\%), d'une cytolyse hépatique (10\%), d'un taux de CRP élevé (54\%), d'un taux de fibrinogène élevé (59\%), et d'une fonction rénale perturbée (36\%). La corrélation entre taux de lymphocytes initiale et l'extension radiologique était comme suit: 31 patients avec une extension radiologique sévère et un taux de lymphocytes moyen à $1201(p=0,005)$. La corrélation entre le taux de CRP initiale et l'extension radiologique: 35 patients ayant une extension sévère avec une crp moyenne à 157 $(\mathrm{p}=0,001)$. L'évolution était marquée par l'admission en unité de soins intensifs de la totalité des patients avec mise en place d'une assistance ventilatoire chez $91 \%$ des patients. $84 \%$ des patients avaient un débit d'O $2<10 \mathrm{l} / \mathrm{min}$ avec une extension pulmonaire légère à modérée avec un $(\mathrm{p}=0,011)$. Un seul cas de décès a été constaté.

Conclusion: L'évolution des patients atteints de COVID-19 vers l'aggravation semble être associée à l'âge, aux antécédents cardiovasculaires et métaboliques, à la sévérité de l'atteinte radiologique, et aux perturbations biologiques observées.

Conflits d'intérêt: Rien à déclarer

\section{P158 \\ Covid-19: Du Diagnostic au Pronostic}

\section{Rachid, S. Ait batahar, L. Amro}

Pneumologie, Hopital Arrazi, Chu mohamed VI Marrakech

Introduction: La propagation du coronavirus SARS-CoV-2, découvert en Chine en janvier 2020, a mené à une pandémie dès mars 2020, obligeant chaque système de soins des pays touchés à une adaptation rapide. Pour mieux faire face à cette crise sanitaire majeure, qui a donné' lieu à de nombreuses publications scientifiques, nous avons évaluer les caractéristiques cliniques et le pronostic du Covid-19 en USI de l'hôpital Mohammaed VI.

But: Savoir si la gravité de la maladie et le type de prise en charge conditionnaient le pronostic du patient en terme de complications et de faculté de récupération.

Méthode: L'analyse descriptive a consisté au calcul des fréquences absolues et relatives pour les variables qualitatives, et des paramètres de positionnement et de dispersion pour les variables quantitatives (moyenne, écart-type). Pour la comparaison de pourcentages en analyse bivariée, le test statistique du Chi2 de Pearson et celui de Fisher si nécessaire ont été utilisés. L'analyse multivariée a été réalisée par la régression logistique.

Elle convient aux modèles dans lesquelles les variables sont dichotomiques (bon/ mauvais pronostic). Ainsi, les variables dont l'association était significative au seuil de $20 \%$ en analyse bivariée ont été incluses dans un modèle multivarié. Le seuil de significativité était retenu pour un $\mathrm{p}<0,05$.

Résultats: La cohorte constituée entre octobre et décembre 2020 comportait 125 patients avec un Covid-19, classés en deux 
groupes selon la sévérité. L'âge médian des patients participants était de 59,6 ans. 73,6\% (92) des patients ont quitté l'USI après avoir achevé le traitement, $16,8 \%$ (21) ont été transféré en unité de réanimation, $8,8 \%$ (11) dans une unité d'hospitalisation et $0,8 \%$ (1) sont décédés. $73,6 \%$ (92) sont considérés comme ayant un bon pronostic et $26,4 \%$ (33) un mauvais pronostic. Les facteurs ayant conditionnés le pronostic sont: l'âge, le taux de lymphocytes, les D-dimères, la fréquence respiratoire, la saturation et le débit d'o2. La corrélation entre l'âge et l'évolution pronostic: 92 patients avec un âge moyen de 57,9 ans +/- 13,03 avaient un bon pronostic contre 33 patients avec un âge moyen de 64,52 ans +/- 13,97 avaient un mauvais pronostic avec un $(\mathrm{p}=0,016)$. Le taux de lymphocytes était également un facteur qui a conditionné le pronostic: 44 patients avec un taux de lymphocytes moyen à 1514 étaient de bon pronostic contre 21 patients de mauvais pronostic avec un taux de lymphocytes moyen à 1120 avec un $(\mathrm{p}=0,037)$. Le dosage des D-dimères: 37 patients avec un taux de d-dimères moyen à 1265 avaient un bon pronostic contre 18 patients avec un taux de $\mathrm{D}$-dimères moyen à 9193 avec un $(\mathrm{p}=0,023)$. La fréquence respiratoire: chez les patients ayant une fréquence respiratoire $<24$ cycles/ min: $85,7 \%$ (48) d'entre eux avaient un bon pronostic, $14,3 \%(8)$ un mauvais pronostic avec un $(p=0,006)$. La saturation en 02 : $83,1 \%$ (59) des patients ayant une saturation $>90 \%$ ont bien évolués comparés à $61,1 \%$ (33) des patients avec une saturation $<90 \%$ avec un $(\mathrm{p}=0,08) .86,8 \%$ des patients ayant un bon pronostic avaient un débit d'02 <10L/min alors que $65,5 \%$ avaient un mauvais pronostic avec un débit d'02 $>10 \mathrm{~L} / \mathrm{min}$ avec un $(\mathrm{p}=0,0001)$. La sortie avec un extracteur d'oxygène était considéré comme un élément de mauvais pronostic soit chez $71 \%$ des patients avec un $(\mathrm{p}=0,0001)$.

Conclusion: Cette étude montre doncl'importance des facteurs prédictifs notamment l'âge, le taux de lymphocytes et les d-dimères, la fréquence respiratoire et la saturation en o2 en tant que facteur pronostique de l'évolution de l'infection à Covid-19 avec une aggravation rapide synonyme de sévérité de l'évolution.

Conflits d'intérêt: Rien à déclarer

\section{P159 \\ La Prévalence des Troubles du Sommeil Chez les Étudiants en Médecine d'Agadir}

\section{Fatiiha. Bounoua, Abdelkrim. Khannous, Houda. Moubachir, Hind. Serhane}

Service de pneumologie hôpital Hassan d'Il d'Agadir, Maroc

Introduction: Les troubles du sommeil chez les étudiants sont fréquents et préoccupants compte tenu des conséquences multiples qu'ils entraînent sur la santé mentale et physique ainsi que sur le rendement scolaire.

L'objectif de cette enquête est d'évaluer la prévalence, les facteurs déterminants ainsi que le retentissement des troubles du sommeil chez les étudiants en médecine d'Agadir.

Matériel et méthode: Nous avons mené en octobre 2020 une étude transversale descriptive auprès de 136 étudiants de médecine d'Agadir, par l'administration d'un questionnaire inspiré du questionnaire des troubles de sommeil de l'hôtel-Dieu (QSHD), envoyé par e-mail aux étudiants de la première à la 7eme année.
Résultats préliminaires: La moyenne d'âge était de 20,98 ans, avec prédominance féminine (75\%).

La moyenne de temps de sommeil était de $8 \mathrm{~h}$ au cours de la semaine et de $9 \mathrm{H}$ le week-end. La sieste quotidienne était notée auprès de 68 (50\%) étudiants, on a constaté qu'une sieste de plus de 20 minutes pourvoyait plus à la fatigue au réveil.

La prévalence des symptômes d'insomnie était de 60,22\%, ils étaient associés à l'usage d'excitants, de l'internet en soirée, l'usage d'hypnotiques ne concernait que $22,5 \%$ des insomniaques; nous avons noté une prévalence élevée des cauchemars avec 47\%, $17,64 \%$ de bruxisme et $5 \%$ de la paralysie du sommeil tous les trois fortement associés au stress.

La prévalence de la Somnolence diurne excessive selon l'échelle d'Epworth était de 22,1\%, le ronflement était présent chez 4 étudiants $(2,94 \%)$.

58,08 des étudiants trouvaient des difficultés de concentration et $36,02 \%$ estimaient que leurs rendement n'est pas conforme aux attentes.

Conclusion: La forte prévalence des troubles du sommeil et des facteurs associés chez cette catégorie de population doit alerter tous les professionnels de santé afin de les dépister précocement et de mener des actions de prévention.

Conflits d'intérêt: Rien à déclarer

\section{P160 \\ Attitude du Personnel de la Santé de L'hôpital Hassan li d'Agadir (Maroc) Vis à Vis le Vaccin Contre la COVID-19}

\author{
Fatiiha. Bounoua, Abdelkrim. Khannous, Houda. Moubachir, \\ Hind. Serhane
}

Service de pneumologie hôpital Hassan d'Il d'Agadir, Maroc

Introduction: La COVID-19 est une maladie grave. Le virus SARS-CoV-2 a infecté plus de 130 millions de personnes dans le monde, entraînant 2,91 millions de décès, les vaccins offrent une meilleure voie de sortie de la pandémie de COVID-19 et les scientifiques ont maintenant mis au point plusieurs vaccins très efficaces contre la COVID-19.

Le but de ce travail est d'évaluer l'attitude du personnel de la santé de l'hôpital Hassan II d'Agadir (Maroc) vis à vis le vaccin contre la COVID-19.

Matériel et méthode: Il s'agit d'une étude transversale exhaustive à visée descriptive et analytique réalisée auprès de personnel exerçant à l'hôpital Hassan II d'Agadir. La collecte des données a eu recours à la construction d'un web questionnaire sur la plateforme Google Forms.

Résultats préliminaires: Parmi les 93 participants, 58,21\% étaient des femmes, la moyenne d'âge était de $33,3 \pm 5$ ans. $32 \%$ des patients avaient des comoridités, 38, 34\% ont fait la COVID-19. Le taux de vaccination était de $82,98 \%$ dont les causes étaient dominés par: le contrôle de l'épidémie dans $98,45 \%$, pour se protéger $(91,34 \%)$ et pour ne pas avoir des problèmes administratifs dans l' avenir en cas de demande d'attestation de vaccination (67,33\%). 19,52\% n'ont pas l'attention d'être vacciné, les raisons 
étaient: L'incertitude sur l'efficacité du vaccin(86,89\%), pour ne être parmi les 1 ères vaccinés (74\%)et la crainte d'effets secondaires à long terme $(69,12 \%)$.

$56,94 \%$ des participants n'étaient pas optimiste sur le rôle du vaccin pour la lutte contre cette pandémie.

Conclusion: Le taux de vaccination chez le personnel de santé reste modeste. Un réel travail de sensibilisation et d'information sur la vaccination et les moyens de prévention est nécessaire pour la protection des patients et du personnel de santé.

Conflits d'intérêt: Rien à déclarer

\section{P161 \\ Le Cadre Nosologique de L'embolie Pulmonaire au Service de Pneumologie de L'hôpital Hassan li d'Agadir (Maroc)}

\section{Fatiiha. Bounoua, Houda. Moubachir, Hind. Serhane}

Service de pneumologie hôpital Hassan d'Il d'Agadir, Maroc

Introduction: L'embolie pulmonaire fibrino-cruorique: se définit comme l'oblitération totale ou partielle du tronc ou d'une branche de l'artère pulmonaire par un embole provenant le plus souvent d'une thrombose veineuse profonde des membres inférieurs. L'objectif du travail a été d'étudier les aspects cliniques et évolutifs de l'embolie pulmonaire fibrino-cruorique à l'hôpital Hassan II d'Agadir.

Matériel et méthode: C'est une étude rétrospective, analytique et descriptive se basant sur les dossiers des patients hospitalisés pour embolie pulmonaire confirmé par l'angioscanner thoracique au service de pneumologie du CHR HASSAN II d'AGADIR.

Résultats: Le sex ratio femme/homme a été de deux. la moyenne d'âge des patients a été de 53,57 ans. Les facteurs de risque de maladies thrombo -embolique veineuse ont été dominés par: le traitement contraceptif oral (50\%), l'alitement (46,66\%), l'insuffisance respiratoire chronique $(33,3 \%)$ et les antécédents de chirurgie récente $(26,66 \%)$. Les principaux symptômes ont été: dyspnée $(93,33 \%)$, douleur thoracique $(73,33 \%)$, toux (60\%) et l'hémoptysie (26,66\%). 6,66\% des cas ont été en état de choc cardiogénique. La probabilité clinique selon le score de Genève a été élevé dans $56,66 \%$. L'ECG a noté: une tachycardie sinusale dans (60\%), La radiographie thoracique a noté une anomalie de AMG dans $(53,33 \%)$, cardiomégalie dans (30\%), L'angioscanner thoracique a été anormal dans $100 \%$ des cas. Le traitement a été fait de l'héparine de bas poids moléculaire avec un anti vitamine $\mathrm{k}$ dans $66,6 \%$. Une thrombolyse a été effectuée chez huit malades. L'évolution a été favorable dans $80 \%$ des cas. Le taux de létalité a été de $6.66 \%$.

Conclusion: L'embolie pulmonaire pose un problème de prise en charge à cause du coût élevé des examens complémentaires et du traitement. La prévention reste donc l'arme efficace.

Conflits d'intérêt: Rien à déclarer

\section{P162 \\ Le Profil Radiologique des Patients Porteurs de Tuberculose Pulmonaire au Centre de Diagnostic de Tuberculose et de Maladie Respiratoire d'Agadir}

Fatiiha. Bounoua, Abdelkrim. Khannous, Houda. Moubachir, Hind. Serhane

Service de pneumologie hôpital Hassan d'Il d'Agadir, Maroc

Introduction: La tuberculose (TBK) est la 9ème cause de décès dans le monde. Dans notre pays elle continue à sévir à l'état endémique où l’on dénombre chaque année presque 26000 nouveaux cas. Les signes radiologiques de la tuberculose pulmonaire sont très variables mais dans la plupart des cas, ils sont suffisamment caractéristiques pour suggérer le diagnostic.

Le but de notre travail est de déterminer le profil radiologique des patients porteurs de tuberculose pulmonaire au centre de diagnostic de tuberculose et de maladie respiratoire d'Agadir.

Méthodes: étude rétrospective des dossiers des patients ayant une tuberculose pulmonaire colligés au centre de diagnostic de tuberculose et de maladie respiratoire d'Agadir entre janvier 2019 et mars 2020 .

Résultats: Il s'agit de 157 patients, la moyenne d'âge était de $39,15+/-2,38$ ans. Une prédominance masculine a été notée dans $61,8 \%$ avec un sex-ratio de 1,61. 2,54\% des patients étaient déjà traités pour TPM+. La notion de contage tuberculeux a été notée dans 4,45\%. Aucun patient n'a présenté une co-infection HIV-TBK. Les signes fonctionnels étaient prédominés par la toux etl'altération de l'état général a été trouvée dans respectivement $89,8 \%$ et $92,35 \%$ des cas. Les lésions radiologiques étaient bilatérales dans 51,59\% des cas, occupant le tiers supérieur dans $63,05 \%$ avec prédominance des infiltrats dans 56,05\% des cas. Les lésions excavées ont été retrouvées dans $45,27 \%$ des cas, des opacités nodulaires et micronodulaires dans $7,64 \%$, des opacités d'allure alvéolaire dans $12,73 \%$ des cas. Des images radiologiques associées évocatrices de BPCO étaient notées dans 6,36\% et d'épanchement liquidien dans $5,09 \%$. L'existence de plusieurs lésions radiologique chez le même patient est aussi possible.

Conclusion: Malgré que les aspects radiologiques de la tuberculose pulmonaire soient souvent évocateurs, elle reste une préoccupation quant à sa prise en charge diagnostique et thérapeutique

Conflits d'intérêt: Rien à déclarer 


\section{P163 \\ Prise en Charge des Fistules Bilio-Thoraciques: 20 Ans D'expérience Dans un Seul Centre}

\section{Faycal El Oueriachi, Mohamed Bhairis, Omar Slaoui,} Mouad Amraoui, El Hassane Kabiri

Chirurgie Thoracique, Hopital militaire d'instruction Mohamed $\mathrm{V}$ de RABAT

Introduction: Les fistules bilio-thoraciques (FBT), complication connue redoutable des kystes hydatiques du foie (KHF) négligés, demeurent exceptionnelles.

Méthode: De Janvier 2001 à Décembre 2020, une étude rétrospective colligeant 24 patients opérés dans notre institution pour fistule bilio-thoracique a été effectuée. Les particularités épidémiologiques, cliniques, anatomiques, évolutives et thérapeutiques de nos patients ont été relevées et analysées.

Résultats: L'âge moyen des patients était de 48,2 ans, 6 malades connus porteurs de KHF intacts non traités, 10 autres déjà opérés pour KHF, pour les 8 patients restants l'apparition des complications thoraciques était inaugurale. Tous les malades étaient symptomatiques. La tomodensitométrie thoraco-abdominale avait permis de confirmer le diagnostic de FBT, de dresser un bilan lésionnel précis et de planifier le geste chirurgical dans tous les cas. Une sphinctérotomie endoscopique préopératoire a été effectué chez 8 patients. La voie d'abord était une thoracotomie dans 22 cas et une thoraco-phréno-laparotomie dans 2 cas. La pneumonectomie était effectuée dans 1 cas, la lobectomie inférieure ou moyenne dans 8 cas, la bilobectomie dans 1 cas, la phrénoplastie était possible chez tous les malades, le traitement des fistules biliaires dans 14 cas et la décortication dans 18 cas. Selon la classification de Mestiri: 11 cas classés type 1, 4 cas type 2, 6 cas type 3 et 3 cas type 4. La mortalité opératoire était de 20,8\%. La durée moyenne d'hospitalisation était de 28 jours dont 5 jours en unité de soins intensifs. Le suivi post opératoire est compris entre 6 et 228 mois.

Conclusion: Quoique l'incidence des FBT ait nettement régressé à cause d'une meilleure prévention et d'une prise en charge précoce et optimisée de la maladie hydatique, certains cas demeurent d'actualité et revêtent parfois des formes particulières.

Conflits d'intérêt: Rien à déclarer

\section{P164}

\section{Embolie Pulmonaire en Post Covid-19}

\section{F. Ahsino, A. Zegmout, H. Souhi, H. Elouazzani, I. Rhorfi, A. Abid}

Service de pneumologie, Hôpital militaire d'instruction Mohamed V, Rabat, Maroc

Introduction: L'infection Covid-19 s'accompagne d'un risque thrombotique élevé due à l'hypercoagulabilité qui associe l'infection virale entraînent un dysfonctionnement des cellules endothéliales et donc des effets microangiopathiques. Le but de notre travail est de décrire les caractéristiques cliniques et radiologiques de l'embolie pulmonaire (EP) chez les patients déjà traités pour Covid-19.

Méthode: Il s'agit d'une étude rétrospective portant sur 5 patients traités pour Covid-19, avec un test PCR de contrôle négatif, hospitalisés au service de pneumologie de l'Hôpital Militaire d'Instruction Mohamed V de Rabat pour une EP confirmée par l'angioscanner thoracique.

Résultats: L'âge moyen était 61,4 ans (40-93 ans), tous de sexe masculin. 1 patient était diabétique et 1 hypertendu. Le diagnostic de Covid-19 a été retenu sur les images scannographiques et le test PCR qui était positif. Tous nos patients ont été traités par l'azithromycine et la vitaminothérapie, $60 \%$ (3 patients) ont pris le plaquénil et qu'un seul patient qui a reçu un traitement anticoagulant prophylactique. La durée moyenne entre le diagnostic de Covid-19 et les signes cliniques de l'EP était 17 jours (15-20 jours). $80 \%$ de nos patients ont présenté une dyspnée, $40 \%$ douleur thoracique. L'examen clinique ne trouvait pas des signes de retentissement cardiaque droit. Un angioscanner thoracique a été fait chez tous nos patients qui a objectivé une EP proximale droite chez 3 patients, une EP distale droite chez 1 patient et une EP bilatérale chez 1 patient. 4 patients ont été mis sous HBPM en chevauchement avec les antivitamines $K$ et 1 patient a été mis sous les anticoagulants oraux directs (AOD). L'évolution clinique était favorable chez tous nos patients.

Conclusion: A travers notre étude, nous insistons sur la place du traitement anticoagulant prophylactique chez tous les patients Covid-19 et de penser au diagnostic de l'EP en post Covid-19 devant l'apparition ou aggravation des signes respiratoires.

Conflits d'intérêt: Rien à déclarer

\section{P165 \\ Profil Radio-Clinique et Thérapeutique du Pneumothorax: à Propos de 157 Cas}

F. Ahsino, A. Zegmout, H. Souhi, H. Elouazzani, I. Rhorfi, A. Abid

Service de pneumologie, Hôpital militaire d'instruction

Mohamed V, Rabat, Maroc

Introduction: Le pneumothorax est défini par la présence de l'air dans la cavité pleurale. Les étiologies sont diverses. Il nécessite une prise en charge rapide et adéquate.

Le but de notre travail est d'étudier les différents aspects cliniques et radiologiques du pneumothorax ainsi que la prise en charge thérapeutique.

Méthode: Une étude rétrospective portant sur 157 pneumothorax hospitalisés au service de pneumologie de l'Hôpital Militaire d'Instruction Mohammed V de Rabat sur 6 ans (entre Janvier 2015 et Décembre 2020).

Résultats: Nous avons colligés 150 hommes et 7 femmes. L'âge moyen était de 45,62 ans. 86 patients étaient tabagiques $(54,8 \%)$. L'antécédent de tuberculose pulmonaire était retrouvé chez 28 patients $(17,9 \%)$, le néo pulmonaire chez 5 patients $(3,2 \%)$. Le pneumothorax était récidivant chez 26 patients (16,6\%). La symptomatologie clinique était dominée par la douleur thoracique et la dyspnée. La radiographie thoracique objectivait un pneumothorax 
unilatéral total chez 116 patients(73,9\%), unilatéral partiel chez 23 patients (14,7\%), bilatéral chez 2 patients et un hydro-pneumothorax chez 16 patients(10,2\%). Le côté droit était le plus touché(61\%). Les principales étiologies étaient: un emphysème bulleux chez 30 patients(19,1\%), les séquelles de tuberculose chez 28 patients(17,9\%), la néoplasie chez 5 patients(3,2\%), un asthme chez 3 patients $(2 \%)$ et une pneumopathie infiltrative diffuse chez un patient. 5 cas de pneumothorax iatrogène étaient provoqués par la biopsie scannoguidée( 3 cas) et la biopsie pleurale( 2 cas). Le traitement était basé sur l'abstention thérapeutique chez 18 patients(11,5\%), l'exsufflation seule chez 8 patients(5,1\%), le drainagethoraciquesousaspiration doucechez 115 patients $(73,2 \%)$. La chirurgie était indiquée chez 52 patients(33\%) pour: la persistance du bullage( 24 patients), un pneumothorax récidivant(26 patients) et un pneumothorax bilatéral(2 patients). L'évolution était favorable chez l'ensemble de nos patients.

Conclusion: A travers notre étude, nous constatons que le pneumothorax est plus fréquent chez l'homme et le drainage thoracique reste la méthode la plus utilisée dans notre service.

Conflits d'intérêt: Rien à déclarer

\section{P166 \\ Tuberculose Pulmonaire Pseudo-Tumorale}

F. Ahsino, A. Zegmout, H. Souhi, H. Elouazzani, I. Rhorfi, A. Abid

Service de pneumologie, Hôpital militaire d'instruction Mohamed V, Rabat, Maroc

Introduction: La forme pseudo tumorale de la tuberculose pulmonaire est rare. Elle peut simuler un cancer broncho-pulmonaire par sa présentation clinique, radiologique et endoscopique.

Le but de notre travail est de souligner les difficultés diagnostiques de ces formes de tuberculose.

Méthode: Nous rapportons 10 cas de tuberculose pulmonaire pseudo-tumorale colligés au service pneumologie de l'Hôpital Militaire d'Instruction Mohamed V de Rabat sur 2 ans entre Janvier 2019 et Décembre 2020.

Résultats: L'âge moyen était de 44,4 ans (23 - 67 ans), tous de sexe masculin. Le tabagisme était retrouvé chez 6 patients. Le tableau clinique est dominé par la douleur thoracique dans $60 \%$ des cas, la dyspnée et la toux dans $30 \%$ des cas chacune et l'hémoptysie dans $20 \%$ des cas. L'altération de l'état général est notée chez $40 \%$ des patients. La radiographie thoracique montre une opacité apicale dans $60 \%$ des cas, une opacité à projection hilaire dans 30\% des cas et basale dans $10 \%$ des cas. Le scanner thoracique avait objectivé une condensation parenchymateuse dans 6 cas et une masse tissulaire d'allure tumorale dans 4 cas. La fibroscopie bronchique était normale chez 4 cas. Elle avait objectivé un bourgeon endo-bronchique et un aspect inflammatoire chez 3 cas chacun. 2 patients ont bénéficié d'une biopsie transpariétale. Le diagnostic de tuberculose pulmonaire était confirmé par la bactériologie dans 6 cas, la biopsie bronchique dans 3 cas et la biopsie trans-pariétale dans 1 cas. L'évolution sous traitement anti bacillaire était favorable dans environ $90 \%$ des cas.
Conclusion: La tuberculose pulmonaire pseudo-tumorale, malgré sa présentation atypique et trompeuse, doit être évoquée devant toute atteinte pulmonaire même d'allure tumorale surtout dans un contexte épidémiologique et clinique évocateur.

Conflits d'intérêt: Rien à déclarer

\section{P167 \\ Contrôle de L'asthme Chez les Patients Atteints du Syndrome de Chevauchement Asthme/ Syndrome d'Apnée Obstructive du Sommeil en Tunisie}

\section{Maroua Bougacha, Ferdaous Yangui, Héla Cherif, Mariem Triki, Bochra Karaborni, Mohamed Ridha Charfi}

Service de pneumologie. Hôpital des FSI. La Marsa.

Tunis. Tunisie

Introduction: L'asthme et le syndrome d'apnée obstructive du sommeil (SAOS) partagent plusieurs facteurs de risque et comorbidités tel que l'obésité. Le syndrome de chevauchement asthme et SAOS présente des caractéristiques cliniques particulières. En effet, le SAOS est impliquée dans le mauvais contrôle de l'asthme. L'objectif de cette étude est d'évaluer le contrôle de l'asthme chez les patients présentant un syndrome de chevauchement « asthme et SAOS ».

Méthode: Il s'agissait d'une étude descriptive rétrospective réalisée de 2019 à 2021 et incluant des patients suivis pour asthme et SAOS. Le SAOS était confirmé par une polygraphie ventilatoire avec un indice d'apnée hypopnée $\geq 5 / \mathrm{h}$. Le contrôle de l'asthme a été évalué selon les recommandations du GINA. Les patients ont été divisés en 2 groupes; G1: 59 patients avec un asthme contrôlé et G2: 48 patients avec un asthme partiellement ou non contrôlé.

Résultat: Un total de 107 patients a été inclus avec un âge moyen de 57 ans et une prédominance féminine $(67.29 \%$ de femmes). L'IMC moyen était de $35,5 \mathrm{~kg} / \mathrm{m} 2$ et $79,5 \%$ des patients étaient obèses. L'asthme était allergique chez 30,8\% des patients. L'IAH moyen était de 28,9/h. Il n'y avait pas de différence entre les deux groupes en ce qui concerne l'âge ( 56 vs 58, 3 ans), l'IMC ( 35 vs $36,1 \mathrm{~kg} / \mathrm{m} 2)$. Le tabagisme était plus fréquent dans le G1 $(25,4 \%$ vs $16,6 \%)$. La rhinite allergique était plus fréquente dans le G1 ( $55,9 \%$ vs $41,6 \%)$, alors que l'hypertension $(57,6$ vs $62,5 \%)$ et le diabète $(23,7$ vs $41,6 \%)$ étaient plus fréquentes dans le G2. L'IAH était plus élevé dans le groupe $\mathrm{G} 2(26,7 / \mathrm{h}$ vs $34,3 / \mathrm{h}, \mathrm{p}=0,001)$. Le SAOS sévère $(45,76 \%$ vs $58,33 \%, p<10-3)$, la prescription d'une pression positive continue $(40,68 \%$ vs $60,42 \%, \mathrm{p}<10-3)$ et l'utilisation de corticostéroïdes inhalés à forte dose (57,63\% contre $81,25 \%, \mathrm{p}<10-3)$ étaient plus fréquents dans le groupe G2.

Conclusion: Les patients suivi pour un SAOS avec un asthme non ou partiellement contrôlé ont un SAOS plus sévère reflété par un IAH plus élevé nécessitant ainsi fréquemment un traitement par CPAP avec un recours plus fréquent à une corticothérapie inhalée à forte dose.

Conflits d'intérêt: Rien à déclarer
SSP/SSTS - EFP

Joint Annual Conference 2021 


\section{P168 \\ Pneumothorax et Pneumonie Virale Covid 19: Aspects Épidemiocliniques, Paracliniques et Pronostic à Propos de 8 Cas}

G.S. Moussounda, S. Ait Batahar, L. Amro

Pneumologie (Hôpital Arrazi, Chu Mohammed VI)

Introduction: Le pneumothorax spontané est une complication peu commune de la pneumonie virale COVID-19. L'incidence exacte et les mécanismes étiopathologiques sont encore inconnus.

Objectif: Énumérer les caractéristiques épidémiologiques, cliniques et paracliniques des patients présentant une association pneumothorax et Covid 19.

Patients et méthode: Il s'agit d'une étude descriptive, rétrospective allant de juillet à novembre 2020, menée à l'USI COVID-19 incluant les patients hospitalisés pour un pneumothorax associé à une pneumonie virale COVID-19 suspectée à l'imagerie et confirmé par PCR Covid 19.

Résultats: Au total 121 patients étaient enregistrés, parmi lesquels 8 étaient inclus (incidence 6,61\%). La moyenne d'âge était de 56,37 $\pm 14,48$ ans [31-77ans] avec une prédominance masculine (sex ratio $3 / 1$ ). Le tabagisme était noté pour $37,5 \%$, le contage tuberculeux pour $12,5 \%$. L'HTA (62,5\%), le diabète (50\%), l'asthme $(12,5 \%)$, la cardiopathie $(12,5 \%)$, la néoplasie $(12,5 \%)$ et l'insuffisance rénale $(12,5 \%)$ étaient les principales comorbidités. Les manifestations cliniques prédominantes étaient: la toux (100\%), la douleur thoracique (87,5\%), la fièvre (75\%), la dyspnée (75\%). La saturation en oxygène à l'air ambiant était de $83,87 \% \pm$ $4,64 \%$ [77 - 91\%] en moyenne. Sur le plan biologique on note une hyperleucocytose (100\%), une lymphopénie (87,5\%), la C-réactive protéine, les $\mathrm{LDH}$ et la ferritine étaient augmentées dans tous les cas (100\%), les D- dimères étaient élevées (75\%). Le pneumothorax était localisé à droite pour $50 \%(n=4)$, à gauche pour $37,5 \%(n=3)$ et bilatéral pour $12,5 \%(\mathrm{n}=1)$ avec un décollement complet pour $75 \%(n=6)$. On note la présence de verre dépolis dans tous les cas avec une atteinte parenchymateuse inférieure à 25\% retrouvée pour $12,5 \%(n=1)$, l'atteinte comprise entre $25-50 \%$ retrouvée pour $25 \%(n=2)$, l'atteinte entre $50-75 \%$ retrouvée pour $50 \%(n=4)$ et l'atteinte supérieure à $75 \%$ retrouvée pour $12,5 \%(n=1)$. Pour $75 \%$ les patients $(n=6)$ avaient un masque à haute concentration d'oxygène versus $25 \%(n=2)$ étaient sous ventilation non invasive. On note un retour du poumon à la paroi pour $87,5 \%(n=7)$. La durée moyenne d'hospitalisation était de $23 \pm 7$ jours et aucun décès n'a été rapporté.

Conclusion: Le pneumothorax peut être une complication rare de la pneumonie virale COVID-19. L'HTA et la toux était la comorbidité et la manifestation clinique prédominante avec un syndrome biologique inflammatoire et un décollement complet avec une atteinte comprise entre 50-75\%.

Conflits d'intérêt: Rien à déclarer

\section{P169 \\ Contribution de la Biopsie Pleurale à L'aveugle dans le Diagnostic des Pleurésies Exsudatives Lymphocytaires}

\section{G.S. Moussounda, S. Ait Batahar, L. Amro}

Pneumologie (Hôpital Arrazi, Chu Mohammed VI)

Introduction: La ponction biopsie pleurale (PBP) est une technique d'investigation à l'aveugle couramment utilisée en Afrique en raison d'une forte incidence de la tuberculose.

Objectif: Evaluer l'apport de la biopsie pleurale dans l'enquête étiologique des pleurésies exsudatives lymphocytaires.

Patients et méthodes: Il s'agit d'une étude descriptive, rétrospective allant du 01 janvier 2018 au 30 décembre 2019, incluant les patients reçus à l'hôpital du jour pour une pleurésie exsudative lymphocytaire. Pour tout test statistique le seuil de significativité retenu était la valeur de $\mathrm{p}<0,05$.

Résultats: $\mathrm{Au}$ total 239 patients étaient retenus, la moyenne d'âges était de 38,73 \pm 17,80 ans [16-95 ans], majoritairement de sexe masculin (sex ratio 1,9). L'aspect macroscopie jaune citrin et séro-hématique était retrouvé pour $90,79 \%(\mathrm{n}=217)$ et $9,21 \%$ $(n=22)$. L'histopathologie positive corrélé à l'aspect macroscopique jaune citrin ou séro-hématique était associé à la tuberculose pleurale pour $61,69 \%(n=124)$ et $21,05 \%(n=4)$ respectivement ( $p$ valu=0,0006). L'histopathologie positive corrélé à l'aspect macroscopique jaune citrin ou séro-hématique était associé à la néoplasie pour $2,80 \%(n=6)$ et $40,91 \%(n=9)$ respectivement $(\mathrm{p}$-valu<0,0001). La PBP à la première tentative était révélatrice de la tuberculose pour $77,17 \% \quad(n=98, S e=78,4 \%, S p=64,63 \%$, $\mathrm{VPP}=77,17 \%$, VPN=66,25\%, Index de Youden $=0,43$, p-valu $<0,0001)$ et d'une néoplasie pour $6,98 \%(n=9, S e=69,23 \%$, $\mathrm{Sp}=39,70 \%, \mathrm{VPP}=6,98 \%, \mathrm{VPN}=95,18 \%$, Index de Youden=0,09, $\mathrm{p}$-valu=0,3719). Par contre à la deuxième tentative la PBP était révélatrice de la tuberculose pour 33,75\% $(\mathrm{n}=27, \mathrm{Se}=21,60 \%$, $\mathrm{Sp}=35,37 \%, \mathrm{VPP}=33,75 \%, \mathrm{VPN}=22,83 \%$, Index de Youden $=-0,43$, $\mathrm{p}$-valu $<0,0001)$ et d'une néoplasie pour $4,82 \%(\mathrm{n}=4, \mathrm{Se}=30,77 \%$, $\mathrm{Sp}=60,30 \%, \mathrm{VPP}=4,82 \%, \mathrm{VPN}=93,02 \%$, Index de Youden $=-0,09$, $\mathrm{p}$-valu=0,3719). L'histologie des fragments retrouve un granulome avec nécrose caséeuse pour 53,55\% $(\mathrm{n}=128)$, un remaniement inflammatoire non spécifique pour $16,74 \%(\mathrm{n}=40)$, un granulome sans nécrose caséeuse pour $10,04 \%(\mathrm{n}=24)$, les lésions bénignes pour $3,76 \%(n=9)$, une origine non identifiée pour 9,62\% $(n=23)$, une lésion maligne pour $6,28 \%(n=15)$. Les lésions néoplasiques identifiées étaient un adénocarcinome pour $60 \%(n=9)$, un carcinome épidermoïde pour $26,67 \%(n=4)$ et un lymphome non hodgkinien pour $13,33 \%(n=2)$.

Conclusion: La biopsie pleurale à l'aveugle est une technique simple, peu invasive qui peut être proposée en première intention devant une pleurésie exsudative lymphocytaire. Elle était rentable pour $59,83 \%$ et a permis d'identifier la tuberculose et les lésions néoplasiques. Cependant la part non négligeable des pleurésies non étiquetées doit faire recourir à d'autres techniques d'investigations.

Conflits d'intérêt: Rien à déclarer 


\section{P170 \\ Le Carcinome Bronchique et L'infection Sars Cov-2 Chez les Patients Hospitalisés au Service de Pneumologie de Marrakech}

H. Benaziz, S. Abdala, S. Aitbatahar, L. Amro

Service de pneumologie. Hôpital Arrazi, CHU Mohamed VI FMPM, UCA, Marrakech, Maroc

Introduction: Le cancer bronchique constitue un problème majeur de santé publique. Cette épidémie à la COVID-19 a eu plusieurs impacts sur le diagnostic et la prise en charge de ses patients.

Matériels et méthodes: c'est une étude prospective incluant tous les cas de carcinomes bronchiques hospitalisés au service de pneumologie CHU Mohamed VI dont une PCR COVID-19 était positive entre Juin 2020 et Janvier 2021.

Résultats: on a colligé 44 cas de carcinome bronchique dont 6 avaient une PCR COVID-19 positive; tous les patients était des hommes, l'âge moyen était de 58 ans. Tous les malades étaient tabagique avec une moyenne de consommation de 36 PA. Le carcinome bronchique était associé à: un diabète de type II et une HTA ( 1 cas), une dilatation de bronche (1 cas). Les signes cliniques: toux avec expectorations blanchâtres: 5 cas (83\%), fièvre: 3 cas $(50 \%)$, douleur thoracique: 6 cas $(100 \%)$, hémoptysie 1 cas. Syndrome cave: 1 cas. Aucun patient n'a rapporté une agueusie et/ou une anosmie. Sur le plan radiologique un syndrome d'épanchement pleural liquidien a été retrouvé chez 2 cas; une opacité intraparenchymateuse chez 3 cas, une opacité médiastinopulmonaire chez 2 cas. La TDM thoracique a été réalisé chez tous les patients; dont un seul cas montrait une atteinte COVID-19 estimée 25-50\%; tous les patients étaient au minimum un stade IVa. 5 (83\%) cas ont été transféré et traité au chapiteau COVID-19; avec une bonne évolution clinique et sortie du chapiteau et référence en oncologie pour complément de prise en charge.

Conclusion: le carcinome bronchique ne semble pas être un facteur de gravité au même titre que les autres comorbidités; mais un dépistage systématique par PCR COVID semble utile pour détecter les formes asymptomatiques.

Conflits d'intérêt: Rien à déclarer

\section{P171 \\ La Valeur Pronostique de L'hyperleucocytose et Lymphopénie dans le Diagnostic de la Sévérité de L'infection SARS COV2}

\section{H. Benaziz, N. Ouakil, S. Ait Batahar, L. Amro}

Service de pneumologie, hôpital ARRAZI, CHU Mohamed VI, UCAM, FMPM, Marrakech.

Introduction: L'infection par le SARS COV 2 entraîne plusieurs manifestations quels soient cliniques biologiques et radiologiques. La numération de formule sanguine est un examen de routine qui peut évaluer la sévérité de l'atteinte
Méthode: Il s'agit d'une étude rétrospective et analytique concernant les patients hospitalisés en unité de soins intensifs du CHU Mohamed VI MARRAKECH pour infection SARS COV 2 et présentant une hyperleucocytose associée à une lymphopénie

Résultats: Parmi les patients hospitalisés, 27 patients présentaient une hyperleucocytose associée à une lymphopénie. La moyenne d'âge était de 59 ans, la majorité des patients étaient de sexe masculin $75 \%$, avec des co-morbidité telle que le diabète chez $40 \%$, hypertension artérielle $37 \%$. $7 \%$ des cas étaient asthmatiques. Tous les patients étaient symptomatiques, $77 \%$ présentaient des signes de gravité tel qu'une polypnée chez $66 \%$ des cas, une saturation inférieure à $94 \%$ chez $77 \%$. La confirmation de l'atteinte SARS COV 2 était par PCR chez $40 \%$ et par scanner thoracique $60 \%$. Sur le plan radiologique $30 \%$ des malades présentaient une atteinte sévère et $18 \%$ présentaient une atteinte critique, l'atteinte bilatérale présentait $74 \%$. Tous les patients présentaient un syndrome inflammatoire biologique. On a noté une amélioration de la ligne sanguine chez $30 \%$. Pour la prise en charge $48 \%$ ont été mis sous lunettes d'oxygène, $40 \%$ sous masque à haute concentration pour l'évolution clinique on a noté un seul décès, $30 \%$ des malades ont été transfert en réanimation et $66 \%$ ont sorti du chapiteau dont $37 \%$ avec extracteur d'oxygène.

Conclusion: L'association de l'hyperleucocytose et lymphopénie sont des indicateurs fiables sur la sévérité de l'atteinte chez les patients atteints par la COVID 19.

Conflits d'intérêt: Rien à déclarer

\section{P172 \\ Impact de la Pandémie COVID-19 sur les Résidents de Pneumologie Tunisiens}

MA. Jebali, H. Blibech, H. Snene, D. Belkhir, I. Wadhani, W. Fenina, N. Mehiri, N. Ben Salah, B. Louzir

Service de Pneumologie-Allergologie, CHU Mongi Slim, La Marsa, Tunis, Tunisie

Introduction: La pandémie d'OVID-19 a touché la plupart des structures de santé en Tunisie, en particulier lors de la deuxième vague. L'impact sur les résidents en médecine n'a pas été évalué. Notre objectif était d'évaluer l'impact du COVID-19 sur la formation des résidents en pneumologie, et leur bien être émotionnel.

Méthode: Un questionnaire en ligne a été diffusé sur une période d'une semaine en février 2021, évaluant la réduction du volume de cas et d'actes avant et pendant la deuxième vague de COVID-19 et incluant le score d'anxiété GAD-7 et le questionnaire de Burn Out. Les résidents en pneumologie inscrits de la 1ère à la 4ème année ainsi que ceux qui viennent de terminer leur cursus ont sollicités pour répondre au questionnaire.

Résultats: Parmi 80 résidents éligibles, 59 ont répondu au questionnaire. Parmi ceux-ci, $32,2 \%$ ont signalé une diminution de 50 à $75 \%$ du volume de cas observés. Concernant les actes, $22 \%$ des résidents pratiquent seulement 1 à 3 bronchoscopies par semaine, $27,1 \%$ n'effectuent aucune ponction pleurale et $61 \%$ n'effectuent aucun drainage thoracique. La plupart des résidents sont en unité COVID exclusive $(25,4 \%)$ ou en unités mixtes COVID et non 
COVID (47,5\%). Plus de la moitié des résidents (66.6\%) déclarent être extrêmement inquiets de l'impact de la pandémie COVID-19 sur leur formation. Près de la moitié des stagiaires souffraient d'anxiété modérée et de Burn Out modéré à sévère.

Conclusion: La pandémie COVID-19 a conduit à une réduction du volume de cas et d'actes pour les résidents en pneumologie, retentissant de façon considérable sur leur formation, avec un impact sur leur bien être émotionnel et leur épanouissement professionnel.

Conflits d'intérêt: Rien à déclarer

\section{P173 \\ L'exacerbation Aigue de la Bronchopneumopathie Chronique Obstructive: Un Véritable Tournant Évolutif}

\section{H. Mrassi, I. Bachouch, I. Nouira, N. Belloumi, NH. Khezami, C. Habbouria, F. Chermiti, S. Fenniche}

Service de pneumologie, Pavillon 4 de l'hôpital Abderrahmane Mami- Ariana

Introduction: La bronchopneumopathie chronique obstructive (BPCO) constitue un problème de santé publique de par sa fréquence et les dépenses qu'elle inflige. Son évolution est semée d'exacerbations aigues (EA) de sévérité variable.

But: Chercher les facteurs prédictifs d'indication d'une oxygénothérapie au long cours à domicile (OLD) au décours d'une hospitalisation pour EA

Méthodes: Étude rétrospective sur les dossiers de patients (pts) suivis pour BPCO au Pavillon 4 de l'hôpital A. Mami et hospitalisés au moins une fois entre 2016-2019. Les pts déjà sous OLD étaient exclus.

Résultats: Cent pts de sexe masculin colligés (âge moyen= $68 \mathrm{ans})$. La moitié des pts avaient au moins une comorbidité cardiovasculaire. Les pts étaient classés GOLD C ou D dans $54 \%$ des cas. Le VEMS moyen en post bronchodilatation était de $1160 \mathrm{~mL}$ [5002200]. La pression artérielle moyenne en $\mathrm{O} 2$, à l'admission, était de $52 \mathrm{~mm} \mathrm{Hg}$. Un examen cytobactériologique des crachats était réalisé chez $75 \%$ des pts. Un germe était isolé chez 40 pts avec une prédominance du pneumocoque $(n=25)$. La durée moyenne d'hospitalisation était de $12 \pm 4$ jours. Une ventilation non invasive (VNI) était indiquée dans $20 \%$ des cas durant le séjour et dans $14 \%$ des cas à la sortie. Une OLD était prescrite chez 19 pts (19\%). Cette prescription était plus fréquente en cas de cardiopathie sousjacente $(\mathrm{p}=\mathrm{NS})$, d'un profil exacerbateur fréquent $(\mathrm{p}=0,02)$ et d'un recours à la VNI durant l'hospitalisation $(\mathrm{p}=0,01)$. Un séjour hospitalier prolongé s'associait à une sortie sous $\operatorname{OLD}(\mathrm{p}<0,001)$. L'isolement d'un germe était plus observé chez les pts sortis sous OLD ( $\mathrm{p}=\mathrm{NS})$.
Conclusion: Selon nos résultats, le profil exacerbateur fréquent; le recours à la VNI et le séjour hospitalier prolongé sont prédicteurs de l'évolution vers l'insuffisance respiratoire chronique au décours d'une EA de BPCO.

Conflits d'intérêt: Rien à déclarer

\section{P174 \\ Impact de L'insuffisance Pondérale sur la Sévérité de la Bronchopneumopathie Chronique Obstructive}

\author{
H. Mrassi, I. Bachouch, I. Nouira, N. Belloumi, NH. Khezami, \\ C. Habbouria, F. Chermiti, S. Fenniche
}

Service de pneumologie, Pavillon 4 de l'hôpital Abderrahmane Mami- Ariana

Introduction: La bronchopneumopathie chronique obstructive (BPCO) est une maladie systémique pourvoyeuse de complications diverses, dont la dénutrition.

But: Evaluer l'impact de l'insuffisance pondérale sur la sévérité de la maladie.

Méthode: Étude rétrospective sur les dossiers de patients (pts) suivis pour BPCO au Pavillon 4 de l'hôpital A. Mami [2016-2019]. Deux groupes étaient comparés: $\mathrm{Gl}=$ Indice de masse corporelle $(\mathrm{IMC})<18,5 \mathrm{~kg} / \mathrm{m} 2 ; \mathrm{G} 2=\mathrm{IMC} \geq 18,5 \mathrm{~kg} / \mathrm{m} 2$.

Résultats: Cent pts de sexe masculin colligés (âge moyen = $68 \mathrm{ans})$. Ils étaient tous tabagiques. Les comorbidités les plus retrouvées étaient: HTA (21\%) et le diabète (14\%). L'IMC moyen était de $24 \mathrm{~kg} / \mathrm{m} 2$. Trente-sept pts (37\%) étaient maigres. Les pts étaient classés: GOLD B (46\%), GOLD C (20\%) et GOLD D (34\%). Dans le groupe GOLD D, les pts du G1 étaient plus nombreux ( $58 \%$ vs $41 \%, p=0,02)$. Le VEMS moyen en post bronchodilatation était de: [G1: $0,9 \mathrm{~L} ; 35 \%]$ vs $[1,4 \mathrm{~L} ; 51 \%] ; \mathrm{p}<0,001$. L'indice de BODE était plus élevé dans le G1 ( $\mathrm{p}=0,02)$. La pression artérielle moyenne en $\mathrm{O} 2$ à l'état stable, était de $60 \mathrm{~mm} \mathrm{Hg}$ dans le G1 vs 71 dans le G2, p=NS. Soixante-dix pourcent des pts du G1 étaient exacerbateurs fréquents vs $38 \%$ pour le $\mathrm{G} 2 ; \mathrm{p}=0,01$. Le nombre annuel moyen d'hospitalisations était de: [G1: 2; G2: 1; $p=0,04]$. L'observance du traitement inhalé était comparable dans les deux groupes; $\mathrm{p}=\mathrm{NS}$. La ventilation non invasive à domicile était plus retrouvée dans le G1 $(n=9,24.3 \%)$ vs $(n=5,8 \%)$ pour le $G 2 ; p=N S$.

Conclusion: Selon nos résultats, l'insuffisance pondérale s'associe à une maladie plus sévère. Une prise en charge nutritionnelle adaptée est nécessaire afin d'améliorer le pronostic de nos malades

Conflits d'intérêt: Rien à déclarer 


\section{P175 \\ Predictors of COVID-19-Related Mortality: Experience of the Covid-19 Unit at Mahdia University Hospital}

Baili Hassène, Touil Imen, Brahem Yosra, Ayeb Jihène, Ksissa Souhir, Boudawara Keskes Nadia, Boussoffara Leila, Knani Jalel

Pulmonology department, University hospital center TAHER SFAR, Mahdia, TUNISIA

Introduction: the novel coronovirus infection has become a major healthcare concern wich requires prompt cncerned and subtained effort. Its seriousness lies in the risk of developing in $20 \%$ of cases a cascade of reactions that can lead to death.

Aim: to assess risk factors for covid-19 mortality

Methods: retrospective study including 161 patients admitted to our covid unit between july 2020 and Mars 2021. Diagnostic confirmation was obtained either by RT-PCR or rapid test.

Results: the mean age was 63 years +-13 , with male predominance. Hypertension was present in $55 \%$ of cases, $40 \%$ were known diabetics. Nine patients have chronic renal failure including 2 in the dialysis stage. Respiratory disease was found in $13.2 \%$ of our patients. The average of consultation period was 4.2 days. Mortality reaches $19 \%$ among our patients. The major correlation factors with mortality were: intensive care stay $(\mathrm{p}=0.00001)$, diabetes $(p=0.028)$, patients long-term oxygen $(p=0.031)$, the presence of flu syndrome $(\mathrm{p}=0.0001)$ and respiratory distress signs $(\mathrm{p}=0,0001)$ as well as a high oxygen need initially $(\mathrm{p}=0.0001)$. No significant correlation was observed for biology or treatment. In multivariate analysis only the need for a high flow of oxygen initially and the need for resuscitation were the independent factors of mortality.

Conclusion: the need for a high flow of oxygen on admission as well as the need for resuscitation seem to be the main factors of mortality in our patients regardless of any comorbidity

\section{P176 \\ Caractéristiques des Exacerbations Aiguës de Broncho Pneumopathie Chronique Obstructive Avec Hyperéosinophilie}

\section{H. Cherif, S. Debbiche, F. Yangui, B. Karaborni, M. Triki, MR. Charfi}

Service de pneumologie, Hôpital FSI

Introduction: L'hyperéosinophilie des voies respiratoires connue caractéristique de l'asthme, est désormais un marqueur de l'inflammation dans la BPCO et peut être attesté également par la présence d'une hyper éosinophilie sanguine. Les exacerbations aigües de BPCO (EABPCO) sont assez hétérogènes.

L'objectif de notre travail était d'identifier les EABPCO avec hyperéosinopilie et de déterminer leurs caractéristiques cliniques et évolutives.
Méthode: Etude rétrospective menée entre mars 2014 et décembre 2019 ayant inclus 78 dossiers de patients hospitalisés pour EABPCO. Deux groupes (G1 et G2) ont été individualisés selon la présence ou non d'une hyperéosinophilie sanguine définie par un taux de polynucléaires éosinophiles $\geq 200 / \mathrm{mm} 3$. G1: patients avec une hyperéosinophilie, $(30,8 \%)$ et G2: patients sans hyperéosinophilie $(69,2 \%)$.

Résultats: L'âge moyen était de $68 \pm 9$ ans. Tous nos patients étaient tabagiques avec une consommation moyenne de 65 PA. L'analyse des paramètres de sévérité des EABPCO montrel'absence de différence significative entre les 2 groupes concernant les paramètres cliniques: la polypnée $(\mathrm{p}=0,49)$, la tachycardie $(0,17)$, les signes de lutte $(0,6)$, et les signes d'encéphalopathie respiratoire $(\mathrm{p}=1)$. Les paramètres gazométriques à l'admission: la $\mathrm{PaO} 2$ $(62,5 \mathrm{mmHg}$ vs $59,8 \mathrm{mmHg} ; \mathrm{p}=0.33)$ et la capnie $(42,9 \mathrm{mmHg}$ vs $46,7 \mathrm{mmHg} ; \mathrm{p}=0.12$ ) étaient également comparables entre les deux groupes. La biologie a montré une leucocytose comparable $(\mathrm{p}=0,29)$, une lymphocytose plus importante $(2020,9 / \mathrm{mm} 3$ vs $1512 / \mathrm{mm} 3, \mathrm{p}=0,001)$ et un taux de CRP plus bas dans le G1 $(64,7$ $\mathrm{mg} / \mathrm{l}$ versus $118 \mathrm{mg} / \mathrm{l}, \mathrm{p}=0,025)$. Le recours à la corticothérapie systémique $(87,5 \%$ vs $92,2 \% ; \mathrm{p}=0,67)$ et la durée moyenne d'hospitalisation ( 12,71 jours vs 11,75 jours; $\mathrm{p}=0,65)$ étaient comparables entre les 2 groupes. A la sortie, la gazométrie a montré une capnie $(42,6 \mathrm{mmHg}$ vs $48,37 \mathrm{mmHg}$; $\mathrm{p}=0,023)$ et un taux de bicarbonates $(27,15 \mathrm{mmHg}$ vs $30,18 \mathrm{mmHg}$; $=0,003)$ plus bas dans le G1. $\mathrm{La} \mathrm{PaO} 2$ à la sortie était comparable entre les deux groupes $(\mathrm{p}=0,26)$.

Conclusion: L'augmentation du taux d'Eosinophiles $\geq 200 /$ $\mathrm{mm} 3$ chez les patients BPCO ne semble pas avoir une influence au cours des EABPCO

Conflits d'intérêt: Rien à déclarer

\section{P177 \\ Apport du Quantiferon-TB Gold Plus Dans le Diagnostic de la Tuberculose Maladie}

\section{Hicham Naji-Amrani, Youssef Bougrini, Reda Belghol, Aziz Ouarssani}

Service de Pneumo-phtisiologie, Hôpital Militaire Moulay Ismail, Meknès, MAROC

Introduction: Le Quantiféron-TB Gold plus (QTF plus), développé en 2016, est un test de libération de l'interféron-gamma qui comporte deux valeurs TB1 et TB2 qui estiment la réponse des lymphocytes T CD8 corrélée à la Tuberculose (TB) active permettant de la différencier de la TB infection. L'objectif de ce travail est d'évaluer la valeur diagnostic du dosage de QTF-Plus dans une population de patients Marocains suspect de tuberculose active et de déterminer des seuils de positivité permettent d'éliminer ou de consolider le diagnostic de TB active.

Méthode: Des patients suspects de TB maladie ont été recrutés avec établissement du diagnostic sur faisceaux d'arguments, les valeurs du Qtf plus ont permis de réaliser les courbes de ROC et définir la sensibilité (Se) spécificité (Sp) valeur prédictive positive 
(VVP) et valeur prédictive négative (VPN) des différents paramètres en plus des seuils de positivité.

Résultats: Quarante-sept patients (29 hommes/18 femmes) ont été recrutés, l'âge moyen est de 43,2 ans. 32 patients $(68,1 \%)$ avaient une TB maladie, alors que 15 malades $(31,9 \%)$ ne présentent pas de TB. La valeur de TB2 était plus précise pour le diagnostic de TB maladie avec un seuil de positivité de $0.54 \mathrm{UI} / \mathrm{ml}$, une aire sous la courbe à $90.9 \%$, Se à $96.9 \%$, Sp à $80 \%$, VPP à $88.57 \%$ et VPN à 91.67\%. Dans notre série, si la valeur la plus élevée de TB1 et TB2 est inférieure à $0.11 \mathrm{UI} / \mathrm{mL}$ le diagnostic de TB pourrait être éliminé (La Se et la VPN s'élèvent à 100\%). Alors qu'une réponse TCD8 estimée par la valeur de TB2-TB1 supérieur à $0.5 \mathrm{UI} / \mathrm{mL}$ permettrait de consolider le diagnostic de TB évolutive (Sp et VPP à $100 \%)$.

Conclusion: Le Qtf plus garde une place importante dans le diagnostic de la TB maladie. Sa Sp et Se peuvent être améliorées par une variation du seuil de positivité.

Conflits d'intérêt: Rien à déclarer

\section{P178 \\ Observance Thérapeutique Chez Les Patients Asthmatiques au Cours de la Pandémie COVID19}

\author{
H. Snène, K. Zayen, H. Blibech, W. Fenina, I. El Wadhane, A. Jebali, \\ J. Daghfous, N. Mehiri, N. Ben Salah, B. Louzir
}

Université de Tunis El Manar, Faculté de Médecine de Tunis, CHU Mongi Slim, Service de Pneumologie Allergologie, 2070 Sidi Daoud, La Marsa, Tunisie

Introduction: La pandémie de la COVID-19 a rendu l'accès aux soins difficiles durant l'année 2020 pour les patients ayant une pathologie respiratoire chronique. Le but de notre travail était d'évaluer l'observance thérapeutique chez les patients asthmatiques durant cette pandémie.

Méthode: Etude transversale menée au service de pneumoallergologie du CHU Mongi Slim La Marsa, à Tunis en Tunisie, durant le mois de Janvier 2021, portant sur les patients asthmatiques adultes et dont le suivi de la maladie asthmatique a débuté durant l'année 2018 avec des consultations régulières (>2) au cours des années 2018 et 2019. Le diagnostic d'asthme a été retenu selon les recommandations du GINA 2018. Les patients ayant d'autres pathologies respiratoires chroniques associées ont été écartés. Le contrôle de l'asthme a été déterminé selon les recommandations du GINA 2020 et l'observance thérapeutique évaluée par le questionnaire de Girerd composé de 6 questions (bonne observance si toutes les réponses sont non et mauvaise observance si $\geq 3$ oui). Tous les patients ont été interrogés par téléphone.

Résultats: Un total de 249 dossiers de patients asthmatiques a été consulté, 88 ont été contacté par téléphone et 54 ont répondu au questionnaire. L'âge moyen était de $53 \pm 14$ ans avec un sexratio $(F / H)=1,34$. Le traitement de fond était basé sur les SABA seuls dans $6 \%$ des cas, les CI dans $20 \%$ et l'association CI et LABA dans $74 \%$. Un arrêt temporaire du suivi durant l'année 2020 a été constaté chez $62 \%$ des patients et un arrêt définitif dans $6 \%$. Les principales causes d'arrêt étaient: la peur de la contamination par la COVID-19 dans $42 \%$ des cas et l'absence de symptômes dans $12 \%$. Selon le questionnaire de Girerd, 19\% des patients étaient bon observants, $37 \%$ modérément observants et $44 \%$ non observants. L'arrêt du traitement durant l'année 2020 a été retrouvé dans $50 \%$ des cas avec une durée moyenne d'arrêt de 56,86 jours. Les principales causes étaient: l'absence de symptômes (46\%), l'impossibilité de renouveler l'ordonnance (29\%) et les problèmes financiers (11\%).

Conclusions: Durant l'année 2020, 44\% des patients étaient mal observants de leur traitement avec un arrêt de suivi constaté dans $62 \%$ des cas principalement par peur de la contamination virale.

Conflits d'intérêt: Rien à déclarer

\section{P179 \\ Impact de L'hyperéosinophilie Sanguine sur le Pronostic de la BPCO}

Haifa Zaibi, Ines Laouini, Emna Ben Jemia, Jihen Ben Amar, Hichem Aouina

Service de pneumologie - hôpital charles nicolle de Tunis

Introduction: La BPCO est considérée actuellement comme une véritable maladie systémique responsable d'une inflammation systémique. Le profil inflammatoire, bien que classiquement de type neutrophilique, définit différents phénotypes de BPCO dont le phénotype éosinophilique. L'éosinophilie sanguine serait associée à un risque plus important d'exacerbation. Aurait-elle un impact sur le pronostic de la BPCO?

Objectif: Etudier l'association entre l'hyperéosinophilie et le pronostic de la BPCO

Méthodes: Nous avons mené une étude prospective et analytique, au service de pneumologie de l'hôpital Charles Nicolle de Tunis. Les patients inclus étaient évalués à l'état stable et suivis sur une période de 12 mois. Ils ont tous bénéficié d'une Numération de la Formule Sanguine afin de dépister l'hyperéosinophilie, définie dans notre série par un taux d'éosinophiles de 200 éléments/ $\mathrm{mm} 3$ ou plus.

Résultats: Nous avons inclus 120 patients d'âge moyen de $65,3 \pm 11$ ans, avec prédominance masculine (sex ratio=8,2). L'éosinophilie sanguine moyenne était de 182 éléments/mm3. Une hyperéosinophilie a été retrouvée chez 49 patients (40,8\%). L'hyperéosinophilie était plus associée aux stades avancés de la BPCO (Groupe D) $(\mathrm{p}=0,046)$. En étudiant les paramètres de l'indice de BODE, aucune corrélation n'a été notée entre l'éosinophilie et l'IMC $(\mathrm{p}=0,4)$, ni avec la sévérité de l'obstruction bronchique évaluée par le VEMS $(\mathrm{p}=0,5)$. De même, la sévérité de la dyspnée évaluée selon l'échelle mMRC était comparable chez les patients présentant ou non une hyperéosinophilie $(\mathrm{p}=0,28)$. Aussi, le taux d'éosinophiles n'était pas significativement corrélé à la distance parcourue $(p=0,4)$. Ainsi, la classification selon le score de BODE n'était pas impactée par l'éosinophilie sanguine $(\mathrm{p}=0,15)$. Aussi, le taux d'éosinophiles sanguin n'était pas corrélé à l'indice de BODE calculé $(\mathrm{p}=0,5)$ (tableau). 
Conclusion: Conformément à la littérature tunisienne, l'hyperéosinophilie sanguine ne semblait pas impacter le pronostic de la BPCO, en particulier, en termes de dyspnée, de sévérité de l'obstruction bronchique et de tolérance à l'effort.

Conflits d'intérêt: Rien à déclarer

\section{P180 \\ Forme Pseudo Tumorale de la Tuberculose Broncho-Pulmonaire à Propos de 23 Cas}

Iqraoun Siham

Service de Pneumologie CHU Hassan II Fès. Maroc

Introduction: La tuberculose pulmonaire pseudo tumorale est une entité rare, elle peut simuler un cancer surtout chez le tabagique.

Méthode: Il s'agit d'une étude rétrospective réalisée au sien du service de pneumologie du CHU Hassan II de Fès, sur une période de 5 ans du juillet 2015 au janvier 2021.

Résultats: Nous avons colligé 23 cas: 18 hommes et 5 femmes, L'âge moyen était de 56 ans (extrêmes 31 et 81 ans). Le tabagisme était retrouvé chez $65 \%$ des patients. L'antécédent de tuberculose était retrouvé chez 4 patients, le contage tuberculeux chez 3 patients, le diabète chez 3 malades, une patiente était suivie pour cancer du col sous radio chimiothérapie et une patiente était en insuffisance rénale chronique sous dialyse. La symptomatologie fonctionnelle respiratoire était dominée par les signes généraux dans tous les cas, la toux chronique et la dyspnée dans $65 \%$ des cas. L'examen clinique était normal chez $57 \%$ des patients, avait objectivé un syndrome d'épanchement liquidien associé dans $9 \%$ des cas, La radiographie thoracique montrait une opacité d'allure tumorale dans 17 cas. Le scanner thoracique avait montré une masse tissulaire dans 10 cas, associée à des adénopathies médiatinales dans 8 cas, un nodule isolé suspect dans 5 cas, une lésion de condensation dans 7 cas et une atélectasie dans un seul cas, la localisation la plus fréquente est LSG. la recherche de BK sur les expectorations (ED et genexpert) était négative dans tous les cas. La fibroscopie bronchique a été réalisé chez tous les patients et avait montré un bourgeon endobronchique dans 2 cas, une sténose infiltrante dans 4 cas, un épaississement endobronchique chez 4 patients, une inflammation bronchique dans 2 cas, une compression extrinsèque dans un seul cas, des tâches d'anthracose dans 3 cas et un aspect normal 10 cas. Le diagnostic de la tuberculose pulmonaire était confirmé par la biopsie bronchique dans 4 cas, la biopsie scannoguidée chez 8 cas, la biopsies chirurgicales 7 cas (résection atypique dans 2 cas, une lobectomie dans 1 seul cas et chirurgie d'exérèse nodulaire dans 4 cas), la bacilloscopie et le genexpert dans le liquide d'aspiration bronchique chez 4 cas. L'évolution sous traitement antibacillaire était favorable chez environ $90 \%$ des cas.

Conclusion: Le diagnostic de la tuberculose doit être évoqué devant toute atteinte pulmonaire même d'allure tumorale surtout dans un contexte épidémiologique et clinique évocateur. Le principal risque étant de méconnaître une pathologie curable par un traitement médical spécifique.

Conflits d'intérêt: Rien à déclarer

\section{P181 \\ Eosinopénie: facteur de Mauvais Pronostic des Infections à covid19?}

\section{N. Boubaker, I. Mejri, S. Mhamdi, S. Dabboussi, C. Aichaouia, Z. Moatemri, M. Khadhraoui}

Service de pneumologie- Hôpital Militaire principal d'instruction de Tunis

Introduction: L'éosinopénie a été observée chez les patients atteints de COVID19. Son apport dans le dépistageet le diagnostic de la maladie a été bien démontré. Cependant son impact sur la sévérité et le pronostic de la maladie reste controversé. L'objectif de notre étude était d'étudier la relation entre l'éosinopénie à l'admission et la sévérité et le pronostic de la COVID19.

Méthode: Il s'agit d'une étude rétrospective incluant des patients hospitalisés au service de pneumologie de l'hôpital militaire principal d'instruction de Tunis. Nous avons défini deux groupes en fonction du taux des éosinophiles: Groupe $1(\mathrm{~N}=88)$ : $\mathrm{PNE}<40 \mathrm{el} / \mathrm{mm} 3$; Groupe 2 ( $\mathrm{N}=52): \mathrm{PNE} \geq 40 \mathrm{el} / \mathrm{mm} 3$.

Résultats: Nous avons inclus 140 patients. L'âge moyen était de $61 \pm 13$ ans. L'hypertension artérielle était la comorbidité la plus fréquente $(39,1 \%)$ suivie par le diabète (38\%)et l'asthme (10\%). Les signes cliniques à l'admission étaient dominés par la fièvre (65\%), l'asthénie (61\%) et la dyspnée (34\%). Les signes de lutte étaient présents essentiellement chez les patients du premier groupe $(\mathrm{p}=0.03)$. Le taux bas d'éosinophiles était associé à des besoins plus élevés en oxygène à l'admission $(\mathrm{p}=0.02)$ ainsi qu'une atteinte parenchymateuse plus sévère à la tomodensitométrie $(\mathrm{p}=0.004)$. Par ailleurs, notre étude n'a pas montré d'association entre un taux bas d'éosinophiles et le transfert au service de réanimation $(\mathrm{p}=0.6)$ ainsi que la mortalité $(\mathrm{p}=0.9)$.

Conclusion: Selon notre étude, l'éosinopénie semble être associée à la sévérité de la COVID19. D’autres études à plus grande échelle seraient nécessaires afin de bien étudier cette relation.

Conflits d'intérêt: Rien à déclarer

\section{P182 \\ Comorbidités et Saos: Prévalence Selon le Sexe}

H. Mrassi, I. Mejri, S. Mhamdi, S. Dabboussi, C. Aichaouia,

Z. Moatemri, M. Khadhraoui

Service de pneumologie- Hôpital Militaire principal d'instruction de Tunis

Introduction: Le syndrome apnées-hypopnées obstructives du sommeil (SAOS) constitue le trouble respiratoire nocturne le plus fréquent. Il représente un véritable problème de santé publique notamment en raison des comorbidités qui lui sont associées.

On se propose de rechercher une différence de la prévalence de ces comorbidités selon le sexe.

Méthode: Etude rétrospective menée sur les dossiers médicaux des patients ayant bénéficié d'une polygraphie ventilatoire dans notre service, entre 2017 et 2019, et chez qui le diagnostic de SAOS 
était retenu: Selon l'index apnées-hypopnées (IAH), les pts étaient classés en SAOS léger (5-15), modéré (16-30) et sévère $(>30)$. Deux groupes étaient comparés: G0 (Hommes) et G1 (Femmes).

Résultats: Cent-cinquante patients étaient inclus: [G0: 66 hommes]; [G1:84 femmes]. L'âge moyen en années était de: $\mathrm{G} 0=43,5 \pm 10,8$ vs $\mathrm{G} 1=57,2 \pm 11 ;(\mathrm{p}<10-4)$. Le tabagisme était noté chez 58 hommes $(87 \%)$ vs huit femmes $(0,9 \%)$; $(\mathrm{p}<10-4)$. Le SAOS était classé modéré à sévère chez $80 \%$ des hommes vs $67,8 \%$ des femmes; $(\mathrm{p}<10-4)$. Dans le G0, 25 patients $(37,8 \%)$ avaient au moins deux comorbidités cardiovasculaires vs 61 pts $(72,6 \%)$ dans le G1; $(p<10-4)$. L'étude de la prévalence des comorbidités dans les deux groupes montre que les indicateurs suivants étaient significativement plus fréquents chez les femmes: obésité ( $87 \%$ versus $68 \%, \mathrm{p}=0,04)$, HTA ( $66 \%$ versus $28 \%, \mathrm{p}<0,01)$, dyslipidémie $(32 \%$ versus $13 \%, p=0,008)$ et dysthyroidie $(23 \%$ versus $1 \%, p<0,01)$. Le diabète et l'insuffisance coronaire étaient notés dans les deux groupes sans différence significative. Un traitement combiné à base d'une cure d'amaigrissement associée à un appareillage par CPAP était indiqué chez 45 hommes (68\%) et 36 femmes $(42,8 \%)$. Au cours du suivi, le taux d'observance thérapeutique était meilleur dans le G0: $88 \%$ vs 50\%, $\mathrm{p}<0,05)$.

Conclusion: Selon nos résultats; le SAOS de la femme prédomine à un âge post ménopausique et se classe modéré à sévère dans les deux tiers des cas. Il s'associe à un risque cardiovasculaire accru; en plus des pathologies respiratoires obstructives chroniques et des dysthyroïdies. De ce fait, il est impératif de prendre en charge ces comorbidités afin de réduire la morbi-mortalité associée.

Conflits d'intérêt: Rien à déclarer

\section{P183 \\ Apport de L'échographie Thoracique dans le Choix du Site de la Ponctionpleurale}

\section{Mejri, T. Znegui, S. Mhamdi, S. Dabboussi, C. Aichaouia, Z. Moatemri, M. Khadhraoui}

Service de pneumologie- Hôpital Militaire principal d'instruction de Tunis

Introduction: Depuis 2010, la British Thoracic Society recommande le guidage échographique des procédures pleurales afin d'améliorer leur sécurité et leur rentabilité diagnostique. Le but de notre étude était d'évaluer l'apport de l'échographie thoracique dans le choix du site de la ponction pleurale.

Méthode: Etude monocentrique transversale évaluative faisant inclure 43 patients hospitalisés au service de pneumologie de l'hôpital militaire, entre janvier et Novembre 2020, pour exploration d'une opacité d'allure pleurale sur la radiographie du thorax. On a évalué la concordance entre les repères cliniques proposés par deux groupes de médecins (Seniors et Juniors). Puis un médecin expérimenté en échographie thoracique a vérifié l'exactitude des repères cliniques des deux groupes et les repères échographiques proposés par les juniors.

Résultats: La moyenne d'âge était de 60,5 \pm 17,5 ans. L'échographie a confirmé la présence d'un épanchement pleural dans 38 cas (84\%). Elle a éliminé dans $16 \%$ des cas un diagnostic différentiel: une pachypleurite $(n=4)$ et une atélectasie $(n=3)$. A l'échographie thoracique, l'épanchement était majoritairement libre anéchogène $(n=33)$, versus cloisonné $(n=4)$. Il était de moyenne abondance dans presque la moitié des cas $(n=20)$. Comparées à l'échographie thoracique, la sensibilité du repérage clinique et celle de la radiographie thoracique dans le diagnostic positif de pleurésie étaient estimées respectivement à $74 \%$ et $83 \%$. Le taux d'erreur de repérage clinique était significativement plus élevé chez les juniors, estimé à $77 \%$ versus $49 \%$ chez les seniors $(\mathrm{p}<0,05)$. Trente et un sites $(57 \%)$ correspondaient à des sites dangereux: poumon $(n=11)$, foie $(n=10)$, diaphragme $(n=7)$ et rate $(n=3)$. Les facteurs de risque d'erreurs révélés étaient: le côté droit et l'aspect d'un épanchement de faible abondance à la radiographie thoracique avec un risque relatif (RR) estimé respectivement à 1,44 $[0,56-3,72]$ et $1,82[0,52-6,40]$. L'expérience des seniors avait un impact positif sur le choix des repères cliniques, avec une concordance modérée (Kappa: 0,4-0,6). La courbe d'apprentissage des juniors avait montré que le taux d'erreur diminuait avec le nombre d'échographies réalisées notamment chez ceux qui avaient bénéficié d'une formation théorique au préalable $(n=32)$.

Conclusion: L'échographie thoracique améliore significativement la rentabilité diagnostique des ponctions pleurales et diminue le risque decomplicationsindépendamment duniveau d'expérience de l'opérateur.

Conflits d'intérêt: Rien à déclarer

\section{P184 \\ Particularités de la Pneumonie COVID-19 Chez les Sujets Âgés: Etude Comparative}

\author{
Medi wafa, Cherif Intissar, Kharchoufi Kamel, Bendayekh afef, \\ El Ghoul Jamel
}

Service de Pneumologie/ service ORL/ service des urgences

Introduction: La pandémie de COVID-19 constitue actuellement le plus grand défi auquel le système sanitaire est confronté, et dans ce cadreles sujets âgés en particulier sont objets d'inquiétude et de préoccupation.

But: Etudier les particularités cliniques, radiologiques et évolutives de la pneumonie COVID-19 chez les sujets âgés.

Patients et méthode: étude rétrospective portant sur 213 patients hospitalisés et pris en charge pour pneumonie COVID-19, au service de pneumologie du CHU Habib Bourguiba Médenine entre Septembre 2020 et Mars 2021. Les patients ont été répartis en deux groupes: G1 (âge $>65$ ans, $n=123$ ), G2 (âge $\leq 65$ ans, $n=90$ ).

Résultats: L'âge moyen était de $78 \pm$ ans dans le $\mathrm{G} 1$ et $54 \pm$ ans dans le G2. Dans les deux groupes, il existait une prédominance masculine avec un sexe ratio de 1.19 et 1.30 dans G1 et G2 respectivement. L'intoxication tabagique était retrouvée chez $15 \%$ des patients dans G1. Le diabète domine les comorbidités étudiées, il était noté chez $48 \%$ des cas (G1) versus $32 \%$ des cas (G2) ( $p=0.6)$. Le profil radiologique était similaire dans les deux groupes, dominé par l'atteinte étendue au scanner thoracique. Dans le G1, 32,5\% des patients avaient une atteinte sévère à critique versus $36,8 \%$ dans le G2. La durée d'hospitalisation était plus longue dans le G1 
(9,6 jours vs 8,3 jours). Les complications thromboemboliques étaient plus fréquentes dans le G2 (10\% versus $4,8 \%, \mathrm{p}=0,12)$, alors que $6,5 \%$ des patients du G1 avaient présenté des troubles neurologiques. Le transfert au service de réanimation était indiqué dans 13,8\% (G1) et 20\% (G2). La mortalité était plus élevée dans le G1, soit $20,2 \%$ contre $4,4 \%$ dans le G2 (p<0.001). Des séquelles à type de fibrose pulmonaire étaient observées dans les deux groupes $(7,3 \%$ vs $7,7 \%, p=1,12)$.

Conclusion: Il ressort de cette étude que d'une part l'âge avancé est associé à une mortalité élevée, d'autre part, même chez les sujets plus jeunes, la pneumonie COVID-19 reste une affection grave qui peut nécessiter un séjour au service de réanimation, se compliquer d'une embolie pulmonaire et garder des séquelles à type de fibrose pulmonaire.

Conflits d'intérêt: Rien à déclarer

\section{P185 \\ Expérience D’un Service de Pneumologie Tunisien dans la Prise en Charge des Patients COVID-19}

\author{
Medi wafa, Cherif Intissar, Kharchoufi Kamel, Bendayekh afef, \\ El Ghoul Jamel \\ Service de Pneumologie/ service ORL/ service des urgences
}

Introduction: La propagation du nouveau coronavirus SARSCoV-2, découvert en Chine en janvier 2020, a touché le monde entier et a atteint la Tunisie et plus particulièrement notre région dès Juillet 2020.

Objectif: Déterminer les caractéristiques cliniques, radiologiques et évolutives des patients hospitalisés pour COVID-19.

Patients et Méthode: Etude rétrospective portant sur 213 dossiers de patients COVID-19 hospitalisés au service de pneumologie à l'hôpital Habib Bourguiba Médenine entre Septembre 2020 et Mars 2021.

Résultats: Il s'agissait de 118 hommes et 95 femmes, d'âge moyen de $68 \pm 14$ ans avec des extrêmes allant de 25 à 110 ans. Une intoxication tabagique était retrouvée chez $13.9 \%$ des patients, alors que 3\% seulement étaient des fumeurs actifs. Les comorbidités étaient dominées par l'HTA (44.1\%) et le diabète (37,6\%). $21.6 \%$ des patients étaient obèses. Les symptômes étaient dominés par la dyspnée de repos dans 70,4\% suivie par l'asthénie (51,6\%). Le diagnostic positif était basé sur les tests virologiques et antigéniques dans $83,5 \%$ des cas. Le scanner thoracique, pratiqué chez 147 patients, a révélé une atteinte étendue à sévère dans $75,5 \%$ des cas. La prise en charge était basée sur l'oxygénothérapie et la corticothérapie systémique chez tous les malades. La ventilation non invasive était utilisée chez $9,9 \%$ des patients. Les complications précoces étaient dominées par l'embolie pulmonaire dans $7 \%$ des cas. Un transfert au service de réanimation était indiqué dans $16,4 \%$ des cas. La durée moyenne d'hospitalisation était de $11 \pm 7$ jours avec une mortalité moyenne estimée à 16,4\%.
Conclusion: La pneumonie causée par le SARS-CoV-2 est une affection grave à l'origine d'une mortalité élevée. La connaissance de ses caractéristiques cliniques et évolutives pourrait optimiser sa prise en charge dans notre région.

Conflits d'intérêt: Rien à déclarer

\section{P186 \\ Le Syndrome D’obésité Hypoventilation: À Propos de 28 Cas}

\section{EL Ghoul Jamel, Medi wafa, Bendayekh afef}

Service de Pneumologie, CHU Habib Bourguiba Medenine /

Service des urgences CHU Habib Bourguiba Medenine

Introduction: Le syndrome d'obésité-hypoventilation (SOH) est défini par l'existence d'une hypoventilation alvéolaire chronique ( $\mathrm{PaO} 2<70 \mathrm{mmHg}, \mathrm{PaCO} 2 \geq 45 \mathrm{mmHg}$ ) chez des patients obèses (IMC $\geq 30 \mathrm{~kg} / \mathrm{m} 2$ ), en l'absence d'autres causes susceptibles d'expliquer les perturbations gazométriques. IL s'agit d'une pathologie sous-diagnostiquée, mais de plus en plus fréquente au vu de l'augmentation de la prévalence de l'obésité.

Patients et méthode: Nous rapportons 28 cas de SOH hospitalisés au service de pneumologie CHU Habib Bourguiba Médenine entre 2015 et 2021.

Résultats: Il s'agissait de 23 femmes et 5 hommes, d'âge moyen de $66 \pm 7$ ans. Des comorbidités cardiovasculaires étaient présentes chez $78,5 \%$ des patients dominés par l'HTA (64,2\%). Tous les patients étaient hospitalisés dans un tableau d'insuffisance respiratoire aigue hypercapnique inaugurale. L'IMC moyen était de 36 $\pm 4 \mathrm{~kg} / \mathrm{m} 2$. La gazométrie artérielle à l'admission démontrait une acidose respiratoire $(\mathrm{pH}$ moyen $=7,28 \pm 5)$ avec hypercapnie $(\mathrm{PaCO} 2$ moyenne $=68 \pm 9 \mathrm{mmHg})$ et hypoxémie sévère $(\mathrm{PaO} 2=$ $52 \pm 11 \mathrm{mmHg}$ ). Un séjour en réanimation était indiqué chez huit malades. Les causes d'exacerbations étaient la surinfection bronchique (42,8\%), décompensation cardiaque gauche $(35,7 \%)$, pneumopathie bactérienne (14,2\%) et l'embolie pulmonaire (7,14\%). A distance de l'épisode aigu, tous les patients avaient bénéficié d'une polygraphie ventilatoire révélant un SAOS sévère chez $46,4 \%$ des patients. La prise en charge était basée sur des mesures diététiques recommandées chez tous les patients, la ventilation nocturne par pression positive continue $(39,2 \%)$ et par ventilation non invasive par BIPAP $(46,4 \%)$. La durée moyenne de suivi était de $30 \pm 8$ mois. L'évolution était favorable chez $75 \%$ des patients. Une mauvaise observance thérapeutique et intolérance à la machine étaient notées dans $17,8 \%$ et $7,1 \%$ des cas, respectivement. Le décès était noté dans deux cas.

Conclusion: Nous insistons à travers ce travail sur le rôle primordial de la ventilation nocturne dans la prise en charge du $\mathrm{SOH}$ en termes d'amélioration clinique et gazométrique, à court et à longue terme.

Conflits d'intérêt: Rien à déclarer 


\section{P187 \\ Embolie Pulmonaire Aiguë Chez les Patients Atteints de COVID-19}

Medi wafa, Cherif Intissar, Kharchoufi Kamel, Bendayekh afef, El Ghoul Jamel

Service de Pneumologie/ service ORL/ service des urgences

Introduction: L'embolie pulmonaire (EP) apparaît aujourd'hui comme l'une des complications les plus dominantes de la pneumonie COVID-19.

But et méthode: Décrire la fréquence de L'EP et ses caractéristiques cliniques à travers une étude rétrospective des cas de COVID-19 hospitalisés au service de pneumologie du CHU Médenine entre septembre 2020 et Mars 2021.

Résultats: Parmi 213 patients hospitalisés pour COVID-19, l'angioscanner thoracique était pratiqué dans 55 cas. Il s'agissait de 15 cas d'EP, avec une moyenne d'âge de $70 \pm 14$ ans et un sexe ratio de 1,14 . Des facteurs de risque de maladie thrombo-embolique étaient notés chez quatre patients: surcharge pondérale dans trois cas et grossesse dans un cas. La dyspnée de repos dominait le tableau clinique initial dans $93 \%$ des cas. Sur le plan radiologique, l'atteinte était sévère dans $40 \%$ des cas, modérée (40\%), étendue (13.3\%) et minime dans $6.7 \%$ des cas. L'EP était segmentaire dans 12 cas et bilatérale dans six cas. Le taux moyen de d-dimères était de $5138 \mu \mathrm{g} / \mathrm{L}$, avec des extrêmes entre 718 et $10000 \mu \mathrm{g} / \mathrm{L}$. La durée moyenne d'hospitalisation était de $14 \pm 10$ jours. Tous les patients avaient reçu une anticoagulation curative à base de HBPM, avec relai par AVK ou anticoagulants oraux directs. L'évolution était marquée par la survenue de complications hémorragiques dans deux cas, le transfert en réanimation et le décès dans trois et deux cas respectivement.

Conclusion: La fréquence de l'EP chez les patients atteints de COVID-19 était élevée (27,2\%). Elle représente aussi la complication la plus redoutable puisqu'elle était associée à une mortalité élevée, estimée à $20 \%$ dans notre série.

Conflits d'intérêt: Rien à déclarer

\section{P188 \\ Impact des Comorbidités Sur L'évolution des Pneumonies à SARS-CoV-2}

\section{A. Allouche, J. Ben Amar, A. Naroura, E. Ben Jemai, O. Tahar, H. Zaibi, H. Aouina}

Service de pneumologie hôpital Charles Nicolle, Service d'immunologie Hôpital Charles Nicolle

Introduction: Les patients ayant une ou plusieurs comorbidités seraient plus exposés à développer des formes sévères de pneumonies à SARS-CoV-2. L'évolution de la maladie étant imprévisible, la présence de comorbidités pourrait induire une augmentation de la morbi-mortalité chez ces patients. L'objectif de notre étude était d'étudier l'impact des comorbidités sur l'évolution au cours de la pneumonie à SARS-CoV-2.
Méthodes: Nous avons mené une étude rétrospective et descriptive ayant inclus 151 patients hospitalisés au service de pneumologie de l'hôpital Charles Nicolle de Tunis pour une pneumonie à SARS-CoV-2 entre septembre 2020 et janvier 2021. Les données des patients ont été recueillies à partir des dossiers médicaux informatisés. L'évolution des patients a été notée. Une évolution péjorative était définie par le transfert en réanimation ou la survenue de décès.

Résultats: Il s'agissait de 91 hommes (H, 60,3\%) et 60 femmes (F, 39,7\%) avec une moyenne d'âge de $63 \pm 13$ ans [29-88]. Parmi notre population, $77 \%$ des patients présentaient au moins une ou plusieurs comorbidités: $41 \%$ une HTA, $12 \%$ une insuffisance coronaire, $11 \%$ une dyslipidémie, $52 \%$ un diabète, $34,6 \%$ une obésité, $4,6 \%$ un asthme, $4 \%$ une BPCO, $2 \%$ un syndrome d'apnée du sommeil, $1,8 \%$ une insuffisance rénale chronique. La saturation pulsée en oxygène moyenne à l'air ambiant chez les patients avec comorbidités était plus basse que chez les patients indemnes de pathologies, sans différence statistiquement significative (84\% vs. $86 \%, p>0,05)$. La durée d'oxygénothérapie à un débit maximal était plus prolongée $(3,84$ vs. 2,5 jours, $p=0,033)$. Trente-cinq $(23,3 \%)$ patients ont été transférés en réanimation. Les patients diabétiques avaient plus de risque de transfert en réanimation avec une différence proche de la significativité $(29,5 \%$ vs. $16,7 \%, \mathrm{p}=0,064)$. Vingt patients étaient décédés (13,2\%).

Conclusion: Les comorbidités prédisposeraient à un risque accru de pneumonie COVID-19 sévère avec une mauvaise évolution clinique. Mieux connaitre ces facteurs de risque permettrait une prise en charge précoce et optimale des patients.

Conflits d'intérêt: Rien à déclarer

\section{P189 \\ Corrélation Entre la Sévérité Clinique et le Profil Sérologique dans les Pneumonies à SARS Cov2}

J. Ben Amar, A. Naroura, A. Allouche, E. Ben Jemai, O. Tahar, I. Sfar*, Y. Gorgi*, H. Zaibi, H. Aouina

Service de pneumologie hôpital Charles Nicolle, Service d'immunologie Hôpital Charles Nicolle

Introduction: La maladie à coronavirus 2019 est causée par le coronavirus 2 du syndrome respiratoire aigu sévère (SARS-CoV-2) qui est une maladie émergente responsable d'une pandémie depuis décembre 2019. Beaucoup d'études se sont intéressées aux facteurs de risques et aux facteurs pronostics. Cependant peu d'études se sont intéressées à étudier le taux des anticorps et leurs cinétiques en fonction de la sévérité clinique.

L'objectif de notre étude était d'étudier le taux des anticorps à type d'IgG et IgM dans l'infection à SARS-COV2 et leurs cinétiques selon la gravité clinique.

Méthode: Il s'agissait d'une étude transversale réalisée au centre hospitalier universitaire Charles Nicolle de Tunis entre octobre 2020 et Janvier 2021 ayant inclus 50 patients hospitalisés pour pneumopathie à SARS-COV2. Les données cliniques, anthropométriques des patients ont été recueillies. Tous les patients ont eu un bilan sanguin (bilan rénal, hépatique, NFS, CRP, D-Dimères, 
Troponines) et une sérologie Covid 19 à J1, J7 et J14 de l'hospitalisation. Le diagnostic a été retenu soit par une PCR positive ou par scanner thoracique évocateur.

Résultats: L'âge moyen de nos patients était de 61,25 $\pm 12,56$ ans [31-85 ans]. Le sexe ratio était de 1,63 avec 31 hommes versus 19 femmes. La technique Roche était utilisée chez 33 patients, tandis que les techniques Beckman Coulter et Medibio étaient utilisées respectivement chez 12 et 05 patients. Le nombre de sérologies positives à J01 étaient de 36 versus 14 négatives. À J07, 45 patients avaient une sérologie positive versus 5 patients avaient une sérologie négative et à J14 un seul patient gardait une sérologie négative.

Les patients ayant nécessité $10 \mathrm{~L} / \mathrm{min}$ d'O2 ou plus ont été considérés comme ayant des formes graves (31,2\% des patients). Aucune corrélation n'a été retrouvée entre le taux des anticorps, leurs cinétiques et l'évolution vers les formes graves et le pronostic de la maladie. On n'a pas trouvé également de corrélation entre le taux des lymphocytes et le taux des immunoglobulines. Par contre plus les patients avaient un taux lymphocyte bas, plus la forme était sévère $(\mathrm{p}=0,019)$.

Conclusion: Conformément aux données de la littérature les formes graves de la Covid 19 étaient corrélées au degré de la lymphopénie. Néanmoins, notre étude a montré qu'au aucune corrélation n'a été retrouvée entrele taux des anticorps etl'évolution clinique vers la forme grave.

Conflits d'intérêt: Rien à déclarer

\section{P190 \\ Profil Épidémiologique, Clinique, Paraclinique et Évolutif des Pneumonies Aigues Communautaires Hospitalisées au Service de Pneumologie du Chu Mohamed VI de Marrakech}

\section{S. Kechnaoui, S. Aitbatahar, L. Amro}

Service de pneumologie. Labo. PCIM, UCA, Marrakech, Maroc

Introduction: La pneumonie aiguë communautaire (PAC) représente une forme à risque des infections respiratoires basses communautaires qui pose un problème de prise en charge. But de travail: Discuter les particularités épidémiologiques, radiocliniques et le profil bactériologique des PAC colligées dans le service de pneumologie du CHU Mohamed VI de Marrakech.

Matériels et méthodes: Une étude rétrospective réalisée sur une période de 3 ans entre janvier 2018 et mars 2021.

Résultats: soixante-treize cas de PAC ont été colligés. Il s'agit de 42 femmes et 31 hommes. La moyenne d'âge des patients était de 60 ans \pm 17.68 , dont $44 \%$ âgés de plus de 65 ans. Une comorbidité a été notée chez 38 cas, dominée par le diabète, l'HTA et l'insuffisance rénale. Un antécédent respiratoire a été noté chez 26 cas, dominé par une tuberculose pulmonaire traitée, un asthme et une pneumonie aigue. Un séjour initial en réanimation a été noté chez 21 cas. Le délai d'hospitalisation était en moyenne de 12 jours, avec un tableau clinique dominé par les syndromes bronchiques et infectieux. L'examen pleuropulmonaire a trouvé principalement des signes en foyer. La radiographie thoracique a objectivé un foyer alvéolaire chez 63 patients, bilatéral chez 25 cas. La documentation microbiologique a été obtenue dans $53 \%$ des cas, montrant essentiellement une infection à BGN, à pneumocoque et à staphylocoque à coagulase négative. L'antibiothérapie était initialement probabiliste puis adapté en fonction de l'antibiogramme et l'évolution clinico-biologique du patient, faite principalement d'une bithérapie (67\%). La durée moyenne de traitement était de 10 jours. L'évolution était favorable dans la majorité des cas (97\%).

Conclusion: Les PAC nécessitent un diagnostic et une prise en charge rapide et adéquate basée le plus souvent sur une antibiothérapie probabiliste.

Conflits d'intérêt: Rien à déclarer

\section{P191 \\ Facteurs Influençant la Durée D'hospitalisation des Patients Pris en Charge Pour une Infection à SARS-CoV2}

\section{A. Hedhli, M. Chaabane, M. Mjid, M. Klila, Y. Ouahchi, S. Cheikhrouhou, S. Merai, S. Toujani, B. Dhahri}

Université de Tunis El Manar, Faculté de médecine de Tunis, service de pneumologie hôpital la Rabta, LR18SP02, Tunis Tunisie

Introduction: Le COVID-19 est une maladie virale apparue en Chine et menant à une pandémie dès mars 2020. La prise en charge des patients atteints du SARS-CoV2 peut être ambulatoire ou dans un milieu hospitalier. Plusieurs facteurs peuvent conditionner la durée d'hospitalisation.

Objectif: Etudier les facteurs qui peuvent influencer la durée d'hospitalisation des patients hospitalisés pour une pneumopathie à SARS-CoV2 sévère.

Méthodes: Il s'agissait d'une étude rétrospective, menée au Service de Pneumologie de l'Hôpital La Rabta, sur une période étendue sur 4 mois (de septembre 2020 à janvier 2021). Les patients ayant été admis dans notre service pour pneumopathie à SARSCoV2 sévère, selon la définition de l'OMS, ont été inclus. Les données cliniques et biologiques ont été recueillies des dossiers médicaux.

Résultats: Il s'agissait de 150 patients, âgés en moyenne de 59,8 ans (17 à 90 ans) avec un sexe ratio à 0,85 . Cent vingt-deux patients présentaient au moins une comorbidité (81,3\%). La durée moyenne d'hospitalisation était de 7,75 jours. La durée d'hospitalisation était significativement plus importante chez les sujets âgés de plus de 65 ans $(8,3$ jours vs 6,9 jours; $p=0,01)$ et chez les obèses (IMC $>30 \mathrm{Kg}$ / m2) $(10,1$ vs 6,8 jours; $p<0,001)$. Sur le plan clinico-biologique, les patients ayant nécessité un débit d'oxygène $>101 /$ min et ceux ayant une lymphopénie à l'admission ont nécessité une durée d'hospitalisation plus prolongée ( $\mathrm{p}=0,004$ et $\mathrm{p}=0,03$ respectivement). Une corrélation significative a été retrouvée entre la dureé d'hospitalisation et l'âge $(\mathrm{p}=0,02)$, les besoins en oxygène $(\mathrm{p}=0,01)$, l'IMC ( $\mathrm{p}=0,03)$, le taux de lymphocytes $(\mathrm{p}=0,01)$ et l'étendue scannographique des lésions parenchymateuses $(\mathrm{p}=0,02)$ à l'admission. Aucune corrélation n'a été retrouvée avec les taux de CRP ou des D-Dimères. 
Conclusion: En concordance avec les données de la littérature, notre étude a montré que la durée d'hospitalisation des patients atteints de pneumopathie à SARS-CoV2 sévère était influencée par l'âge, l'obésité, la sévérité du tableau clinique initial, la lymphopénie et l'étendue de l'atteinte parenchymateuse au scanner thoracique.

Conflits d'intérêt: Rien à déclarer

\section{P192}

\section{Test de Marche de 6 Minutes Chez les Sujets BPCO}

\section{Bougadoum, S. Ait Batahar, L. Amro}

Service de pneumologie, Hôpital Arrazi, CHU de Marrakech, Maroc

Introduction: La BPCO est définie actuellement comme une maladie générale à point de départ respiratoire, de nombreuses comorbidités viennent compliquer son évolution naturelle et aggrave son pronostic. L'intolérance à l'effort principale plainte du patient, est le résultat d'une double composante: respiratoire et musculaire périphérique. Sur le plan pratique, cette incapacité à l'exercice est évaluée par le test de marche de 6 minutes qui aurait une valeur prédictive de mortalité chez les sujets BPCO.

Méthode: Il s'agit d'une étude prospective et analytique dont l'objectif est d'évaluer la tolérance à l'effort chez nos sujets atteints de BPCO par le test de marche de 6 minutes sur une période de suivi de 5 mois. Le T6 M est réalisé chez tous les patients BPCO en état stable et en absence de contre-indications (angor instable, infarctus du myocarde récent).

Résultats: Notre cohorte est composée de 40 patients BPCO, d'âge moyen de 63 ans avec une prédominance masculine nette (97,5\%). Le VEMS post bronchodilatateur moyen des patients est de 1,4 L. L'indice de masse corporelle (IMC) des BPCO est en moyenne de $24,1 \mathrm{~kg} / \mathrm{m} 2$. La distance de marche moyenne est de $403 \mathrm{~m}$ avec des extrêmes de $150-660 \mathrm{~m}$. 42\% ont parcouru une distance de plus de $350 \mathrm{~m}$ et $13 \%$ avait un T6M moins de $100 \mathrm{~m}$. Une désaturation a été retrouvée dans 7,5\%. Les patients au Gold II ont parcouru en moyenne $378 \mathrm{~m}, 290 \mathrm{~m}$ pour le Gold 3, et 170 $\mathrm{m}$ pour le Gold 4. Le T6M est étroitement corrélé au VEMS et à l'échelle de la dyspnée (mMRC).

Conclusion: Le TM6 constitue un outil précieux, objectif et reproductible dans l'évaluation de la sévérité de la BPCO et dans l'optimisation de sa prise en charge thérapeutique.

Conflits d'intérêt: Rien à déclarer

\section{P193 Chirurgie des Corps Étrangers Chez L'enfant: à Propos de 13 Cas}

M. Abdennadher ${ }^{1}$, E. Khlas ${ }^{1}$, I. Bouacida ${ }^{1}$, H. Zribi ${ }^{1}$, S. Zairi ${ }^{1}$,
A. Abdelkebir ${ }^{1}$, H. Neji ${ }^{3}$, M. Mlika ${ }^{5}$, S. Ouerghi ${ }^{5}$, A. Marghli ${ }^{1}$

Service de Chirurgie thoracique et cardiovasculaire de l'hopital A. Mami de l'Ariana

Introduction: L'inhalation d'un corps étranger chez l'enfant représente une situation d'urgence pouvant mettre en danger la vie de l'enfant pour laquelle une intervention rapide est impérative. L'extraction endoscopique reste le traitement de première intention mais parfois elle peut s'avérer dans certains cas non réalisable, $\mathrm{du}$ fait de la localisation ou la nature du corps. Ainsi la chirurgie reste la seule alternative thérapeutique.

Méthode: Il s'agit d'une étude rétrospective sur une période de 5 ans incluant 14 enfants opérés pour un corps étranger intra-bronchique au service de chirurgie thoracique et cardiovasculaire de l'hôpital A. Mami de l'Ariana. Ont été exclus les patients d'âge supérieur à 14 ans et dont l'intervalle entre l'inhalation et la chirurgie excède 30 jours.

Résultats: L'âge moyen des patients opérés était de 10,7 ans avec une nette prédominance féminine à $77 \%$. La symptomatologie était dominée par la toux dans $84 \%$ des cas suivie par la gêne respiratoire, un seul cas de crachats hémoptoïques a été rapporté. Le diagnostic de corps étranger a été rapporté dans la majorité des cas, soit sur une radiographie du thorax Une tentative d'extraction endoscopique a été réalisée chez la totalité des patients, la localisation des corps étrangers la plus retrouvée était au niveau de la bronche souche gauche avec $46 \%$ des cas, le délai entre l'inhalation et la chirurgie était en moyenne de 14,15 jours. $76 \%$ des patients ont eu une bronchotomie, $15 \%$ une pneumotomie et $7,6 \%$ une lobectomie, la voie d'abord était une thoracotomie postéro-latérale dans $92 \%$ des cas. La moyenne de drainage était de 2.76 jours et les suites étaient simples dans chez tous les patients.

Conclusion: La chirurgie des corps étranger possède de bons résultats, elle doit néanmoins être précoce devant le risque de complications infectieuses qui rendent le geste chirurgical difficile vu les phénomènes hyper-inflammatoires se créant au niveau du parenchyme pulmonaire

Conflits d'intérêt: Rien à déclarer 


\section{P194 \\ Pandémie COVID-19: Profil Épidémiologique, Clinique et Évolutif des Patients Hospitalisés au Service de Pneumologie à L'hôpital Mohamed Taher Maamouri Nabeul-Tunisie}

Gader Nada ${ }^{1}$, ABID Narjes ${ }^{1}$, Kalboussi Slim ${ }^{1}$, Zineddine Hamza', Loukil Manel', Chaabane Imen ${ }^{2}$, Ben Ali Mechaal ${ }^{3}$, Ghrairi Hedia ${ }^{1}$

${ }^{1}$ Service de pneumologie- CHU Mohammed Taher Maamouri Nabeul, ${ }^{2}$ Service de médecine interne- CHU Mohammed Taher Maamouri Nabeul, ${ }^{3}$ Service de réanimation médicale- $\mathrm{CHU}$ Mohammed Taher Maamouri Nabeul

Introduction: La pandémie COVID-19 s'est déclarée à Wuhan en décembre 2019. En mars 2020, cette pandémie a touché notre pays et seulement quelques dizaines de cas ont été déclarées. Ce n'est qu'en septembre 2020, que cette épidémie a frappé notre pays de plein fouet. On se propose, à travers ce travail d'étudier les caractéristiques épidémiologiques, cliniques et évolutives des patients hospitalisés pour une infection SARS-COV2 pendant cette vague épidémique.

Méthode: Il s'agit d'une étude prospective menée auprès des patients hospitalisés pour une infection au SARS-CoV-2, confirmée par RT-PCR, durant la période allant de septembre 2020 à Mars 2021 au service de pneumologie à l'hôpital MTM, Nabeul.

Résultats: Deux cent soixante-dix-sept patients ont été inclus. Cinquante-huit pour cent des patients étaient des hommes avec un sexe-ratio de 1,38. L'âge médian était de 64 ans. La plupart des patients (80\%) étaient des non-fumeurs. Les comorbidités les plus constatées étaient l'HTA (54\%) et le diabète (43\%). Les antécédents respiratoires n'étaient présents que dans $16 \%$ des cas. Cent vingtdeux patients (44\%) étaient obèses. La quasi-totalité des patients $(99,3 \%)$ étaient symptomatiques, avec un délai consultation moyen de 9 jours [1-39]. Les symptômes les plus fréquents étaient une dyspnée $(76 \%)$, une toux sèche $(64 \%)$, une fièvre $(46 \%)$ et une asthénie (43\%). Aucun cas de manifestations dermatologiques n'a été constaté. A l'admission, $82 \%$ des patients avaient une $\mathrm{SpO} 2$ inférieure à $94 \%$ et $43,5 \%$ ont nécessité un débit d'oxygène d'au moins de $6 \mathrm{l} / \mathrm{mn}$. Une fièvre était notée dans $18 \%$ des cas. L'auscultation pulmonaire avait trouvé des râles crépitants dans $58 \%$ des cas. La durée d'hospitalisation était en moyenne de 9 jours [1-53]. La plupart des patients (75\%) ont été déclarés guéris et mis sortants sans oxygénothérapie à domicile, alors que 15\% ont été transférés en réanimation et $12,6 \%$ sont décédés.

Conclusion: Une meilleure prise en charge de l'infection à SARS-COV2 passe par une meilleure connaissance de ses présentations cliniques et de ses modalités évolutives

Conflits d'intérêt: Rien à déclarer

\section{P195 \\ Manifestations et Complications Extra-Pulmonaires de la Pneumonie COVID-19}

Gader Nada ${ }^{1}$, ABID Narjes ${ }^{1}$, Kalboussi Slim ${ }^{1}$, Zineddine Hamza ${ }^{1}$, Loukil Manel', Chaabane Imen ${ }^{2}$, Ben Ali Mechaal ${ }^{3}$, Ghrairi Hedia ${ }^{1}$

${ }^{1}$ Service de pneumologie- CHU Mohammed Taher Maamouri Nabeul, ${ }^{2}$ Service de médecine interne- CHU Mohammed Taher Maamouri Nabeul, ${ }^{3}$ Service de réanimation médicale- $\mathrm{CHU}$

Mohammed Taher Maamouri Nabeul

Introduction: Les manifestations cliniques extra pulmonaires de l'infection à SARS-COV2 sont polymorphes et ses complications sont multiples pouvant toucher les différents systèmes notamment cardiovasculaire, neurologique mais aussi digestif et rénal. Le but de ce travail est de décrire les principales manifestations et complications extra-pulmonaires chez les patients hospitalisés pour une pneumonie COVID-19.

Méthode: Il s'agit d'une étude prospective réalisée dans le service de pneumologie au CHU Mohamed Taher Maamouri, Nabeul incluant 277 patients hospitalisés pour infection à SARSCOV2 entre septembre 2020 et Mars 2021.

Résultats: Des symptômes digestifs étaient présents chez 61 patients $(22 \%)$ à type de nausées et vomissements $(n=22 ; 8 \%)$, douleurs abdominales $(n=16 ; 5.8 \%)$ et de diarrhée $(n=23 ; 8.4 \%)$. Onze patients ont présenté une agueusie et 15 patients une anosmie. Les manifestations neuro-psychiatriques décrites étaient une confusion chez 4 patients $(1,5 \%)$ et une crise convulsive chez une patiente ancienne épileptique avec une IRM cérébrale normale. Les complications notées, pendant le séjour hospitalier, étaient notamment cardiovasculaires (17.6\%): 11 cas d'embolie pulmonaire, 12 cas de syndrome coronarien aigu, 20 cas de poussée d'insuffisance cardiaque, des troubles du rythme cardiaque dans 5 cas et un seul cas de myocardite. Une insuffisance rénale fonctionnelle est survenue chez 47 patients (17\%) alors que l'insuffisance rénale organique a été diagnostiquée dans 8 cas avec passage à l'hémodialyse dans 4 cas. Les complications digestives étaient à type de cytolyse hépatique dans 49 cas avec des chiffres d'enzymes hépatiques allant jusqu'à 15 fois la normale et de pancréatite dans un seul cas. Le déséquilibre de diabète est survenu dans $29,7 \%$ cas ( 82 patients), avec nécessité de passage à l'insuline dans $19 \%$ des cas. 
Conclusion: Il est essentiel de prendre conscience del'incidence de ces complications extra pulmonaire de la pneumonie COVID-19 afin de les diagnostiquer et de les traiter à temps.

Conflits d'intérêt: Rien à déclarer

\section{P196}

\section{Pneumonie COVID19: Les aspects Radiologiques Atypiques}

Kalboussi Slim ${ }^{1}$, ABID Narjes1, Gader Nada ${ }^{1}$, Loukil Manel ${ }^{1}$, Chaabane Imen ${ }^{2}$, Bouzaidi Khaled ${ }^{3}$, Ghrairi Hedia'

${ }^{1}$ Service de pneumologie- CHU Mohammed Taher Maamouri Nabeul, ${ }^{2}$ Service de médecine interne- CHU Mohammed Taher Maamouri Nabeul, ${ }^{3}$ Service d'imagerie médicale- CHU Mohammed Taher Maamouri Nabeul

Introduction: L'infection par le SARS COV2 est une maladie infectieuse caractérisée par une diversité clinique, biologique et radiologique. L'apport de l'imagerie dans ce contexte est fondamental pour orienter le diagnostic, évaluer le degré d'atteinte pulmonaire mais aussi pour déceler les différentes complications. Cependant, plusieurs présentations radiologiques atypiques peuvent faire errer le diagnostic. Le but de cette étude est de déterminer les différents aspects scannographiques atypiques observés chez les patients atteints d'une infection SARS COV2.

Méthode: Il s'agit d'une étude prospective menée auprès des patients hospitalisés dans le service de pneumologie du CHU Mohamed Taher Maamouri, Nabeul, pour une infection au SARSCoV-2 durant la période allant de Septembre 2020 à Mars 2021.

Résultat: Cette étude a inclus 277 patients dont $58 \%$ étaient des hommes. Le sexe ratio était de 1,38. L'âge moyen était de 64 ans. Le diagnostic a été confirmé par une PCR dans $88 \%$ des cas $(n=246)$. Une TDM thoracique a été réalisé chez 63\% $(n=177)$ de nos patients. L'aspect typique de verre dépoli a été observé dans $37,7 \%$ d'entre eux. Les condensations parenchymateuses ont été rapporté dans $11,2 \%(\mathrm{n}=16)$ des cas et l'association des deux était présentes dans $45 \%$ des cas.

L'imagerie a aussi mis en évidence une pleurésie dans $12,4 \%$ des cas et l'aspect de crazy paving dans 5 cas. L'atteinte médiastinales étaient à type d'adénopathies médiastinales retrouvées chez 6 patients $(3,6 \%)$ et de pneumomédiastin (2 patients).

Conclusion: L'imagerie médicale a un rôle important dans la prise en charge des patients atteint d'une infection SARS COV2 d'où l'importance de connaitre les différents aspects radiologiques possibles.

Conflits d'intérêt: Rien à déclarer

\section{P197 \\ Les Comorbidités Cardio Vasculaire, et \\ Métaboliques des Malades Hospitalisés en Unité de Soins Intensifs Covid 19}

\author{
N. Ouakil, S. Aitbatahar, L. Amro \\ Pneumologie, CHU mohamed VI Marrakech
}

Introduction: La maladie à coronavirus 2019 (COVID-19) est une infection respiratoire aiguë potentiellement grave. Plusieurs facteurs influencent la sévérité de la maladie dont les maladies cardiovasculaires et métaboliques. But de travail: évaluer le retentissement des maladies cardiovasculaires et métabolique sur la maladie à coronavirus chez les patients covid.

Matériels et méthodes: C'est une étude rétrospective incluant les cas d'HTA, cardiopathie et diabète en unité de soins intensifs covid 19 marrakech entre aout 2020 et décembre 2020.

Résultats: on a colligé 122 cas dont $52.4 \%$ présentent une comorbidité cardio vasculaire et métabolique: diabète: $38.5 \%$, HTA: $32.7 \%$, cardiopathie $7.3 \%$. L'examen clinique des patients avec comorbidité trouve $\mathrm{SaO} 2<90: 46.8 \%$, entre 90 et 95: $42,1 \%$ et $>95: 10.9 \%$. Fréquence respiratoire $>24: 56.2 \%$ et $<24$ : 43.7\%, FC>90: entre 60 et 90: 30 cas $(45.4 \%)$ et $<60: 4$ cas(6\%). sur la TDM l'atteinte était légère: $20.3 \%$ modérée: $31.2 \%$, sévère: $37.5 \%$, et critique: $10.9 \%$, aspect en verre dépoli: $92.1 \%$, condensation alvéolaire bilatérale: $45.3 \%$ et aspect en mosaïque: $37.5 \%$. Sur le plan biologique: hyperleucocytose: 39\%, lymphopénie: 39\%, thrombopénie: $6.2 \%$, hyperglycémie: $34.3 \%$, insuffisance rénale: $15.6 \%$ augmentation des D-dimères: $21.8 \%$. L'acidocétose diabétique: $17.1 \%$. L'oxygénothérapie: entre 2 et $6 \mathrm{l} / \mathrm{min}$ : $34.3 \%$, entre 6 et 10 l/min: $40.6 \%$ et entre 10 et 15: 25\%. L'évolution a été marqué par: sortie sans oxygénothérapie: $15.3 \%$ avec oxygénothérapie: $31.2 \%$, un transfert en réanimation: $25 \%$, décès: $1.5 \%$.

Conclusion: Les maladies cardio-vasculaires et métaboliques jouent un rôle essentiel dans la gravité de la maladie du COVID-19.

Conflits d'intérêt: Rien à déclarer

\section{P198 \\ Étude Comparative Entre le Profil du Pneumothorax Chez le Sujet Jeune et le Sujet Âgé}

N. Ouakil, S. Aitbatahar, L. Amro

pneumologie CHU mohamed VI Marrakech

Introduction: Le pneumothorax correspond à la survenue d'un épanchement aérien dans la cavité pleurale.

Méthode: Nous rapportons une étude rétrospective entre 2018 et 2020 comparant le profil du pneumothorax chez 122 patients répartis en 2 groupes: groupe $\mathrm{A}(\mathrm{n}=64)$ âgé de moins de 40 ans avec une moyenne d'âge de 30 ans, et groupe $B(n=58)$, âgé de plus de 60 ans avec une moyenne d'âge de ans.

Résultats: La prédominance du sexe masculin est notée dans les 2 groupes: groupe A 87.5\% et groupe B 89.5\%. Le tabagisme est noté dans $60.3 \%$ du groupe A vs $89.5 \%$ du groupe B. Les signes 
cliniques révélateurs sont dominés par la douleur thoracique, la dyspnée et la toux chez tous les patients. La radiographie thoracique a montré, un pneumothorax total dans $92.1 \%$ des cas du groupe A vs $89.7 \%$ des cas du groupe B, partiel dans $7.9 \%$ des cas du groupe A vs $10.3 \%$ des cas du groupe B, et bilatéral dans $4.7 \%$ du groupe A vs $1.7 \%$ du groupe B. L'étiologie qui prédomine dans les deux groupes l'emphysème pulmonaire: groupe A $46.9 \%$ et groupe B: $75.9 \%$. Les autres origines dans le groupe A sont: la rupture de blebs dans $25 \%$ des cas, la tuberculose pulmonaire dans 17.2\% des cas, les séquelles de tuberculose dans 6.3\% des cas, silicose dans 2 cas et idiopathique dans un cas. Dans le groupe B, les étiologies sont: les sequelles de tuberculose dans $6.9 \%$ des cas, carcinome bronchique et PID dans $3.4 \%$ chacun, tuberculose pulmonaire, la miliaire tuberculeuse bronchopneumonie, pneumoconiose et sarcoidose dans $1.7 \%$ chacun. Le traitement est basé essentiellement sur le drainage thoracique dans les 2 groupes avec une durée moyenne de bullage de cinq jours. L'évolution était favorable dans $78.1 \%$ du groupe A vs $62.1 \%$ des cas du groupe B.

Conclusion: Nous soulignons par ce travail; que le pneumothorax chez le sujet jeune et sujet âgé est souvent secondaire à une rupture d'une bulle d'emphyséme d'évolution généralement favorable.

Conflits d'intérêt: Rien à déclarer

\section{P199 \\ Les Manifestations Digestives Chez les Malades Hospitalisés Pour une Infection SARS-COV-2}

N. Ouakil, H. Benaziz, S. Aitbatahar, L. Amro

pneumologie CHU mohamed VI Marrakech

Introduction: La Covid-19 est une maladie respiratoire dont les symptômes peuvent faire penser à la grippe avec des complications pulmonaires. Mais d'autres symptômes, plus atypiques existent aussi, comme, la perte d'odorat ou encore les troubles digestifs. But de travail: Le but de notre travail était d'évaluer la prévalence, le spectre, la gravité et l'importance des manifestations digestives chez les patients hospitalisés pour COVID-19.

Matériels et méthodes: C'est une étude rétrospective chez les malades hospitalisés en unité de soins intensifs covid 19 marrakech entre aout 2020 et decembre 2020.

Résultats: on a colligé 122 cas; Il s'agit de 23 hommes (59.5\%) et 8 femmes (40.5\%) dont l'âge moyen était de 50 ans: 73 ans chez les hommes et 50 ans chez les femmes. Dans l'ensemble, 56.55\% des patients ont présenté au moins un symptôme gastro-intestinal à un moment quelconque de leur maladie, le plus souvent des diarrhées $(24.5 \%)$, des nausées (16.7\%), des vomissements $(15.3 \%)$ et des douleurs abdominales (11\%). Dans 63\% des cas, les symptômes gastro-intestinaux ont été jugés légers. $35.24 \%$ des malades ont une élévation des phosphatases alcalines, $15.57 \%$ ont une augmentation des GGT et $35.24 \%$ ont développé un taux anormal des ALAT, ASAT ou de bilirubine totale; ceux-ci étaient élevés à moins de 5 fois la limite supérieure de la normale dans $76.5 \%$ des cas.

Conclusion: Parmi les patients hospitalisés avec COVID-19, les symptômes gastro-intestinaux et les anomalies du bilan hépatique étaient courants mais la majorité d'entre eux étaient légers et leur présence n'était pas associée à une évolution clinique plus sévère.

Conflits d'intérêt: Rien à déclarer

\section{P200 \\ Facteurs Prédictifs de la Durée du Séjour Hospitalier Pour une Pneumopathie Aigue Communautaire}

\author{
H. Mrassi, N. Belloumi, I. Bachouch, I. Nouira, N. Khezami, \\ C. Habouria, F. Chermiti, S. Fenniche
}

Service de pneumologie Pavillon 4 - CHU A. Mami Ariana

Introduction: La pneumonie aigue communautaire (PAC) constitue un problème de santé publique de par sa fréquence, son coût et sa morbi-mortalité.

Buts: Rechercher les facteurs prédictifs de la prolongation du séjour hospitalier des PAC sévères.

Méthode: Etude rétrospective menée sur les dossiers de patients (pts) hospitalisés pour PAC sévère au Pavillon 4 du CHU A. Mami [2016 - 2019]. L’analyse statistique était réalisée via SPSS 25.0.

Résultats: Cent vingt-six pts de sexe masculin étaient inclus. L'âge moyen était de 61 ans [15-92]. Soixante-dix-huit pourcent des pts étaient tabagiques à une moyenne de 55 paquets-années. Les comorbidités les plus fréquentes étaient: la BPCO (36\%), le diabète $(27 \%)$ et l'HTA (19\%). Le score de Fine moyen calculé à l'admission était de $85 \pm 33$. L'enquête bactériologique avait isolé un germe chez $11.1 \%$ des cas. La durée moyenne d'hospitalisation (D) était de $9 \pm 5$ jours. $D$ était de moins de 7 jours dans $55 \%$ des cas. La durée $\mathrm{D}$ n'était corrélée ni à l'âge, ni à l'intoxication tabagique, ni au germe isolé, ni au score de Fine. La durée D était plus prolongée en présence de diabète $(\mathrm{p}=0,04)$. Par ailleurs, $\mathrm{D}$ était corrélée à l'étendue radiologique de la pneumonie $(\mathrm{p}=0,04)$ et à la présence d'une pleurésie associée $(\mathrm{p}=0,03)$. La modification de l'antibiothérapie pendant l'hospitalisation était associée à une $\mathrm{D}$ plus longue (17 vs 8 jours, $\mathrm{p}=0,001$ ).

Conclusions: Selon nos résultats, la présence de diabète, l'étendue radiologique, la pleurésie associée et la résistance à l'antibiothérapie de le intention sont prédictifs d'un séjour hospitalier prolongé.

Conflits d'intérêt: Rien à déclarer 


\section{P201 \\ Valeur Pronostique de la Qualité de Vie au Cours du Cancer Pulmonaire Non à Petites Cellules Stade IV}

\section{N. Belloumi, H. Mrassi, I. Bachouch, N. Khezami, I. Nouira, C. Habouria, F. Chermiti, S. Fenniche}

Service de pneumologie Pavillon 4 - CHU A. Mami Ariana

Introduction: Le pronostic du cancer pulmonaire non à petites cellules (CNPC) aux stades avancés, est sujet de plusieurs essais cliniques. L'impact pronostique des différents indicateurs de la qualité de vie n'est pas clairement élucidé.

Buts: Evaluer la corrélation entre les indicateurs de la qualité de vie avec la survie globale des patients (pts) au CNPC stade IV.

Méthode: Etude prospective menée sur les patients suivis et traités par chimiothérapie (CT) pour CNPC stade IV au pavillon 4 de l'hôpital A. Mami. Les pts porteurs d'une co-morbidité chronique déséquilibrée affectant la qualité de vie étaient exclus. Le questionnaire QLQ-C30 (version dialectale tunisienne) était utilisé pour évaluer la qualité de vie des patients après fin de la chimiothérapie.

Résultats: Soixante et onze pts de sexe masculin étaient inclus. L'âge moyen était de 62 ans [42-85]. Tous les pts étaient tabagiques. La BPCO était la comorbidité la plus fréquente $(n=28,39 \%)$. Soixante-deux pourcent pts $(n=44)$ avaient un PS conservé. Le type histologique le plus fréquent était l'adénocarcinome $(n=41,58 \%)$. La répartition selon les stades était: IV A (27\%), IV B (34\%) et IV C (39\%). Le score moyen du coefficient des symptômes était de 26,5 et celui des performances de 64 . Vingt-six pts (37\%) avaient une bonne réponse après une CT de 1e ligne. Leur SSP moyenne était de 194 jours [60-550]. La survie globale moyenne (SG) était de 317 jours [292 - 377]. La survie globale était corrélée au coefficient de la vie globale ( $\mathrm{p}=0,0001, \beta=0,547)$ et au coefficient des performances $(p=0,0001, \beta=0,617)$. La survie globale inversement corrélée au coefficient des symptômes ( $\mathrm{p}=0,0001, \beta=-0,538)$.

Conclusions: La dégradation de la qualité de vie, chez les pts sous CT pour CNPC stade IV, est corrélée à un mauvais pronostic.

Conflits d'intérêt: Rien à déclarer

P202

\section{Similitude et Différence Entre L'infection Tuberculeuse et le COVID 19: Étude Comparative Réalisée à la Région d'AGADIR}

\author{
Oussama Halloumi, Soumya Lotfi, Houda Moubachir, \\ Hind Serhane
}

Faculté de Médecine et de Pharmacie d'Agadir (FMPA), Université lbn Zohr (UIZ), Agadir, Maroc

Introduction: Le COVID-19 est une maladie virale aiguë, tandis que la tuberculose est une maladie bactérienne chronique; ces deux pathologies ont des symptômes en commun, ainsi que plusieurs facteurs de risques (le diabète, l'immunodépression, etc...). Le but de ce travail est de rapporter les différences et similitudes entre la tuberculose et le COVID-19, leurs diagnostics, leurs caractéristiques épidémiologiques et cliniques et leurs implications pour la santé publique.

Méthode: Etude rétrospective et comparative entre deux groupes; (G1: groupe de patient tuberculeux et G2: groupe de patient COVID 19), colligés au service de COVID de l'hôpital HASAN II et au CDTMR, réalisée à la région d'AGADIR entre la période de MARS 2020 - FEVRIER 2021.

Résultats: Sur un ensemble de 210 cas, le G1 représente 105 cas TPM+, contre 105 cas de COVID19 pour le G2. On notait une prédominance masculine pour les deux groupes. La moyenne d'âge a été respectivement de (36 ans vs 61 ans). Les comorbidités ont été plus marquées dans le groupe G2 dans $66,6 \%$ des cas: HTA 17 cas $(16,1 \%)$, diabète sucré 43 cas $(40,9 \%)$, cardiopathie 8 cas (7,6\%). Sur le plan clinique: Les symptomatologies étaient dominés par la toux dans les 2 groupes (96,19\% vs 76,19\%), par l'asthénie $(72,38 \%)$, La dyspnée est plus marquée dans le groupe G2 dans $83,80 \%$ contre $19,04 \%$ dans le $\mathrm{G} 1$, les douleurs thoracique $(30,47 \%$ contre $54,28 \%$ ), les signes digestifs était plus présents dans le groupe $\mathrm{G} 2$. Le bilan biologique a révélé une lymphopénie dans $42,3 \%$ des cas dans le groupe G1 contre $65,21 \%$ dans le G2.

Conclusion: Le COVID-19 et la tuberculose ont tous deux la capacité de stresser les systèmes de santé, ce sont des maladies transmissibles aéroportées. Ils nécessitent une sensibilisation et une coopération du public pour permettre une efficace prévention.

Conflits d'intérêt: Rien à déclarer

\section{P203 \\ Evaluation de L'intérêt de la Stratégie de \\ Prescription de L'héparine À Bas Poids Moléculaire \\ dans la Prise en Charge des Patients Admis à \\ L'unité de Soins Intensifs COVID-19 (USI-COVID19) \\ Pneumologique au CHU Mohammed VI, Marrakech-Maroc}

P. P. Koumeka*1 , C. Rachid, S. Ait Batahar ${ }^{1}$, L. AMRO

Pneumologie, CHU Mohammed VI, Marrakech-Maroc

Introduction: Les troubles de coagulation sont associées à une mortalité élevée par COVID-19.

Objectif: évaluer l'intérêt et la stratégie de prescription de l'héparine à bas poids moléculaire (HBPM) dans la prise en charge des patients admis à l'USI COVID-19 Pneumologique.

Méthode d'étude: Il s'agit d'une étude descriptive et analytique, allant d'octobre 2020 à décembre 2020, menée à l'USI COVID-19 Pneumologique. Tous les patients admis pendant la période d'étude étaient inclus, traités tous à base d'héparine à bas poids moléculaire en prophylaxie (mono dose de $4000 \mathrm{UI}$ ) ou en en traitement curatif (double dose de 6000 UI). Le devenir des patients était le principal critère de jugement étudié. Le seuil de significativité retenu était la valeur de $\mathrm{p}<0,05$. 
Résultats: Au total, 126 patients étaient inclus, âgés en moyenne de $59+/-13$ ans avec une prédominance masculine (sex ratio $=1,45$ ). Le diabète, l'HTA et le tabagisme étaient les principales comorbidités notées pour $35,2 \%, 32 \%$ et $16,8 \%$, respectivement. Pour $80 \%(n=48)$ des patients à l'admission le taux de D-dimères étaient supérieurs à $500 \mu \mathrm{g} / \mathrm{l}$. La TDM thoracique à l'admission objectivait une atteinte sévère à critique pour $46,4 \% \quad(n=58)$. L'HBPM était prescrite pour $55,2 \%(n=69)$ et pour $44,8 \%(n=56)$, en prophylaxie et en traitement curatif, respectivement. Pour $17,6 \%(n=22)$ le devenir des patients était marqué soit par l'aggravation clinique nécessitant le transfert en réanimation $(16,7 \%, \mathrm{n}=21)$, soit par la survenue du décès $(0,8 \% ; \mathrm{n}=1)$. L'HBPM en prophylaxie avait un effet protecteur $(\mathrm{OR}=0,1[0,02$ à 0,3$]$, $\mathrm{p}$ value $<0,0001$ ), tandis que en traitement curatif, elle était associée à un risque d'aggravation (OR de 11 [3,3; 49], p <0,0001).

Conclusion: Il ressort de notre étude que l'HBPM en prophylaxie (mono dose de $4000 \mathrm{UI}$ ) avait un effet protecteur sur le risque de mortalité par COVID-19. Tandis qu'à dose curative (double dose de 6000 UI) elle était associée à un devenir péjoratif des patients.

Conflits d'intérêt: Rien à déclarer

\section{P204}

\section{Features of Asthma in Postmenopausal Women}

Rania Kaddoussi, Saoussen Cheikhm'hamed, Wafa Elfkih, Asma Migaou, Ahmed Ben Saad, Sameh Joober, Nessrine Fahem, Naceur Rouetbi

Service de penumologie CHU Fattouma Bourguiba Monastir

Introduction: Allergic asthma is the most common asthma phenotype. It is very sensitive to hormonal changes, especially in postmenopausal women.

Aim: To determine the characteristics of asthma in post-menopausal females.

Methods: Retrospective study carried out in the pneumology department of CHU Fattouma Bourguiba Monastir including 1,500 patients with allergic asthma confirmed by a skin test or specific Ige.

Among 903 women, sixty-five were postmenopausal at the time of data entry.

Results: The average age was 49.8 years with age ranges going from 40 to 79 years. All the women were non-smokers. Asthma was pre-existing in 47 women (72\%) and late onset in 18 women.

Personal and family atopy was noted in $87.7 \%$ and $60 \%$ of women, respectively. Rhinitis was associated with asthma in 29 patients $(44.6 \%)$. The sensitisation profile was dominated by pollen sensitisation in $40 \%$ of cases followed by dust mites in $34 \%$ of women. Monosensitisation was found in 21 women $(32.3 \%)$. Eight women required at least one hospitalization in a pulmonology department for exacerbation management and two in an intensive care unit. Pulmonary function tests found a fixed obstruc- tive ventilatory defect in 4 patients. Asthma was poorly controlled in 24 patients $(36.9 \%)$. Severe asthma requiring level 4 or 5 treatment was noted in 11 women (16.9\%) and level 3 treatment was required for $56.9 \%$ of patients.

Conclusion: Asthma during menopause is often poorly controlled, with severe exacerbations, hence the importance of a close monitoring.

\section{P205 \\ Une Année Après le Début de la Pandémie: Évaluation des Connaissances des Étudiants en Médecine Concernant la Covid 19}

\author{
Rania Kaddoussi, Issraa El Wadhane, Nessrine Fahem, \\ Cheyma Ben Rhouma, Asma Migaou, Ahmed Ben Saad, \\ Sameh Joober, Saoussen Cheikhm'hamed, Naceur Rouetbi
}

Service de penumologie CHU Fattouma Bourguiba Monastir

Introduction: Une année après la découverte du premier cas de covid 19, nos connaissances sur ce virus ne cessent de changer.

Les étudiants en médecine peuvent être impliqués dans la prise en charge des patients atteints de SARS COV2 d'où l'idée d'évaluer leurs connaissances sur cette pathologie.

Population et méthode: Une étude transversale était menée en ligne entre décembre 2020 et février 2021. Un questionnaire incluant 20 questions était élaboré et distribué au hasard aux étudiants en médecine.

Résultats: Cent huit étudiants ont répondu au questionnaire dont $51,9 \%$ étaient des femmes. Soixante-deux pour cent des participants appartiennent au premier cycle d'étude médicale.

Presque la totalité n'a pas fait de stage dans des unités covid 19. Le mode de transmission de ce virus était connu par 79 étudiants soit $73,1 \%$. Les participants avaient une connaissance moyenne du degré de contagiosité du SARS COV 19 (48,1\% ont connu le R0, $53,7 \%$ ont connu le niveau de protection des masques contre le covid $19,88 \%$ ont connu la durée moyenne de contagiosité et plus que la moitié $(53,7 \%)$ pensent qu'un auto-confinement est obligatoire après avoir été en contact avec un sujet malade indépendamment du degré de protection).

L'évaluation du respect des gestes barrières a objectivé que $88,9 \%$ des étudiants portent les masques et $61 \%$ utilisent une solution hydro-alcoolique.

Quarante-huit étudiants pensent qu'être jeune les épargne des formes graves de la covid-19.

Conclusion: Avec la persistance de cette épidémie et l'augmentation rapide du nombre des cas dans le monde, la connaissance des jeunes étudiants sur le covid 19 doit être améliorée vue leur exposition et leur rôle important dans la sensibilisation de leur entourage.

Conflits d'intérêt: Rien à déclarer
SSP/SSTS - EFP

Joint Annual Conference 2021 


\section{P206 \\ Attitudes et Comportements Tabagiques des Stagiaires en Médecine}

R. Jebali', S. Ben Saad ', A. Ben Mansour ', N. Guediri', S. Essebaa', I. Magroun ${ }^{2}$, H. Daghfous ${ }^{1}$, F. Tritar ${ }^{1}$

${ }^{1}$ Service de pneumologie C_Hôpital Abderrahmen

Mami_Ariana, ${ }^{2}$ Service de médecine de travail_Hôpital

Abderrahmen Mami_Ariana

Introduction: Le tabagisme constitue un problème majeur de santé publique à l'échelle mondiale. Il est responsable d'une morbimortalité importante. Malgré l'existence d'un programme de lutte antitabac, l'âge correspondant à la première cigarette est très jeune avoisinant 13 ans en Tunisie.

l'objectif: Evaluer le comportement tabagique, les connaissances et les attitudes des stagiaires en médecine vis-à-vis du tabac.

Méthode: Il s'agit d'une étude transversale, menée en mars 2020 incluant les stagiaires en médecine de l'hôpital Abderrahmane Mami. Un questionnaire leur a été accordé.

Résultats: Le taux de participation était de 68,53\% (61/89). Le sex ratio était de 0,27 . L'âge moyen était 22 ans. La prévalence globale du tabagisme était de $21,3 \%$. Elle était significativement plus élevée chez les femmes que chez les hommes (77\% vs 23\%) avec une moyenne de 5 cigarettes par jour. L'âge moyen de début du tabagisme était de 18 ans (17-22). Environ les $2 / 3$ des fumeurs déclaraient fumer à l'hôpital. Leurs raisons de fumer étaient le plaisir (77\%), l'automatique (39\%), le stress (39\%) et la meilleure concentration (15\%). La moitié des fumeurs avaient la volonté d'arrêter de fumer, $83,33 \%$ d'eux avaient tenté un sevrage. La majorité des étudiants $(85,2 \%)$ savaient que la publicité pour le tabac était interdite, $77 \%$ savaient que la vente aux enfants était interdite et 59\% savaient que fumer est interdit à l'hôpital. Tous les fumeurs savaient que le tabagisme était nocif.

Conclusion: La prévalence du tabagisme demeure élevée chez les stagiaires en médecine de sexe féminin. Des campagnes de sensibilisation et une consultation de sevrage tabagique à la faculté de médecine sont recommandées.

Conflits d'intérêt: Rien à déclarer

\section{P207 \\ Comportements et Attitudes Tabagiques Chez le Personnel de Santé}

R. Jebali', S. Ben Saad ', A. Ben Mansour ${ }^{1}$, I. Magroun' ${ }^{2}$, H. Daghfous ${ }^{1}$, F. Tritar ${ }^{1}$

${ }^{1}$ Service de pneumologie C_Hôpital Abderrahmen

Mami_Ariana, ${ }^{2}$ Service de médecine de travail_Hôpital

Abderrahmen Mami_Ariana

Introduction: Le tabagisme constitue un problème majeur de santé publique à l'échelle mondiale dont les coûts sociaux sont très élevés. Les professionnels de santé ont un rôle important à jouer dans la lutte anti-tabac. Ils sont en effet les premiers à sensibiliser, informer et aider au sevrage.

Objectif: Il est donc pertinent de déterminer la prévalence tabagique chez cette population particulière et ce afin d'étudier leur comportements et attitudes vis-à-vis du tabagisme.

Méthode: Etude descriptive transversale, menée en mars 2020 incluant les personnels de santé de l'hôpital Abderrahmane Mami. Un auto-questionnaire leur a été accordé.

Résultats: Il a été inclus 245 professionnels. Le taux de participation était de $85 \%$, Le sexe ratio était de 0,4 . L'âge moyen était 32 ans. Le personnel paramédical représentait 53\% $(\mathrm{n}=112)$, les étudiants en médecine 29\% $(n=61)$ et les médecins $18 \%(n=38)$. La prévalence du tabagisme était de $31,4 \%, \%$ chez les médecins, $22,6 \%$ chez les professionnels paramédicaux et $46 \%$ chez les étudiants en médecine. Une prédominance féminine a été notée chez les médecins fumeurs $(\mathrm{n}=14,70 \%)$. La moyenne de consommation tabagique était de 8 cigarettes par jour. L'âge moyen de début était de 18 ans (12-23 ans). Parmi les fumeurs, 75,5\% fumaient des cigarettes industrielles, $5 \%$ consommaient des cigarettes électroniques et $19,5 \%$ la Chicha. Environ les $2 / 3$ des fumeurs déclaraient fumer à l'hôpital. Leurs raisons de fumer étaient le plaisir (69\%), l'automatique (44\%), le stress $(50,7 \%)$ et la meilleure concentration $(13,8 \%)$. La moitié des fumeurs avaient la volonté d'arrêter de fumer dont 53,8\% avaient tenté un sevrage. Près de $74 \%$ du personnel soignant savaient que la publicité pour le tabac était interdite, $79 \%$ savaient que la vente aux enfants était interdite et $60 \%$ savaient que fumer est interdit à l'hôpital. Tous les fumeurs savaient que le tabagisme était nocif.

Conclusion: Le taux de tabagisme est élevé chez le personnel soignant. Des compagnes de sensibilisation de même que des consultations de sevrage tabagique doivent cibler cette population afin de tenter de réduite les impacts à moyen et à long terme de ce fléau.

Conflits d'intérêt: Rien à déclarer

\section{P208 \\ L'extension Pulmonaire Initiale de la Pneumonie COVID-19: Facteur Prédictif D'aggravation Secondaire?}

R. Jebali ${ }^{1}$, S. Louhaichi' , I. Khalfallah', Safa Marzouki ${ }^{1}$, Line Kaabi ${ }^{1}$, Sabrine Elfidha ${ }^{1}$, Mariem Affes ${ }^{2}$, Jamel Ammar ${ }^{1}$, Basma Hamdi', A. Hamzaoui ${ }^{1}$

${ }^{1}$ Service de pneumologie B, Hôpital Abderrahmen Mami Ariana

${ }^{2}$ Service de radiologie Hôpital Abderrahmen Mami Ariana

Introduction: La pneumonie au SARS-CoV2 se caractérise par un tableau clinique variable allant des formes légères sans gravité jusqu'aux formes critiques mettant en jeu le pronostic vital. Le scanner thoracique initial permet d'estimer l'étendue de l'atteinte parenchymateuse pulmonaire. Mais cette évaluation initiale permet-elle de prédire l'évolution secondaire de l'infection?

Objectif: Evaluer la valeur pronostique de l'estimation scannographique des lésions pulmonaires faite à l'admission chez des patients hospitalisés pour pneumonie COVID-19. 
Méthode: Il s'agit d'une étude rétrospective incluant 270 patients hospitalisés entre octobre 2020 et mars 2021dans le département de pneumologie B de l'hôpital Abderrahman Mami pour pneumonie COVID-19. L'atteinte pulmonaire a été classée par les radiologues en: minime $(<10 \%)$, modérée $(10-25 \%)$, étendue $(25-50 \%)$, sévère $(50-75 \%)$ ou critique $(>75 \%)$. Le critère de jugement principal était l'aggravation clinique à j15 d'hospitalisation, définie par un transfert secondaire en réanimation ou un décès.

Résultats: La médiane d'âge était de 66 ans [18-92ans] avec un sexe ratio égal à 1,32 . Le tabagisme était présent dans $47 \%$ des cas. La durée moyenne d'évolution des symptômes était de 9jours [2-14]. L'atteinte scannographique était qualifiée de minime, modérée, étendue, sévère ou critique chez 8 (3\%), 33 (12\%), 80 (30\%), $105(39 \%)$, et $44(16 \%)$ patients, respectivement. L'évolution était favorable dans $80 \%$ des cas. Quarante-deux patients ont été transférés en réanimation et douze sont décédés. La durée moyenne d'hospitalisation n'était pas corrélée à l'extension lésionnelle pulmonaire. Néanmoins, une atteinte pulmonaire $>50 \%$ était associée à un pronostic plus sombre, avec plus de transfert en réanimation $(20.8 \%$ versus $9 \%, \mathrm{p}=0.008)$ et une mortalité plus élevée (6.7\%versus1.6\%, $\mathrm{p}=0.04$ ).

Conclusion: Le scanner thoracique aurait un intérêt pronostique permettant le triage des patients à risque de formes sévères. Une atteinte lésionnelle $>50 \%$ était associée à une aggravation secondaire.

Conflits d'intérêt: Rien à déclarer

\section{P209 \\ Traitement Chirurgical des Thymomes par Vidéothoracoscopie Exclusive: Expérience Tunisienne}

\author{
R. Essid' , I. Bouassida ${ }^{1}$, M. Abdennadher ${ }^{1}$, H. Zribi ${ }^{1}$, Y. Houcine ${ }^{2}$, \\ S. Ouerghui ${ }^{3}$, A. Merghli $^{1}$ \\ ${ }^{1}$ Service de chirurgie thoracique et cardio vasculaire hôpital \\ Abderrahmen mami Ariana, ${ }^{2}$ Service d'anatomopathologie \\ hôpital Abderrahmen mami Ariana, ${ }^{3}$ Service d'anesthésie \\ réanimation hôpital Abderrahmen mami Ariana
}

Introduction: Les tumeurs thymiques occupent la première place dans la pathologie tumorale du médiastin antéro-supérieur. Les thymomes sont des tumeurs à développement lent. Ils sont alors peu symptomatiques parfois découverts fortuitement. Le traitement de référence est la résection radicale chirurgicale. $\mathrm{La}$ sternotomie médiane reste la voie d'abord de référence, cependant les voies mini-invasives sont de plus en plus utilisées surtout avec les améliorations du matériel chirurgical endoscopique.

Matériel et méthodes: Il s'agit d'une étude rétrospective descriptive de 2015 à 2020 portant sur 10 dossiers de patients ayant eu une résection chirurgicale d'un thymome par vidéothoracoscopie exclusive (VTS) au service de chirurgie thoracique et cardio vasculaire de Abderrahman mami.
Résultats: Il s'agissait de 4 hommes et 6 femmes, la moyenne d'âge était de 52 ans [16-81]. 8 patients avaient une myasthénie sous traitement médical. Un patient avait une douleur thoracique et un patient était asymptomatique. La radiographie du thorax était normale chez 2 patients, et elle montrait un élargissement médiastinal chez 8 autres. Un scanner thoracique a été fait chez tous les patients montrant une opacité médiastinale antérieure bien encapsulée dont la taille ne dépassait pas de $6 \mathrm{~cm}$, sans envahissement locorégional. 6 patients ont été opéré par VTS droite et 4 par VTS gauche, selon la latéralisation de la masse. Le geste consistait en une thymectomie élargie à la graisse médiastinale. Une conversion en sternotomie a été faite pour un seul patient car la masse envahissait le péricarde. La durée moyenne du drainage thoracique était de 2 jours et la durée moyenne d'hospitalisation était de 3.7 jours. Les suites opératoires étaient simples pour tous les patients. Aucun patient n'a eu une poussée myasthénique en post opératoire. Une réduction des doses d'inhibiteur de l'acétylcholinestérase a été observée chez deux patients myasthéniques. Toutes les limites de résection étaient saines. 9 patients avaient un stade II de masaoka à l'examen anatomopathologique et un patient avait un stade I. Aucun patient n'a eu une récidive locale ou à distance avec un recul maximal de 5 ans.

Conclusion: La vidéothoracospie trouve de plus en plus sa place dans la prise en charge des thymomes de petites tailles, bien encapsulées, sans envahissement locorégional, associées ou non à une myasthénie. Elle a un avantage esthétique avec diminution des douleurs post opératoires, et de la durée d'hospitalisation. Les résultats oncologiques et thérapeutiques sont satisfaisants.

Conflits d'intérêt: Rien à déclarer

\section{P210 \\ Profil Épidémiologique, Clinique, Radiologique et Évolutif de la Silicose Pseudotumorale}

\section{Saidi, S. AitBatahar, L. Amro}

Service de Pneumologie, Hôpital ARRAZI, CHU Mohammed VI, UCAM, FMPM, Marrakech

Introduction: La forme pseudo-tumorale de la silicose est une entité rare posant un problème diagnostic différentiel avec le carcinome bronchique.

Méthode: Étude rétrospective portant sur 14 cas de silicose pseudotumorale colligés au service de pneumologie du CHU Mohammed VI de Marrakech entre Janvier 2013 et Janvier 2021.

Résultats: L'atteinte était exclusivement masculine avec une moyenne d'âge de 55 ans. Nous retrouvons comme profession dominante: puisatier dans $42,8 \%$ des cas, maçon dans $35,7 \%$ des cas. Le tabagisme actif était retrouvé dans 12 cas. La symptomatologie était dominée par la toux et la dyspnée d'effort chez tous nos patients et l'hémoptysie dans $28,5 \%$ des cas. La radiographie thoracique avait montré des opacités macronodulaires confluentes réalisant des images pseudo-tumorales bilatérales dans 78,5\% des cas, associées à des opacités micronodulaires diffuses dans 14,2\% des cas et à un pneumothorax dans un cas. La TDM thoracique avait objectivé de multiples masses macronodulaires associées à 
des adénopathies calcifiées sous-carinaires et hilaires dans $85,7 \%$ des cas et un épaississement pleural avec des plaques sous pleurales dans $14,2 \%$ des cas. La bronchoscopie avait retrouvé un état inflammatoire diffus chez 9 cas associé à des taches anthracosiques dans 5 cas sans signes de malignité. La recherche de BK dans les expectorations et dans le liquide d'aspiration bronchique était négative dans tous les cas. L'exploration fonctionnelle respiratoire avait objectivé un TVO non réversible dans $85,7 \%$ des cas. Le diagnostic de silicose pseudo-tumorale était retenu devant le contexte clinique évocateur et l'aspect pseudo-tumoral des lésions radiologiques. L'évolution était bonne dans 9 cas, 2 patients étaient décédés et trois patients étaient perdus de vue.

Conclusion: La silicose est l'une des pneumoconioses les plus répandues entrainant un retentissement fonctionnel important, sa forme pseudotumorale est source de difficulté diagnostic chez le clinicien.

Conflits d'intérêt: Rien à déclarer

\section{P211 \\ Apport de L'endoscopie Rigide Dans la Prise en Charge des Tumeurs Malignes Proximales Pulmonaires}

Animi. Salma, Zouiten. Salim, Rjimati. Manelle, Essabahi. Btissame, Serraj. Mounia, Amara. Bouchra, Benjelloun. Mohammed Chakib

Service de Pneumologie, CHU Hassan II FES

Introduction: L'endoscopie interventionnelle assure un traitement palliatif rapide des obstructions bronchiques tumorales proximales en utilisant la résection mécanique au tube rigide ou la pose de prothèses en silicone en cas de compressions extrinsèques.

Méthode: La présente étude est rétrospective étalée sur une période de 3 ans, de janvier 2019 - avril 2021. Elle a été menée au service de pneumologie du CHU Hassan II de Fès.

Le but de notre étude est de mettre en évidence les indications et intérêts thérapeutiques palliatifs de l'endoscopie bronchique rigide pour les tumeurs malignes pulmonaires proximales.

Résultats: La bronchoscopie rigide est indiquée en cas d'obstruction tumorale de l'arbre bronchique ou de compression extrinsèque. Une bronchoscopie souple préalable est primordiale pour explorer les caractéristiques de l'obstruction. La désobstruction bronchique ou la pose de prothèse par bronchoscopie rigide nécessite d'avoir des voies aériennes fonctionnelles en aval de l'obstruction.

La symptomatologie était dominée par les hémoptysies et la dyspnée, radiologiquementils'agissaitle plus souvent d'atélectasies. L'obstruction dépassait $50 \%$ de la lumière bronchique chez la majorité des patients. En cas de lésions bourgeonnantes, une désobstruction bronchique par résection mécanique avec le bec du bronchoscope rigide a été réalisée. En l'absence de compression extrinsèque, l'intérêt d'une prothèse après désobstruction bronchique en prévention d'une rechute locale est discuté. La compression extrinsèque est l'indication privilégiée de la pose de prothèse de DUMON. Nous avons utilisé des prothèses en silicone dites: droites pour la trachée ou les bronches principales ainsi que des prothèses en $\mathrm{Y}$ pour la carène, sur mesure ou à diamètre variable.

Les complications les plus fréquentes étaient les infections respiratoires basses et les resténoses tumorales. L'amélioration de la dyspnée était constatée chez $80 \%$ de nos patients.

Conclusion: La bronchoscopie rigide permet d'améliorer la dyspnée des patients atteints d'une obstruction tumorale trachéobronchique proximale.

Conflits d'intérêt: Rien à déclarer

\section{P212 \\ Profil des Comorbidités des Patients Covid-19 Hospitalisés Dans I'unité de Soins Intensifs de CHU Mohamed VI de Marrakech.}

\section{S. Kechnaoui, S. Aitbatahar, L. Amro}

Service de pneumologie. Labo. PCIM, UCA, Marrakech, Maroc

Introduction: La présence de conditions médicales préexistantes chez les cas de la COVID-19 a été identifiée comme un facteur de risque de complications pouvant mener au décès. But de travail: Le but de notre travail était d'étudier le profil des comorbidités des patients Covid-19 hospitalisés dans l'unité de soins intensifs de CHU Mohamed VI de Marrakech.

Matériels et méthode: C'est une étude rétrospective, descriptive monocentrique incluant les cas d'infection Covid-19 hospitalisés dans l'unité de soins intensifs du CHU Mohamed VI de Marrakech entre octobre et décembre 2020.

Résultats: on a colligé 122 cas; Il s'agit de 73 hommes (59.8\%) et 49 femmes (40.2\%). La moyenne d'âge des patients était de 56.7 ans \pm 14.59 avec des extrêmes de 24 et 87 ans. Les patients âgés de plus de 60 ans représentaient $51 \%$ des cas. Trente patients n'avaient pas de comorbidité $(24.6 \%)$ et 92 avaient au moins une comorbidité (75.4\%) dont 51 avaient une seule comorbidité (41.8\%), 26 avaient deux comorbidités (21.3\%) et 15 avaient trois comorbidités ou plus (12.3\%). Les comorbidités trouvées étaient essentiellement le diabète chez 48 cas (39.3\%), l'HTA chez 40 cas (32.8), une maladie cardiovasculaire chez 11 cas (9\%), une néoplasie chez 9 cas (7.3\%) et une dysthyroidie chez 9 cas $(7.3 \%)$.

Conclusion: les patients hospitalisés en unité de soins intensifs Covid-19 sont essentiellement des patients âgés ayant une ou plusieurs comorbidités.

Conflits d'intérêt: Rien à déclarer 


\section{P213 \\ Étude de l'impact du SAOS sur L'équilibre}

Senda Turki, Fatma Ajlani, Ferdaous Yangui*, Hamdi Chouchane, Chiraz Halwani, Amani Hachicha, Med Ridha Charfi*

Service d'ORL et de Chirurgie Cervico-faciale de l'Hôpital des FSI de la Marsa, *Service de Pneumologie de l'Hôpital des FSI de La Marsa

Introduction: Le Syndrome d'Apnées Obstructives du Sommeil (SAOS), associé à un risque élevé de complications cardiaques et vasculo-cérébrales, peut léser le labyrinthe dont la vascularisation est de type terminal.

Objectif: rechercher l'impact du SAOS sur le système vestibulaire, par l'étude du Reflexe Vestibulo Oculaire (RVO) dans des conditions physiologiques en utilisant le video Head Impulse Test (vHIT) et évaluer l'effet du traitement par Pression Positive Continue (PPC) par un deuxième test de vHIT.

Méthode: Cette étude prospective (Novembre 2018 - Décembre 2020), a inclus des patients porteurs d'un SAOS modéré à sévère. Un premier vHIT a été initialement réalisé à tous les patients, et un deuxième vHIT après PPC à 11 patients. Un groupe témoin a juste bénéficié d'un vHIT à titre comparatif avec les patients.

Résultats: 14 patients d'âge moyen de 54,7 ans (38-73) et de Sex Ratio à 2 et 21 témoins d'âge moyen de 53 ans (34-80) et de Sex Ratio à 1,6. Le score d'Epworth moyen était de 13 (5-21). L'IAH moyen était de 48 (19-86). Des troubles importants de l'équilibre ont été signalés par $40 \%$ des patients. Au vHIT, le gain moyen des patients était dans les normes, mais significativement meilleur chez les patients sans troubles cognitifs. Les gains des patients étaient significativement diminués par rapport aux témoins. Le deuxième vHIT réalisé à 11 patients, après utilisation moyenne de la PPC de 209 jours (32-646), un IAH résiduel moyen de 0,6 (0-2,4) et une disparition clinique des troubles de l'équilibre n'a pas montré d'amélioration significative des gains.

Conclusion: Le SAOS majore le risque de troubles de l'équilibre et altère les gains du RVO. Le traitement par PPC, améliorant les symptômes mais sans effet sur la valeur des gains, favoriserait vraisemblablement la compensation centrale.

Conflits d'intérêt: Rien à déclarer

\section{P214}

\section{Connaissances, Attitudes et Pratiques des} Professionnels de la Santé sur la COVID-19 au Togo

\section{Tété Amento Stéphane Adambounou ${ }^{1,2}$, Doevi Godwin Biaou', Pwemdeou Efalou ${ }^{3,4}$, Lidaw Bawe ${ }^{2,5}$, Komi Séraphin Adjoh ${ }^{1,2}$}

${ }^{1}$ Service de pneumologie, CHU Sylvanus Olympio, Lomé-Togo, ${ }^{2}$ Faculté des sciences de la santé, Université de Lomé, LoméTogo, ${ }^{3}$ Service de pneumologie, CHU Kara, Kara-Togo, ${ }^{4}$ Faculté des sciences de la santé, Université de Kara, Kara-Togo; ${ }^{5}$ Service des maladies infectieuses, CHU Sylvanus Olympio, Lomé-Togo

Introduction: L'épidémie virale apparue en Chine, nommée COVID-19, est rapidement devenue une pandémie. En mai 2020, on recensait 4 millions de cas avec 275.000 décès. Les principaux acteurs de la lutte contre cette maladie, hautement contagieuse mais méconnue du monde scientifique, étaient les professionnels de la santé (PS). Il était opportun d'évaluer le niveau de connaissance des PS au Togo sur cette épidémie et d'apprécier leurs attitudes et pratiques.

Méthode: il s'est agi d'une enquête transversale à visée descriptive et analytique menée du 29 Mars au 05 Avril 2020 à l'aide d'un questionnaire électronique. Il a été soumis via les réseaux sociaux sur la plate-forme des médecins et celle des paramédicaux. La participation était libre. L'anonymat a été assuré par la codification des fiches d'enquête. Quatorze questions permettaient d'évaluer le niveau de connaissance.

Résultats: 130 personnes, âgées en moyenne de 31,9 $\pm 6,5$ ans, ont participé à l'enquête. Il s'agissait de médecins $(67,7 \%)$ et d'infirmiers $(23,8 \%)$. L'expérience professionnelle était entre 5 10 ans dans $28,5 \%$ des cas. Les gouttelettes salivaires infectantes $(96,2 \%)$ et le contact avec les surfaces contaminées $(85,4 \%)$ étaient les principales voies de contamination. La définition de cas présomptif était connue de 46,2\%. Les symptômes évocateurs étaient une toux $(93,8 \%) \pm$ une dyspnée $(88,5 \%)$ aigues avec fièvre $(97,7 \%)$. Le traitement était symptomatique selon $92,3 \%$ des répondants. Selon 38,5\% répondants, les PS devraient disposer d'un équipement de protection individuelle (EPI) complet. 56,2\% des répondants étaient disposés à travailler dans une unité de prise en charge des cas de Covid-19.

Conclusion: Le niveau de connaissance général était moyen. Une remise à niveau des PS sur la Covid-19 et la mise à disposition d'EPI s'avèrent nécessaires afin de les rassurer et leur permettre d'exercer sereinement leurs professions.

Conflits d'intérêt: Rien à déclarer

\section{P215 \\ Evaluation de l'impact du Tabagisme au Cours de la Pneumopathie à SARS-COV2}

\author{
Hedhli Abir, Jelassi Warda, M. Mjid, A. Allouche, Ouahchi Yacine,
} S. Merai, S. Toujani, B. Dhahri

Service de pneumologie LR18SP02 Hôpital La Rabta Tunis, Tunisie

Introduction: Le tabac contribue à l'altération des défenses immunitaires des voies respiratoires et rend les fumeurs plus sensibles aux infections pulmonaires et donc théoriquement au SARSCoV2. Les données intéressant l'étude des liens entre le tabagisme et la sévérité de la pneumopathie à SARS-CoV2 restent divergentes. L'objectif de notre étude était d'évaluer l'impact du tabagisme sur la sévérité et l'évolution de la pneumopathie à SARS COV2.

Méthode: Nous avons mené une étude rétrospective portant sur 150 malades hospitalisés dans notre service pour pneumopathie à SARS COV2 sévère. Les données clinico-biologiques et radiologiques ont été recueillies à partir des dossiers médicaux. L'évolution péjorative était définie par la survenue d'une complication, le passage en milieu de réanimation ou le décès. Une
$744 \quad$ Respiration $2021 ; 100: 644-750$
DOI: $10.1159 / 000517200$
SSP/SSTS - EFP

Joint Annual Conference 2021 
atteinte radiologique sévère était définie par une étendue des lésions $>50 \%$ du parenchyme pulmonaire.

Résultats: La population étudiée comportait 81 (54\%) femmes et $69(46 \%)$ hommes, avec un âge moyen de $54.84 \pm 14$ ans. Un tabagisme actif a été trouvé chez 38 patients (25\%) avec une nette prédominance masculine et une consommation moyenne de paquets/année. Les patients tabagiques avaient une saturation périphérique initiale en oxygène statistiquement plus abaissée (87 versus $91.6 \% ; \mathrm{p}<10-3)$ et des besoins en oxygène plus importants ( 8 versus 6,3 litres/minutes; $\mathrm{P}<10-3$ ). Le tabagisme était associée à la présence d'une atteinte radiologique sévère $(\mathrm{p}=0.003)$. Sur le plan biologique, l'intensité du tabagisme était positivement corrélée à la C-Reactive Protein $(\mathrm{p}=0.009)$. Aucune corrélation n'a été retrouvée avec le taux des lymphocytes $(\mathrm{P}>0,05)$.

Conclusion: Notre étude a montré que tabagisme était associé à une forme plus sévère de pneumopathie type covid19. Néanmoins, une étude multicentrique à grande échelle s'avère indispensable afin de confirmer ces constatations.

Conflits d'intérêt: Rien à déclarer

\section{P216 \\ Impact of Continuous Positive Airway Pressure Therapy on Echocardiographic Findings in Obstructive Sleep Apnea patients}

Touil.I', H. Ibn Hadj Amor ${ }^{2}$, Y. Brahem ${ }^{1}$, N. Keskes Boudawara', S. Bouchareb ${ }^{1}$, R. Chebbi ${ }^{2}$, S. Kraiem ${ }^{2}$, H. Baili', S. Ksissi ${ }^{1}$, J. Ayeb ${ }^{1}$, I. Bouchnak ${ }^{2}$, L. Boussoffara' ${ }^{1}$ J. Knani ${ }^{1}$

'Department of pneumology, Taher Sfar Hospital, 5100 Mahdia, TUNISIA, ${ }^{2}$ Department of Cardiology, Taher Sfar Hospital, 5100 Mahdia, TUNISIA

Introduction: Obstructive sleep apnea (OSA) may influence the cardiac function by several mechanisms. However, adequate treatment of the respiratory disturbance through CPAP may improve and even reverse these structural and functional alterations of both left and right ventricles.

Aim of the study: We aimed to investigate echocardiographic abnormalities associated with severe OSA and determine the effect of device therapy.
Materials and Methods: We conducted a cross-sectional observational and analytic study, including patients who were performed a respiratory polygraphy in the sleep laboratory of the Pneumology Department of Taher Sfar Hospital. A standard 2D echocardiography and conventional Doppler were performed. According to device therapy adherence severe OSA patients were divided into three groups: G1: Severe untreated OSA patients. / G2: Severe treated and adherent OSA patients: those who used the device for at least 4 hours per night for at least $70 \%$ of the days. I G3: Severe treated but non-adherent OSA patients. Echocardiographic parameters were analyzed in healthy obese subjects and in all subgroups severe OSA patients.

Results: A total of 139 patients was diagnosed with confirmed OSA (AHI $>=5$ events $/ \mathrm{h}$ ). According to AHI, 67 patients were diagnosed with severe OSA with $A H I \geq 30$ events/h and symptomatic patients with AHI $<5$ events/h served as a control group $(n=45)$. There is no difference between control group and severe OSA group concerning demographic, clinical, biological and cardiopulmonary function. Echocardiographic analysis showed an enlargement right ventricular internal diameter (RVID) and a higher a right ventricular wall thickness (RVWT) in severe OSA patients compared to controls, with $\mathrm{p}<0.001$ and $\mathrm{p}=0.017$ respectively. Similarly, sPAP values were significantly higher in severe OSA patients compared to controls $(30,5 \pm 10,8$ vs. $20.9 \pm 9.8 ; \mathrm{p}<0.001)$. The RV function as displayed via TAPSE was significantly lower in the severe OSA patients compared to controls $(\mathrm{p}<0.001)$. Concerning the LV, the LVEF was lower in severe OSA patients than in controls $(p=0.025)$ but a higher prevalence of left ventricular diastolic dysfunction (LVDD) among severe OSA patients (68.6\% vs $15.9 \%$; $\mathrm{p}<0.001)$. In our OSA population, CPAP was used by 45 severe OSA patients (67.2\%): 23 OSA patients were adherent users (51\%) and 22 non-adherent users (49\%). Twenty two patients were untreated. No significant difference in the echocardiographic features (RVID, RVWT, sPAP, TAPSE, E/A, IVRT, DT) was observed either between all severe OSA subgroups.

Conclusion: OSA was associated with RV remodeling and dysfunction. A persistent RV dilatation with a dysfunction trend under adequate CPAP treatment, which raises a red flag inciting an adequate therapy at subclinical stages.

Disclosure: Nothing to disclose 


\section{Respiration}

Abdala S. P15, P16, P24, P39, P68

Abdraeva A. P42, P45, P48

Adè S. P51

Adler D. P08, P11, P43, P52

Aeschbacher S. P26, P27

Agodokpessi G. P51

Aitbatahar S. P15

Akujinwa I. P54

Akylbekov A. P47

Alovokpinhou F. P51

Amacker M. P30

Amro L. P15

Annaheim S. P50

Appenzeller P. P32, P45, P48

Aubert J.-D. P32

Aydaralieva S. P42, P45

Azzola A. P32

Bader P. P45

Barata F. P13

Battilana B. P63

Baty F. P13, P41, P49, P50, P64

Ben Mansour A. P67

Ben Radhia B. P67

Ben Saad A. P36

Bengueddache S. P23

Berger M. P40

Berlier C. P32, P33, P35, P38

Bettex D. P63

Betticher D. P59

Beyer S. P04, P28

Bezel P.R. P57

Biaggi C. P59

Bitos K. P42, P47

Blanchon S. P14

Blank F. P17, P30

Bloch K.E. P26, P27, P35, P37, P38, P42, P45, P47, P48

Boesch M. P13, P50, P64

Bonnier G. P50

Bouali R. P09

Bouassida I. P67

Braun F. P50

Braun J. P04

Bridevaux P.-O. P09, P25

Bringard A. P31

Brun P. P04

Brutsche M. P41, P49, P50

Brutsche M.H. P13, P64

Buenzli S. P42

Buergin A. P42, P45, P47
Numbers refer to Abstract numbers
Bullo M. P19

Bürki E. P41

Büsching G. P04

Calendo L.-R. P38

Cantero C. P11, P52

Carron T. P01

Carta A.F. P35, P38

Casaulta C. P19

Caviezel C. P55

Cazaentre T. P23

Cerini T. P06

Charlotte B. P37

Cheikh Mhammed S. P36

Chirindel A.-F. P61

Clarenbach C.F. P03

Cleres D. P13

Collaud E. P18, P22

Corbach N. P33

Crisinel P.A. P14

Curioni-Fontecedro A. P59

Daccord C. P48

Dalla Lana K. P01, P04, P06

Dridi A. P67

Dupuis-Lozeron E. P11

Eboulet E.I. P59

Egger B. P52

Eloued L. P36

Fahem N. P36

Faouzi M. P20

Fellrath J.-M. P32

Ferrario D. P50

Ferrié C. P17, P30

Fiogbé A. P51

Fleisch E. P13

Flueck D. P27

Fontolliet T. P31

Fouraki A. P14

Franzen D. P57, P60, P66

Frei A. P01, P04, P06

Frésard I. P09, P25

Frueh M. P59

Früh M. P64

Furian M. P26, P27, P35, P37, P42, P45, $\mathrm{P} 47, \mathrm{P} 48$

Furrer K. P56, P58, P59, P63

Furrer M. P59

Fytianos K. P17
Gasche P. P08, P52

Gasche-Soccal P. P31

Gaspert A. P66

Gautschi F. P33, P38, P66

Gazdhar A. P07, P17

Geiser T. P07, P32

Genecand L. P09, P25

Gex G. P09, P25

Giroud P. P01

Gobin N. P09

Goutaki M. P18, P22

Greutmann M. P38

Grimaldi S. P60

Grosgurin O. P11

Groth A. P33, P47

Guerin S. P14

Guerreiro I. P31, P43

Guetari L. P36

Guler M. P01

Haba-Rubio J. P40

Hage R. P66

Hasler E.D. P26, P27

Hayoz S. P59

Hebeisen M. P63

Heinzer R. P40, P51

Hermann M. P02

Hetzel J. P28, P46, P53

Hillinger S. P55, P56, P58

Hofer M. P28, P46, P53

Hojski A. P61

Horisberger T. P63

Houinato D. P51

Huber F. P26, P27

Hüsler L. P18, P22

Iliev E. P56

Inci I. P55, P56, P58, P63

Jaksic C. P52

Janssens J.-P. P08, P10, P11, P12, P43, P52

Jobeur S. P36

Joerger M. P64

Kaddoussi R. P36

Kharat A. P11

Kieninger E. P19

Knippschild S. P05

Kohlbrenner D. P03

Kohler M. P44

Kopp G. P31

Koppe H. P18
Kowalski B. P57

Krähenmann F. P33

Krueger T. P59

Kuehni C. P18, P22

Kuhn M. P03

Kunz R. P04, P06

Lador F. P11, P31, P32

Lam Y.T. P18, P22

Lardinois D. P59, P61

Lassoued T. P36

Latshang T.D. P26, P27

Latzin P. P19

Lauk O. P55

Lawi D. P25

Lazor R. P20, P48

Lemay M. P50

Lenoir A. P20

Lhonneux C. P08

Lichtblau M. P26, P27, P32, P33, P35, P38, P42, P45, P47, P48

Lolachi S. P11

Luu P. P05

Luyken M.C. P48

Mademilov M. P42, P45, P47, P48

Mahmoudi E. P36

Mallaev M. P61

Maltor C. P08

Mangold M. P60

Marazhapov N. P48

Marazhapov N.H. P42, P47

Marghli A. P67

Marques-Vidal P. P40

Marsland B.J. P29

Marti C. P11

Matter A. P63

Mayer L. P42, P45, P47

Mayer L.C. P37

Messe R. P31

Meszaros M. P27, P44

Migaou A. P36

Mirzalieva G. P47

Mona L. P37

Monsch G.-M. P55

Morselli H.-T. P63

Moser M. P49

Müller J. P35, P38

Müller L. P19

Muller M.-E. P21

Muralt L. P45, P47 
Muratbekova A. P42, P45, P48

Mutlu S. P17, P30

Nagaraj N.G. P63

Nicod L.P. P29

Nicolas G. P61

Nido M. P44

Nordmann Y. P03

Oleinik A. P47

Opitz I. P32, P55, P56, P58, P59, P63

Ott N. P46, P53

Ozan V.B. P07

Paciorkowski M. P41

Paquier C. P20

Pasquina P. P52

Pedersen E. P18, P22

Pekacka-Egli A.-M. P02

Pepin J.-L. P52

Pereitti V. P14

Perentes J. P59

Pernot J. P29

Peters S. P59

Peytremann-Bridevaux I. P01

Pless M. P59

Plojoux J. P11, P12

Pohle S. P32, P41

Polhemus A. P06

Poncet A. P10, P12, P52

Prella M. P52

Proença M. P50

Proust Y.-M. P50

Puhan M. P01, P04, P06

Radtke T. P04, P06

Rassouli F. P13
Reinhard L. P27

Reiser A. P42

Reiser A.E. P45

Renevey P. P50

Renzi G. P10, P12

Rezek S. P28, P46, P53

Riegler T. P04

Rochat I. P14

Rothschild S. P59

Rouatbi N. P36

Rozier Aubry B. P20

Rüber F. P60

Rüschoff J. P66

Sadowski A. P08

Saidani W. P67

Sauty A. P23

Savas S.T. P19

Saxer S. P26, P32, P33, P35, P37, P38

Scalise M. P30

Scheiwiller P.M. P26, P27, P47, P48

Schmid O. P63

Schmid R.A. P59

Schmiedeskamp M. P46, P53

Schmit A. P43

Schmuziger Y.A. P47

Schneider S.R. P26, P35, P37, P42, P45, P47

Schneiter D. P55, P56, P58

Schoch O.D. P49, P50

Schoendorf S. P02

Scholtze D. P57

Schöndorf S. P04

Schrenzel J. P10, P12

Schuepbach R. P63

Schüpbach R. P33

Schuurmans M. P66
Schwarz E. P43

Schwarz E.I. P27, P33, P35, P37, P38, P44

Schwerzmann M. P32

Sheraliev U. P42, P47, P48

Shih I. P13

Siclari F. P40

Sigrist T. P04

Soccal P.M. P11, P43

Sooronbaev T.M. P42, P45, P47, P48

Spielmanns M. P02, P03, P04, P05

Stahel R.A. P59

Steier J. P44

Steinack C. P60, P66

Steiner S. P17, P30

Steiner U.C. P57

Steurer-Stey C. P01

Stolz D. P61

Strassmann A. P01

Stricker H. P32

Stupp R. P59

Stüssi-Helbling M. P03

Taffe P. P48

Takahashi H. P08

Tamm M. P32, P61

Tan L. P37

Tanniger J. P20

Theurillat P. P50

Thierstein S. P59

Tinschert P. P13

Totah T. P51

Trompette A. P29

Truong M.K. P40

Tschanz S. P19

Tschirren A.-B. P17

Tulka S. P05
Ubags N. P29

Uldry C. P52

Ulrich S. P26, P27, P32, P33, P35, P37, P38, P42, P44, P45, P47, P48, P63

Unger I. P28, P46, P53

Valaperti A. P57

Van Zaen J. P50

Vaudaux A. P08

Veziris N. P12

Volk G. P46, P53

Von Garnier C. P29, P30

Vongthilath-Moeung R. P10, P12

Vremaroiu Coman A. P25

Vremaroiu P. P25

Wachinou A.P. P51

Weder W. P56, P59

Werner R. P58

Wiese M. P61

Wieser S. P57

Windisch W. P02, P05

Wotzkow C. P17, P30

Younossian A.B. P52

Zairi S. P67

Zribi H. P67 


\section{Respiration}

Aazri L. P141

Abdala S. P170

Abdelaziz H. P153, P154

Abdelghani A. P91, P92, P93, P94, P116, P117

Abdelkebir A. P193

Abdennadher M. P96, P103, P193, P209

Abderraouf A. P144

Abid A. P164, P165, P166

Abid N. P136, P137, P215

Adambounou A.S. P146

Adambounou T.A.S. P214

Ade S. P71

Adila F. P72

Adjoh K.S. P214

Afef B. P184, P185, P186, P187

Affes P. P208

Afif H. P69, P77, P78, P79, P80, P81, P84, P85, P101, P102, P106, P109, P110, P111, P112, P113, P114, P115

Afif M.H. P120, P121, P122

Agnès H. P108, P134

Agodokpessi G. P71

Ahmed A. P153, P154

Ahsino F. P164, P165, P166

Aichaouia C. P181, P182, P183

Aidou S. P106, P110, P111, P112, P113, P114, P115

Aissa S. P91, P92, P93, P94, P116, P117

Ait batahar S. P141, P157, P158, P190, P192, P197, P198, P199, P203, P210, P212

Ait Batahar S. P190, P192, P197, P198, P199, P203, P210, P212

Ait mouddene N. P69

Aitbatahar S. P139, P140, P142, P170

Ajim A. P69, P80

Ajlani F. P213

Alain K. P70

Allouch A. P82, P131

Allouche A. P188, P189, P215

Amar J.B. P151, P152, P179

Amara B. P211

Amara B. P74, P156

Ammar J. P104, P208
Numbers refer to Abstract numbers
Ammar Jamel J.R. P134

Ammar N. P105

Amraoui M. P163

Amro L. P139, P140, P141, P142, P157, P158, P168, P169, P170, P171, P190, P192, P197, P198, P199, P203, P210, P212

Andriamihaja R. P87

Andrianarisoa A. P87

Andrianasolo R.M. P135

Animi S. P211

Anniche H. P73

Aouina H. P82, P83, P130, P131, P151, P152, P179, P188, P189

Aoun-Bacha Z. P133

Arfaoui H. P69, P77, P78, P79, P80, P81, P84, P85, P101, P102, P109, P110, P111, P112, P113, P114, P115, P120, P121, P122

Asma K. P153, P154

Asma O. P153, P154

Asma Z. P155

Attia M. P97

Ayeb J. P216

Ayed K. P127

Baaklini C. P133

Bachouch I. P132, P173, P174

Bachouch I. P200, P201

Badoum G. P146

Baili H. P216

Balloumi N. P103

Bamha H. P77, P78, P79, P121

Batahar S.A. P168, P169, P171

Bawe L. P214

Be Saad S. P106, P107

Bechir M.B. P108

Belala R. P88

Belghol R. P177

Belhadj S. P106, P107

Belkhir D. P172

Belkhir S. P104, P105, P108, P131

Belloumi N. P132, P173, P174, P200, P201

Ben Ali M. P136, P137, P194, P195

Ben Amar J. P82, P83, P130, P131, P188, P189

Ben Amara K. P103
Ben Khamsa Jameleddine S. P127

Ben Mansour A. P106, P107, P206, P207

Ben Rhouma C. P205

Ben Saad A. P204, P205

Ben Saad S. P206, P207

Ben Salah N. P172, P178

Benaziz H. P170, P171, P199

Ben Jemia E. P82, P83, P130, P131, P151, P152, P179, P188, P189

Benjelloun H. P73, P75, P76, P89, P90, P98, P99, P100

Benjelloun M.C. P74, P156, P211

Benjelloune H. P95

Benzarti M. P91, P92, P93, P94, P116, P117

Benzarti W. P91, P92, P93, P94, P116, P117

Besma H. P108, P134

Bhairis M. P163

Biaou D.G. P214

Biaz Z. P74

Blibech H. P172, P178

Boguikouma J.B. P124, P125

Boncoungou K. P145, P146

Bouacida I. P103, P193

Bouafia S. P91, P92, P116, P117, P136, P137

Bouassida I. P209

Boubaker J. P105

Boubaker N. P104, P108

Boubaker N. P181

Bouchareb S. P216

Bouchnak I. P216

Bougacha M. P126, P167

Bougadoum M. P192

Bougma G. P145

Bougrini Y. P74, P177

Bougteb N. P109, P120

Boulingui C.M. P124, P125

Boumehdi A. P80

Bounoua F. P159, P160, P161, P162

Boussehra A. P76

Boussoffara L. P216

Brahem Y. P216

Chaabane M. P191

Chaâbi K. P96, P97

Chaabouni A. P150
Charfi M.R. P126, P167, P176, P213

Charpin D. P87

Chebbi R. P216

Cheikh Rouhou S. P118, P118, P191

Cheikhm'hamed S. P204, P205

Cherif H. P126, P167, P176

Cherkaoui R. P81, P84, P85

Chermiti F. P132, P173, P174, P200, P201

Chouchane H. P213

Christian J. P145

Christine E.M. P70

Cojocaru C. P86

Cojocaru E. P86

Dabboussi S. P181, P182, P183

Daghfous H. P106, P107, P206, P207

Daghfous J. P178

Debbiche S. P96, P97, P126, P176

Dhahri B. P118, P119

Dhahri B. P191, P215

Dingboe S. P71

Djebbar A. P144

Djeghr A. P88

Domagala-Kulawik J. P128

EEP Index

Efalou P. P214

El Gharbi L.D. P96, P97, P127

El Ghoul J. P184, P185, P186, P187

EL Hamdaoui L. P101, P102

EL Khattabi W. P101, P102

El kihal H. P106, P111

El Oueriachi F. P163

El Wadhane I. P178

Elbiaze M. P156

Elfidha S. P208

Elfkih W. P204

Elhamdaoui L. P79, P85

Elkhattabi W. P69, P77, P78, P79, P80, P81, P84, P85, P106, P109, P110, P111, P112, P113, P114, P115, P120, P121, P122

Elouazzani H. P164, P165, P166

Essabahi B. P211

Es-sabbahi B. P156 
Essebaa S. P206

Essid R. P209

Euchi K. P82, P83, P130

Fahem N. P204, P205

Fahmi A. P79

Farissi C. P89, P90

Fatma zahra D. P155

Feki W. P149, P150

Fenina W. P172, P178

Fenniche S. P132, P173, P174

Fenniche S. P200, P201

Ferchichi M. P104, P105, P106, P107

Gargouri I. P91, P92, P93, P94, P116, P117

Gargouri R. P149

Garrouche A. P91, P92, P93, P94, P116, P117

Ghanmi I. P96, P97

Gharbi Douik Leila El. P155

Ghrairi H. P136, P137

Gorgi Y. P189

Guediri N. P206

Habbouria C. P132, P173, P174, P200, P201

Hachicha A. P213

Hajjej S. P91, P92, P93, P94, P116, P117

Halloumi O. P202

Halwani C. P213

Hamdi B. P104, P105, P208

Hamoud S. P144

Hamza Z. P194, P195

Hamzaoui A. P104, P105

Hamzaoui A. P208

Handous R. P93, P94

Harmouche C. P133

Harraz H. P95

Hassane Kabiri Chirurgie Thoracique El. P163

Hassène B. P134, P175

Hayouni A. P91, P92, P93, P94, P116, P117

Hedhli A. P118, P119

Hedhli A. P191

Hedia G. P194, P195, P196

Hema A. P145

Hemissi K. P82, P83

Henda N. P138

Houcine Y. P103, P209

Iba Ba J. P124

Ibinda L.D. P124, P125

Ibn Hadj Amor H. P216

Ijim M. P139, P140, P142

Ikbel K. P108, P134

Imen C. P194, P195, P196
Imen G. P153, P154

Imen S. P155

Imen T. P175

Imzil A. P147, P148

Ines B. P138

Ines M. P155

Intissar C. P184, P185, P187

Iqraouin S. P156

Jabri H. P69, P77, P78, P79, P80, P81, P84, P85, P101, P102, P106, P109, P110, P111, P112, P113, P114, P115, P120, P121, P122

Jalel K. P175

Jalloul W. P98, P99

Jamel A. P108

Jankowska A.K. P129

Jarrar A. P151, P152

Jebali A. P178

Jebali R. P206, P207, P208

jihène A. P175

Joober S. P204, P205

Kaabi L. P208

Kabore F. P145

Kacem M. P118

Kaddoussi R. P204, P205

Kalboussi S. P136, P137

Kallel N. P150

Kamel K. P184, P185, P187

Kammoun S. P149, P150

Karaborni B. P126, P167, P176

Kechnaoui S. P190, P212

Keskes Boudawara N. P216

Khadhraoui M. P181, P182, P183

Khaled B. P196

Khalfallah I. P104, P105, P208

Khannous A. P159, P160, P162

Khayat G. P133

Khezami N. P132, P200, P201

Khezami N.H. P173, P174

Khlas E. P193

Klila M. P191

Knani J. P216

Knaz A. P91, P92, P116, P117

Kombila U.D. P124, P125

Kotti A. P149, P150

Koumeka P.P. P203

Kraiem S. P216

Ksissi S. P216

Kunakey E.K. P146

Kwiecien I. P128

L'her P. P87

Laklaai Z. P100

Langrand C. P129

Laouini I. P179

Leila B. P175
Limem I.B. P96, P97

Line K. P134

Lotfi S. P202

Louhaichi S. P104, P105

Louhaichi S. P208

Loukil M. P136, P137

Loukil M. P137

Louzir B. P172, P178

MA J. P172

Maalej S. P96

Maâlej S. P97

Magroun I. P206, P207

Manel L. P194, P195, P196

Marghli A. P103

Marghli A. P193

Marrakchi R. P150

Marzouki S. P208

Massongo M. P70

Mehiri N. P172, P178

Mejri I. P181, P182, P183

Merai S. P118, P119, P191, P215

Merghli A. P209

Meriam F. P108

Meridj A. P88, P138

Mhamdi S. P181, P182, P183

Migaou A. P204, P205

Minougou C.J. P145

Mjid M. P118, P119

Mjid M. P191, P215

Mkaddem S. P97

Mlika M. P193

Moatemri Z. P181, P182, P183

Mokaddem S. P127

Monia A. P138

Mosbah M. P83

Moubachir H. P147, P148, P159, P160, P161, P162, P202

Mounguengui D. P124, P125

Moussa N. P150

Moussounda G.S. P168, P169

Mrassi H. P132, P173, P174, P182, P200, P201

Msaad S. P149, P150

Msika S. P122

Naaroura A. P83, P118, P130

Nada G. P194, P195, P196

Nadia B.K. P175

Naji-Amrani H. P177

Narjes A. P194, P195, P196

Naroura A. P188, P189

Neffati O. P106, P107

Neji H. P193

Nouira I. P132, P173, P174

Nouira I. P200, P201
Omrane A. P93, P94

Ouaalaya E. H. P87

Ouahchi Y. P118, P119, P191

Ouakil N. P171, P197, P198, P199

Ouarssani A. P177

Ouedraogo A.R. P145, P146

Ouedraogo G. P145, P146

Ouedraogo G.A. P145, P146

Ouedraogo M. P145, P146

Ouerghi S. P103

Ouerghi S. P193

Ouerghui S. P209

Ouertani H. P130, P131

Pefura-Yone E.W. P70

Rachid C. P157, P158

Rachid C. P203

Rafitoharison N.L.E. P135, P143

Raftani S. P109, P120, P121, P122

Raharimanana R. P87

Raharimanana R.N. P135, P143

Raherison-Semjen C. P87

Rajaoarifetra J. P87

Rakotoarisoa J-CA. P87

Rakotomizao J. P87, P143

Rakotomizao J.R. P135

Rakotondrabe I.D. P135, P143

Rakotoson J. P87

Rakotoson J.L. P135, P143

Ralison A. P87

Randriamanana D. P87

Randriamananjara C. P87

Randriananahirana Z.A. P143

Raniszewska A. P128

Ranivoson A.H. P135, P143

Rasamimanana G. P87

Rattal A. P75

Ravahatra K. P135, P143, P87

Ravalison B. P87

Rebasy L. P135, P143

Rejeb S. P131

Rejeb W. P82

Rhorfi I. P164, P165, P166

Rjimati M. P211

Rouetbi N. P204, P205

Rutkowska E. P128

Rym K. P138

Sabri H. P153, P154

Sabrine F. P134

Sabrine L. P108, P134

Safa M. P134

Sahnoun I. P96, P97, P127

Saidani A. P149, P150

Saidi I. P210

Salma K. P138

Sana A. P153, P154 
Saoussen H.-Z. P138

Sarra B.S. P155

Sassine D. P133

Selmani M.R. P144

Serhane H. P147, P148, P159, P160, P161, P162, P202

Serraj M. P211

Serraj M. P74, P156

Sfar I. P189

Siham I. P180

Slaoui O. P163

Slim K. P194, P195, P196

Slim L. P106, P107

Snene H. P172

Snène H. P178

Soja-Kukieła N. P129

Sokolowski R. P128

Sonia M. P155
Souhi H. P164, P165, P166

Souhir K. P175

Sourabie A. P145, P146

Tahar O. P188, P189

ThiNhinh V.U. P123

Tiaray H.M. P135, P143

Tiaray Harison M. P87

Tiendrebeogo A. P146

Tlili K. P88

Tognon Tchegnonsi F. P71

Touil I. P216

Toujani S. P118, P191, P215

Toujani, Boubaker N S. P119

Trabelsi H. P127

Trabelsi J. P149

Triki M. P126, P167, P176

Tritar F. P106, P107, P206, P207

Turki S. P213
VanGiap V.U. P123

Virginie P.-M. P70

Wadhane I.E.L. P205

Wadhani I. P172

Wafa B. P153, P154

Wafa M. P184, P185, P186, P187

Warda J. P215

Wouoliyou N.A. P70

Yacine O. P215

Yangui F. P126, P167, P176, P213

Yangui F. P213

Yassine N. P175

Yassine N. P89, P90, P73, P75, P76, P95, P98, P99, $\mathrm{P} 100$

Yosra B. P175

Youakim C. P133
Zadi M. P77

Zaghba N. P73, P75, P76, P89, P90, P95, P100

Zaghba N. P98, P99

Zaibi H. P188, P189

Zaibi H. P82, P83, P130, P131, P151, P152, P179

Zairi S. P103, P193

Zayen K. P178

Zegmout A. P164, P165, P166

Ziane F. P72, P88

Zitouni A. P72

Zitouni Y. P88

Znegui T. P183

Zouiten S. P74, P211

Zribi H. P103, P193, P209 